\title{
WestVirginiaUniversity
}

THE RESEARCH REPOSITORY @ WVU

Graduate Theses, Dissertations, and Problem Reports

2009

\section{Transient flow characteristics of a high speed rotary valve}

Patrick H. Browning

West Virginia University

Follow this and additional works at: https://researchrepository.wvu.edu/etd

\section{Recommended Citation}

Browning, Patrick H., "Transient flow characteristics of a high speed rotary valve" (2009). Graduate Theses, Dissertations, and Problem Reports. 2894.

https://researchrepository.wvu.edu/etd/2894

This Dissertation is protected by copyright and/or related rights. It has been brought to you by the The Research Repository @ WVU with permission from the rights-holder(s). You are free to use this Dissertation in any way that is permitted by the copyright and related rights legislation that applies to your use. For other uses you must obtain permission from the rights-holder(s) directly, unless additional rights are indicated by a Creative Commons license in the record and/ or on the work itself. This Dissertation has been accepted for inclusion in WVU Graduate Theses, Dissertations, and Problem Reports collection by an authorized administrator of The Research Repository @ WVU.

For more information, please contact researchrepository@mail.wvu.edu. 


\title{
Transient Flow Characteristics of a High Speed Rotary Valve
}

\author{
Patrick H. Browning
}

\author{
Dissertation submitted to the \\ College of Engineering and Mineral Resources \\ at West Virginia University \\ in partial fulfillment of the requirements \\ for the degree of
}

\author{
Doctor of Philosophy \\ in \\ Aerospace Engineering \\ John L. Loth, Ph.D., Chair \\ Gary J. Morris, Ph.D. \\ Nigel N. Clark, Ph.D. \\ Scott Wayne, Ph.D. \\ George Richards, Ph.D.
}

Department of Mechanical and Aerospace Engineering

\author{
Morgantown, West Virginia \\ 2009
}

Keywords: Rotary Valve, Shock Tube, Transient Flow, Homogeneous Charge

Compression Ignition, Controlled Autoignition 


\section{ABSTRACT \\ Transient Flow Characteristics of a High Speed Rotary Valve}

Patrick H. Browning

Pressing economic and environmental concerns related to the performance of fossil fuel burning internal combustion engines have revitalized research in more efficient, cleaner burning combustion methods such as homogeneous charge compression ignition (HCCI). Although many variations of such engines now exist, several limiting factors have restrained the full potential of HCCI. A new method patented by West Virginia University (WVU) called Compression Ignition by Air Injection (CIBAI) may help broaden the range of effective HCCI operation. The CIBAI process is ideally facilitated by operating two synchronized piston-cylinders mounted head-to-head with one of the cylinders filled with a homogeneous mixture of air and fuel and the other cylinder filled with air. A specialized valve called the cylinder connecting valve (CCV) separates the two cylinders, opens just before reaching top dead center (TDC), and allows the injection air into the charge to achieve autoignition. The CCV remains open during the entire power stroke such that upon ignition the rapid pressure rise in the charge cylinder forces mass flow back through the CCV into the air-only cylinder. The limited mass transfer between the cylinders through the CCV limits the theoretical auto ignition timing capabilities and thermal efficiency of the CIBAI cycle. Research has been performed to:

1) Experimentally measure the transient behavior of a potential $\mathrm{CCV}$ design during valve opening between two chambers maintained at constant pressure and again at constant volume;

2) Develop a modified theoretical CCV mass flow model based upon the measured cold flow valve performance that is capable of predicting the operating conditions required for successful mixture autoignition;

3) Make recommendations for future CCV designs to maximize CIBAI combustion range.

Results indicate that the modified-ball CCV design offers suitable transient flow qualities required for application to the CIBAI concept. Mass injection events were experimentally mapped as a function of valve speed, inter-cylinder pressure ratios and volume ratios and the results were compared to compressible flow theoretical models. Specifically, the transient behavior suggested a short-lived loss-mode initiation closely resembled by shock tube theory followed by a quasi-steady flow regime resembling choked flow behavior. An empirical model was then employed to determine the useful range of the CCV design as applied to a four-stroke CIBAI engine cycle modeled using a 1-D quasi-steady numerical method, with particular emphasis on the cyclic timing of the $\mathrm{CCV}$ opening. Finally, a brief discussion of a high-temperature version of the CCV design is presented. 


\section{Dedication}

This work is dedicated to my mother,

\section{Mary Ann Kitzmiller Hughes, Ph.D.}

January 4, 1949 - February 26, 2005

I recall a time - I must have been about 13 years old then - when I sat and watched her as she "did research". Carefully, she marked and numbered each of her milkweed bugs with tiny specks of white paint followed by even tinier black handwritten numbers. She watched and recorded the behavior of those bug families in the same honest and meticulous way for years as part of her doctoral research which had been delayed and set aside for almost a decade for the sake of her young family. How little she knew the profound impact that would have on my own research habits so many years later!

My mother sacrificed much for all of us, but in the foolishness of my youth and the ado of my young adulthood I never got around to telling her how much I appreciated her. The next time I see her she will be healthy and happy, and I will not delay in telling her just how much she means to me. 


\section{Acknowledgements}

In research like this there is of course no such thing as a successful one-man effort. Several folks on many different levels have been directly or indirectly involved in seeing this work through to fruition.

I am especially grateful to my advisor and committee chair, Dr. John L. Loth, for his guidance and support throughout my entire graduate experience. In many ways his unique brand of practical-yet-theory-hardened methodology has had quite an impact on shaping my own approach to just about any engineering problem. I know his mentoring will continue to enrich my work for years to come.

I would like thank committee member Dr. Gary Morris, in particular for his expert guidance in the finer details of some of the theoretical assumptions I have had to make along the way, and also for his support in some of the trickier electronic problems I encountered during testing.

Many thanks are due to committee members Dr. Scott Wayne, Dr. Nigel Clark, and Dr. George Richards for their guidance in the combustion related areas of this research. I would also like to thank Dr. Jacky Prucz for providing his time during the last few stages of this doctoral work.

A huge amount of this work was spent in the MAE machine shop, and it is imperative that I acknowledge all of the time and mentoring offered to me by Clifford Judy. Cliff's natural machinist skill, his lifetime of experience, and his ability to "think outside the box" were absolutely critical in achieving a working test apparatus to perform this research.

Debbie Willis and Linda Cox will be names I will not likely forget for some time. I believe that without these two wonderful coordinators in the MAE offices, many graduate students would simply throw in the towel and give up. Without Debbie and Linda's expertise, I would not have been able to maneuver the maze of paperwork involved in "simply" graduating.

I must acknowledge the help and encouragement of several fellow graduate students that I have come to know over the years. My thanks to former doctoral student Richard Guiler for many long and fruitful academic discussions over the years, former doctoral student James Snider, II for his early recommendations and "grad student survival" insights, former doctoral student Fernando Echavarria for allowing me to work so closely with him on his Ph.D. research, and current masters student Shanti Hamburg and current doctoral student Paul Kreitzer for their interest and helpful insights into my research.

I am most aware of the integral part that my own family has played in helping me to reach this milestone in my life. I have had the blessing of a large and loving family, a fact that I have never taken lightly. I am grateful to my sons, Kane and Robbie, and my oldest daughter, Molly, for helping me read and record enormous logs of data that had to be transferred from original hand written format in test journals to electronic format in spreadsheets. Although their enthusiasm was perhaps at an all-time low sitting there reading off numbers, I'm proud of the fact that they stuck with it until the job was done. I suppose I owe some thanks to my youngest daughter, Annabelle, who frequently forced me to stop what I was doing at the computer so that we could take a chocolate milk 
break. I must express my eternal thanks to my lovely wife, Paula, for her enduring support no matter what I get myself into. Her support during these long school years has been more of a necessity than she knows, and I don't know how I would make it through this life without her by my side.

Finally, I must acknowledge my Creator in everything I do. Mankind is no random occurrence in God's great universe, and this is not the only life He has intended for us. It is by God's will alone that I draw every breath, it is by His grace that I am saved, and to Him should all praise be made.

I will praise thee; for I am fearfully and wonderfully made: marvelous are thy works; and that my soul knoweth right well. - Psalm 139:14

This is a faithful saying, and worthy of all acceptation, that Christ Jesus came into the world to save sinners; of whom I am chief. - 1 Timothy 1:15 


\section{Contents}

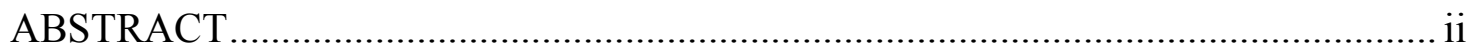

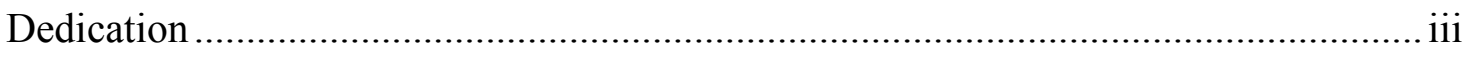

Acknowledgements ................................................................................................ iv

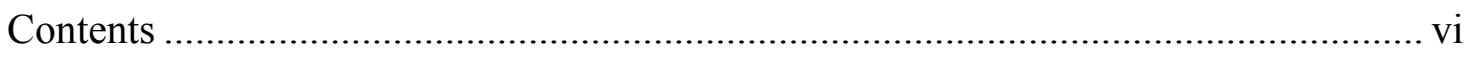

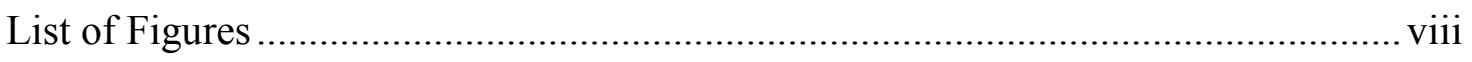

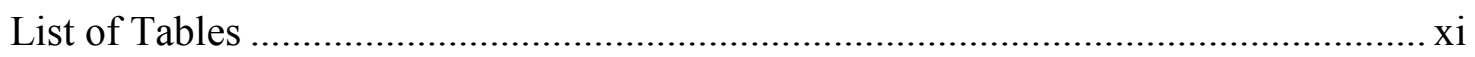

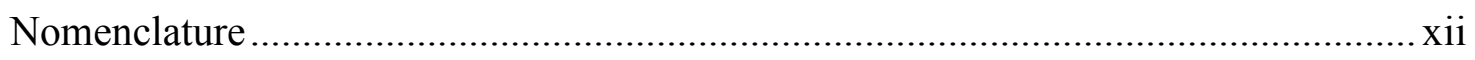

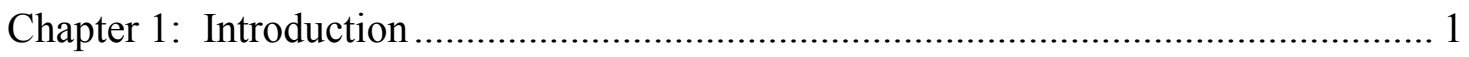

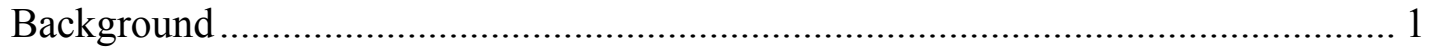

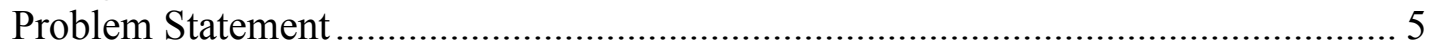

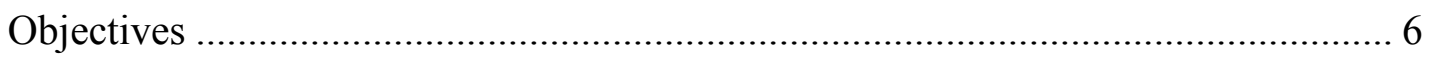

Chapter 2: Review of Relevant Literature …………............................................... 8 Homogeneous Charge Compression Ignition (HCCI) and Controlled Autoignition

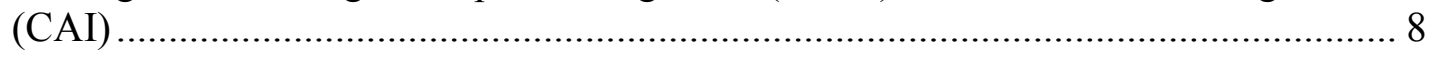

Compression Ignition by Air Injection (CIBAI) ................................................... 16

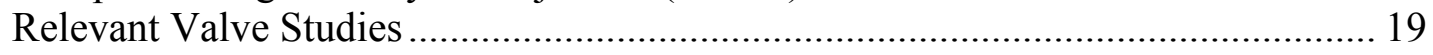

Chapter 3: CIBAI Cold Air Standard Analysis.............................................................. 24

Chapter 4: Non-Steady Valve Flow Modeling …………........................................... 36

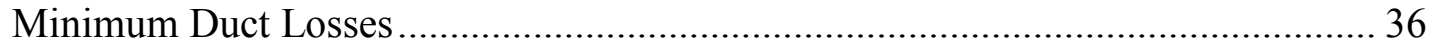

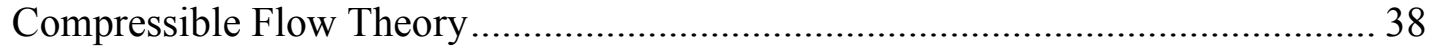

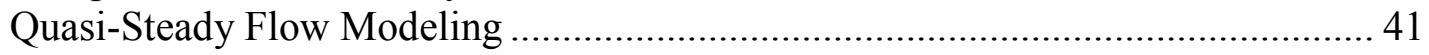

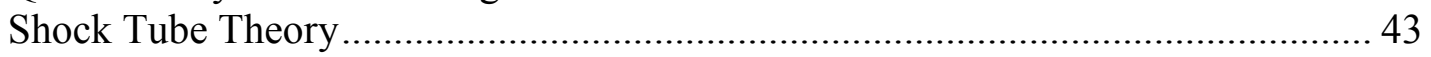

Chapter 5: Experimental Apparatus and Procedure ………………………............. 59

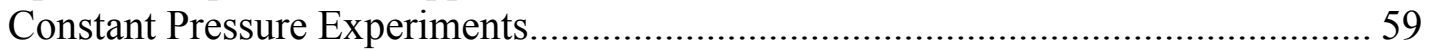

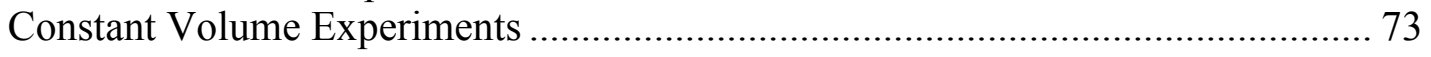

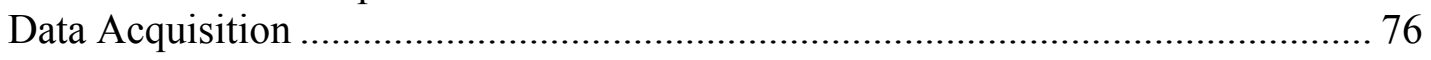

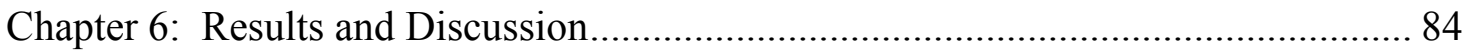

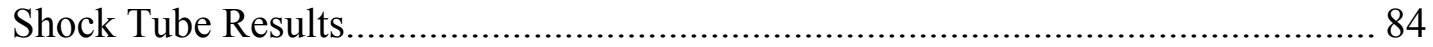

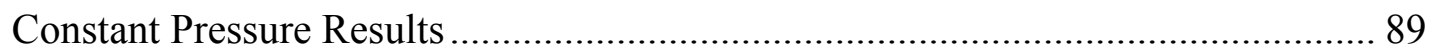

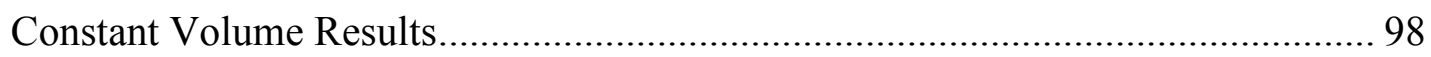

Quasi-Steady 1-D CIBAI Simulation Code Results ............................................... 102 


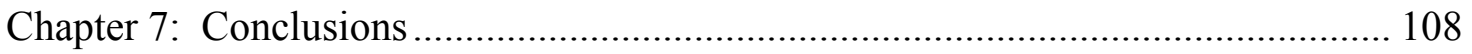

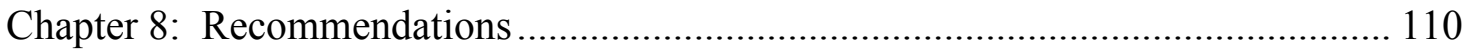

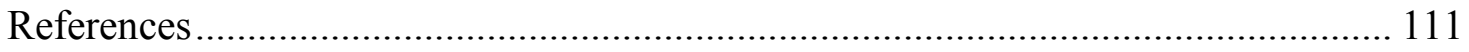

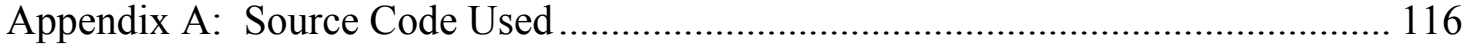

Quasi-Steady Main Program and Associated Subroutines .................................... 116

Omega DAQP-208H C++ Data Acquisition Control Code.................................. 124

Appendix B: Experimental Sensor Calibration................................................... 131

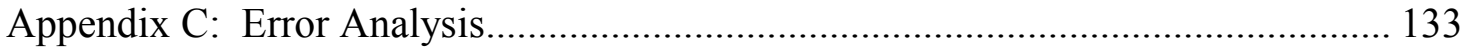

Error in calculated injected mass: ................................................................ 133

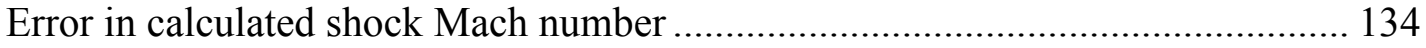

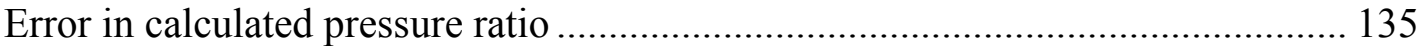

Error in CCV shaft speed calculations ........................................................... 136

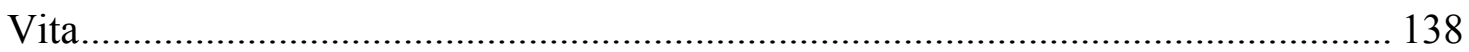




\section{List of Figures}

Figure 1: A four stroke CIBAI-based engine scheme, shown here with the high CR cylinder (left) connected beside the low CR cylinder (right) which allows

for single crankshaft power output (US Patent 6,994,057).....

Figure 2: A two stroke CIBAI based engine scheme served as the layout for early 1-D quasi-steady numerical studies- note the CCV, shown generically a simplified inter-cylinder block, is presently modeled using a theoretical discharge coefficient.

Figure 3: Early quasi-steady results of stable cycle-to-cycle combustion in a CIBAIbased 2-stroke engine configuration.

Figure 4: The air injection system used by Echavarria simulated actual CCV operation.

Figure 5: The wedge flow meter used in experimental correlations by Yoon et al.........20

Figure 6: The ideal cold air standard CIBAI cycle, shown producing net work out in $\mathrm{P}-\mathrm{v}$ and $\mathrm{T}$-s diagrams.

Figure 7: Thermal efficiency comparison of the CIBAI, Otto, and Diesel cycles operating under the following conditions: $\mathrm{CR}_{\mathrm{af}}=8.5, \mathrm{X}=5, \mathrm{VR}=11, \mathrm{k}$ $=1.4,8<\mathrm{CR}_{\mathrm{a}}<28$

Figure 8: Comparison of dimensionless MEP for CIBAI, Otto, and Diesel cycles for conditions: $\mathrm{CR}_{\mathrm{af}}=8.5, \mathrm{X}=5, \mathrm{VR}=11, \mathrm{k}=1.4,8<\mathrm{CR}_{\mathrm{a}}<28$.

Figure 9: Compressible flow equations can be applied to the simplified conditions above in which region 3 represents the fully open $\mathrm{CCV}$.

Figure 10: A shock tube wave system on the $(\mathrm{x}, \mathrm{t})$ plane (figure reproduced from [35])....

Figure 11: The shock tube at initial time, $t_{0}$ shows the high pressure region 4 separated from the low pressure region 1 by a thin diaphragm (D).

Figure 12: A starting shock wave $\left(\mathrm{S}_{1}\right)$, contact front $\left(\mathrm{C}_{1}\right)$, and rarefaction wave $\left(\mathrm{R}_{1}\right)$ immediately develop upon rupture of the diaphragm.

Figure 13: The first shock $\left(\mathrm{S}_{1}\right)$ is reflected at the end of the closed tube.

Figure 14: The first head-on collision in a tube of equal region 1 and region 4 initial lengths will be between the reflected shock and the contact front. ..............50

Figure 15: The head-on collision gives rise to a second rarefaction wave $\left(\mathrm{R}_{2}\right)$. ............52

Figure 16: The first rarefaction zone $\left(\mathrm{R}_{1}\right)$ is reflected at the end of the closed tube. .......53

Figure 17: The head-on collision of the reflected rarefaction wave $\left(\mathrm{R}_{3}\right)$ and the refracted shock wave $\left(\mathrm{S}_{3}\right)$.

Figure 18: The second rarefaction wave $\left(\mathrm{R}_{2}\right)$ reflection and the contact zone $\left(\mathrm{C}_{3}\right)$ created from the head-on collision of Figure 17.

Figure 19: A shock wave $\left(\mathrm{S}_{4}\right)$ is reflected from the end of the tube originally containing the high pressure gas.

Figure 20: The reflected shock $\left(\mathrm{S}_{5}\right)$ enters the spread out contact zone $\left(\mathrm{C}_{3}\right)$ head-on......56

Figure 21: Another rarefaction zone $\left(\mathrm{R}_{6}\right)$ is created as the refracted shock passes through the reformed contact zone $\left(\mathrm{C}_{4}\right)$ .56

Figure 22: After the reflection of the rarefaction wave $\left(\mathrm{R}_{7}\right)$, an older rarefaction zone $\left(\mathrm{R}_{4}\right)$ collides head-on with a contact front $\left(\mathrm{C}_{2}\right)$. 
Figure 23: Refraction $\left(\mathrm{R}_{9}\right)$ and reflection $\left(\mathrm{R}_{8}\right)$ of rarefaction zones after the collision of Figure 22.

Figure 24: The constant pressure experimental setup...............................................59

Figure 25: The 5 speed CCV motor was used to supply the constant RPM CCV shaft input.

Figure 26: The CCV assembly consisted mainly of an input clutch (1), input shaft adapter (2), CCV position sensor (3) and main ball housing (4).

Figure 27: The original ball (left) which had a straight 0.371 " hole was replaced with a new ball (right) which had a $90^{\circ} 0.375 "$ hole.

Figure 28: A cutaway view of the CCV design showing the inlet (1), modified exhaust location (2), CCV control stem (3), and pressure relief exhaust location (4).

Figure 29: Nozzles of various diameters were used to force the flow to choke at the exit of the CCV (diameters in thousandths of an inch are stamped on the nozzle bodies).

Figure 30: The shock tube assembly was capable of holding up to eight microphone sensors spaced along the tube wall in 1.500" increments (fluid moved from left to right as pictured).

Figure 31: The microphone sensors (1) required some associated hardware (2-5) to outfit the shock tube assembly.

Figure 32: An isometric view of the shock tube CAD model shown with 4 mounted microphone sensors on the top and the CCV assembly mounted at the inlet.

Figure 33: A closer cutaway view shows the CCV assembly, choke nozzle, and shock tube assembly with the first microphone station occupied and the second microphone station unused.

Figure 34: The injected mass collection chamber was made with a single inlet (1), a major vent (2), two minor vents (3), a precision steel rule (4) and an optional throttled chamber pressure tap (5).

Figure 35: A close up view of the mounted steel rule shows an initial chamber level of $139 / 64$ ".

Figure 36: The total injected mass of a single CCV turn was calculated based upon the geometry of the collection chamber and reservoir, and upon the initial and final heights of the chamber water level. .........................................70

Figure 37: The constant volume experimental setup. .73

Figure 38: A modified $\mathrm{CO}_{2}$ bottle was used as the downstream constant volume $(543 \mathrm{cc})$ tank in many of the constant volume tests.

Figure 39: A magnetic pickup switch (1) and a slotted IR beam splitter (2) mounted below the CCV motor shaft were used to indicate the motor speed and general position.

Figure 40: The fast response microphone used in the shock tube experiments..............79

Figure 41: The DAQ center was entirely contained within a 12 " x 10" x 5" aluminum box.

Figure 42: The Omega DAQP-208H PCMCIA card was used to collect experimental data. 
Figure 43: An example of a dual-microphone sensor response during an injection process reveals at least 5 distinct transient behavioral patterns.

Figure 44: A closer inspection of the microphone signals indicates the time lag $(\Delta \mathrm{t})$ between the first and second microphones' indications of sudden pressure rise.

Figure 45: The measured Mach number of the supposed starting shock wave indicated that the initial sudden pressure rise was actually caused by a Mach wave traveling sonically through the tube.

Figure 46: Injected mass from all test samples xx01 through xx05 ( $\left.\mathrm{p}_{\text {tank }} \approx 60 \mathrm{psig}\right) \ldots . . .90$

Figure 47: Injected mass from all test samples xx06 through $\mathrm{xx} 10\left(\mathrm{p}_{\mathrm{tank}} \approx 50 \mathrm{psig}\right) . \ldots . .91$

Figure 48: Injected mass from all test samples $\mathrm{xx} 11$ through $\mathrm{xx} 15\left(\mathrm{p}_{\mathrm{tank}} \approx 40 \mathrm{psig}\right) . \ldots . .91$

Figure 49: Injected mass from all test samples $x \times 16$ through $x \times 20\left(\mathrm{p}_{\text {tank }} \approx 30 \mathrm{psig}\right) \ldots . . .92$

Figure 50: Injected mass from all test samples xx21 through $\mathrm{xx} 25\left(\mathrm{p}_{\mathrm{tank}} \approx 20 \mathrm{psig}\right) . \ldots . .92$

Figure 51: Injected mass from all test samples $x \times 26$ through $\mathrm{xx30}\left(\mathrm{p}_{\mathrm{tank}} \approx 10 \mathrm{psig}\right)$......93

Figure 52: Injected mass from all test samples $\times 201$ through $\times 230(\mathrm{RPM} \approx 1075) \ldots \ldots . .93$

Figure 53: Injected mass from all test samples $\times 301$ through $\times 330(\mathrm{RPM} \approx 995) \ldots \ldots \ldots . .94$

Figure 54: Injected mass from all test samples $x 401$ through $x 430(\mathrm{RPM} \approx 975) \ldots \ldots \ldots . .94$

Figure 55: Injected mass from all test samples $\times 501$ through $\times 530(\mathrm{RPM} \approx 940) \ldots \ldots \ldots . .95$

Figure 56: Injected mass from all test samples x601 through x630 $(\mathrm{RPM} \approx 860) \ldots \ldots \ldots . .95$

Figure 57: The modified injection mass pi term was highly correlated to the inter-

CCV pressure ratio

Figure 58: High frequency pressure transducer data was the primary source of experimental data during the constant volume experiments.

Figure 59: Experimental, theoretical, and empirical dimensionless results demonstrate the capability of the method to predict CCV injection behavior.

Figure 60: Lower values of total pressure ratios were equally well suited for the empirical equation because the form still used the choked flow notion of minimum area, $\mathrm{A}^{*}$

Figure 61: The fixed geometric behavior of the CCV, intake, and exhaust valves over 3 full cycles.

Figure 62: The pressure trends over 3 full cycles (6600 RPM) ..................................104

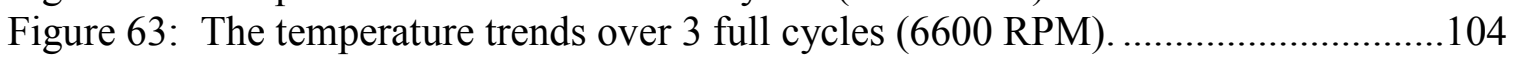

Figure 64: The mass trends over 3 full cycles (6600 RPM) .................................... 105

Figure 65: The energy trends over 3 full cycles (6600 RPM)..................................105

Figure 66: Potential autoignition for the paired set of $30 \mathrm{cc}$ engines in a CIBAI configuration.

Figure 67: Potential autoignition for the paired set of $70 \mathrm{cc}$ engines in a CIBAI configuration.

Figure 68: The calibrated curve of the PX-105 pressure transducer used in the second stage tank.

Figure 69: The calibration curve of the tank thermocouple. 


\section{List of Tables}

Table 1: Electronic sensors were used in a variety of locations to collect the relevant

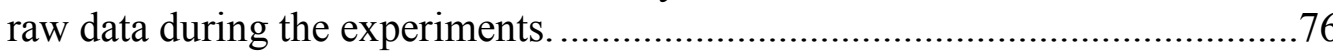

Table 2: Six different input channel arrangements were used during different stages of experiments to maximize the allowable scanning frequency.........................82

Table 3: The distinct transient behavioral patterns are easily categorized according to the instantaneous CCV behavior and the corresponding dominant stimulus. ...86

Table 4: A test matrix was used for each test series " $x$ " in which the mass injection behavior as a function of CCV speed and pressure ratios was systematically investigated.

Table 5: A test matrix was used for each constant volume test series " $\mathrm{xx}$ " in which instantaneous mass flow rate as a function of CCV speed, initial pressure ratios, and volume ratios was systematically investigated

Table 6: Test results for a quasi-steady engine operating with fixed gear CCV may be capable of a tight range of potential auto ignition times 


\section{Nomenclature}

\section{Abbreviations}

AEP

AFR

AIP

ATDC, BTDC

BDC

BMEP

CAD

CAI

CA50

$\mathrm{CCV}$

CIBAI

CFR

CI

$\mathrm{CO}$

CR

E

EGR

EVC

EVO

HCCI

iEGR
Exhaust port area

Air/fuel ratio

Intake port area

After top dead center, before top dead center

Bottom dead center

Brake mean effective pressure

Crank angle degrees

Controlled autoignition

Crank angle degrees for $50 \%$ fuel heat release

Cylinder connecting valve

Compression ignition by air injection

Cooperative Fuel Research

Compression ignition

Carbon monoxide

Compression ratio

Energy ratio

Exhaust gas recirculation

Exhaust valve closing

Exhaust valve opening

Homogeneous charge compression ignition

Internal exhaust gas recirculation (hot gas trapping) 
I

IC

IVC

IVO

MEP, IMEP

MON

MPRR

$\mathrm{NO}_{\mathrm{x}}$

NVO

OI

$\mathrm{ON}$

PM

PRF

RON

RPM

SI

SOC

SOI

TDC

VVA

VVT

WVU
Integral of $\mathrm{CCV}$ open area with respect to shaft angle

Internal combustion

Intake valve closing

Intake valve opening

Mean effective pressure, indicated MEP

Motor octane number

Maximum pressure release rate

Oxides of nitrogen

Negative valve overlap

Octane index

Octane number

Particulate matter

Primary reference fuel

Research octane number

Revolutions per minute

Spark ignition

Start of combustion (10\% burn)

Start of ignition

Top dead center

Variable valve actuation

Variable valve timing

West Virginia University 


\section{Symbols}
A
Area
$\mathrm{C}_{\mathrm{d}}$
Flow discharge coefficient
$\mathrm{D}_{\mathrm{h}}$
Hydraulic diameter
$f$
Friction factor, quasi-steady discharge coefficient factor
$\mathrm{k}$
Specific heat ratio
M
Mach number
$p$
Local pressure (absolute)
$\mathrm{P}$
Perimeter
$\mathrm{R}_{\mathrm{V}}$
Top dead center air/fuel-to-air cylinder volume ratio
$\mathrm{R}_{\alpha}$
Gas constant, species $\alpha$
$\mathrm{Re}_{\mathrm{x}}$
Reynolds number, with respect to generic length quantity $\mathrm{x}$
$\mathrm{T}$
Local temperature (absolute)
VR
Bottom dead center air-to-air/fuel cylinder volume ratio
V
$\alpha_{50}$
$\gamma$
$\rho$
$\eta$
$\lambda$
$\phi$
$\mu$
Volume
Angular location of $50 \%$ heat release
Specific heat ratio
Local density
Efficiency
Air/fuel ratio
Fuel/air equivalence ratio
Dynamic viscosity 


\section{Subscripts}

$\begin{array}{ll}\text { af } & \text { Air-only } \\ \text { avg } & \text { Air-fuel } \\ f & \text { Average value } \\ i & \text { Final value } \\ \text { max } & \text { Initial value } \\ \text { min } & \text { Maximum value } \\ \text { w, wall } & \text { Minimum value } \\ 0 & \text { Wall value } \\ & \text { Reference/total (stagnation) value }\end{array}$

\section{Notations}
$\Delta$
Difference
$\dot{x}$
Time rate of change of generic quantity $\mathrm{x}$ 


\section{Chapter 1: Introduction}

\section{Background}

Recent developments in the condition of the global liquid fossil fuel economy, namely the increase in demand coupled with the projected decline in production, has brought a renewed interest in more efficient internal combustion (IC) engine concepts. At the same time, increasing awareness of the negative ecological impact of combustion byproducts including $\mathrm{NO}_{\mathrm{x}}$ and particulate matter (PM) has driven a need for cleaner IC engine methods. The homogeneous charge compression ignition (HCCI) engine has demonstrated efficiency advantages over the modern spark ignition (SI) engine and emissions advantages over the modern compression ignition (CI) engine and represents a "best of both worlds" alternative to traditional IC cycles. Consequently, the realization of a robust commercially viable engine has been a major goal in recent research $[8,9,10,34]$. The emphasis of current research has focused on the methods of controlled autoignition (CAI), which directly address the need for rapid and appropriate engine parameter modification to enable optimum HCCI operation over a range of engine loads and speeds.

Loth and Morris at West Virginia University have patented a new cycle called "Compression Ignition by Air Injection", or CIBAI, to facilitate HCCI [6,7]. The novel approach of CIBAI in a reciprocating IC engine requires two piston cylinder assemblies to be mounted head-to-head or side-by-side (Figure 1), with a cylinder connecting valve (CCV) positioned in between the two. A homogeneous charge of air-fuel mixture is held in the relatively low compression ratio $(\mathrm{CR})(<8.5)$ cylinder, while a charge of air is held in the relatively high $\mathrm{CR}(>15)$ cylinder. The pistons of each cylinder operate in 
synchronization, and at a predetermined crank angle position near top dead center (TDC), the CCV opens allowing the high pressure/high energy air to accelerate into the air-fuel cylinder. If timed correctly, the introduction of mass and energy into the air-fuel cylinder can incite autoignition. The resulting expanding gas can then be harnessed as net work out via both pistons by allowing the CCV to remain open during the entire power stroke.

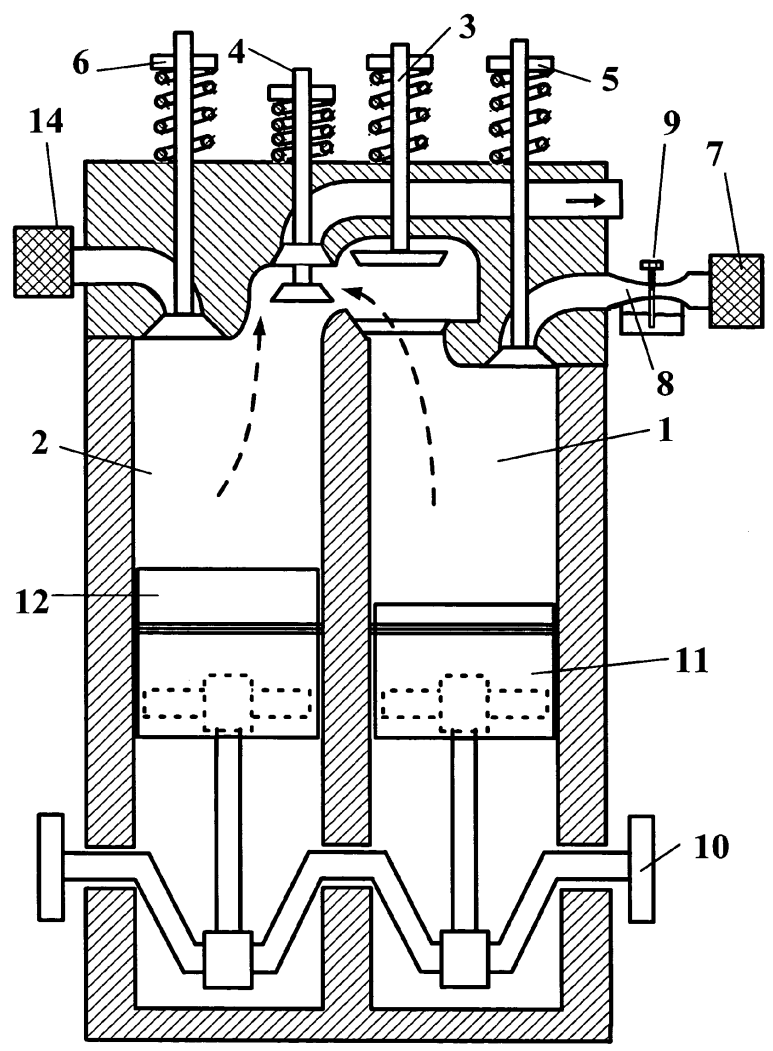

Figure 1: A four stroke CIBAI-based engine scheme, shown here with the high CR cylinder (left) connected beside the low CR cylinder (right) which allows for single crankshaft power output (US Patent 6,994,057).

The CIBAI cycle has been experimentally validated to some degree by work performed by Echavarria at WVU in 2006 on a variable compression ratio Cooperative Fuel Research (CFR) engine [1]. Based on the positive results of this earlier simulation work, further efforts toward the implementation of this new cycle were warranted. A 
traditional 1-D quasi-steady numerical model [2] was developed by Browning in an effort to establish dynamic trends of the CIBAI-based two stroke engine configuration shown in Figure 2. Major interest was placed on the theoretical requirements of CCV timing, in particular on the mass flow behavior under varying operating parameters. The results of some early code predictions based upon CCV discharge coefficient assumptions are presented in Figure 3. Although the temperature traces from both cylinders appear to demonstrate stable cycle-to-cycle combustion, the appropriate selection of precise injection initiation is at present found through a time consuming iterative process. In general, the quasi-steady code indicates high sensitivity of stable operation to input parameters such as air/fuel ratio, intake temperature, and CCV injection timing, too name just a few.

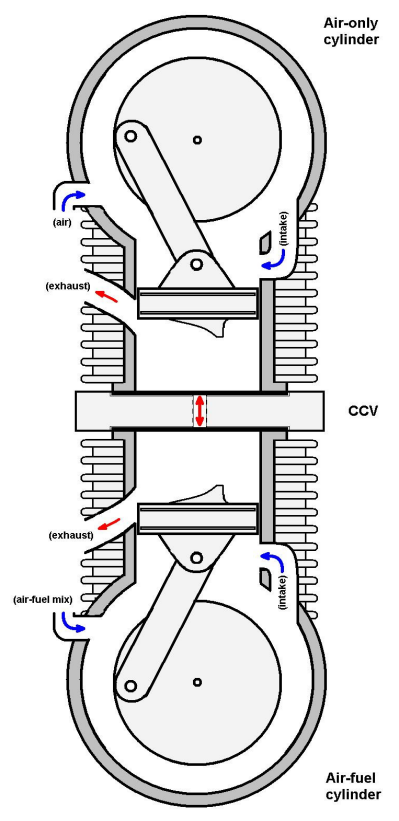

Figure 2: A two stroke CIBAI based engine scheme served as the layout for early 1-D quasi-steady numerical studies- note the $\mathrm{CCV}$, shown generically a simplified inter-cylinder block, is presently modeled using a theoretical discharge coefficient. 

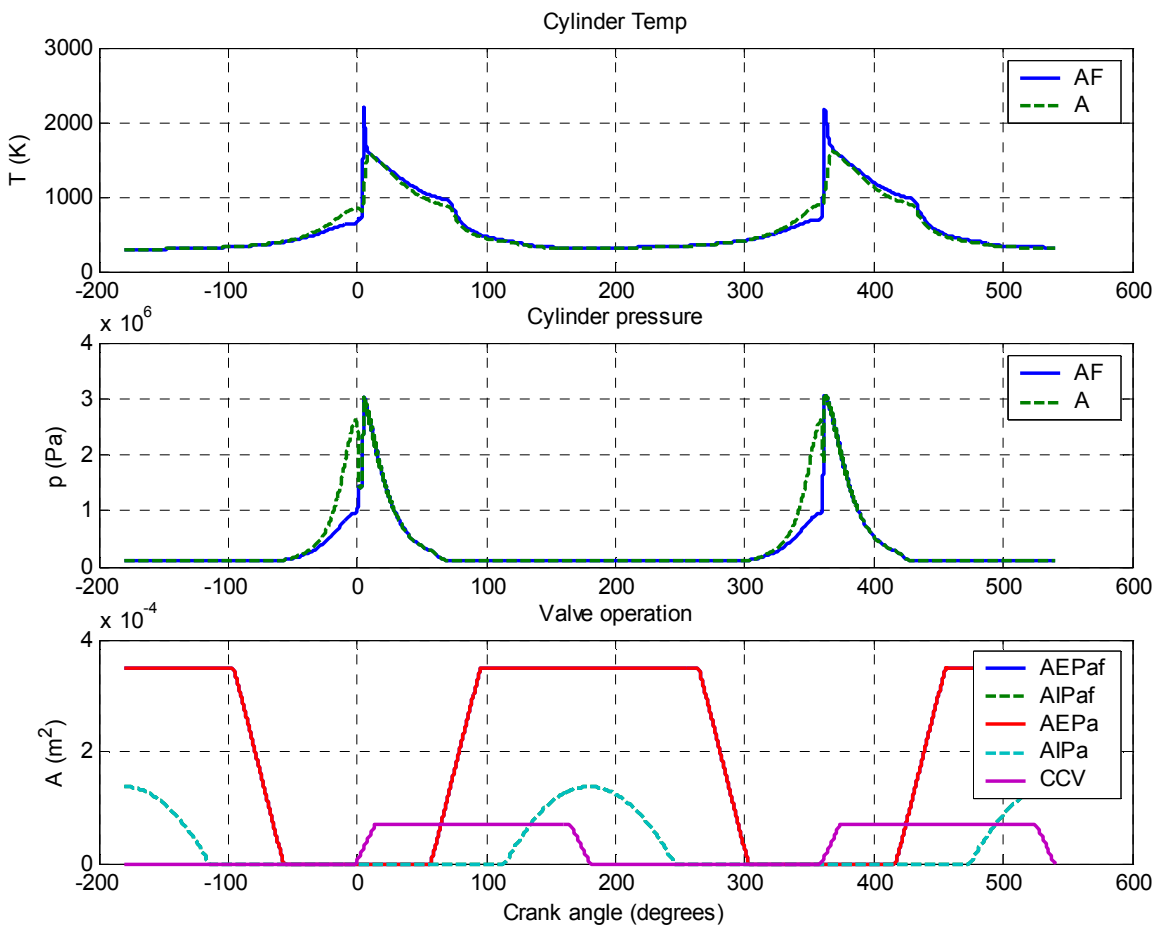

Figure 3: Early quasi-steady results of stable cycle-to-cycle combustion in a CIBAI-based 2-stroke engine configuration. 


\section{Problem Statement}

Based upon results of the 1-D quasi-steady model, it is evident that effective operation of the $\mathrm{CCV}$ is the key to enabling dependable cycle-to-cycle CIBAI operation. Successful configuration directly depends upon CCV related issues such as: appropriate crank angle valve initiation, valve discharge coefficient behavior over a range of operating speeds/loads, and to a lesser extent on valve power requirements. Although successful in demonstrating HCCI after a timed injection event, the earlier experimental valve simulation by Echavarria did not attempt to quantify the behavior of the valve in any way that would provide a basis for CCV fabrication [1]. It is clear that without knowledge of $\mathrm{CCV}$ limitations and design constraints, progress in the realization of a CIBAI-based HCCI reciprocating IC engine for additional research will be severely stunted. 


\section{Objectives}

The objective of the study was to clarify some of the unknown behavioral characteristics of at least one theoretically viable CCV. The work was broken into the following underlying objectives:

1) Design and fabricate at least one $C C V$. It needed to be capable of sealing reasonably well under a range of pressure differentials and rotational speeds, and be able to support at least one temperature sensor (thermocouple or thermistor) and a high response rate pressure transducer (piezoelectric or diaphragm). Power input to the CCV needed to be deliverable via a standard diameter shaft with high speed electronic torque disconnection and braking capabilities.

2) Experimentally determine $C C V$ behavior. The experiment needed to measure mass flow rate through the valve and attempt to correlate the calculated discharge coefficients to multiple governing dimensionless parameters including dimensionless pressure differential, upstream and downstream volume ratio, and dimensionless shaft speed. Two test sets were used to gather the data: first, a "transient open" system established the effect of shaft rotation on expected mass injected; second, a "transient closed" system established the additional effect of transient pressure changes in the upstream and downstream fixed volumes on total mass flow.

3) Modify 1-D numerical code CCV subroutine using experimentally determined trends. Correlated trends from step 2) were applied to the quasi-steady CIBAI 
model for the purpose of refining the modeled CCV discharge coefficient such that the potential for coefficient variation was considered. 


\section{Chapter 2: Review of Relevant Literature}

\section{Homogeneous Charge Compression Ignition (HCCI) and Controlled Autoignition (CAI)}

\section{Overview}

HCCI combustion has been of interest to researchers, particularly in the last two decades, as a potentially useful method of increasing IC engine efficiency while simultaneously reducing overall production of harmful combustion byproducts such as $\mathrm{NO}_{\mathrm{x}}$ [12]. It has been astutely pointed out in recent literature [11] that the favorite acronym "HCCl" is something of a misnomer in most cases. This is because the mixed charge in a practical sense is never fully homogeneous, as shown by Onishi et al. and Lavy et al. [11]. More recent research by $\mathrm{Xu}$ et al. has demonstrated the highly inhomogeneous combustion initiation in images taken during operation of an optical Jaguar 2.5L V6 HCCI engine [15]. Zhao et al. also point out that "compression ignition" is not truly representative of the combustion initiation. This statement is largely based on the demonstrated high sensitivity of the combustion event to intake temperature (heated fresh or hot exhaust gas recirculation) [17,11]. The more accurate acronym, especially for experimental engine designs, in describing the IC combustion most researchers and automotive manufacturers are striving for is "CAI". This statement is well founded in the basic premises that first to be useful the IC engine must be controllable in all operation ranges- hence "C", for "controlled"- and second the combustion event is completely described by the more enveloping term "autoignition"- hence "AI". Because the vast 
majority of literature refers to the more ideal case, the popular term "HCCI" will be used throughout the remainder of this work.

Many different methods have been devised to facilitate (control) repeatable HCCI operation. Every method, in one way or another, seeks to establish conditions favorable for autoignition at or near TDC for near-constant volume heat addition caused by the rapid heat release rate of the fuel oxidation. The most critical conditions pertaining to the autoignition process appear to be initial intake temperature and charge dilution [11]. The most critical aspect to any of the proposed methods is that it be capable of controlling the combustion timing from one cycle to the next. Proposed methods include:

- variable valve timing (VVT), or actuation (VVA), actively modifying the timing of the cylinder intake and/or exhaust valves, often employing negative valve overlap (NVO) $[14,15,16,9]$

- $\quad$ intake preheating and pressure boosting $[15,11]$

- fuel chemistry control $[12,33]$

The commercial research and development of HCCI engines and related support systems is remarkably active [34]. Dual mode HCCI-SI prototypes are appearing in General Motors' (GM) Opel Vectra and Saturn Aura in the form of the 2.2L 4-cylinder EcoTech engine. Diamler's new 1.8L 4-cylinder DiesOtto HCCI prototype engine has been demonstrated in the Mercedes F700 concept, and Volkswagen (VW) Group's Gasoline Compression Ignition (GCI) engine has advanced to the new 1.6L FSI model. All the prototypes claim as much as $15 \%$ increase in fuel efficiency, but each has a slightly different method of control. GM's EcoTech uses VVT and a "combustion management program"; Diamler's DiesOtto uses VVT, and variable compression ratio 
methods; VW's FSI uses VVA and "advanced engine management" $[8,9,10]$. The "management" programs utilized by GM and VW likely incorporate intake preheating and rapid fuel chemistry control methods to facilitate the quoted HCCI operating ranges of 25 to $60 \mathrm{mph}$ car speeds, outside which range the engines default back to SI mode [9].

\section{Range of Operation}

A large effort has been made by researchers to establish and eventually broaden the limits of useful HCCI combustion $[15,16,21,12]$. At present, the range of effective HCCI is bounded by both low and high engine speeds and low and high engine loads. The bound of "effective" HCCI may be determined from two distinct classes of constraints. These conditions are readily seen when the engine reaches a point at which an increase in engine performance (i.e., load or speed) would either no longer be controllable from one cycle to the next, or the performance would not yield the desirable blend of fuel efficiency and exhaust pollutant levels.

According to recent research by Yang at the Ford Motor Company [16], the limits of operation can be loosely defined by a number of phenomena. The upper load limit is typified by the appearance of "intolerable combustion roughness and knock." Xu et al. also detail the low-speed, high load limit as a function of knock and rough combustion [15]. An additional problem contributing to the high load bound is the increased production of $\mathrm{NO}_{\mathrm{x}}$, which rises sharply as a result of the reduced opportunity for mixture dilution and resultant boost in peak gas temperature, $T_{\max }$. In particular, this rapid increase occurs near $\mathrm{T}_{\max }>(1800-1900) \mathrm{K}$. Air induction can also be a constraint at high engine loads and speeds especially when negative valve overlap (NVO) strategies are used to control exhaust gas recirculation (EGR) and gas trapping (iEGR) $[15,16]$. Lower 
load limits are basically established at a point below which the engine is incapable of producing dependable cyclic work. The increase in cycle-to-cycle variation is largely due to the reduced thermal energy necessary for autoignition. This variation contributes to increased fuel consumption due to non ignition events, and high $\mathrm{CO}$ exhaust content owing to the incomplete conversion to $\mathrm{CO}_{2}$ from lack of $\mathrm{O}_{2}$ and reduced $\mathrm{T}_{\max }$.

\section{Heat Release and Transfer}

In an effort to better understand the HCCI process, the specifics of fuel heat release under varying conditions of engine operation must be quantified. Highly detailed numerical analysis can be performed using chemical kinetics modeling such as CHEMKIN $[15,13,20]$ (sometimes coupled with KIVA [17]), direct numeric simulation (DNS) [13], and the hydrodynamics, chemistry, and transport (HCT) model [18]. Experimentally, much emphasis has been placed on studying heat release behavior in terms of the position in crank angle degrees (CAD) of 50\% of fuel heat release, referred to in most literature as either CA50 or $\alpha_{50}[21,13]$. Experimental research by Kalghatgi and Head [21] indicate that the value range of CA50 tends to indicate the relative cycleto-cycle stability of an HCCI engine. The coefficient of variation (COV, defined as the ratio of standard deviation to mean value) of indicated mean effective pressure (IMEP) was used by the researchers as an indicator of cycle instability. They presented a formula based on CA50 and equivalence ratio, $\varphi$, to aid in determining the likelihood of stable operation such that,

$$
X=1.55 C A 50-52 \varphi .
$$

IMEP COV was found to be less than $10 \%$ (i.e., more stable HCCI operation) in $80 \%$ of the cases studied where $\mathrm{X}$ was a negative value. If, on the other hand, $\mathrm{X}$ was positive, the 
corresponding IMEP COV value was under $10 \%$ in only $12 \%$ of the cases (i.e., instability was much more likely) [21]. Their work also produced a simple method of predicting CA50 based upon fuel octane index (OI) and a handful of other engine operating conditions.

Heat transfer models within the cylinder are critical to numerically estimating conditions pertinent to autoignition. Several correlations exist, such as the many variations of Woschni's equation [2,20,13], for example,

$$
h_{c}=3.26 B^{-0.2} p^{0.8} T^{-0.55}\left(2.28 S_{p}\right)^{0.8}
$$

where $\mathrm{B}$ is piston bore and $\mathrm{S}_{\mathrm{p}}$ is the mean piston speed. The energy transfer rate out of the cylinder is given by

$$
\dot{Q}_{w}=h_{c} A_{w}\left(T-T_{w}\right)
$$

where $A_{w}$ and $T_{w}$ are wall area and temperature, respectively.

\section{Fuel Chemistry \& Mixture Modification}

Several studies have been performed to characterize the effects of fuel chemistry and in-cylinder mixture modification. Work performed by Smythe and Bryner established an enormous $\log$ of autoignition (AI) temperatures for a variety of hydrocarbon fuels exposed for short durations to several different types of heated metal foils [33]. Several interesting and perhaps useful pieces of information from their research include the following trends:

- AI temperature exhibits virtually no dependence on fuel-air mixture velocity, except for the case of nearly direct impingement normal to the heated surface at which point the AI temperature dropped by up to $100 \mathrm{~K}$; 
- n-octane fuels tended to show stable AI temperatures (within $40 \mathrm{~K}$ ) over a range of $\varphi$;

- in general, the presence of heated titanium surfaces showed the highest AI temperature while stainless steel (SS) showed the lowest.

Atkins and Koch [12] experimentally established a definitive collection of operating bounds and parameters using several different octane number $(\mathrm{ON})$ primary reference fuels (PRFs). In general, ON was found to retard the start of combustion (SOC, defined as the position in crank angle degrees, CAD, at which $10 \%$ of the fuel is burned) although the duration of burn remained largely independent of ON. Their research indicates that EGR and $\varphi$ are far more effective at manipulating SOC as compared to ON modification alone. However, EGR was ultimately found to be more effective at retarding SOC than $\varphi$. As in traditional SI engines, higher ON fuel was found to increase the high load limit of HCCI combustion owing to the fuel's increased resistance to autoignition [12]. Rather than attempt any curve fitting of the collected data, Atkins and Koch present the details of the experiments as useful air/fuel ratio $(\lambda)$ versus EGR maps with event contours in terms of CAD [12].

\section{EGR, Boosting, and Preheating}

Exhaust gas recirculation (EGR), as the name implies, utilizes the re-induction of a given percentage of the byproducts from previous combustion events to mix with the fresh air-fuel charge. This is achieved through either downstream valve mixing or cylinder valve timing control such that a portion of the hot exhaust gas remains trapped in the cylinder for use in the next cycle. This latter method is often referred to in literature as EGR trapping, iEGR, or residual or hot gas trapping $[11,15]$. Several current literature 
sources note that EGR and iEGR may be the most easily adjustable and effective way of expanding the limits of HCCI operation [17,11,15,19,14]. Zhao et al. [11] have suggested four basic effects of EGR:

- the charge "heating effect" describes the increase in intake temperature due to the addition of hot EGR with cool fresh air;

- the "dilution effect" describes the net reduction in oxygen/air as a result of EGR replacement of fresh intake air;

- the "thermal effect" describes the rise in heat capacity of the intake mixture due to the higher specific heats of the EGR-contributed water and carbon dioxide as opposed the fresh air it replaced;

- and the "chemical effect" describes the impact of certain combustion products on the autoignition process.

The results of their experiments indicated that autoignition combustion duration was almost purely a function of EGR- the greater the EGR, the longer the heat release took to complete. This increase in burn duration was determined to be equally caused by the dilution and thermal EGR effects. Although the thermal effect of trapped gas EGR, or iEGR, was determined to retard the start of ignition (SOI), the charge heating effect of iEGR had an SOI advancing effect of greater magnitude, thus the overall effect of iEGR was found to advance SOI. EGR thermal effect was the major factor in reducing the maximum pressure release rate (MPRR), but the chemical effect of EGR had a negligible impact on the MPRR (hence, no chemical effect on heat release rate). Finally, the experiments of Zhao et al. showed that an increase in NO formation due to an intake 
temperature increase could be reduced by increasing EGR. This was found to be a direct result of the dilution effect of the EGR [11].

Boosting, a process whereby the intake manifold pressure is raised above ambient pressure, is a common method used to combat the problems associated with poor breathing side effects of HCCI combustion at high speeds/loads. Xu et al. [15] have demonstrated that a supercharged air intake system coupled with an advanced exhaust valve closing (EVC) can effectively increase the HCCI high load limit. The authors also showed that advancing intake valve opening and closing (IVO and IVC, respectively) while increasing boost pressure and air/fuel ratio (AFR) can result in an increased HCCI load limit [15].

Intake temperature modification has been shown to have the most dramatic effect on control of autoignition timing. Jia and Xie showed that the angular location of 50\% heat release (CA50) retarded by as much as 25 crank angle degrees (CAD) for a $15 \mathrm{~K}$ temperature reduction in intake air [17]. Results by Yang showed that the dependence of CA50 was impacted highly by load, for example at higher loads (e.g., MEP $\approx 4.8$ bar), CA50 advanced $12 \mathrm{CAD}$ for a $10 \mathrm{~K}$ increase in intake temperature but at about half that load (MEP $\approx 2.8$ bar) CA50 advanced $14 \mathrm{CAD}$ for a $50 \mathrm{~K}$ increase in intake temperature [16]. Zhao et al. showed a similar trend when investigating SOC, demonstrating a retardation of SOI by about $20 \mathrm{CAD}$ for a $100 \mathrm{~K}$ reduction in intake temperature [11]. 


\section{Compression Ignition by Air Injection (CIBAI)}

\section{Patented Concept}

The concept of the CIBAI cycle originated at West Virginia University (WVU) in 2004. Two separate patents filed by WVU professors John Loth and Gary Morris [7,6] detail both two and four stroke versions of sample CIBAI-based engines.

In its most basic and ideal sense, the CIBAI thermodynamic cycle emulates a high compression ratio (CR) Otto cycle in that it exhibits constant volume heat addition that can rise to effective $\mathrm{CR}$ values beyond traditional Otto CR knock limits. This behavior can be realized in a reciprocating engine by compressing a premixed homogeneous charge without danger of knock $(\mathrm{CR}<\sim 10)$ in one piston-cylinder assembly. Operating in synchronization with the air-fuel cylinder, a separate high CR cylinder pressurizes a charge of air-only. Close to the point of maximum compression, at or just prior to top dead center (TDC), a cylinder connecting valve (CCV) actuates to allow the higher pressure air-only charge to transfer into the air-fuel cylinder. The sudden increase in pressure and temperature in the air-fuel cylinder incites autoignition of the mixture. Because the $\mathrm{CCV}$ is designed to remain open from the point of injection initiation to the following bottom dead center (BDC) position, the resultant rapid heat release and increase in pressure becomes a power stroke expansion utilized in both cylinders.

Based upon the general cycle description, the most recent patent claims six potential gains offered to modern IC engines including:

1) a general efficiency improvement due to the lack of a throttle (and associated throttle loss) and increased pressure during expansion; 
2) a wider range of acceptable fuels for cold starts due to increased ignition energy of the air injection event;

3) an increase in dependability and decrease in production cost due to the lack of high maintenance spark plugs (SI engines) or high pressure fuel injectors (CI engines);

4) a highly scalable design allowing miniaturization beyond current Diesel engines due to better fuel metering capability for the premixed charge;

5) a more thorough scavenging technique in CIBAI-based 2 stroke arrangements allowing cleaner operation than traditional 2 stroke engines;

6) a potential for use as a chemical reactor to produce power while simultaneously producing hydrogen-rich exhaust for use in solid oxide fuel cells [6].

\section{Prior WVU Research}

CIBAI combustion has been examined in detail at WVU as part of doctoral dissertation research by Fernando Echavarria in 2005-06 [1]. Of particular interest to the proposed new research is the system with which Echavarria simulated the patent's CCV concept. As shown in Figure 4, bottled high pressure air (1) was regulated (2) to a desired air-only TDC simulated pressure. A three way valve (3) was used to fix the injection air mass which was then preheated (4) in the exhaust of the CFR engine (7). An electronic control board (8) featuring adjustable event initiation and duration controlled the solenoid valve (5) injection of high pressure/high temperature air. Backflow into the injection unit was prevented using a specially designed check valve (6) which was fitted to the existing sparkplug hole through which the air injection entered the cylinder. 


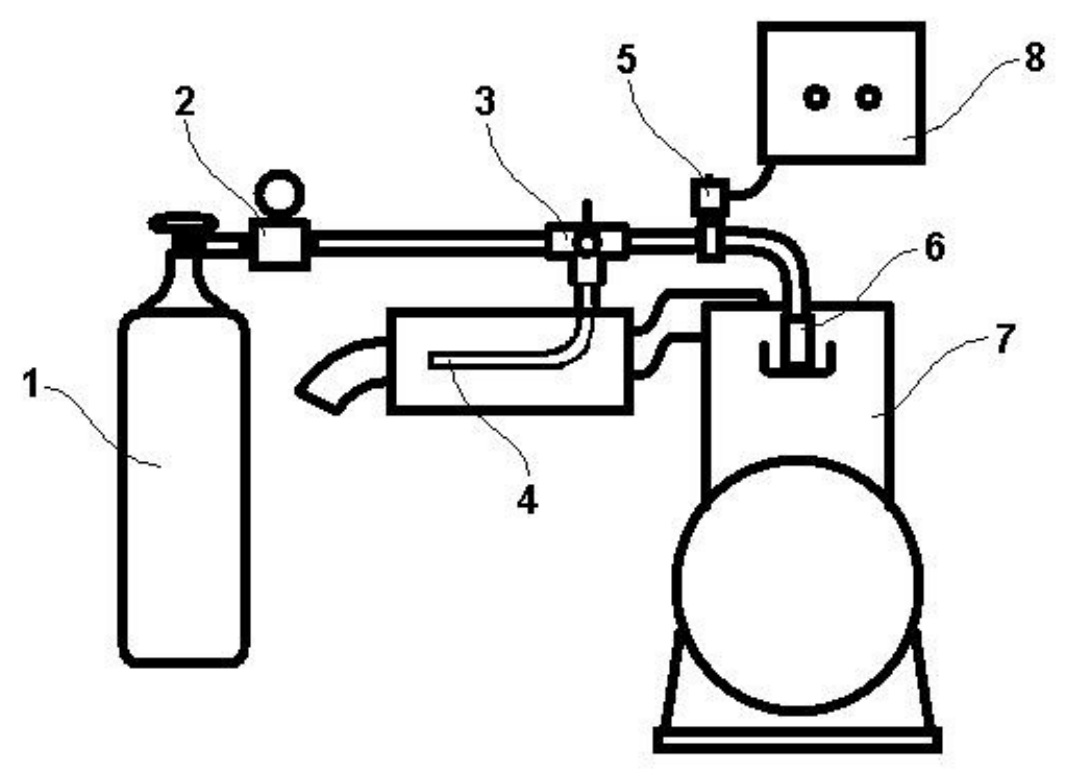

Figure 4: The air injection system used by Echavarria simulated actual CCV operation.

Echavarria noted in concluding remarks of his dissertation abstract (italics added), "Experimental and numerical model results indicated that ignition is readily achieved by air injection (CIBAI) in a CFR engine using the proper air injection system and proper air injection timing strategy."

The final phrase truly indicates the next challenge of the CIBAI cycle: design of the "proper air injection" apparatus and associated control system. 


\section{Relevant Valve Studies}

Much research has been done regarding the analysis of valve flow. Gault et al. [24] have demonstrated a simple technique of estimating mass flow rates through an orifice into a fixed volume tank by measuring instantaneous tank pressure, $\mathrm{p}_{\text {tank }}$, ambient air temperature, $\mathrm{T}_{\mathrm{amb}}$, instantaneous tank temperature $\mathrm{T}_{\text {tank, }}$, and tank wall temperature, $\mathrm{T}_{\text {wall }}$ [24]. By comparing the state variables of the tank before and after valve operation it is possible to determine the total mass that has entered the tank such that

$$
m_{i n}=\frac{V}{R_{\text {air }}}\left[\left(\frac{p}{T}\right)_{\operatorname{tank}, f}-\left(\frac{p}{T}\right)_{\operatorname{tank}, i}\right]
$$

The resultant value should be the same as the cumulative summation of mass flow rates including any heat loss effect such that

$$
m_{\text {cum }}=\left(\frac{\Delta t}{c_{p} T_{\text {amb }}}\right) \sum_{t=0}^{t=n}\left[\frac{\forall}{(\gamma-1)}\left(\frac{d p}{d t}\right)_{\text {tank }}+h A_{\text {wall }}\left(T_{\text {tank }}-T_{\text {wall }}\right)\right] .
$$

Using the transient tank pressure and temperature data, sampled at a constant frequency of $1 / \Delta \mathrm{t} \mathrm{Hz}$, it is possible to iteratively determine an appropriate value of heat transfer coefficient $\mathrm{h}$ (note that this method can yield either a time varying or a constant value of h). Once the heat transfer coefficient has been determined for a given system, the instantaneous mass flow rate at time $t_{i+1}$ through the orifice can be determined as

$$
\dot{m}(t)_{i+1}=\frac{\forall\left(p_{i+1}-p_{i}\right)_{\text {tank }}}{\Delta t(\gamma-1) c_{p} T_{\text {amb }}}+h A_{\text {wall }}\left(T_{\text {tank }}-T_{\text {wall }}\right)_{i+1}
$$

This expression can then be compared to the predicted theoretical mass flow rate based on isentropic compressible flow to produce an orifice discharge coefficient. This scheme worked best with an assumed constant $\mathrm{h}$ for the high pressure differential regime, but 
under low pressure differential the transient heat transfer coefficient was determined to work best [24].

Wedge flow meter correlations such as those presented by Yoon et al. may offer another way to determine the instantaneous flow rate through any outflow region of pipe [32]. For flows within the Reynolds number range of $1.2 \times 10^{4}$ to $3.8 \times 10^{5}$, the researchers were able to show a highly correlated nonlinear curve fit to several $90^{\circ}$ wedge restrictions. A schematic view of flow meter used in their research apparatus is presented in Figure 5.

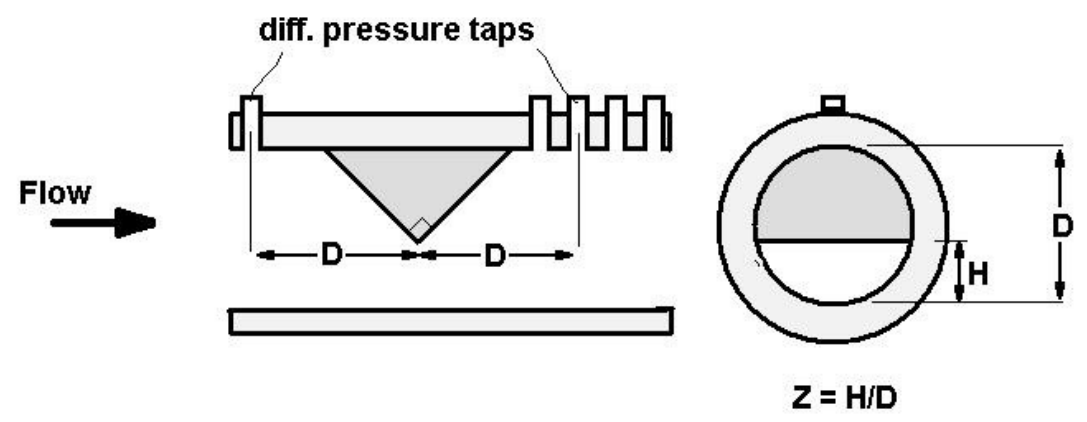

Figure 5: The wedge flow meter used in experimental correlations by Yoon et al.

According to the tests performed for values of $Z$ between 0.3 and 0.7 , the flow $C_{d}$ can be found according to the empirical formula

$$
C_{d}(Z)=0.9136 Z^{0.1303}-23.363 Z^{4}+50.378 Z^{3}-11.062 Z-1.1055 ; \quad Z \in[0.3,0.7]
$$

Given the empirical discharge coefficient, the mass flow rate can be found using the pressure differential and downstream temperature applied to the theoretical isentropic compressible flow-based mass flow rate. The established mass flow rate can then be compared to the theoretical mass flow rate for the $\mathrm{CCV}$ to identify the $\mathrm{CCV}$ discharge coefficient. It is worth noting that Yoon et al. [114] published the formula for the wedge shaped area of the flow meter as 


$$
A_{\text {wedge }}=\frac{\pi m D^{2}}{4}
$$

where the wedge-to-pipe area ratio, $\mathrm{m}$, is given as

$$
m=\frac{1}{\pi}\left[\cos ^{-1}(1-2 Z)-2(1-2 Z) \sqrt{Z(1-Z)}\right] .
$$

In the somewhat reversed situation of the research of Gault et al., Arkhipov et al. [31] have investigated the quasi-steady behavior of gas exhaust from a receiver, the likes of which might be found aboard space vehicles for the purpose of attitude microadjustments. Beginning with a hypothetical thermally insulated pressure tank, a valve is quickly (though not instantaneously) opened to allow gas exhaustion to occur. The researchers rather elegantly proceed by first estimating a nozzle starting time, defined as the longer of either time required for propagation of the first chamber rarefaction waves or the time for the system of shock waves in the nozzle to stabilize. They suggest that times as short as $\mathrm{t}=10^{-4}$ seconds are within the range of acceptable quasi-steady analysis of practical systems involving transitional behavior (e.g., rapid valve opening and closing). Next, the process considers a rapid asymptotic approach to a steady discharge coefficient starting from zero and the resulting exponentially dependent gas dynamic behavior during this transition time. Using a time increment just one order of magnitude lower than the predicted stabilization time, the researchers were able to accurately depict experimentally validated high pressure exhaust from a nozzle undergoing rapid opening. Highlights of the research, as it pertains to the proposed CCV study, are evident in the high speed transition of the nozzle opening, the short overall time scales ( $\tau \approx 1 \mathrm{msec}$ ), and the special attention to the asymptotic behavior of the nozzle discharge coefficient. 
Perhaps the most relevant of all recent studies are presented in two separate papers by Idris and Pullen [22] and Idris et al. [23], both of which address the behavior of real flow incidence angles in rotating valves. Their work centered on a systematic approach to correlating several sources of flow modification in a rotating valve such as effects based on:

- Reynolds number

- orifice inlet corner radiusing

- orifice length

- orifice inlet corner chamfering

- pressure differential

- pumping behavior

- incidence angle

- orifice inclination angle

- preswirl and inlet cross-flow

Valve discharge coefficient is offered by the researchers as the guiding flow modification factor. Ultimately, they provide a series of formulae by which future researchers and designers may estimate potential valve flow rates as a cumulative function of the effects of orifice and valve geometry, gas state variables, and orifice rotational speeds. Ranges of values for the experimental and numerical tests include rotational speeds from 0 (static) to $21,000 \mathrm{RPM}$, inclination angles from $0^{\circ}$ to $30^{\circ}$, Reynolds numbers from $4 \times 10^{4}$ to $1.4 \times 10^{5}$, incidence angles from $-30^{\circ}$ to $60^{\circ}$, radius r/d values from 0.1 to 0.36 , chamfer $w / d$ values from 0 to 0.25 , orifice length $L / d$ values 
from 0 to 10 , and pressure ratios from 1 to 2 (high enough to incorporate choked flow conditions). 


\section{Chapter 3: CIBAI Cold Air Standard Analysis}

Although detailed thermodynamic analysis of the CIBAI cycle has been performed by Echavarria in his earlier work, his findings must be corroborated by a second thorough analysis to identify theoretical benefits and limitations of the CIBAI cycle. Adopting the same cold air standard (CAS) assumptions proposed during the first analysis [1], the following assumptions have been made:

1) Air, fixed in mass and considered ideal in behavior, is the only working fluid within the closed system.

2) The complex combustion process is modeled as transfer of heat into the system.

3) Exhaust of the products of combustion is modeled as a transfer of heat out of the system, thus completing the cycle.

4) Every heat addition or rejection process occurring during the cycle is internally reversible.

5) The specific heats of the working fluid are considered constant throughout the cycle.

6) The air at the beginning of the cycle (state 1) has a temperature and pressure of $25^{\circ} \mathrm{C}(298 \mathrm{~K})$ and 1 atmosphere, respectively.

7) Opening of the CCV occurs instantaneously (from state 2 , initial to state 2, final).

8) Energy in all its forms is evenly distributed between the two cylinders instantaneously at state 2 , final.

These assumptions are readily seen in the thermodynamic diagrams of Figure 6. 

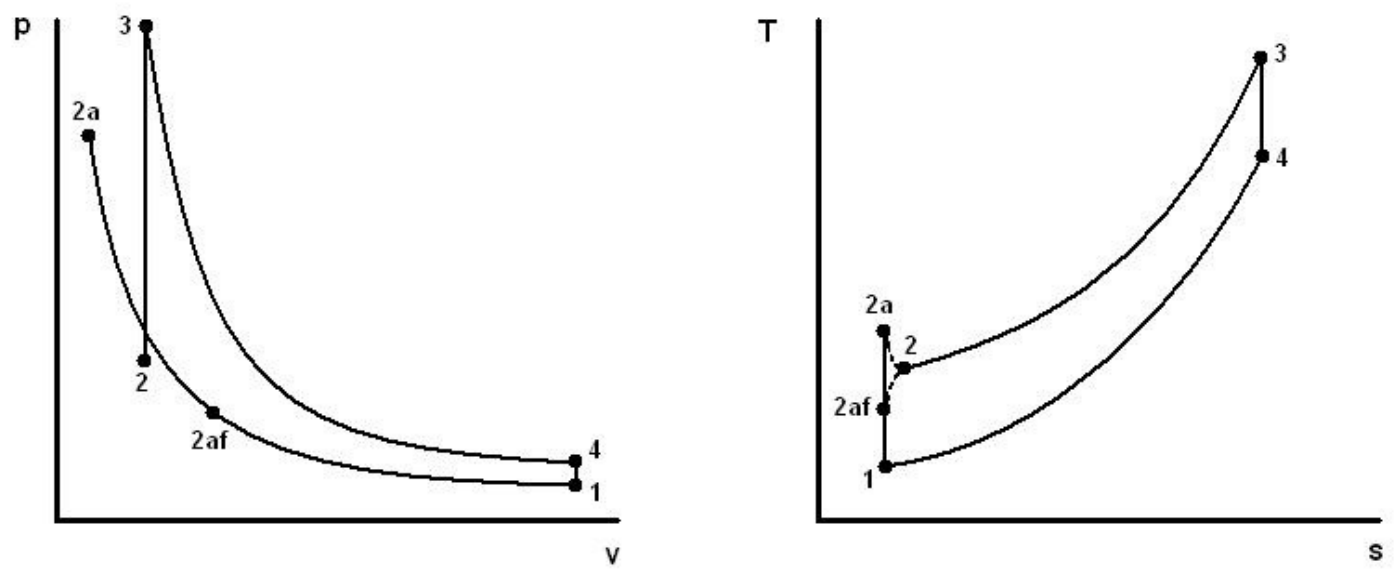

Figure 6: The ideal cold air standard CIBAI cycle, shown producing net work out in P-v and T-s diagrams.

The first part of analysis seeks to establish an expression of thermal efficiency of the CIBAI cycle. Beginning with the definition of thermal efficiency as the ratio of net work produced by a cycle to heat addition required, or

$$
\eta_{t h}=\frac{W_{n e t}}{Q_{i n}}
$$

it is possible to show that

$$
\eta_{t h}=\frac{Q_{\text {in }}-Q_{o u t}}{Q_{\text {in }}}=1-\frac{Q_{\text {out }}}{Q_{\text {in }}}
$$

The simplified combustion and exhaust processes of assumptions 2 and 3 lead to

$$
\begin{aligned}
& Q_{\text {in }}=U_{3}-U_{2}=m_{3} u_{3}-m_{2} u_{2}=m_{3} c_{v, 3} T_{3}-m_{2} c_{v, 2} T_{2} \\
& Q_{\text {out }}=U_{4}-U_{1}=m_{4} u_{4}-m_{1} u_{1}=m_{4} c_{v, 4} T_{4}-m_{1} c_{v, 1} T_{1}
\end{aligned}
$$

and from assumptions 1 and 5,

$$
\begin{aligned}
& m_{1}=m_{2}=m_{3}=m_{4}=m ; \\
& c_{v, 1}=c_{v, 2}=c_{v, 3}=c_{v, 4}=c_{v} ;
\end{aligned}
$$

so that 


$$
\begin{aligned}
& Q_{i n}=m c_{v}\left(T_{3}-T_{2}\right) ; \\
& Q_{\text {out }}=m c_{v}\left(T_{4}-T_{1}\right) ;
\end{aligned}
$$

and

$$
\eta_{t h}=1-\frac{Q_{o u t}}{Q_{\text {in }}}=1-\frac{m c_{v}\left(T_{4}-T_{1}\right)}{m c_{v}\left(T_{3}-T_{2}\right)}=1-\frac{\left(T_{4}-T_{1}\right)}{\left(T_{3}-T_{2}\right)}
$$

An improvement to the last equation can be made by forming dimensionless state temperatures as ratios to the known intake (state 1) temperature. This simplification leads to

$$
\eta_{t h}=1-\frac{\left[\left(\frac{T_{4}}{T_{1}}\right)-\left(\frac{T_{1}}{T_{1}}\right)\right]}{\left[\left(\frac{T_{3}}{T_{1}}\right)-\left(\frac{T_{2}}{T_{1}}\right)\right]}=1-\frac{\left(\frac{T_{4}}{T_{1}}\right)-1}{\left(\frac{T_{3}}{T_{1}}\right)-\left(\frac{T_{2}}{T_{1}}\right)} ;
$$

If a new variable, $\mathrm{X}$, is introduced which represents the peak dimensionless temperature of the cycle such that

$$
X=\frac{T_{3}}{T_{1}}
$$

it is now possible to express the thermal efficiency as

$$
\eta_{t h}=1-\frac{\left(\frac{T_{4}}{T_{1}}\right)-1}{X-\left(\frac{T_{2}}{T_{1}}\right)}
$$

Assumption 4 implies isentropic compression from state 1 to state 2 and similar expansion from state 3 to state 4 . These conditions coupled with the ideal gas behavior of assumption 1 allow the use of the generic isentropic relations

$$
\left(\frac{T_{A}}{T_{B}}\right)=\left(\frac{p_{A}}{p_{B}}\right)^{\frac{k-1}{k}}=\left(\frac{v_{B}}{v_{A}}\right)^{k-1}
$$


where $\mathrm{k}$ is the specific heat ratio, $\mathrm{k}=\mathrm{c}_{\mathrm{p}} / \mathrm{c}_{\mathrm{v}}$. Examination of the dimensionless state 4 temperature shows that

$$
\frac{T_{4}}{T_{1}}=\frac{T_{3}}{T_{1}}\left(\frac{T_{4}}{T_{3}}\right)=X\left(\frac{\forall_{3}}{V_{4}}\right)^{k-1}
$$

Additionally,

$$
\frac{V_{4}}{V_{3}}=\frac{V_{B D C, a}+V_{B D C, a f}}{V_{T D C, a}+V_{T D C, a f}}
$$

where the subscripts "a" and "af" denote the air-only and air-fuel cylinders, respectively. Let another new variable, VR, be introduced such that

$$
\forall_{B D C, a}=V R V_{B D C, a f}
$$

in which VR becomes a simultaneous expression of both initial volume and initial mass ratios of air-only to air-fuel cylinders. Employing the compression ratio (CR) expressions,

$$
\begin{aligned}
& V_{B D C, a}=C R_{a} V_{T D C, a} \\
& \forall_{B D C, a f}=C R_{a f} \forall_{T D C, a f}
\end{aligned}
$$

and manipulating the volume expressions at states 3 and 4 yields

$$
\begin{aligned}
& V_{3}=V_{T D C, a}+V_{T D C, a f}=\frac{V_{B D C, a}}{C R_{a}}+\frac{V_{B D C, a f}}{C R_{a f}}=\frac{V R V_{B D C, a f}}{C R_{a}}+\frac{V_{B D C, a f}}{C R_{a f}}=V_{B D C, a f}\left(\frac{V R}{C R_{a}}+\frac{1}{C R_{a f}}\right) ; \\
& V_{4}=V_{B D C, a}+\forall_{B D C, a f}=V R V_{B D C, a f}+V_{B D C, a f}=V_{B D C, a f}(1+V R) ; \\
& \frac{V_{3}}{V_{4}}=\frac{\forall_{B D C, a f}\left(\frac{V R}{C R_{a}}+\frac{1}{C R_{a f}}\right)}{V_{B D C, a f}(1+V R)}=\frac{\left(\frac{V R}{C R_{a}}+\frac{1}{C R_{a f}}\right)}{(1+V R)}=\frac{\left(\frac{V R C R_{a f}+C R_{a}}{C R_{a} C R_{a f}}\right)}{(1+V R)}=\frac{C R_{a}+V R C R_{a f}}{C R_{a} C R_{a f}(1+V R)}
\end{aligned}
$$

thus 


$$
\frac{T_{4}}{T_{1}}=X\left[\frac{C R_{a}+V R C R_{a f}}{C R_{a} C R_{a f}(1+V R)}\right]^{k-1}
$$

The dimensionless temperature at state 2 requires a bit more work. Solution of the value depends mainly upon assumptions 7 and 8 . If no work is done on or by the system during the adiabatic opening of the $\mathrm{CCV}$, and if the mass is assumed to flow instantaneously so that specific volume (thus density) remain constant during $\mathrm{CCV}$ operation, then conservation of energy within the fluid maintains

$$
\left.\frac{d E}{d t}\right|_{s y s}=\dot{W}-\dot{Q}+\sum_{\text {in }} m\left(u+\frac{V^{2}}{2}+z g\right)-\sum_{\text {out }} m\left(u+\frac{V^{2}}{2}+z g\right)=0
$$

Elimination of nearly every term except internal energies from state 2, initial to state 2, final yields the energy balance

$$
\begin{aligned}
& m_{2 a} u_{2 a}+m_{2 a f} u_{2 a f}-m_{2} u_{2}=m_{2 a} c_{v, 2 a} T_{2 a}+m_{2 a f} c_{v, 2 a f} T_{2 a f}-m_{2} c_{v, 2} T_{2}=0 ; \\
& T_{2}=\frac{\left(m_{2 a} c_{v, 2 a} T_{2 a}+m_{2 a f} c_{v, 2 a f} T_{2 a f}\right)}{m_{2} c_{v, 2}}=\frac{\left(m_{2 a} T_{2 a}+m_{2 a f} T_{2 a f}\right)}{m_{2}}
\end{aligned}
$$

Continuity and a closer inspection of VR help in making the next manipulations,

$$
\begin{gathered}
\frac{T_{2}}{T_{1}}=\frac{\left(m_{2 a} T_{2 a}+m_{2 a f} T_{2 a f}\right)}{m_{2} T_{1}}=\left(\frac{m_{2 a}}{m_{2}}\right)\left(\frac{T_{2 a}}{T_{1}}\right)+\left(\frac{m_{2 a f}}{m_{2}}\right)\left(\frac{T_{2 a f}}{T_{1}}\right) ; \\
m_{2}=m_{2 a}+m_{2 a f}=m_{1 a}+m_{1 a f}=\rho_{1}\left(V_{B D C, a}+V_{B D C, a f}\right) ; \\
\left(\frac{m_{2 a}}{m_{2}}\right)=\left(\frac{V_{B D C, a}}{V_{B D C, a}+V_{B D C, a f}}\right)=\left(\frac{V R V_{B D C, a f}}{V R V_{B D C, a f}+V_{B D C, a f}}\right)=\left(\frac{V R}{1+V R}\right) \\
\left(\frac{m_{2 a f}}{m_{2}}\right)=\left(\frac{V_{B D C, a f}}{V_{B D C, a}+V_{B D C, a f}}\right)=\left(\frac{V_{B D C, a} / V R}{V_{B D C, a}+V_{B D C, a} / V R}\right)=\left(\frac{1 / V R}{1+1 / V R}\right)=\left(\frac{1}{1+V R}\right) ;
\end{gathered}
$$




$$
\begin{aligned}
& \left(\frac{T_{2 a}}{T_{1}}\right)=\left(\frac{V_{B D C, a}}{V_{T D C, a}}\right)^{k-1}=C R_{a}^{k-1} ; \\
& \left(\frac{T_{2 a f}}{T_{1}}\right)=\left(\frac{V_{B D C, a f}}{V_{T D C, a f}}\right)^{k-1}=C R_{a f}^{k-1} ;
\end{aligned}
$$

finally providing the ratio

$$
\frac{T_{2}}{T_{1}}=\left(\frac{V R}{1+V R}\right) C R_{a}^{k-1}+\left(\frac{1}{1+V R}\right) C R_{a f}^{k-1}=\frac{\left(V R C R_{a}^{k-1}+C R_{a f}^{k-1}\right)}{(1+V R)}
$$

The result of these manipulations is ultimately an expression of thermal efficiency for the CIBAI cycle operating under the assumption previously listed as a function of peak dimensionless temperature $\mathrm{X}$, initial cylinder volume ratio VR, cylinder compression ratios $\mathrm{CR}_{\mathrm{a}}$ and $\mathrm{CR}_{\mathrm{af}}$, and the specific heat ratio $\mathrm{k}$. The final equation is

$$
\eta_{t h, C I B A I}=1-\frac{X\left[\frac{C R_{a}+V R C R_{a f}}{C R_{a} C R_{a f}(1+V R)}\right]^{(k-1)}-1}{\left[X-\frac{\left(V R C R_{a}^{k-1}+C R_{a f}^{k-1}\right)}{(1+V R)}\right]}
$$

Comparison of the thermal efficiency of the CIBAI cycle to a similar Otto cycle is done easily by setting the Otto cycle maximum compression ratio equal to that of the maximum air-fuel cylinder compression ratio of the CIBAI engine. This is a fair comparison in the sense that both cycles are limited by the maximum amount of compression that can take place before the associated heating incites engine knock. Applying the air-fuel compression ratio to the familiar expression for Otto thermal efficiency yields

$$
\eta_{t h, O t t o}=1-C R_{a f}^{1-k}
$$


The method for fair comparison between comparable CIBAI and Diesel cycles is not as easily reached, but is still possible. Focus must remain on the two major limiting factors in both cycles, namely the dimensionless peak temperature, $\mathrm{X}$, and the maximum cylinder compression ratio, $\mathrm{CR}_{\mathrm{a}}$. Ascertaining Diesel thermal efficiency based on these values is first approached by recognizing the constant pressure heat addition process unique to the Diesel cycle. Specifically,

$$
p_{3}=p_{2}
$$

combined with the ideal gas behavior and fixed mass of the air as stated in assumption 1 lead to the relations

$$
\begin{aligned}
& p_{2} v_{2}=n R T_{2} ; \quad p_{3} v_{3}=n R T_{3} ; \\
& \frac{n R T_{2}}{v_{2}}=\frac{n R T_{3}}{v_{3}} ; \quad \frac{T_{2}}{T_{3}}=\frac{v_{2}}{v_{3}}=\frac{V_{2}}{V_{3}}
\end{aligned}
$$

which can be used in conjunction with the definition of the cut off ratio, $\mathrm{CO}$, to show

$$
C O=\frac{V_{3}}{V_{2}}=\frac{T_{3}}{T_{2}}=\frac{T_{3}}{T_{1}}\left(\frac{T_{1}}{T_{2}}\right)
$$

The isentropic compression process from states 1 to 2 allow $\mathrm{CO}$ to be expressed as

$$
C O=X\left(\frac{v_{2}}{v_{1}}\right)^{k-1}=X C R_{a}^{1-k}
$$

The familiar expression for thermal efficiency of the Diesel cycle in terms of the compression ratio and cut off ratio, can now be expressed using comparable variables of the CIBAI cycle, such that

$$
\eta_{t h, \text { Diesel }}=1-C R_{a}^{1-k}\left[\frac{C O^{k}-1}{k(C O-1)}\right]
$$

or more specifically, 


$$
\eta_{t h, \text { Diesel }}=1-C R_{a}^{1-k}\left[\frac{\left(X C R_{a}^{1-k}\right)^{k}-1}{k\left(X C R_{a}^{1-k}-1\right)}\right]
$$

Comparison of thermal efficiency over a range of $\mathrm{CR}_{\mathrm{a}}$ values is presented in Figure 7.

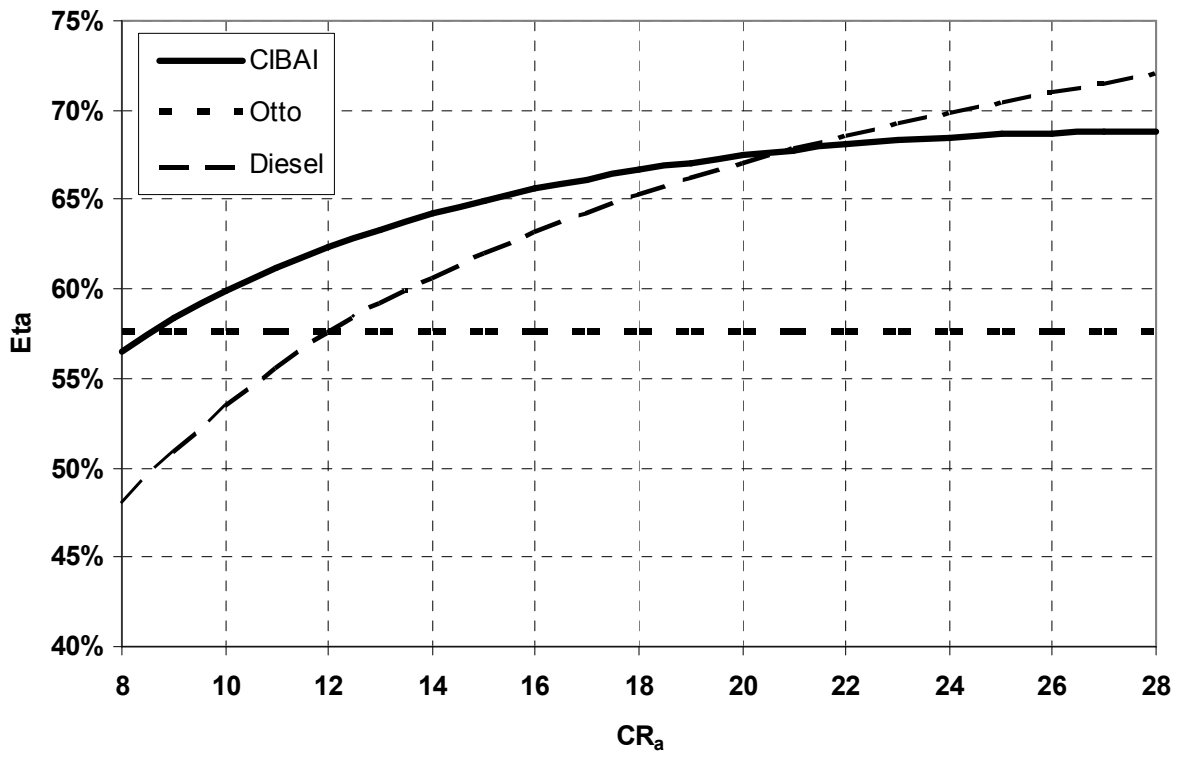

Figure 7: Thermal efficiency comparison of the CIBAI, Otto, and Diesel cycles operating under the following conditions: $\mathrm{CR}_{\mathrm{af}}=8.5, \mathrm{X}=5, \mathrm{VR}=11, \mathrm{k}=1.4,8<\mathrm{CR}_{\mathrm{a}}<28$.

Another key indicator of overall cycle performance in reciprocating piston engines is the mean effective pressure (MEP). Defined as the amount of work produced during a single complete cycle per unit volume displacement, MEP can be thought of as engine work density. In general, MEP can be written as

$$
M E P=\frac{W_{\text {net }}}{V_{\text {disp }}}
$$

A dimensionless comparison between the MEP's of each cycle seems most prudent and since the units are (as the name implies) in terms of force per unit area, the MEP of each cycle may be rendered dimensionless using the CAS pressure of 1 atmosphere $(101,325$ Pa). Beginning with the dimensionless CIBAI MEP, we may write 


$$
\frac{M E P_{C I B A I}}{p_{C A S}}=\frac{W_{\text {net }}}{p_{1} V_{\text {disp }}}=\frac{Q_{\text {in }}-Q_{\text {out }}}{p_{1} V_{\text {disp }}} ;
$$

Noting the ideal gas behavior and constant volume heat addition,

$$
\begin{aligned}
& Q_{\text {in }}=m c_{v}\left(T_{3}-T_{2}\right) ; \\
& Q_{\text {out }}=m c_{v}\left(T_{4}-T_{1}\right) ; \\
& p_{1}=\frac{m R T_{1}}{V_{1}} ; \\
& \frac{M E P_{C I B A I}}{p_{1}}=\frac{m c_{v}\left(T_{3}-T_{2}\right)-m c_{v}\left(T_{4}-T_{1}\right)}{\left(\frac{m R T_{1}}{V_{1}}\right) V_{\text {disp }}}=\frac{m c_{v}\left(T_{3}-T_{2}-T_{4}+T_{1}\right)}{m R T_{1}\left(\frac{V_{\text {disp }}}{V_{1}}\right)} ; \\
& \frac{M E P_{C I B A I}}{p_{1}}=\left(\frac{c_{v}}{R}\right) \frac{\left[\left(\frac{T_{3}}{T_{1}}\right)+1-\left(\frac{T_{2}}{T_{1}}\right)-\left(\frac{T_{4}}{T_{1}}\right)\right]}{\left(\frac{V_{\text {disp }}}{V_{1}}\right)}
\end{aligned}
$$

The dimensionless MEP at this stage has many recognizable ratios, but the volume ratio in the denominator of the expression needs a little more manipulation. Employing the various definitions already given for the compression ratios particular to the CIBAI cycle,

$$
\begin{aligned}
& \left(\frac{V_{d i s p}}{V_{1}}\right)=\frac{\left[\left(V_{B D C, a}+V_{B D C, a f}\right)-\left(V_{T D C, a}+V_{T D C, a f}\right)\right]}{\left(V_{B D C, a}+V_{B D C, a f}\right)}=1-\frac{\left(V_{T D C, a}+V_{T D C, a f}\right)}{\left(V_{B D C, a}+V_{B D C, a f}\right)} \\
& \left(\frac{V_{\text {disp }}}{V_{1}}\right)=1-\frac{\left(\frac{V_{B D C, a}}{C R_{a}}+\frac{V_{B D C, a f}}{C R_{a f}}\right)}{\left(V_{B D C, a}+V_{B D C, a f}\right)}=1-\frac{\left(\frac{V R V_{B D C, a f}}{C R_{a}}+\frac{V_{B D C, a f}}{C R_{a f}}\right)}{\left(V R V_{B D C, a f}+V_{B D C, a f}\right)}=1-\frac{V_{B D C, a f}\left(\frac{V R}{C R_{a}}+\frac{1}{C R_{a f}}\right)}{V_{B D C, a f}(V R+1)} ; \\
& \left(\frac{V_{\text {disp }}}{V_{1}}\right)=1-\frac{\left(\frac{V R C R_{a f}+C R_{a}}{C R_{a} C R_{a f}}\right)}{(V R+1)}=1-\frac{C R_{a}+V R C R_{a f}}{C R_{a} C R_{a f}(1+V R)}
\end{aligned}
$$

Also noting that

$$
\frac{c_{v}}{R}=\frac{c_{v}}{c_{p}-c_{v}}=\frac{1}{k-1}
$$


yields a final dimensionless CIBAI MEP expression as

$$
\frac{M E P_{C I B A I}}{p_{C A S}}=\left(\frac{1}{k-1}\right) \frac{\left[X+1-\left(\frac{T_{2}}{T_{1}}\right)-\left(\frac{T_{4}}{T_{1}}\right)\right]}{\left[1-\frac{C R_{a}+V R C R_{a f}}{C R_{a} C R_{a f}(1+V R)}\right]}
$$

in which all variables are known for a given set of cycle parameters.

As before, a fair comparison to the dimensionless MEP of the Otto cycle proceeds as

$$
\frac{M E P_{\text {Oto }}}{p_{C A S}}=\frac{m c_{v}\left(T_{3}-T_{2}\right)-m c_{v}\left(T_{4}-T_{1}\right)}{p_{1} \forall_{\text {disp }}} ;
$$

Using the same substitution methods demonstrated in the case of the CIBAI cycle, Otto MEP can now be expressed as

$$
\frac{M E P_{\text {Oto }}}{p_{\text {CAS }}}=\left(\frac{1}{k-1}\right) \frac{\left[X+1-\left(\frac{T_{2}}{T_{1}}\right)-\left(\frac{T_{4}}{T_{1}}\right)\right]}{\left(\frac{W_{\text {disp }}}{F_{1}}\right)}
$$

Further reduction of the remaining ratios,

$$
\begin{aligned}
& \left(\frac{T_{2}}{T_{1}}\right)=\left(\frac{v_{1}}{V_{2}}\right)^{k-1}=\left(\frac{V_{1}}{V_{2}}\right)^{k-1}=C R_{a f}^{k-1} ; \\
& \left(\frac{T_{4}}{T_{1}}\right)=\frac{T_{3}}{T_{1}}\left(\frac{T_{4}}{T_{3}}\right)=X\left(\frac{v_{3}}{v_{4}}\right)^{k-1}=X\left(\frac{V_{3}}{V_{4}}\right)^{k-1}=X C R_{a f}^{1-k} ; \\
& \left(\frac{V_{d i s p}}{V_{1}}\right)=\left(\frac{V_{B D C, a f}-V_{T D C, a f}}{V_{B D C, a f}}\right)=1-\frac{V_{T D C, a f}}{V_{B D C, a f}}=1-C R_{a f}^{-1}=\frac{C R_{a f}-1}{C R_{a f}}
\end{aligned}
$$

yields

$$
\frac{M E P_{\text {Otoo }}}{p_{C A S}}=\left(\frac{1}{k-1}\right) \frac{\left[(X+1)-C R_{a f}^{k-1}-X C R_{a f}^{1-k}\right]}{\left(\frac{C R_{a f}-1}{C R_{a f}}\right)}=\left(\frac{C R_{a f}}{k-1}\right) \frac{\left[X+1-C R_{a f}^{k-1}-X C R_{a f}^{1-k}\right]}{\left(C R_{a f}-1\right)}
$$

and finally gives an expression of the dimensionless Otto cycle MEP as 


$$
\frac{M E P_{\text {Otoo }}}{p_{C A S}}=\frac{C R_{a f}\left(1+X-C R_{a f}^{k-1}-X C R_{a f}^{1-k}\right)}{(k-1)\left(C R_{a f}-1\right)}
$$

A similar investigation of dimensionless Diesel MEP begins with the expression

$$
\frac{M E P_{\text {Diesel }}}{p_{C A S}}=\frac{m c_{p}\left(T_{3}-T_{2}\right)-m c_{v}\left(T_{4}-T_{1}\right)}{p_{1} Y_{\text {disp }}} ;
$$

Again, using techniques already demonstrated, the expression can be written

$$
\frac{M E P_{\text {Diesel }}}{p_{\text {CAS }}}=\left(\frac{1}{k-1}\right) \frac{\left[k X-k C R_{a}^{k-1}-\left(\frac{T_{4}}{T_{1}}\right)+1\right]}{\left(1-C R_{a}^{-1}\right)}
$$

in which the dimensionless state 4 temperature is still unknown. This is readily solved as

$$
\begin{gathered}
C O=\frac{V_{3}}{V_{2}} ; C R_{a}=\frac{V_{1}}{V_{2}} ; \quad \forall_{1}=\forall_{4} ; \\
C O=C R_{a}\left(\frac{\forall_{3}}{V_{1}}\right)=C R_{a}\left(\frac{V_{3}}{V_{4}}\right)=C R_{a}\left(\frac{T_{4}}{T_{3}}\right)^{\frac{1}{k-1}}=C R_{a}\left[\left(\frac{T_{4}}{T_{1}}\right)\left(\frac{T_{1}}{T_{3}}\right)\right]^{\frac{1}{k-1}} ; \\
\left(\frac{C O}{C R_{a}}\right)=\left[\left(\frac{T_{4}}{T_{1}}\right)\left(\frac{T_{1}}{T_{3}}\right)\right]^{\frac{1}{k-1}} ;\left(\frac{C O}{C R_{a}}\right)^{k-1}=\left(\frac{T_{4}}{T_{1}}\right)\left(\frac{T_{1}}{T_{3}}\right) ; \\
\left(\frac{T_{4}}{T_{1}}\right)=X\left(\frac{C O}{C R_{a}}\right)^{k-1}
\end{gathered}
$$

In this last expression of the dimensionless state 4 temperature, all variables are known. At last, by substitution,

$$
\frac{M E P_{\text {Diesel }}}{p_{C A S}}=\left(\frac{1}{k-1}\right) \frac{\left[k X-k C R_{a}^{k-1}-X\left(\frac{C O}{C R_{a}}\right)^{k-1}+1\right]}{\left(1-C R_{a}^{-1}\right)}
$$


Figure 8 shows the comparison of ideal MEP values, again presented over a range or $\mathrm{CR}_{\mathrm{a}}$.

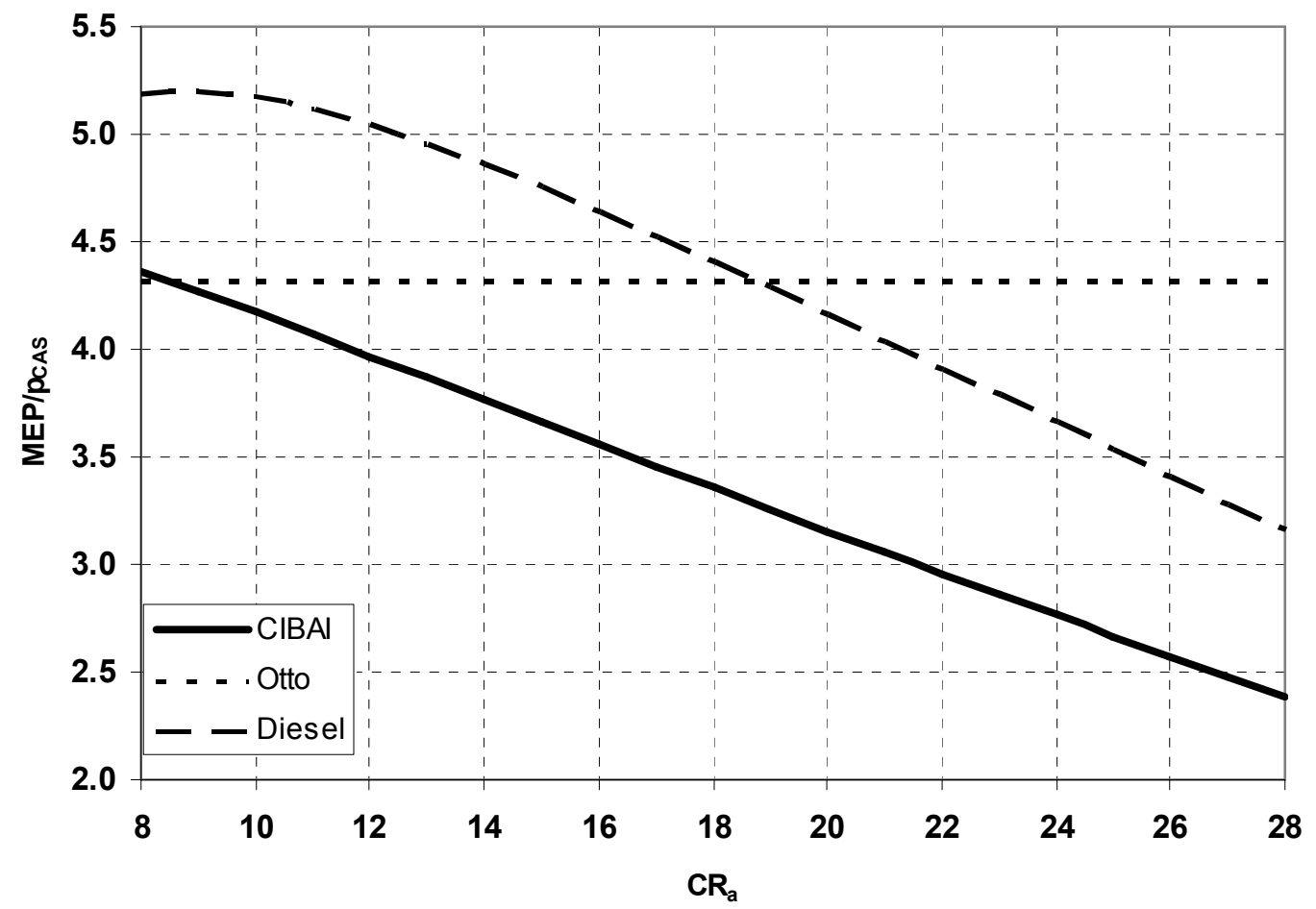

Figure 8: Comparison of dimensionless MEP for CIBAI, Otto, and Diesel cycles for conditions: CR $_{\text {af }}$ $=8.5, \mathrm{X}=5, \mathrm{VR}=11, \mathrm{k}=1.4,8<\mathrm{CR}_{\mathrm{a}}<28$.

A full evaluation of the benefits of the cycles in comparison can not be performed without the integration of maximum combustion speeds. 


\section{Chapter 4: Non-Steady Valve Flow Modeling}

\section{Minimum Duct Losses}

Because the goal of the research seeks to quantify CCV duct losses, it seems appropriate to investigate the theoretical "best case" scenario for purposes of comparison. In an ideal valve, all fluid movement occurs unhindered such that the fluid maintains all its initial energy without loss as mass is transported. This ideal case is of course never possible since it requires "slip" at the duct walls. Steady state kinetic energy dissipation through viscous shear wall heating has been exhaustively studied, resulting in the wellknown Moody chart for fully developed flow which describes the duct friction factor, $\mathrm{f}$, as a function of flow Reynolds number and wall relative roughness [4]. Friction factor is critical in evaluating flow inefficiency which can be represented as a static pressure drop,

$$
\Delta p=f\left(\frac{1}{2} \rho V^{2}\right) \frac{l}{D_{h}}
$$

where 1 is the length of the duct. In general for laminar flow, the friction factor only depends on flow Reynolds number such that

$$
f_{\text {lam }}=\frac{64}{\mathrm{Re}_{D}}, \quad \operatorname{Re}_{D}<2300
$$

Flow regimes where the Reynolds number is greater than $\sim 2300$ results in a new expression for friction factor which incorporates the strong dependence on wall surface roughness. The Colebrook formula for turbulent pipe flow is

$$
\frac{1}{\sqrt{f}}=-2 \log \left(\frac{\varepsilon / D_{h}}{3.7}+\frac{2.51}{\operatorname{Re}_{D} \sqrt{f}}\right), \quad \operatorname{Re}_{D}>2300 .
$$


The turbulent case requires some type of iterative scheme to solve for $f$, but the Colebrook formula is easily modified to a form befitting of standard Jacobian iteration such that

$$
f_{i+1}=\left[-2 \log \left(\frac{\varepsilon / D_{h}}{3.7}+\frac{2.51}{\operatorname{Re}_{D} \sqrt{f_{i}}}\right)\right]^{-2}
$$

This particular form rapidly converges, typically reaching relative error values of less that $0.01 \%$ within 5 iterations.

As previously stated, these methods establish a best case loss scenario, where the brunt of entrance effects is already dissipated to zero and no transient inertial effects are present. Thus, the value of pressure loss granted through this process serves as a type of Carnot efficiency as applied to orifice flow. 


\section{Compressible Flow Theory}

The general fluid flow within an engine is nearly always highly compressible in nature, thus accurate numerical modeling must incorporate this behavior. The boundary conditions used to depict compressible gas flow through the $\mathrm{CCV}$ and intake and exhaust ports are shown in Figure 9.

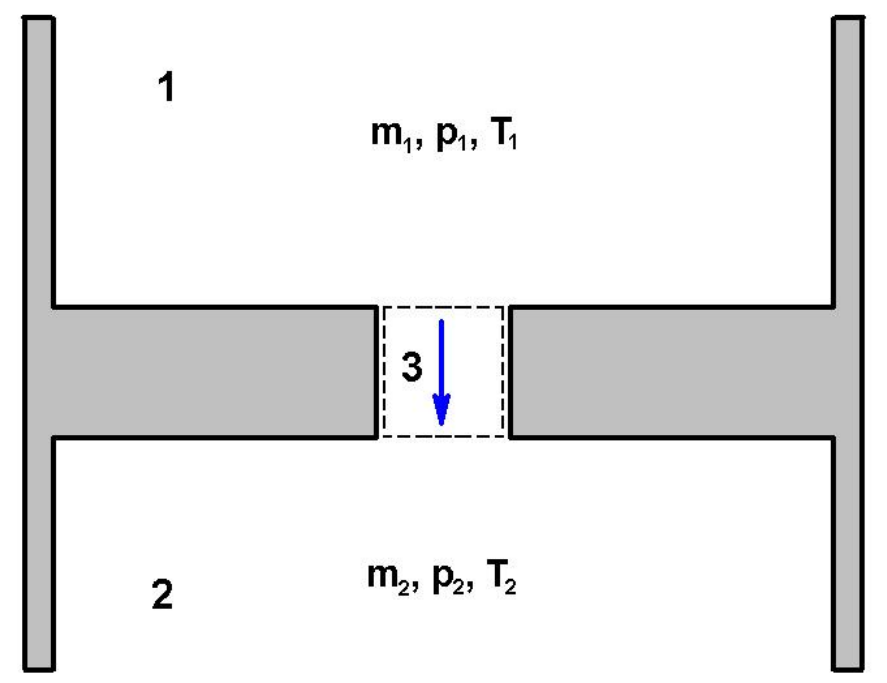

Figure 9: Compressible flow equations can be applied to the simplified conditions above in which region 3 represents the fully open $\mathrm{CCV}$.

Initially, regions 1,2 , and 3 are all at rest and the static pressures and temperatures within each region are by definition stagnation or total temperatures and pressures. Assuming that region 1 exhibits some increase in total pressure and/or temperature, equilibrium in the system is disturbed and fluid mass tends to move through the connecting region 3 into region 2 in a natural response to restore equilibrium. According to the quasi-steady model,

$$
\left.\frac{d m}{d t}\right|_{3}=\left.\frac{d E}{d t}\right|_{3} \equiv 0
$$


and by continuity and conservation of energy in a perfectly insulated system,

$$
\left.\frac{d m}{d t}\right|_{1}=-\left.\frac{d m}{d t}\right|_{2},\left.\quad \frac{d E}{d t}\right|_{1}=-\left.\frac{d E}{d t}\right|_{2}
$$

respectively. Compressible flow equations can be applied to determine the instantaneous time rate of change of mass that leaves region 1 and enters region 2. The energy equation is applied to determine the corresponding time rate of change of energy in each region. Based on the above description of the quasi-steady scenario, it is possible to write

$$
\left.\frac{d m}{d t}\right|_{1}=\dot{m}_{1}=\left.\rho V\right|_{1} A_{3}=\left[\frac{p}{R T} V\right]_{1} A_{3}
$$

where $A_{3}$ represents the cross sectional area of region 3 normal to the direction of flow. Rewriting the velocity at 1 as

$$
V_{1}=M_{1} a_{1}=M_{1} \sqrt{\gamma R T_{1}}
$$

and noting that the local Mach number, M, of any flow may be expressed as

$$
M=\sqrt{\left(\frac{2}{\gamma-1}\right)\left[\left(\frac{p_{0}}{p}\right)^{\frac{(\gamma-1)}{\gamma}}-1\right]}, \quad p_{0} \geq p[5]
$$

it is possible to write the instantaneous mass flow rate leaving region 1 as

$$
\dot{m}_{1}=A_{3} \sqrt{\left(\frac{p_{1}^{2}}{R T_{1}}\right)\left(\frac{2 \gamma}{\gamma-1}\right)\left[\left(\frac{p_{01}}{p_{1}}\right)^{\frac{(\gamma-1)}{\gamma}}-1\right]} .
$$

The addition of an expression for static temperature at location 1 ,

$$
T_{1}=T_{01}\left(\frac{p_{1}}{p_{01}}\right)^{\frac{(\gamma-1)}{\gamma}},
$$

yields 


$$
\dot{m}_{1}=A_{3} \sqrt{\left(\frac{p_{1}^{2}}{R T_{01}}\right)\left(\frac{p_{01}}{p_{1}}\right)^{\frac{(\gamma-1)}{\gamma}}\left(\frac{2 \gamma}{\gamma-1}\right)\left[\left(\frac{p_{01}}{p_{1}}\right)^{\frac{(\gamma-1)}{\gamma}}-1\right]} .
$$

The value of the expression above is perhaps not immediately obvious. However, with reference to Figure 9, if the flow is allowed to accelerate such that the boundary condition at the interface between regions $2 \& 3$ is static pressure equilibrium $\left(\mathrm{p}_{\text {interface }}=\right.$ $\mathrm{p}_{2}$ ), and the stagnation pressure and temperature of region 1 remain instantaneously unchanged $\left(\mathrm{p}_{1}=\mathrm{p}_{01}, \mathrm{~T}_{1}=\mathrm{T}_{01}\right)$, then the instantaneous mass flow rate through region 3 can be calculated explicitly as

$$
\dot{m}=A_{3} \sqrt{\left(\frac{p_{2}^{2}}{R T_{1}}\right)\left(\frac{p_{1}}{p_{2}}\right)^{\frac{(\gamma-1)}{\gamma}}\left(\frac{2 \gamma}{\gamma-1}\right)\left[\left(\frac{p_{1}}{p_{2}}\right)^{\frac{(\gamma-1)}{\gamma}}-1\right]}
$$

Unless the initial energy and mass in each region is forced to remain constant, this expression is only good for an infinitesimally short duration, $\Delta \mathrm{t}$, after which time the transported mass and energy changes the operating pressures and temperatures. It is also important to recognize that the maximum flow Mach number of the restriction of region 3 is limited to sonic Mach 1 (choked flow), where the mass flow rate becomes independent of downstream conditions and can be written

$$
\dot{m}_{\text {choked }}=\frac{p_{1} A_{3}}{\sqrt{T_{1}}} \sqrt{\frac{\gamma}{R}\left(\frac{2}{\gamma+1}\right)^{(\gamma+1) /(\gamma-1)}}
$$




\section{Quasi-Steady Flow Modeling}

A popular form of numerical modeling in which fluid mass accumulation between major engine components is neglected is referred to as quasi-steady modeling [2,3]. For the case of the CIBAI engine, the air-only and air-fuel cylinder volumes contain all the fluid mass within the engine. Air is allowed to enter at the intake port, and the incremental change in mass inflow is determined by the compressible flow equations $[2,5]$ and the selected time increment. The limiting factor of this method of modeling is its inability to predict variations in intake and exhaust (as well as CCV) flow phenomena based on full 3-D computational Fluid Dynamics (CFD) approaches [5]. Nonetheless, it is a widely accepted method of thermodynamics-based modeling. Quasi-steady codes used to indicate CIBAI engine behavior to date are presented in the appendix.

The basic approach of the method as applied to the CIBAI engine arrangement is outlined briefly as follows. Two "volumes" represented as mathematical points are associated with an array of time-dependent geometric configurations based on possible piston-cylinder arrangements, valve sizes and valve operating times. Initial conditions are assigned for each point, which in general start with air at ambient conditions. A constant crank speed is then assigned to the system, and the code begins to march in small time and angular increments. Monitoring the conditions within each "volume" requires a minimum of equations including conservation of energy, continuity, and for "cold" engines the ideal gas relations. Larger valves such as intake and exhaust ports are assumed to be capable of extremely rapid opening and closing times, but the specialized $\mathrm{CCV}$ is modeled according to the tested behavioral capabilities. Given the initial gas conditions the geometric evolution of the engine components, the thermodynamic 
properties of the quasi-steady gas, and the theoretical limits of the valve dynamics it is possible to model the engine at least to the point of determining some general qualitative aspects of the cycle. 


\section{Shock Tube Theory}

A relevant way to describe the valve scheme is by comparison to the wave system of a shock tube. The basic design of a simple shock tube is such that in an initial setup, an inviscid, perfect gas at high pressure is separated from an inviscid, perfect gas at low pressure by a thin diaphragm within a tube of infinite length and constant cross sectional area [35]. At a prescribed moment, the diaphragm is rapidly burst and the ensuing shock, compression, contact, and rarefaction waves disperse into the fluid in both directions. The resulting fluid properties can be predicted as functions of time and distance from the initial position of the diaphragm. Adding to the complexity of the transient behavior of such a system, a tube of finite length can be considered in which waves are reflected off end walls and subsequently reflected or refracted off one another (Figure 10). 


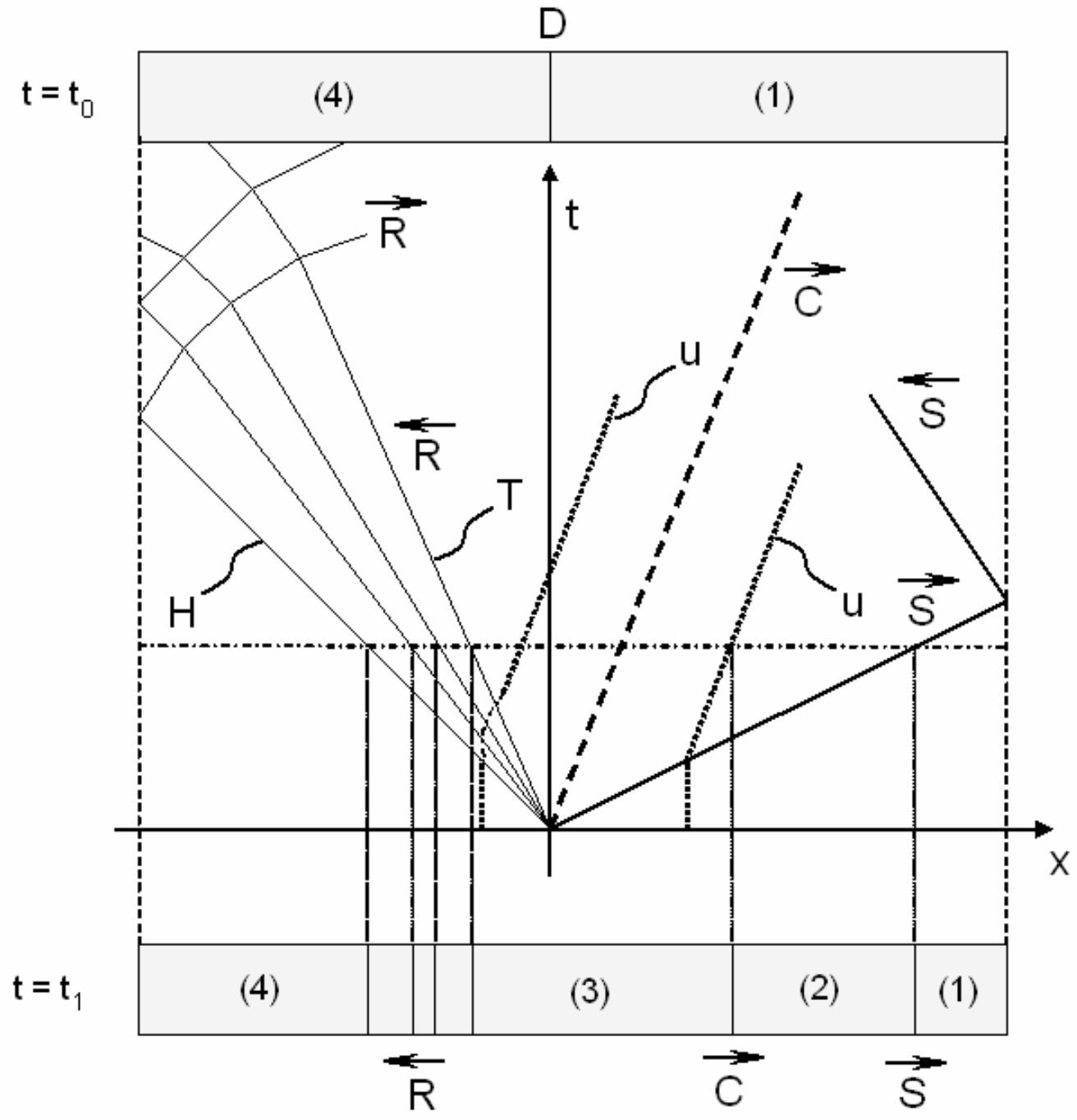

Figure 10: A shock tube wave system on the (x,t) plane (figure reproduced from [35]).

Following many of the suggested steps by Glass and Hall [35], a temporal development of the flow within the shock tube can be produced. Initially (at time, $\mathrm{t}<=$ $\mathrm{t}_{0}$ ), regions 1 and 4 are separated by the diaphragm (D) (Figure 11). Region 4 contains the high pressure gas and region 1 contains the relatively low pressure gas. At the instant of diaphragm rupture, the high pressure gradient apparent to the fluid at the new interface induces a shock wave system. This system starts with three simultaneous reactions: a 
shock wave, a contact front, and a rarefaction wave. Figure 12 shows the shock wave $\left(\mathrm{S}_{1}\right)$ moving rapidly into the gas at rest in region 1 . Just behind the shock, in region 2 , particles are accelerated to a fixed velocity, $\mathrm{u}_{2}$. In a manner analogous to falling sand that sheers from the face of a tall sand pile in sheets, particles are "sheeted" away from the high pressure gas in region 4 inside a rarefaction zone $\left(R_{1}\right)$. The head rarefaction wave (H, Figure 10), which always travels at the local speed of sound, enters region 4 where the particles at rest begin accelerating in the direction of region 1. The tail rarefaction wave (T, Figure 10) is defined by the location at which the particles have stopped accelerating and are moving at a constant velocity, $\mathrm{u}_{3}$. A critical boundary condition needed to establish equilibrium between the shocked region 2 and the sheered region 3 is that the velocities and pressures must be identical. This boundary condition indicates a contact front $\left(\mathrm{C}_{1}\right)$, moving at the same velocity as the particles in regions 2 and 3 that separates the two regions according to the difference in their remaining thermodynamic properties such that $\mathrm{T}_{2} \neq \mathrm{T}_{3}$ and $\rho_{2} \neq \rho_{3}$.

$$
\left(t=t_{0}\right)
$$

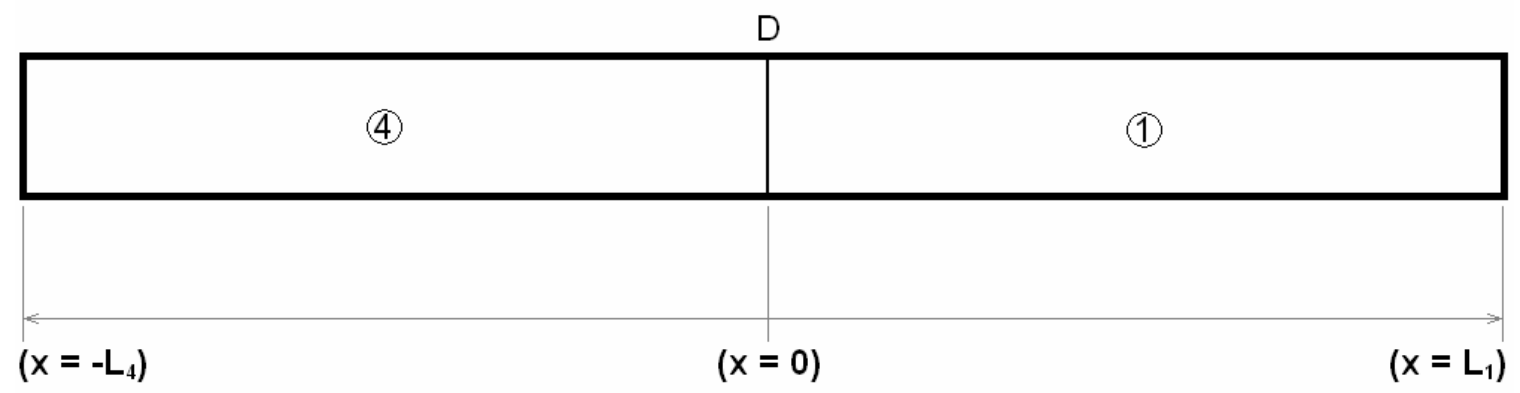

Figure 11: The shock tube at initial time, $t_{0}$ shows the high pressure region 4 separated from the low pressure region 1 by a thin diaphragm (D). 
$\left(t>t_{0}\right)$

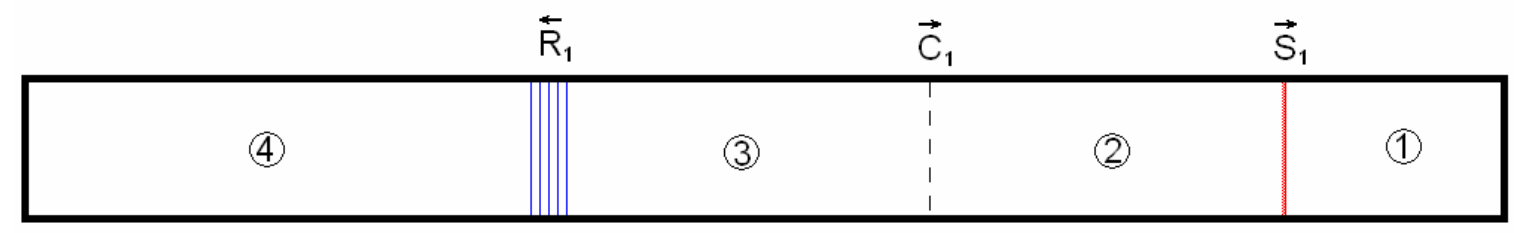

Figure 12: A starting shock wave $\left(S_{1}\right)$, contact front $\left(C_{1}\right)$, and rarefaction wave $\left(R_{1}\right)$ immediately develop upon rupture of the diaphragm.

Proceeding with calculations requires establishing a few defining parameters of the fluid based upon the known initial conditions of regions 1 and 4 . Glass suggests the following definitions:

$$
\begin{aligned}
& \alpha_{n} \stackrel{d}{=} \frac{\gamma_{n}+1}{\gamma_{n}-1}, \\
& \beta_{n} \stackrel{d}{=} \frac{\gamma_{n}-1}{2 \gamma_{n}} \\
& C_{v, n} \stackrel{d}{=} \frac{R_{n}}{\gamma_{n}-1}, \quad C_{p, n} \stackrel{d}{=} \frac{\gamma_{n} R_{n}}{\gamma_{n}-1}, \\
& E_{m n}=\frac{d\left(C_{v} T\right)_{m}}{\left(C_{v} T\right)_{n}} \\
& P_{m n} \stackrel{d}{=} \frac{p_{m}}{p_{n}}, \\
& U_{m n} \stackrel{d}{=} \frac{u_{m}}{a_{n}}=\frac{u_{m}}{\sqrt{(\gamma R T)_{n}}},
\end{aligned}
$$

and

$$
A_{m n} \stackrel{d}{=} \frac{a_{m}}{a_{n}}=\sqrt{\frac{(\gamma R T)_{m}}{(\gamma R T)_{n}}}
$$


where $m$ and $n$ would normally represent 1 and 4 , respectively. It is necessary to determine the new pressure established in region 2 just behind the shock, and for this a Jacobien iteration scheme works best according to the following formula:

$$
P_{21, i+1}=1-\left[\left(P_{14} P_{21, i}\right)^{\beta_{4}}-1\right] \sqrt{\frac{\left(\alpha_{1} P_{21, i}+1\right)}{\beta_{4} E_{14}}}
$$

Once $\mathrm{P}_{21}$ converges, usually to within $0.01 \%$ relative error after about 20 iterations, Glass' method continues to solve for

1) Density Ratios:

$$
\begin{aligned}
& \frac{\rho_{3}}{\rho_{4}}=\Gamma_{34}=\left(P_{14} P_{21}\right)^{1 / \gamma_{4}} \\
& \frac{\rho_{2}}{\rho_{1}}=\Gamma_{21}=\frac{1+\alpha_{1} P_{21}}{\alpha_{1}+P_{21}}
\end{aligned}
$$

2) Sonic Speed and Temperature Ratios

$$
\begin{aligned}
& \frac{a_{3}}{a_{4}}=A_{34}=\sqrt{\frac{(\gamma R T)_{3}}{(\gamma R T)_{4}}}=\sqrt{T_{34}}=P_{34}^{\beta_{4}}=\left[P_{14} P_{21}\right]^{\beta_{4}} \\
& \frac{a_{2}}{a_{1}}=A_{21}=\sqrt{\frac{(\gamma R T)_{2}}{(\gamma R T)_{1}}}=\sqrt{T_{21}}=\left[\frac{P_{21}\left(\alpha_{1}+P_{21}\right)}{1+\alpha_{1} P_{21}}\right]^{1 / 2}
\end{aligned}
$$

3) Shock Wave Mach Number and Velocity

$$
M_{S}=\frac{w_{1}}{a_{1}}=W_{11}=\sqrt{\beta_{1}\left(1+\alpha_{1} P_{21}\right)}
$$

4) Contact Front Velocity 


$$
\begin{gathered}
\frac{u_{2}}{a_{1}}=U_{21}=\frac{\left(P_{21}-1\right)}{\gamma_{1} \sqrt{\beta_{1}\left(\alpha_{1} P_{21}+1\right)}} \\
\frac{u_{3}}{a_{4}}=U_{34}=\frac{a_{1}}{a_{4}} \frac{u_{3}}{a_{1}}=\frac{a_{1}}{a_{4}} \frac{u_{2}}{a_{1}}=A_{14} U_{21}
\end{gathered}
$$

5) Head and Tail Rarefaction Velocities

$$
\begin{gathered}
\frac{C_{4}^{-}}{a_{4}}=c_{44}^{-}=\frac{u_{4}-a_{4}}{a_{4}}=-1 \\
\frac{C_{3}^{-}}{a_{4}}=c_{34}^{-}=\frac{1}{\beta_{4} \gamma_{4}}\left[1-\left(P_{14} P_{21}\right)^{\beta_{4}}\right]-\left(P_{14} P_{21}\right)^{\beta_{4}}
\end{gathered}
$$

6) Local Mach Numbers

$$
\begin{aligned}
& M_{3}=\frac{u_{3}}{a_{3}}=\frac{u_{3}}{a_{4}} \frac{a_{4}}{a_{3}}=\frac{U_{34}}{A_{34}} \\
& M_{2}=\frac{u_{2}}{a_{2}}=\frac{u_{2}}{a_{1}} \frac{a_{1}}{a_{2}}=\frac{U_{21}}{A_{21}}
\end{aligned}
$$

The next significant event in the shock tube occurs when the shock wave is reflected at the closed end of the tube, as represented in Figure 13.

$\left(t=t_{S 1, R F L}\right)$

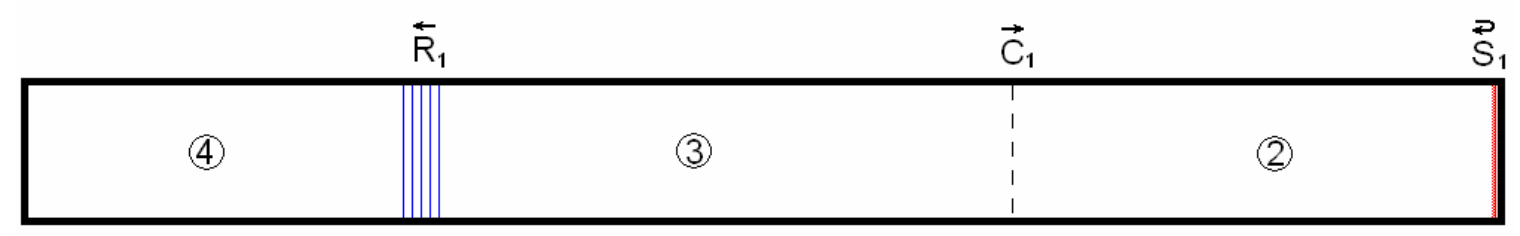

Figure 13: The first shock $\left(S_{1}\right)$ is reflected at the end of the closed tube. 
It is at the tube end that the velocity of fluid moving in region 2 must necessarily be brought to rest to maintain zero wall velocity after the reflected shock moves back into region $2\left(u_{5}=0\right)$ and the pressure ratio can be solved according to

$$
\frac{p_{5}}{p_{2}}=P_{52}=\frac{\alpha_{1}+2-P_{12}}{1+\alpha_{1} P_{12}} .
$$

Similarly, the density and temperature ratios are found as

$$
\frac{\rho_{5}}{\rho_{2}}=\Gamma_{52}=\frac{1+\alpha_{1} P_{52}}{\alpha_{1}+P_{52}}
$$

and

$$
\frac{T_{5}}{T_{2}}=T_{52}=\frac{P_{52}\left(\alpha_{1}+P_{52}\right)}{1+\alpha_{1} P_{52}} .
$$

The new reflected Mach wave number is found as

$$
M_{S, 2}=W_{22}=M_{S}\left[\frac{2+\left(\alpha_{1}-1\right) P_{12}}{\alpha_{1}+P_{12}}\right]
$$

where it is worth noting that in the event of a weak initial shock, or $\mathrm{P}_{12} \approx 1, \mathrm{M}_{\mathrm{S}, 2}$ and $\mathrm{M}_{\mathrm{S}}$ are essentially sonic Mach waves. Finally, the time required to reach this condition is easily calculated as

$$
t_{S 1, R F L}=\frac{L_{1}}{M_{S} a_{1}} .
$$

The time required to allow the starting shock to clear the initial length of the tube, $\mathrm{t}_{\mathrm{S} 1, \mathrm{RFL}}$, has been successfully used in literature as one of the characteristic times governing real fluid systems that have been modeled numerically $[31,35]$. 
Referring to Figure 11, if $\mathrm{L}_{4}$ is sufficiently short in comparison to $\mathrm{L}_{1}$, the next significant event in the shock tube will be the reflection of the rarefaction wave at the end of the tube in region 4 . If on the other hand $L_{1}$ and $L_{4}$ are nearly equal, the next event will be the refraction of the reflected shock at the contact front preceding region 3 . This case is shown in Figure 14.

$\left(t=t_{\text {C1-S2, RFR }}\right)$

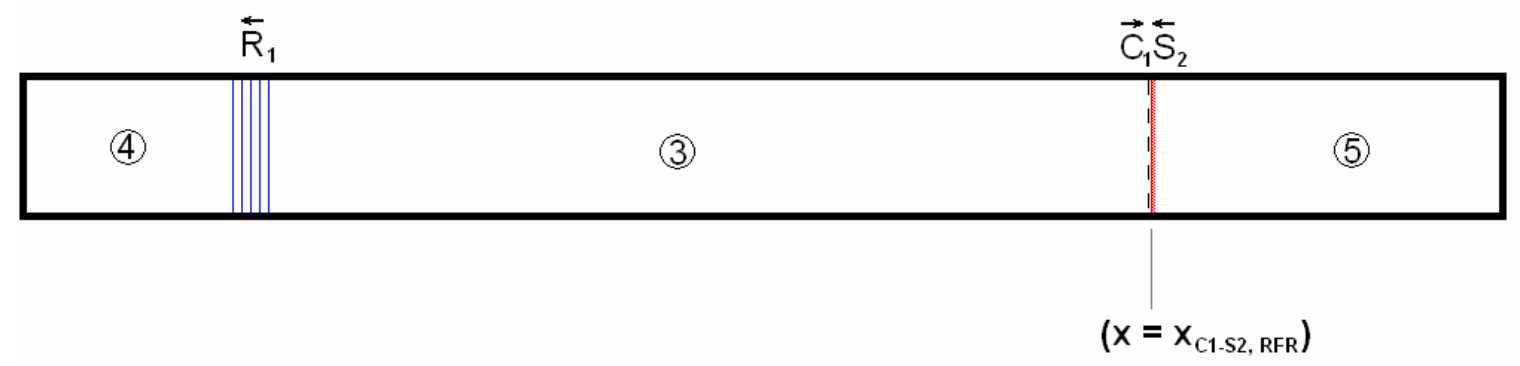

Figure 14: The first head-on collision in a tube of equal region 1 and region 4 initial lengths will be between the reflected shock and the contact front.

The location of the collision can be determined as

$$
x_{C 1-S 2, R F R}=L_{1}\left[\frac{1}{M_{S} a_{1}}+\frac{1}{M_{S, 2} a_{2}}\right]\left[\frac{1}{u_{2}}+\frac{1}{M_{S, 2} a_{2}}\right]^{-1}
$$

so that the time to collision is then solved as

$$
t_{C 1-S 2, R F R}=\frac{x_{C 1-S 2, R F R}}{u_{2}} .
$$

Immediately following the refraction, three possibilities can occur depending upon the values of $\mathrm{P}_{45}$ and $\mathrm{E}_{15}$ : reflection of a shock wave, Mach wave, or rarefaction wave. Again from Glass, the case for a reflected shock occurs when

$$
E_{15}<\frac{\alpha_{1}+P_{54}}{\alpha_{5}+P_{54}}
$$


where

$$
\begin{gathered}
E_{15}=\frac{\alpha_{1}+P_{54} P_{43} h}{\left(1-P_{54} P_{43}\right)^{2}}\left[k \sqrt{P_{43}}-\frac{P_{43}-1}{\sqrt{\alpha_{1}+P_{43}}}\right]^{2}, \\
h=\frac{\left(\alpha_{5} P_{54}+1\right)}{\left(\alpha_{5}+P_{54}\right)},
\end{gathered}
$$

and

$$
k=\frac{\left(1-P_{54}\right)}{\sqrt{\alpha_{5} P_{54}+1}} .
$$

The case for a reflected Mach wave occurs when

$$
E_{15}=\frac{\alpha_{1}+P_{54}}{\alpha_{5}+P_{54}}
$$

Finally, the case for the reflected rarefaction wave occurs when

$$
E_{15}>\frac{\alpha_{1}+P_{54}}{\alpha_{5}+P_{54}}
$$

noting that the pressure ratio between regions 5 and 4 is solved as

$$
P_{54}=P_{52} P_{21} P_{14}
$$

It can be shown that for the case of a "cold" shock tube beginning at a relatively low value of $\mathrm{P}_{41}(<10)$ that the case of the reflected rarefaction wave is the only case which can occur as the moving shock wave meets the contact surface. The strength of the shock $\mathrm{S}_{2}$ is reduced, and the "new" shock is renamed $\mathrm{S}_{3}$ (Figure 15). The behavior of the new rarefaction zone, $\mathrm{R}_{2}$, and the thermodynamic relationship between the newly formed region 7 and region 5 can be determined using the same method as outlined for regions 3 and 4 (Figure 12). Likewise, the relationship between regions 8 and 3 can be determined using the method as outlined for regions 1 and 2. 


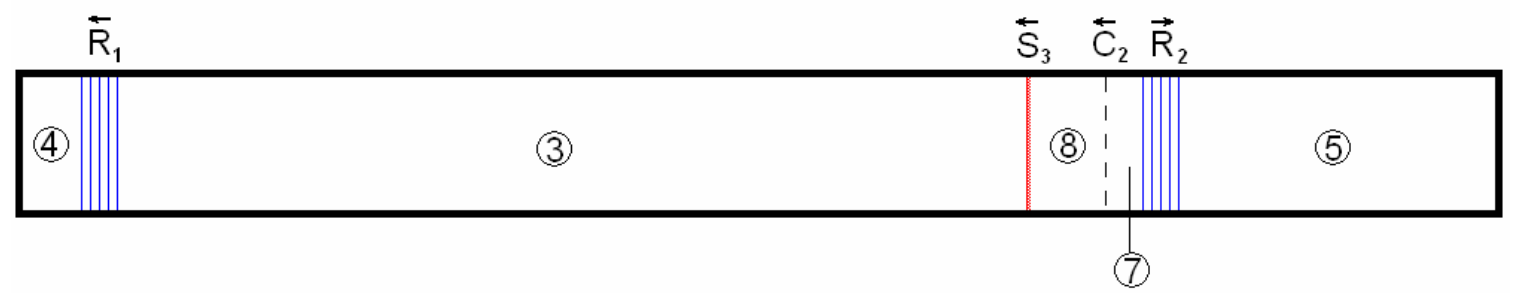

Figure 15: The head-on collision gives rise to a second rarefaction wave $\left(\mathbf{R}_{2}\right)$.

The next significant event in the shock tube wave system occurs when the first rarefaction zone, $\mathrm{R}_{1}$, contacts the other closed end of the tube and is reflected back into the moving particle region 3 as shown in Figure 16. As was the boundary condition of the reflected shock wave, the particles behind the reflected tail rarefaction wave which make up the new region 6 must come to rest $\left(\mathrm{u}_{6}=0\right)$. The conditions within region 6 are given from Glass as

$$
\begin{gathered}
P_{64}=\left[2 P_{34}^{\beta_{4}}-1\right]^{1 / \beta_{4}}, \\
T_{64}=\left[2 P_{34}^{\beta_{4}}-1\right]^{2},
\end{gathered}
$$

and

$$
\Gamma_{64}=\left[2 P_{34}^{\beta_{4}}-1\right]^{1 / \gamma_{4} \beta_{4}},
$$

noting that

$$
P_{34}=\frac{p_{3}}{p_{1}} \frac{p_{1}}{p_{4}}=\frac{p_{2}}{p_{1}} \frac{p_{1}}{p_{4}}=P_{21} P_{14} .
$$


$\left(t=t_{R 1, R F L}\right)$

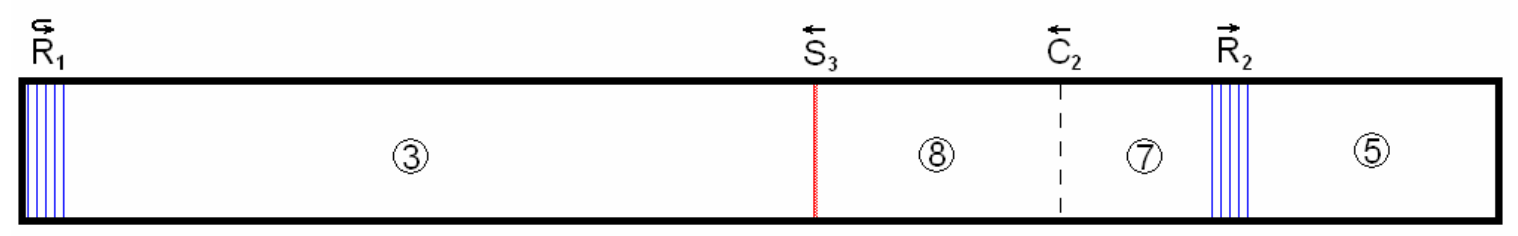

Figure 16: The first rarefaction zone $\left(R_{1}\right)$ is reflected at the end of the closed tube.

The time required for region 6 to reach steady conditions is another key time used in literature to develop experimental and numerical models [31,35]. According to Glass, the dimensionless time constant of interest with regard to the formation of a steady region 6 for a diatomic gas is found as

$$
\tau_{6}=\frac{a_{4} t_{R I, R F L}}{L_{4}}=\frac{1}{\left(2 P_{34}^{1 / 7}-1\right)^{3}}+\frac{6 P_{34}^{2 / 7}\left(1-P_{34}^{1 / 7}\right)^{2}}{\left(2 P_{34}^{1 / 7}-1\right)^{5}}
$$

Glass also offers an example showing that for $\mathrm{P}_{41}=10.5, \mathrm{~L}=1 \mathrm{ft} ., \mathrm{T}_{1}=72^{\circ} \mathrm{F}, \mathrm{t}_{\mathrm{R} 1, \mathrm{RFL}}=$ 3.82 milliseconds. As the initial pressure ratio is increased, more relative mass is added to the initial region 4 and it requires more time for the starting rarefaction wave to reach the end of the tube. This result is illustrated by a second example provided by Glass in which for $\mathrm{P}_{41}=100, \mathrm{~L}=1 \mathrm{ft} ., \mathrm{T}_{1}=72^{\circ} \mathrm{F}$. Rarefaction clearance time of $\mathrm{t}_{\mathrm{R} 1, \mathrm{RFL}}=71.1$ milliseconds.

The shock wave $S_{3}$ and the newly reflected rarefaction zone $R_{3}$ must meet each other in a head-on collision. This situation is depicted in Figure 17, and the resulting strengthened shock wave $S_{4}$, weakened rarefaction zone $R_{4}$, and contact zone $C_{3}$ are displayed with their respective directions in Figure 18. An interesting impact of the shock wave penetration of the rarefaction zone is that the strength of the shock $\mathrm{S}_{3}$ 
continuously increases as it moves from the head to the tail of the rarefaction zone $\mathrm{R}_{3}$ and thus the entropy rise also continuously increases during the process leaving in its wake the first contact zone $\mathrm{C}_{3}$.

$$
\left(t=t_{R 3-S 3, C O L}\right)
$$

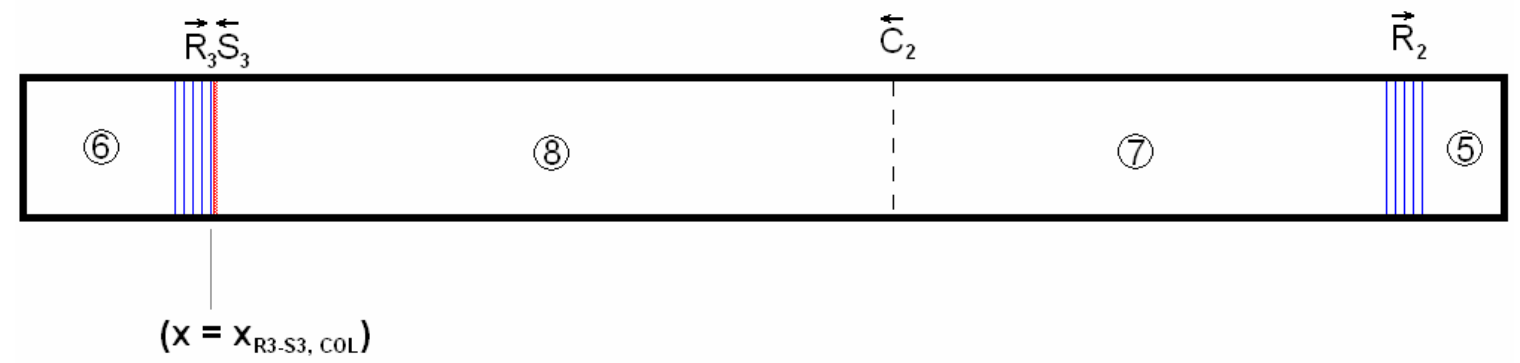

Figure 17: The head-on collision of the reflected rarefaction wave $\left(R_{3}\right)$ and the refracted shock wave $\left(\mathbf{S}_{3}\right)$.

$$
\left(t=t_{R 2, R F L}\right)
$$

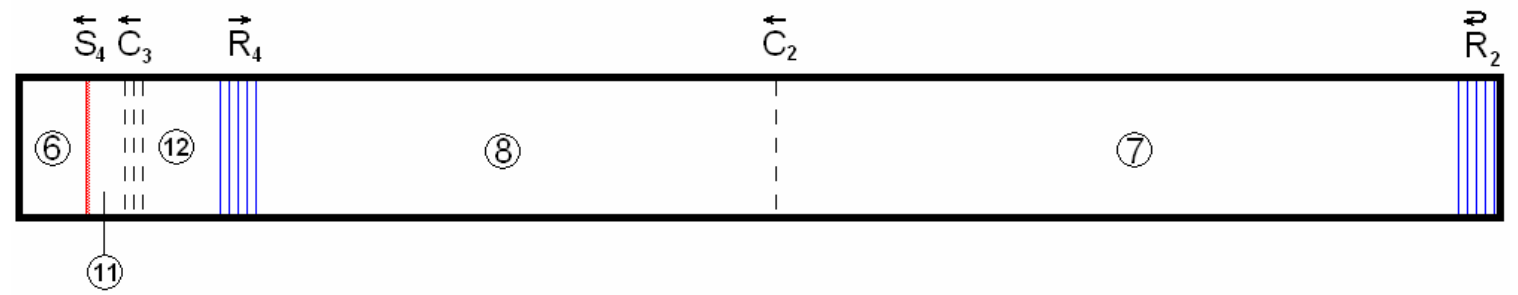

Figure 18: The second rarefaction wave $\left(R_{2}\right)$ reflection and the contact zone $\left(C_{3}\right)$ created from the head-on collision of Figure 17.

The pressures within the newly formed regions 11 and 12 may be determined, again from Glass, starting with an iterative solution of

$$
P_{11-6}=1+\sqrt{1+\alpha_{1} P_{11-6}}\left\{D-E\left[\left(P_{11-6} P_{38}\right)^{\frac{1}{\alpha_{1}+1}}-\left(P_{86}\right)^{\frac{1}{\alpha_{1}+1}}\right]\right\},
$$

where 


$$
D=\left(P_{86}\right)^{\frac{1}{\alpha_{1}+1}} \frac{P_{83}-1}{\sqrt{1+\alpha_{1} P_{83}}}+\sqrt{\alpha_{1}+1}\left[1-\left(P_{86}\right)^{\frac{1}{\alpha_{1}+1}}\right]
$$

and

$$
E=\sqrt{\left(\alpha_{1}+1\right) P_{83}\left[\left(\alpha_{1}+P_{83}\right) /\left(1+\alpha_{1} P_{83}\right)\right]} .
$$

Next, by recognizing the necessary condition that pressures remain constant across the contact zone, it is possible to solve for $\mathrm{P}_{12-8}$ such that

$$
P_{12-8}=P_{11-6} P_{63} P_{38}
$$

The next five figures demonstrate exactly how complicated the "simple" wave system within the shock tube can become within a fraction of a second. In Figure 19, the strengthened shock $\mathrm{S}_{4}$ reaches the end of the tube and is reflected back into region 11 . Figure 20 shows the reflected shock $\mathrm{S}_{5}$ at the instant of head-on collision with the contact zone $\mathrm{C}_{3}$. Again, this type of collision results in the refraction of the shock wave, the creation of a new contact front, and the reflection of a new rarefaction wave (Figure 21). Figure 22 shows the head-on collision of the rarefaction wave $\mathrm{R}_{4}$ with the contact surface $\mathrm{C}_{2}$ just after reflection of rarefaction wave $\mathrm{R}_{6}$. This type of collision results in the refraction of the contact surface $C_{2}$ becoming $C_{5}$, the refraction of rarefaction zone $R_{4}$ into $\mathrm{R}_{8}$ and the reflection of $\mathrm{R}_{4}$ into $\mathrm{R}_{9}$ (Figure 23 ). 


\begin{tabular}{|c|c|c|c|c|c|}
\hline$\overleftarrow{S}_{4} \overleftarrow{C}_{3}$ & $\vec{R}_{4}$ & & & & $\xi_{5}$ \\
\hline (11) III & & (8) & & (7) & (13) \\
\hline
\end{tabular}

Figure 19: A shock wave $\left(S_{4}\right)$ is reflected from the end of the tube originally containing the high pressure gas.

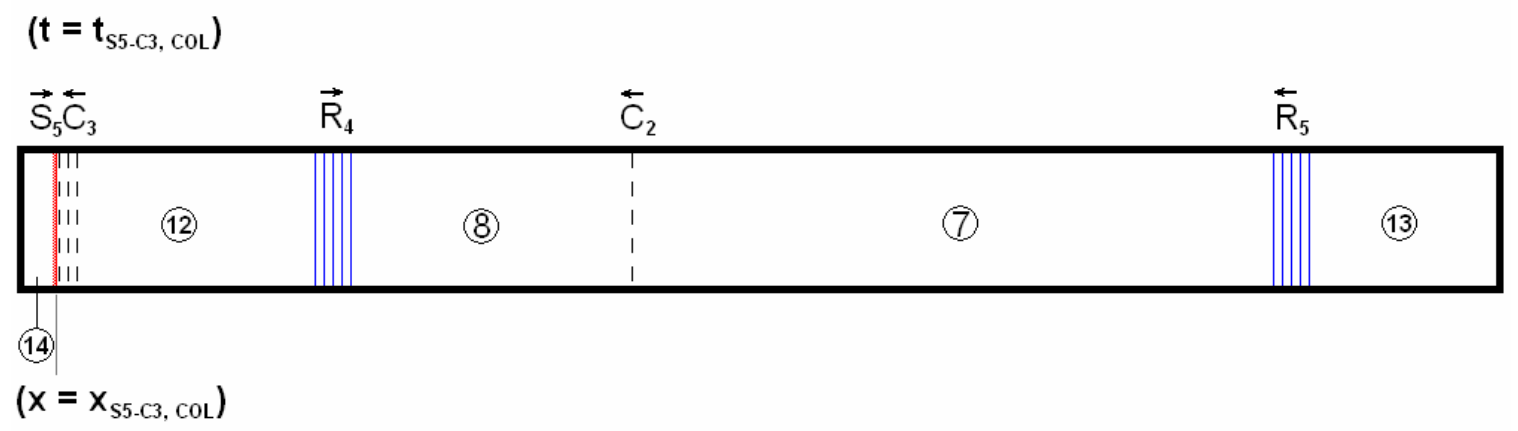

Figure 20: The reflected shock $\left(S_{5}\right)$ enters the spread out contact zone $\left(C_{3}\right)$ head-on.

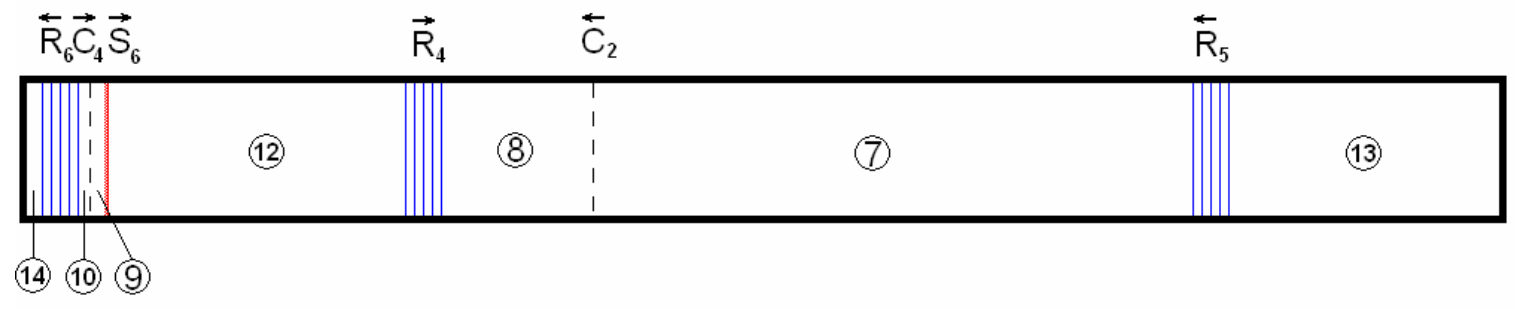

Figure 21: Another rarefaction zone $\left(R_{6}\right)$ is created as the refracted shock passes through the reformed contact zone $\left(\mathrm{C}_{4}\right)$. 


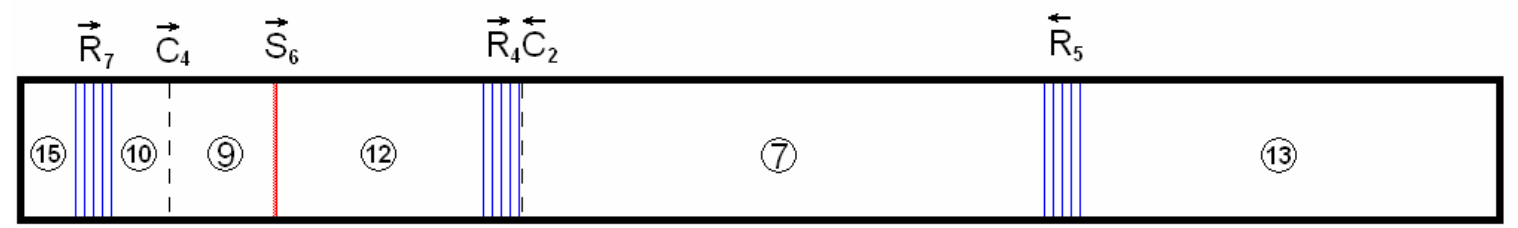

Figure 22: After the reflection of the rarefaction wave $\left(\mathbf{R}_{7}\right)$, an older rarefaction zone $\left(\mathbf{R}_{4}\right)$ collides head-on with a contact front $\left(\mathrm{C}_{2}\right)$.

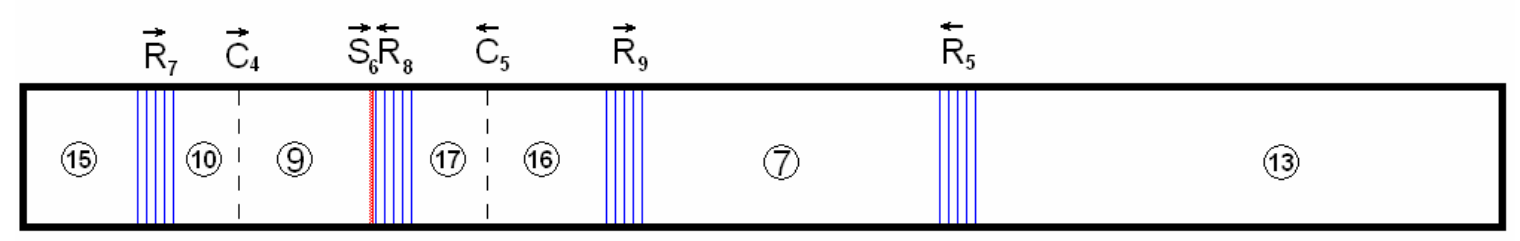

Figure 23: Refraction $\left(\mathbf{R}_{9}\right)$ and reflection $\left(\mathbf{R}_{8}\right)$ of rarefaction zones after the collision of Figure 22.

Depending upon the length of the shock tube and its initial pressure ratio, the wave system started within the tube can easily give rise to as many as 17 thermodynamically unique regions within less than 1 second. It is also important to understand that during all of the shock wave movement, entropy within the system is on the rise, eventually weakening the entire wave system to a point of complete attenuation of shock waves. Generally speaking, for low initial pressure ratio systems of short overall length (such as the cylinder-CCV-cylinder model used in this research), shock wave attenuation happens fast enough that the starting shock $\left(\mathrm{S}_{1}\right)$ loss dominates all other entropy generating transient behavior. In such cases, as suggested in the literature by Arkhipov et al. [31], only the longest of either $t_{\mathrm{R} 1, \mathrm{RFL}}$ or $\mathrm{t}_{\mathrm{S} 1, \mathrm{RFL}}$ is used to establish the final time for an exponential flow rate increase from zero up to the quasi-steady flow rate 
which has been measured or adjusted from theory by some experimentally determined discharge coefficient. 


\section{Chapter 5: Experimental Apparatus and Procedure}

\section{Constant Pressure Experiments}

\section{General Arrangement}

Two basic experimental procedures were used to collect the main body of data. In the first set of experiments, a constant pressure process was investigated in which the $\mathrm{CCV}$ was used to discharge an "infinite" volume source held at relatively high pressure into ambient surroundings considered to be at relatively low pressure. The setup for these experiments is shown schematically in Figure 24.

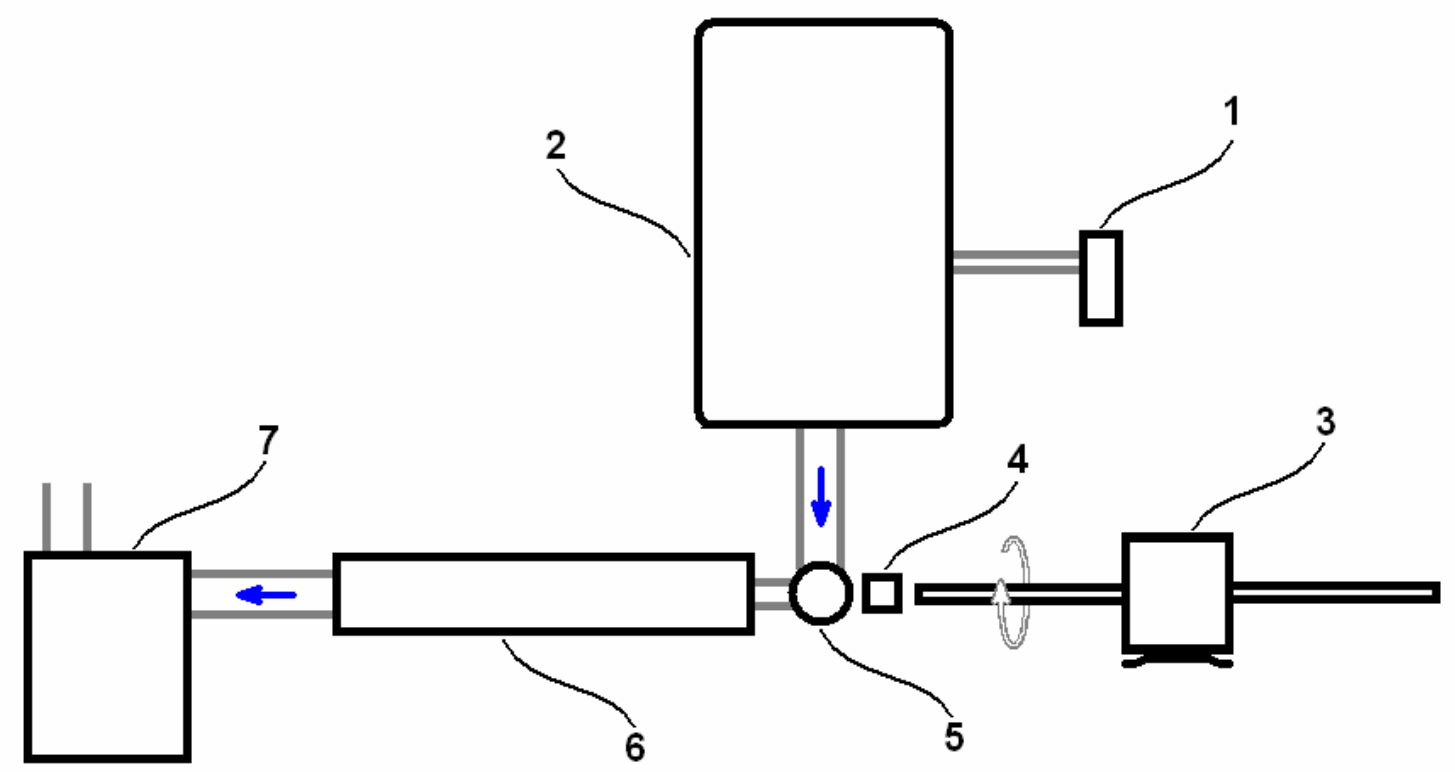

Figure 24: The constant pressure experimental setup.

Referring to Figure 24, air was first compressed and partially contained in a first stage holding volume of 10 gallons $(37.9 \mathrm{~L})$ which was connected to a larger 12 gallons (45.4 L) second stage holding volume (2) through a pressure regulator (1). Freshly 
compressed air was allowed at least two hours to come to thermal equilibrium with ambient temperature before proceeding to turn on the 5 speed CCV motor (3) which was set to the desired speed and allowed a short amount of time to come to constant speed. Upon initializing the data acquisition (DAQ) software, a brief excitation signal was introduced to engage the electromechanical clutch (4) so that the CCV (5) would suddenly accelerate to the $\mathrm{CCV}$ motor speed and rotate for a single revolution before coming to rest. Depending upon the motor speed, the duration of time that the CCV remained open was on the order of $\sim 15$ milliseconds. The burst of pressurized gas moved rapidly through the shock tube assembly (6) and was then exhausted into the mass collection chamber (7).

\section{Apparatus}

The drive used to turn the CCV was powered by an A.O. Smith 5 speed AC motor (Figure 25). The unit was mounted sufficiently high enough on a base stand to allow easy connection to the rest of the CCV assembly at the drive end of the shaft and also allow fan blade clearance at the free end of the motor shaft. A rotary switch (1) was used to select the drive speed, which was electronically read from two circuits (2). A magnetic pick up switch indicated a single pulse per revolution, and a CNC-milled disk provided 24 pulses per revolution ( 1 pulse per $\left.15^{\circ}\right)$. The drive was positioned so that a Lovejoy (jaw) type coupling (3) meshed with an electromechanical clutch receiver (not shown). The AC motor required some amount of preload torque to be applied to the system in order to achieve the operational range of speeds. Preload was applied using both a static load system (4) and a dynamic load system (5). Constant load torque could be applied using the static system by way of adding washers to a bucket mounted above a freely 
rotating boom that pressed a rubber brake pad against the wide edge of the flywheel. A pin inside the bucket ensured that the washers were always held at a fixed distance from the pivoting elbow, thus allowing precise control over this particular load source from one test set to another. Additional load torque was applied by way of the attached fan blade assembly. This measure helped to provide a more automated preload system that would increase load torque with an increase in motor speed.

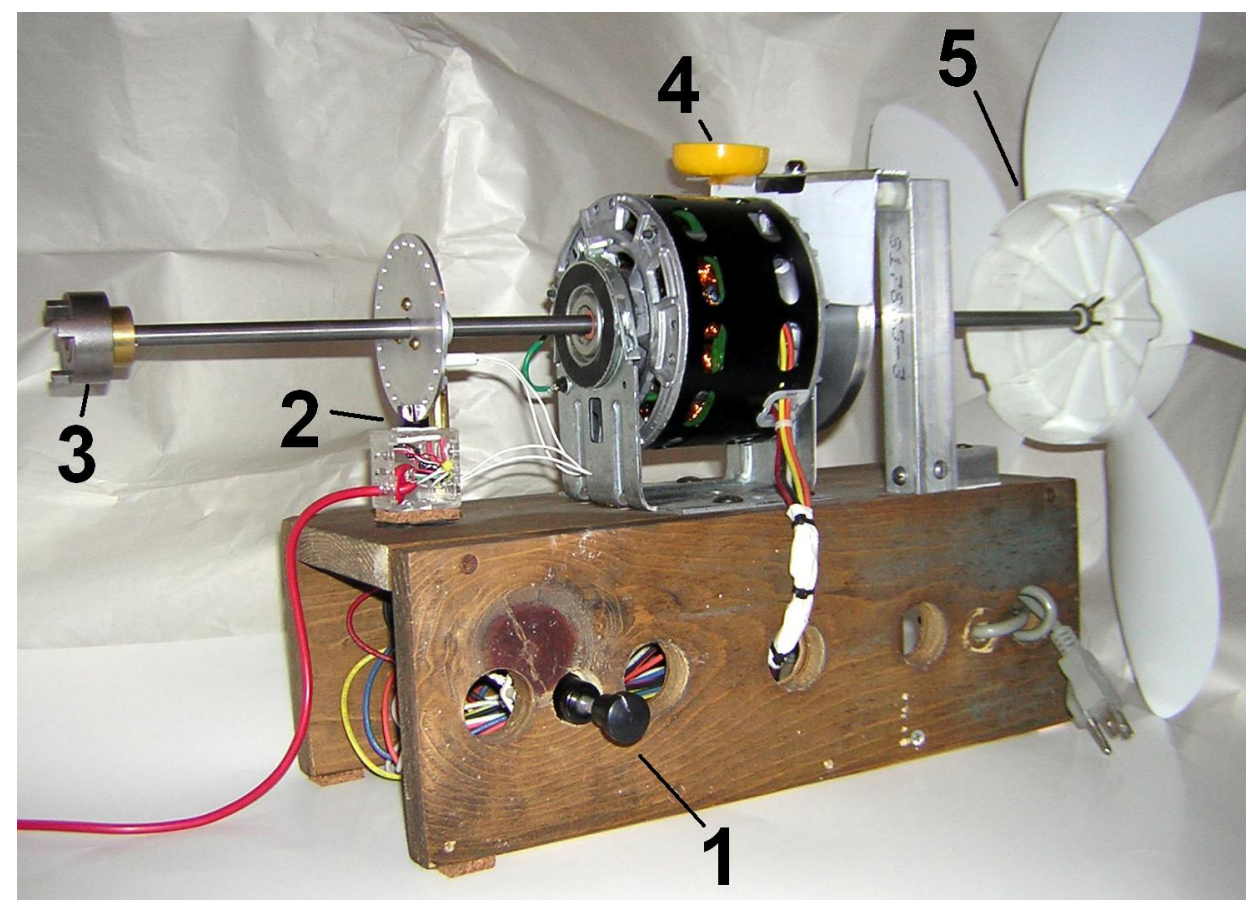

Figure 25: The 5 speed CCV motor was used to supply the constant RPM CCV shaft input.

The CCV assembly itself was made of a number of specialized parts. The commercially made electromechanical clutch (EC75LL by Reell MFG) provided electronically controlled engagement of the $\mathrm{CCV}$ input shaft to the CCV motor. Important specifications for the clutch included a zero-to-full speed time of less than 3 milliseconds, a maximum input torque of up to $75 \mathrm{ft}-\mathrm{lb}_{\mathrm{f}}$, a maximum operating speed of 
up to 1400 RPM, and a nominal excitation voltage of 24 VDC. The clutch is shown in Figure 26 to the far right (1) where just outside of the picture the mated jaw type coupling from the CCV motor provided available constant speed input. An input shaft adapter (2) was machined to properly mate the freewheeling side of the clutch to the control stem of the CCV ball. The ridges of this hexagonally shaped adapter were utilized as a means of providing a variable depth motion in front of the infrared (IR) CCV position sensor. Any time that the clutch was engaged, the adapter would begin to turn resulting in an apparent sinusoidal motion directly in front of the IR range sensor. Thus, the corresponding output for the CCV position sensor showed a 6 peak sinusoid for a single full rotation of the CCV. The main body of the CCV (4) housed the specially modified $3 / 4$ " ball used in all the experiments.

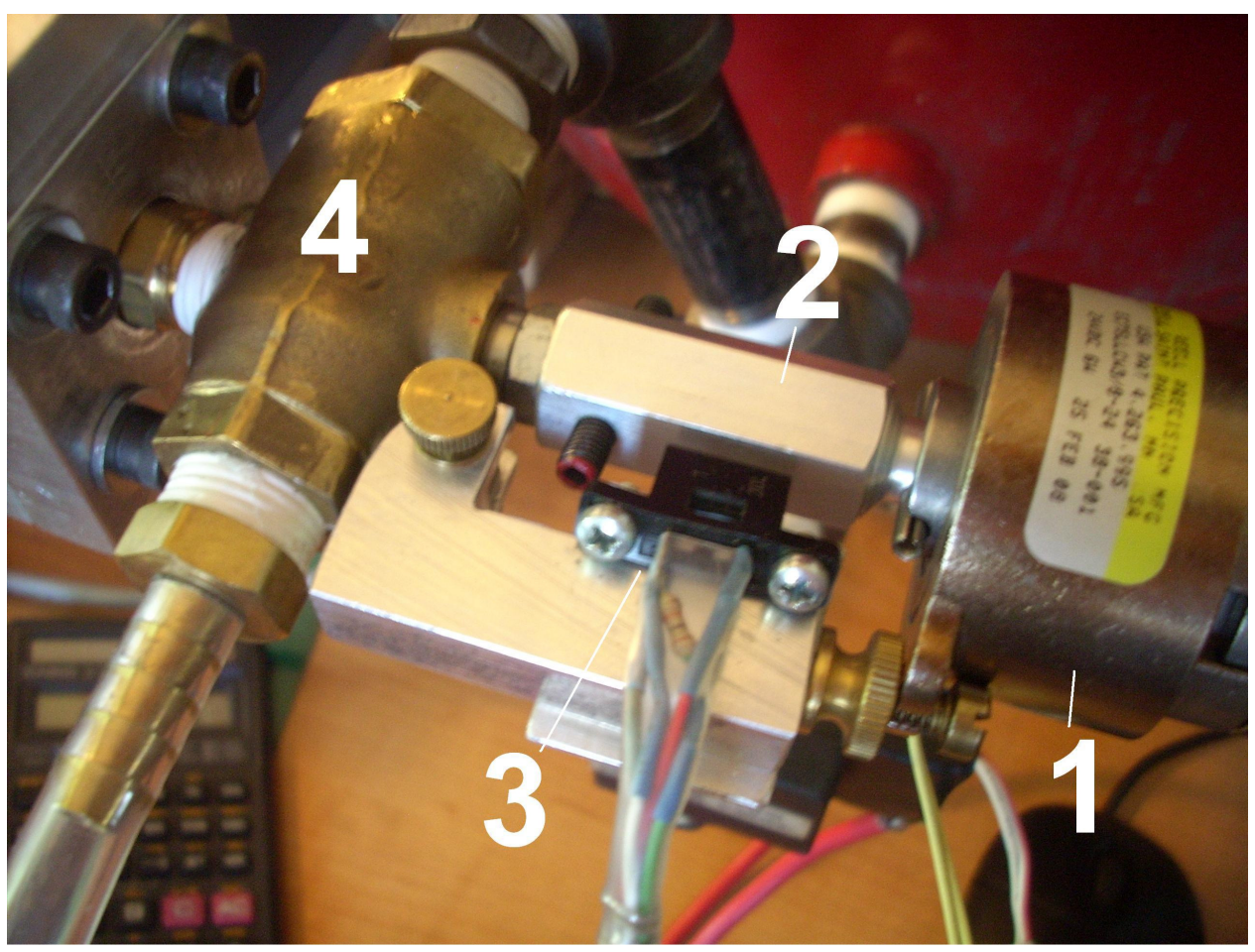

Figure 26: The CCV assembly consisted mainly of an input clutch (1), input shaft adapter (2), CCV position sensor (3) and main ball housing (4). 
The CCV itself was fabricated using an existing commercially available off-theshelf ball type valve. Modifications to the valve involved replacement of the stock central ball which had a straight hole of 0.371 " diameter with a new angled hole of 0.375 " diameter (Figure 27). In order to facilitate the use of the new ball hole design, a 1/4" NPT female threaded hole was made on the side of the original ball valve housing. A CAD model cross sectional view of this design is shown in Figure 28. The stock exhaust hole was ultimately used as a bleed valve hole which could be opened to quickly reestablish pressure equilibrium between the mass collection chamber and ambient air after a test had been run.

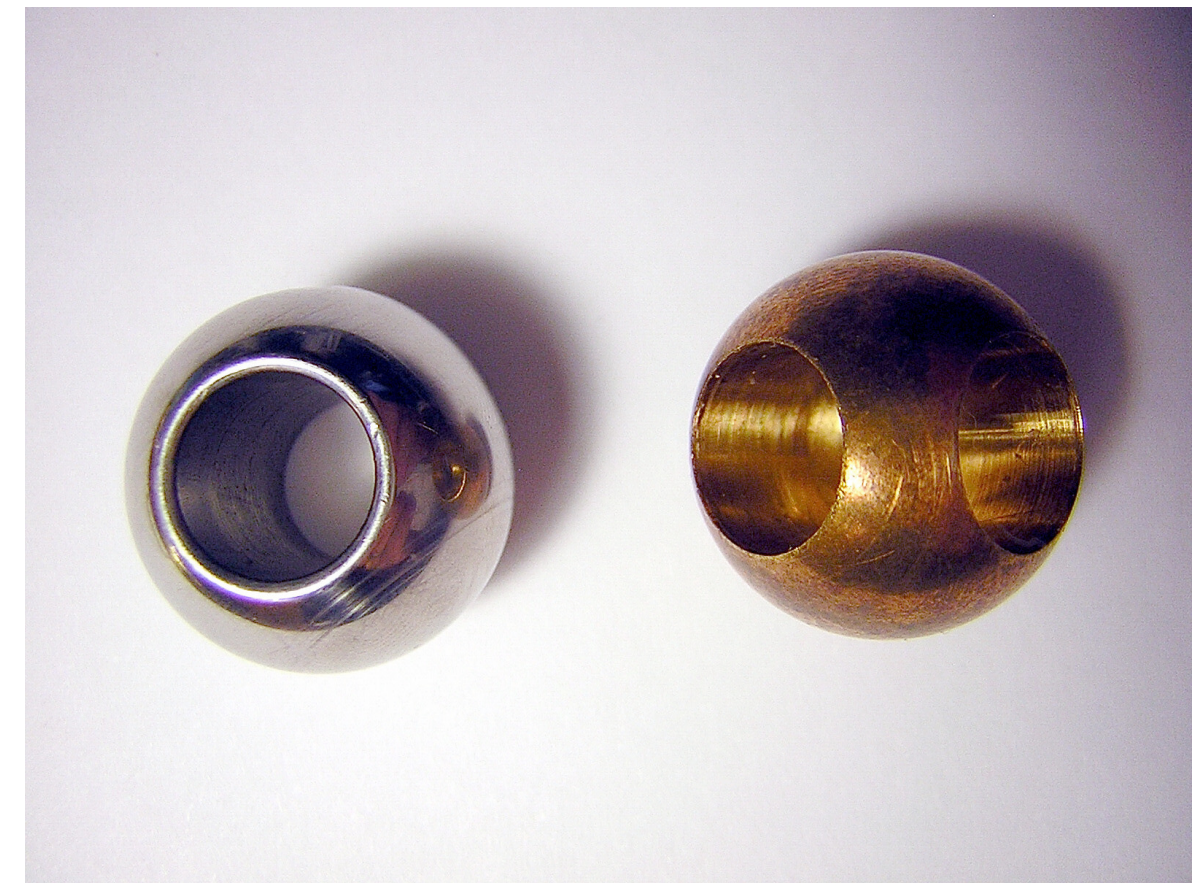

Figure 27: The original ball (left) which had a straight 0.371 " hole was replaced with a new ball (right) which had a $90^{\circ} 0.375 "$ hole. 


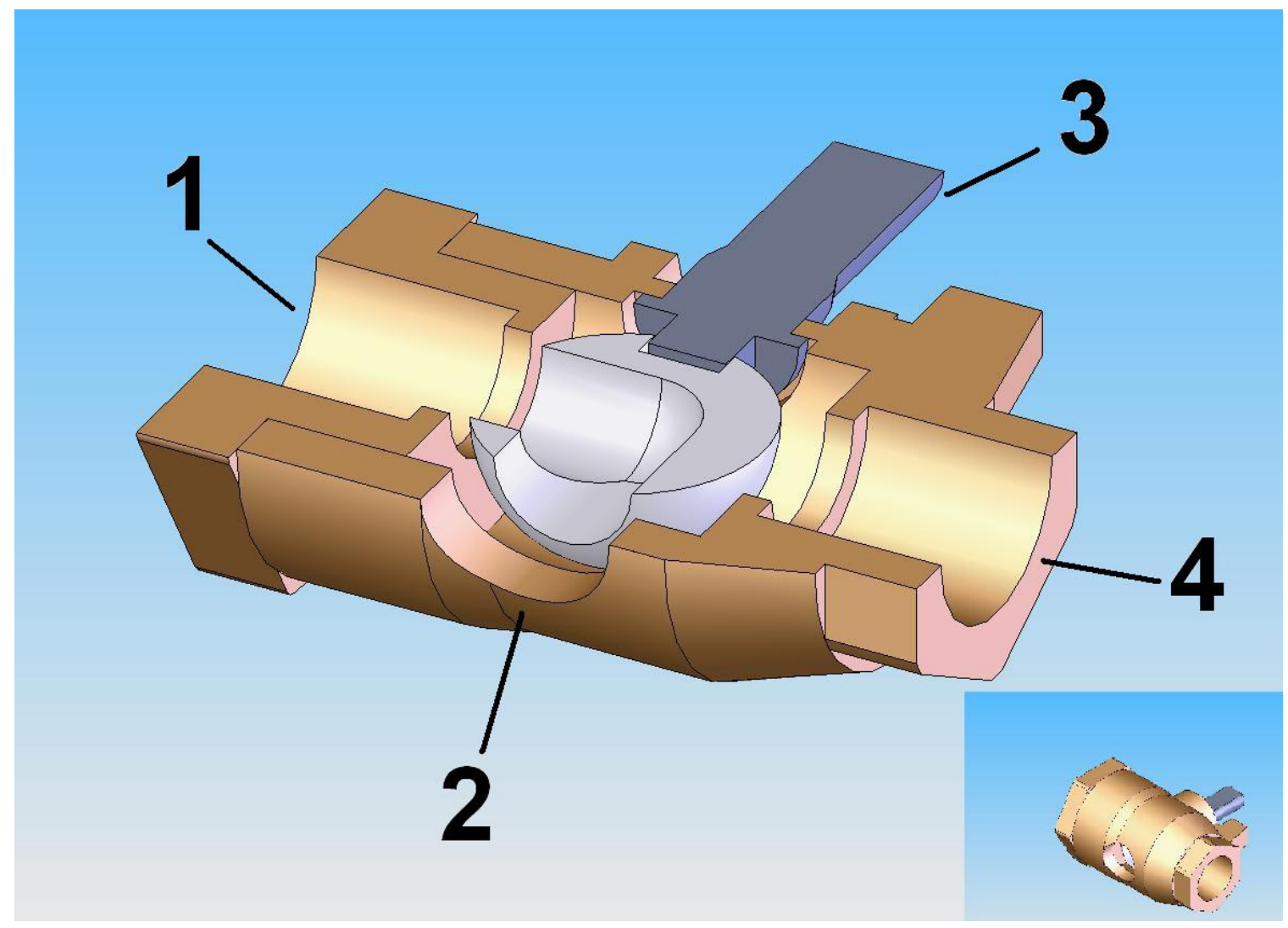

Figure 28: A cutaway view of the CCV design showing the inlet (1), modified exhaust location (2), CCV control stem (3), and pressure relief exhaust location (4).

Plumbing on the entire constant pressure experimental setup was carefully planned so that no section before or after the $\mathrm{CCV}$ offered an opportunity for the moving fluid to choke. The minimum area was in fact controlled by a replaceable set of varying diameter nozzles mounted between the CCV and the shock tube assembly (Figure 29). Inlets for the choke nozzles were filleted with a $3 / 4 "$ ball-end mill to allow the nozzle to come extremely close to the $3 / 4 " \mathrm{CCV}$ ball. The outlet of the nozzles expanded at a $7^{\circ}$ angle from their minimum diameters to 0.375 ", thus matching the geometry of the shock tube before the gas left the nozzle. 


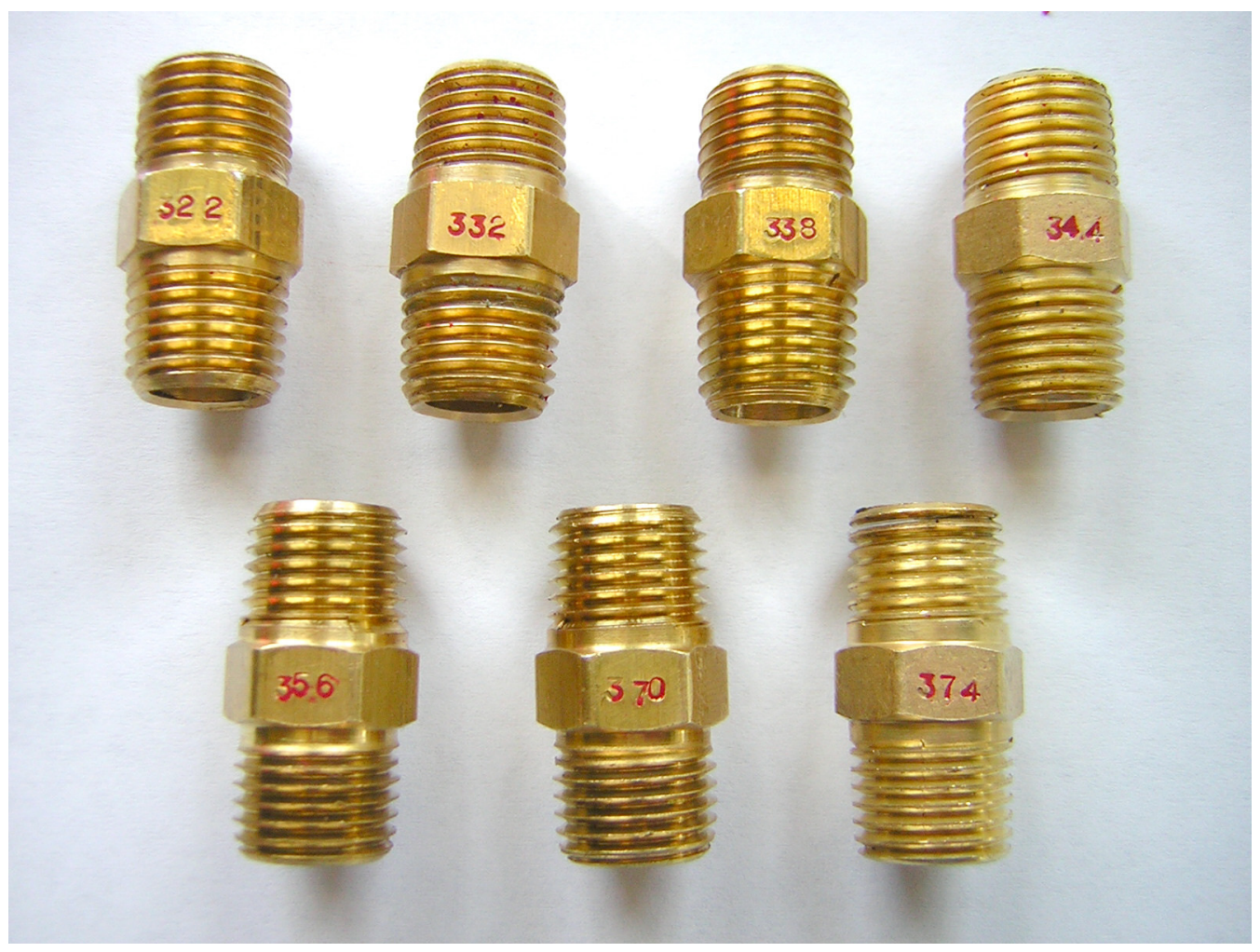

Figure 29: Nozzles of various diameters were used to force the flow to choke at the exit of the CCV (diameters in thousandths of an inch are stamped on the nozzle bodies).

The $13 " \times 2 " x 2.25 "$ shock tube assembly was the most carefully considered piece of sensor equipment fabricated. The basic function of the tube was to measure high frequency pressure trends at a response rate of up to $22 \mathrm{kHz}$ within the tube to better understand the transient exhaust behavior of the CCV. In a qualitative sense, the capacitive discharge microphone sensors mounted along the tube walls were able to indicate basic pressure related fluid behavior within the tube as well as frame vibration behavior from the tube walls. In a quantitative sense, the sensors were extremely useful in determining the starting shock Mach number of each test.

The assembly itself consisted of 5 major parts: a top thread plate, two end flanges, an upper channel half, and a lower channel half. Eight recessed holes were machined at 
regular intervals of 1.500 " along the length of the upper channel half with corresponding plug threads on the top thread plate. The upper and lower channel halves were machined separately to allow easy access to the channel along its entire length. Once the two halves were nestled together and sealed using silicone sealant, the two end flanges were mounted to allow the threaded attachment of the choke nozzle at the inlet end and an outlet hose barb at the exhaust end. The top thread plate allowed the air tight attachment of microphone subassemblies or solid plugs to the recessed holes. A small PVC block mounted to the upper channel half (Figure 30) served as a terminal strip for sensor wiring and also allowed the entire assembly to be grounded by one of the two mounting bolts so that when the sensors were placed into their respective holes, the contact between the bases of the holes and the aluminum microphone casings formed a ground, thus eliminating the need for additional wires.

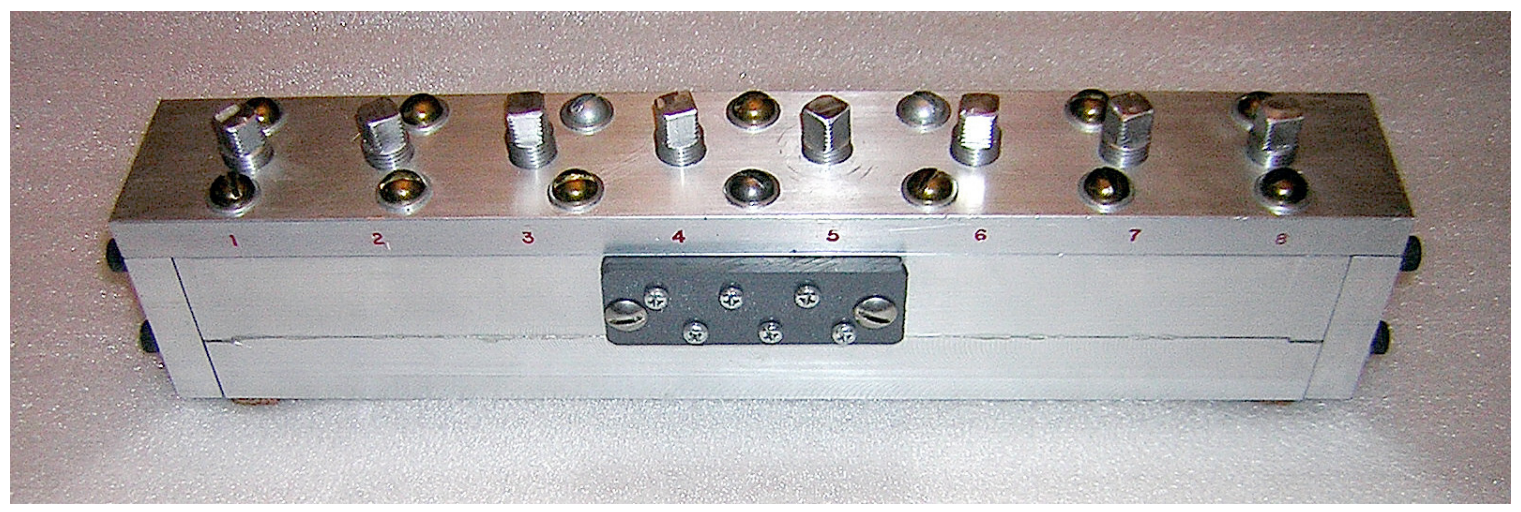

Figure 30: The shock tube assembly was capable of holding up to eight microphone sensors spaced along the tube wall in 1.500" increments (fluid moved from left to right as pictured).

Microphone sensors were attached to the shock tube using a variety of specially machined hardware. Referring to Figure 31, if a sensor was used in one of the receiver holes, the sensor unit (1) was first placed head down into the hole, then sealed and 
secured in place by o-rings (4) and special feed through plugs (3). The protruding wire harness was protected using a small boot (2). If a sensor was not needed at a particular location, the o-rings (4) and plugs (5) were used to seal off that hole. An example showing a symmetric 4-sensor arrangement is shown in Figure 32, where the first microphone is placed at $\mathrm{x}_{1}=0.75 \%$, the second at $\mathrm{x}_{2}=3.75 "$, the third at $\mathrm{x}_{3}=8.25$ ", and the fourth at $\mathrm{x}_{4}=11.25$ '. Using the CAD to cut down the midsection of the tube design, Figure 33 shows the arrangement of the microphone sensor in the first recessed hole and the plug components in the second recessed hole. Since the bases of the recessed holes were milled so close in proximity to the upper channel wall, neither the sensors nor the plugs offered any dead volume off the channel (i.e., the fluid could not "see" either unit). Again, careful planning and machining ensured that both the sensor and plug assemblies completely sealed using a pair of rubber o-rings per recession.

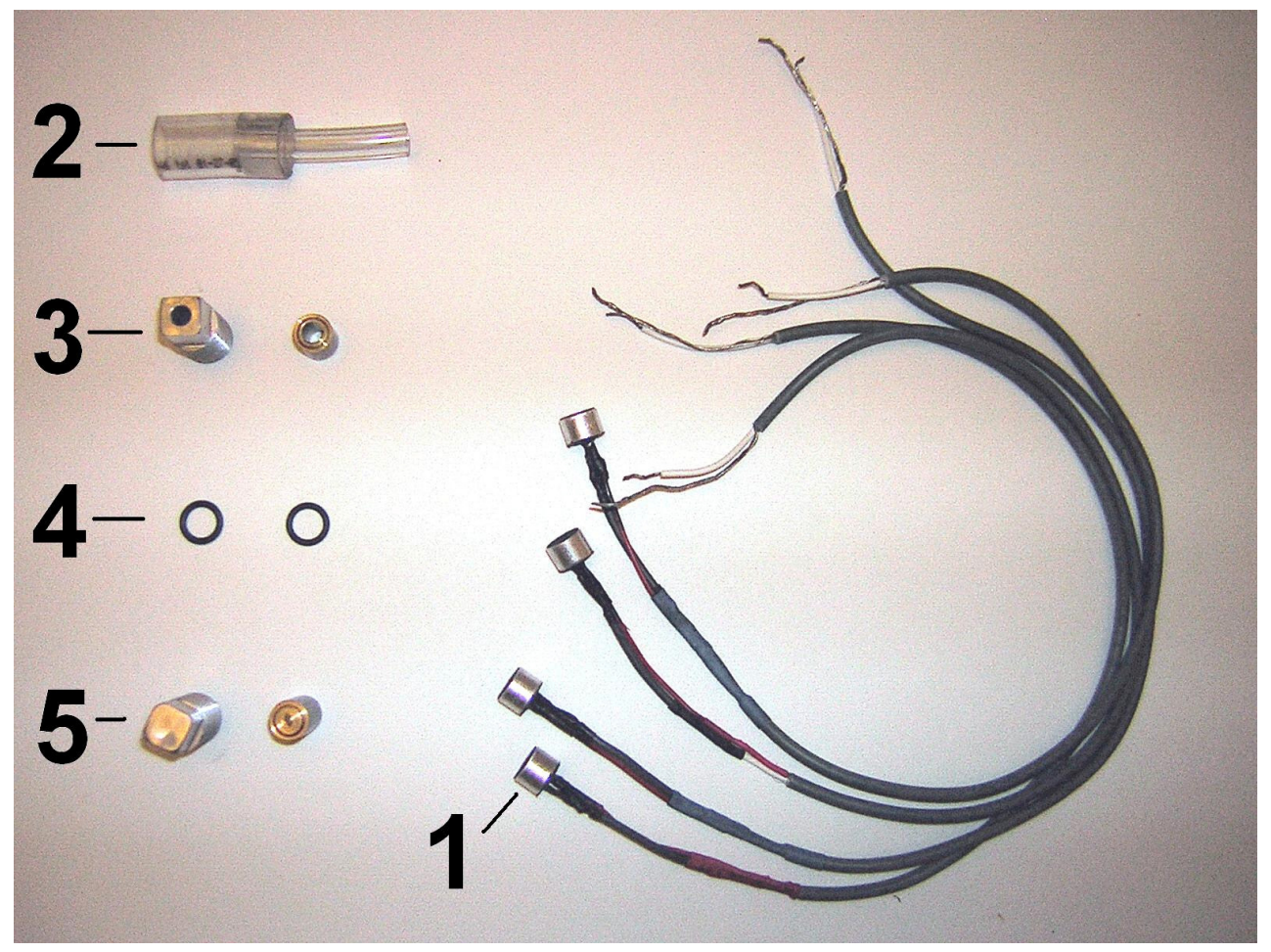

Figure 31: The microphone sensors (1) required some associated hardware (2-5) to outfit the shock tube assembly. 


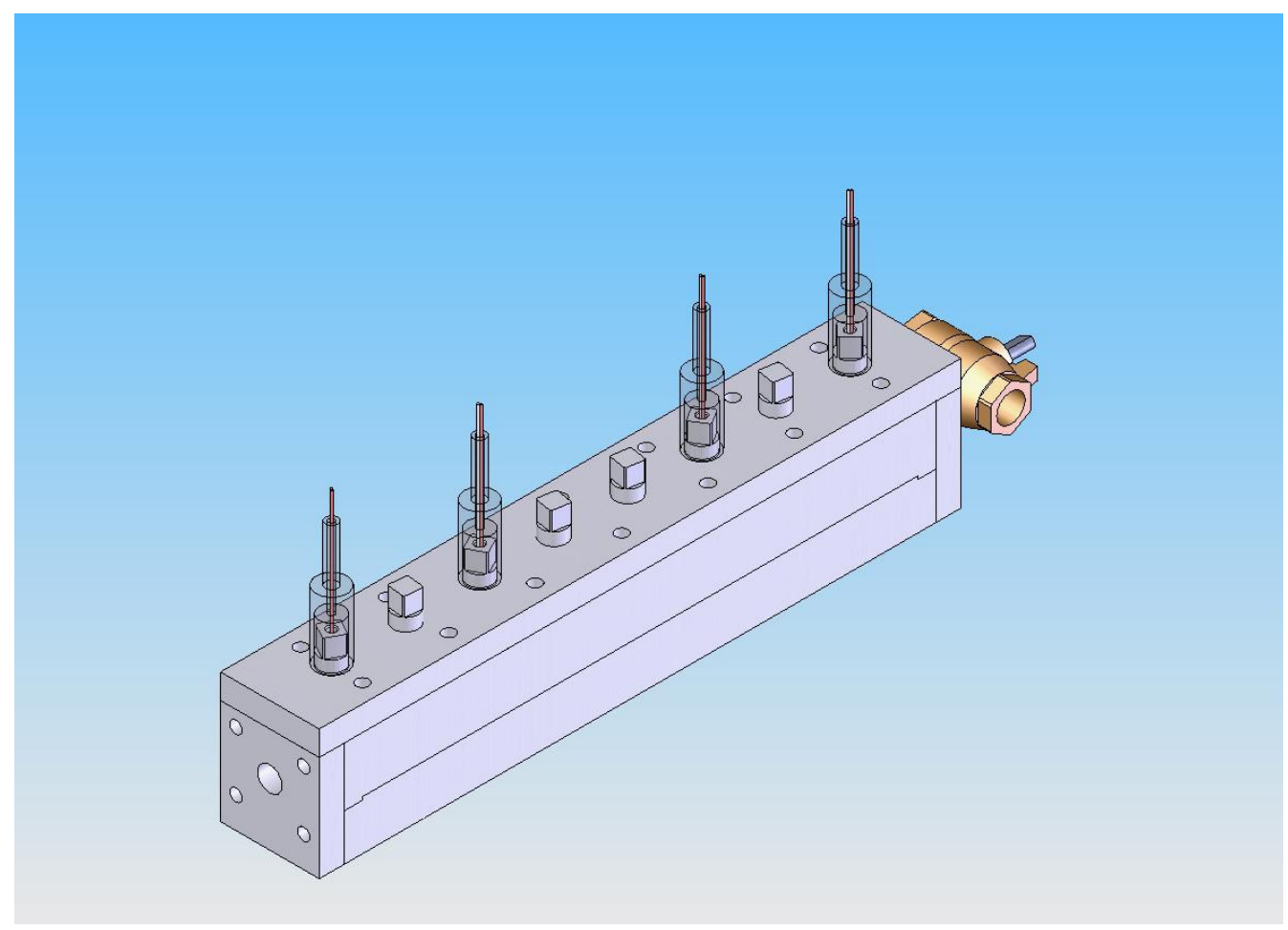

Figure 32: An isometric view of the shock tube CAD model shown with 4 mounted microphone sensors on the top and the CCV assembly mounted at the inlet.

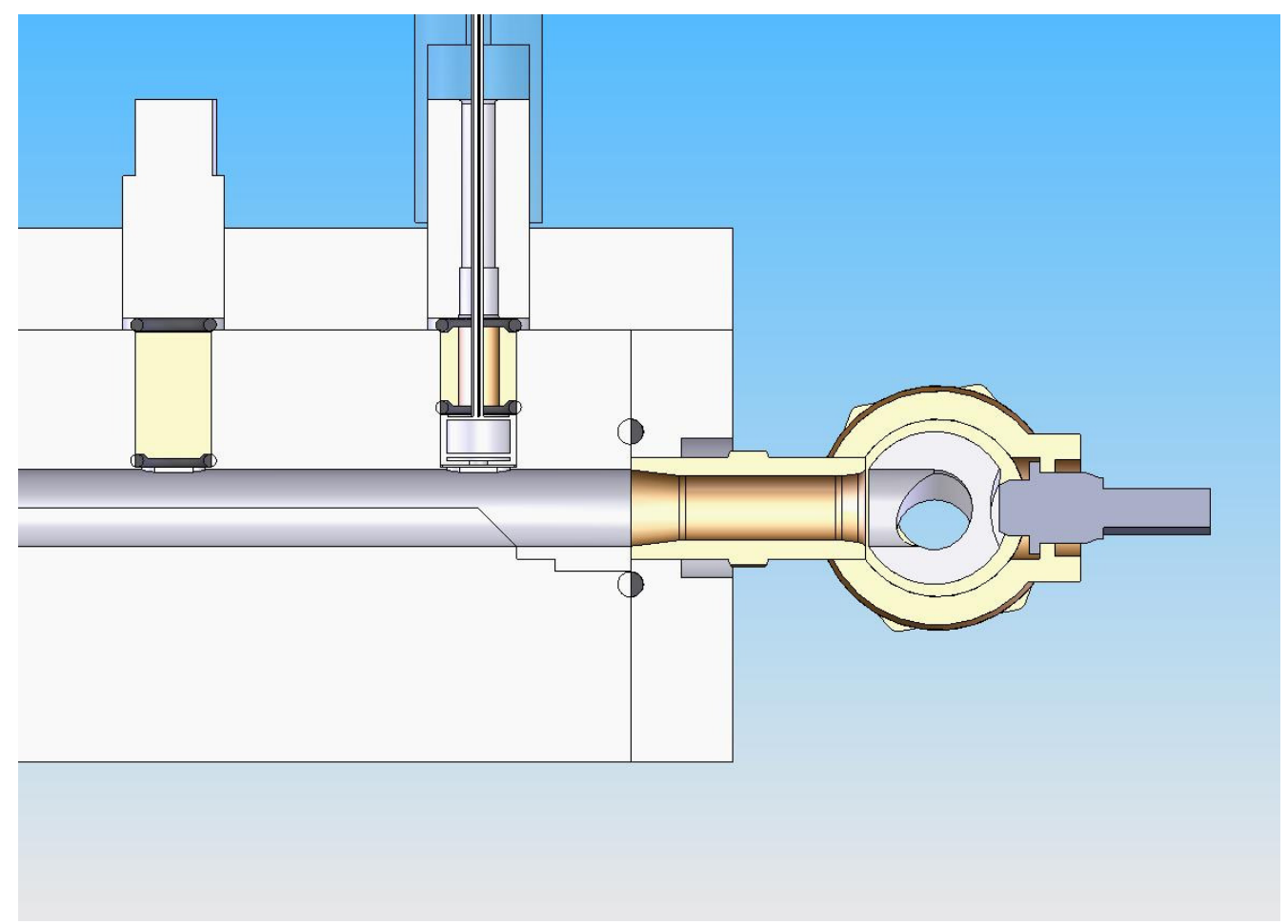

Figure 33: A closer cutaway view shows the CCV assembly, choke nozzle, and shock tube assembly with the first microphone station occupied and the second microphone station unused. 
The final stage of the constant volume experiments involved capturing the injected mass in a positive displacement water collection chamber. Figure 34 shows some aspects of the collection system, which was made up of a sealed rectangular base tank, a lid with the collection chamber cylinder sealed to its underside, a level ruler, and the necessary hose attachment plumbing. As a single cycle of air was injected into the chamber through the inlet, water inside the chamber was displaced downward and the final level was directly read (Figure 35) and recorded to indicate the final pressure in the chamber. The total mass injected during the CCV operation could then be calculated based upon the chamber and reservoir geometry and initial and final heights of the chamber water level. Figure 36 shows the initial and final conditions of a theoretical amount of injected air into the collection system.

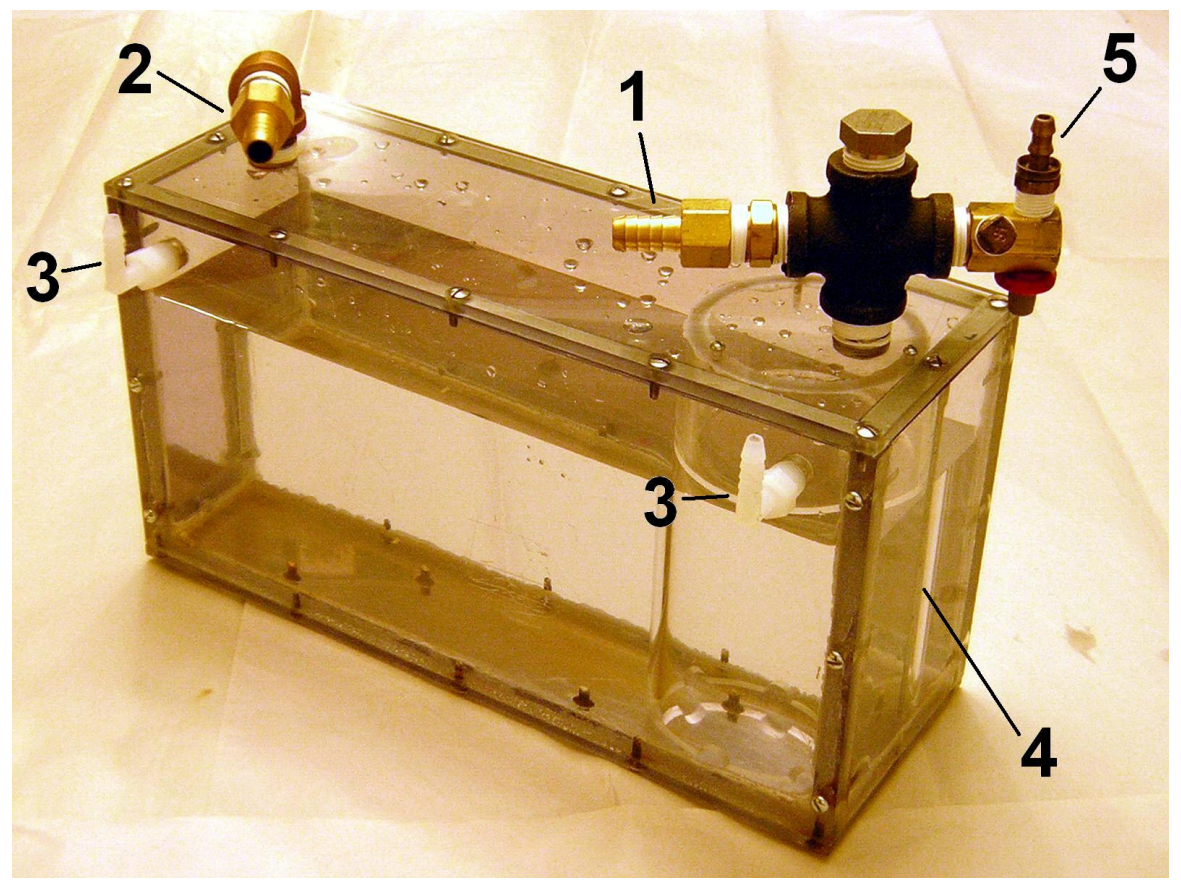

Figure 34: The injected mass collection chamber was made with a single inlet (1), a major vent (2), two minor vents (3), a precision steel rule (4) and an optional throttled chamber pressure tap (5). 


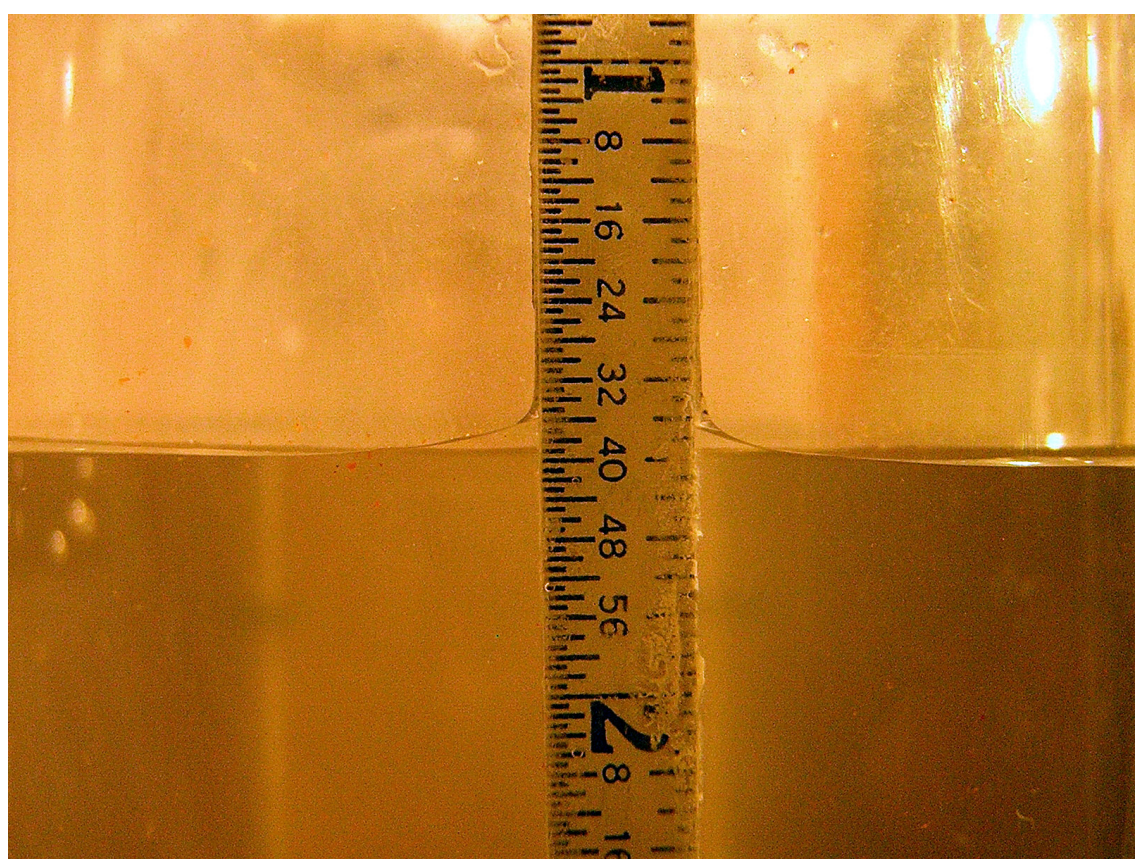

Figure 35: A close up view of the mounted steel rule shows an initial chamber level of $139 / 64$ ”.

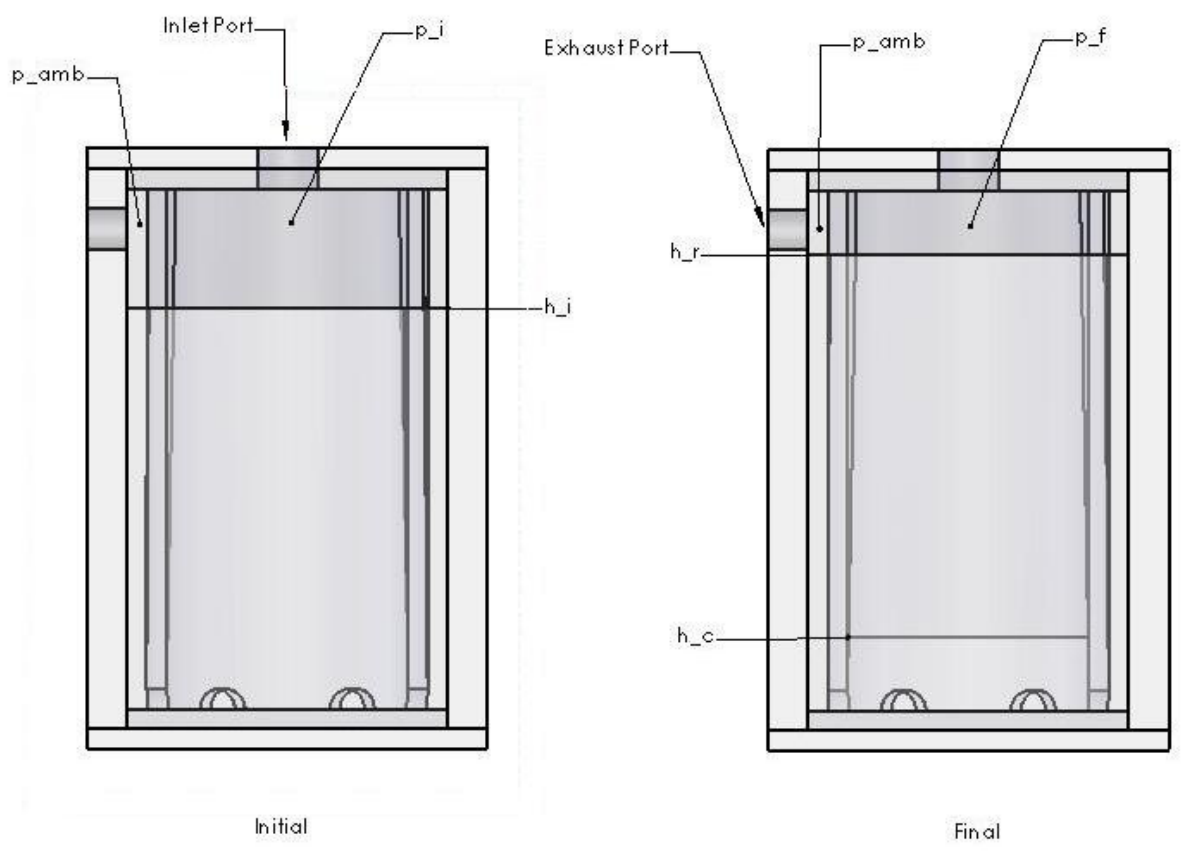

Figure 36: The total injected mass of a single CCV turn was calculated based upon the geometry of the collection chamber and reservoir, and upon the initial and final heights of the chamber water level. 


\section{Test Procedure}

The basic test procedure followed during all the constant pressure tests was as

follows:

1) Turn on the CCV motor to the desired test speed and add or subtract weights to the friction brake according to the test set requirements.

2) Turn on the DAQ system.

3) Ensure that the air regulator master valve is open on the second stage tank.

4) Ensure that the water level in the chamber is set at ambient equilibrium height, $h_{i}$, and that the chamber bleed valve is closed.

5) Start the DAQ software.

6) Record the final chamber height after the injection is complete.

7) Turn off the DAQ system.

8) Turn off the CCV motor.

9) Open the chamber bleed valve.

10) Visually check the CCV stem position and, if necessary, manually realign it by grasping the input shaft adapter.

11) Name the collected data according to the test subset filename code.

Test sets were always begun by a top down speed adjust and a top down pressure adjust. In other words, for a given test matrix the desired speed was approached from the highest speed down. Likewise, a desired pressure ratio between the second stage tank and ambient air pressure was always approached from the highest ratio down. Also, before any major change in sets, the ambient conditions were recorded anew. Sensors 
used during the collection of constant pressure experiments are discussed in more detail in the Data Acquisition section of this chapter. 


\section{Constant Volume Experiments}

\section{General Arrangement and Apparatus}

Constant volume experiments were performed in which gas held within a fixed volume was discharged into an adjoining fixed volume by way of the CCV. Schematically, the setup for these experiments is shown in Figure 37.

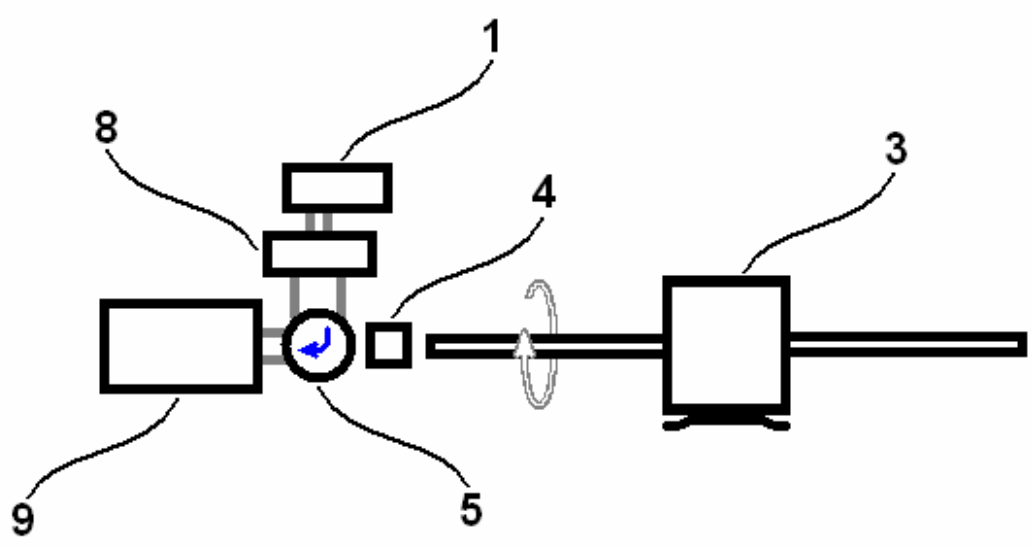

Figure 37: The constant volume experimental setup.

Referring to Figure 37, the motor (3), clutch (4), CCV (5), and regulator (1) remained identical to the constant pressure tests. A relatively small volume (8) was made by adjusting plumbing on the upstream side of the CCV. Downstream, a larger volume was provided again by adjusting the plumbing or adding modified tanks such as the one shown in Figure 38. These larger and smaller volumes were designed to represent the CIBAI cylinder volumes at or near TDC for a common base cylinder displacement. Since the CIBAI method requires the air only cylinder to operate at a much higher compression ratio, the small volume was chosen as the upstream or supply side of the test. Conversely, because the air-fuel side of the CIBAI arrangement must remain at a 
low enough compression ratio to avoid normal knock conditions, the larger volume was selected as the downstream or receiving side of the test.

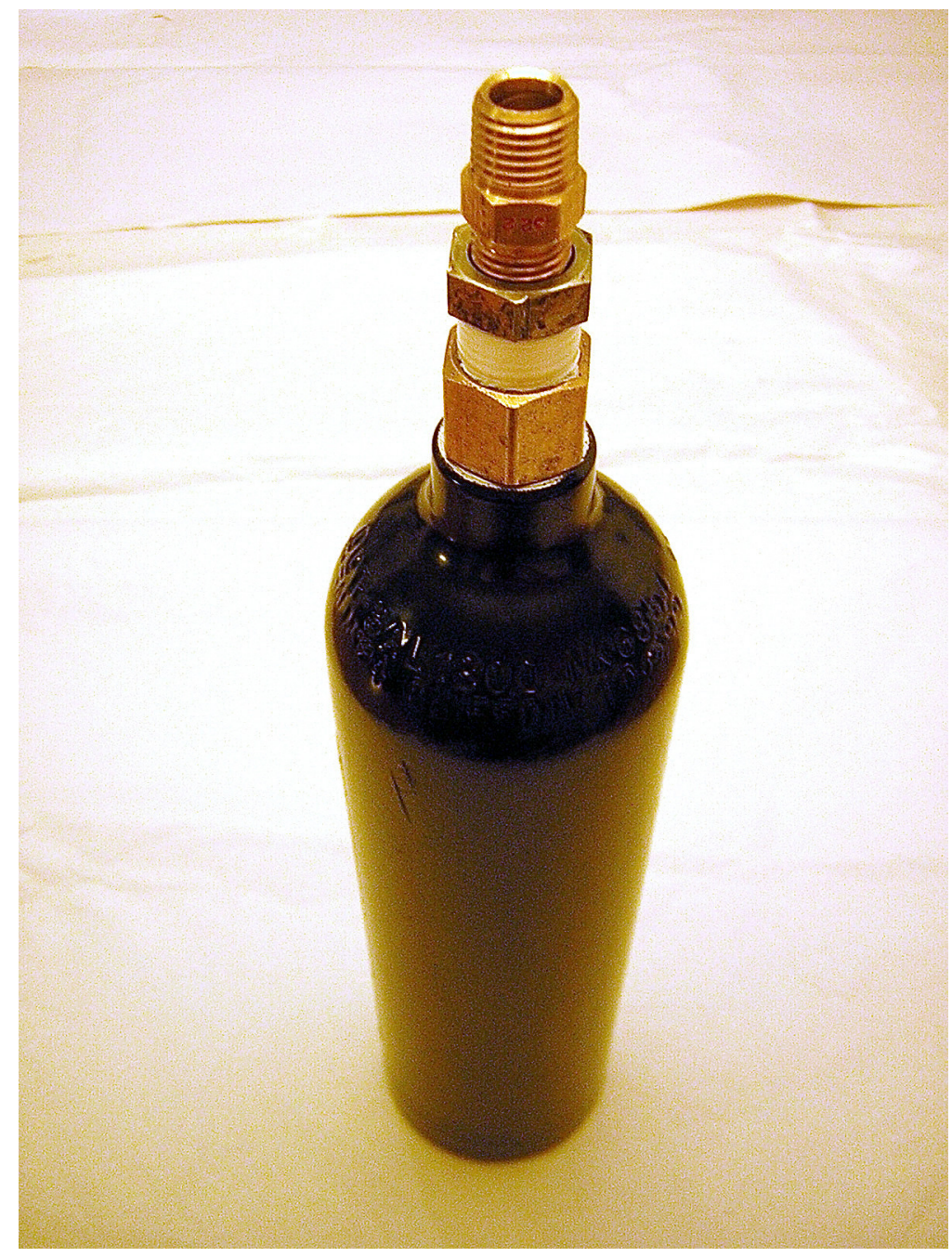

Figure 38: A modified $\mathrm{CO}_{2}$ bottle was used as the downstream constant volume (543cc) tank in many of the constant volume tests.

\section{Test Procedure}

The basic test procedure followed during all the constant volume tests was as follows: 
1) Turn on the CCV motor to the desired test speed and add or subtract weights to the friction brake according to the test set requirements.

2) Turn on the DAQ system.

3) Add air to the low volume side by temporarily opening the air regulator master valve.

4) Once the small volume is fully charged with the intended pressure, close the air regulator master valve.

5) Start the DAQ software.

6) Turn off the DAQ system.

7) Turn off the CCV motor.

8) Visually check the CCV stem position and, if necessary, manually realign it by grasping the input shaft adapter.

9) Name the collected data according to the test subset filename code.

Similarly to the constant pressure tests, these test sets were always begun by a top down speed adjust and a top down pressure adjust. Volumes were changed as one of the parameters of the test matrices by a variety of methods including addition or removal of plumbing, and addition or removal of solid brass plugs of known volume. Like the constant pressure tests, the ambient conditions were continually monitored and recorded between changes in major test sets. Sensors used during the collection of constant volume experiments are discussed in more detail in the Data Acquisition section of this chapter. 


\section{Data Acquisition}

\section{Hardware}

The main hardware used to collect data during experiments consisted of an array of electronic sensors, a basic sensor power supply and wire routing unit called the DAQ center, a Personal Computer Memory Card International Association (PCMCIA) DAQ interface card, and a portable laptop computer. Great care was taken in application of all the equipment to reduce electrical noise as much as possible, and included such steps as housing the battery-powered DAQ center within a shielded box and connecting all sensors using high quality shielded cable. Also, no live AC power lines were allowed near sensor connections or the DAQ center. Table 1 provides a brief overview of the sensors used during the experiments.

Table 1: Electronic sensors were used in a variety of locations to collect the relevant raw data during the experiments.

\begin{tabular}{|l|l|l|}
\hline Sensor & Measurement & Location \\
\hline PX-105 transducer & $\mathrm{p}_{\text {tank }}$ or $\mathrm{p}_{\mathrm{a}}$ & $2^{\text {nd }}$ stage tank or small volume \\
\hline Thermocouple, type $\mathrm{K}$ & $\mathrm{T}_{\text {tank }}$ & $2^{\text {nd }}$ stage tank \\
\hline Magnetic pick up & $\mathrm{RPM}_{\text {motor }}$ & Base of CCV motor assembly \\
\hline Slotted IR & $\mathrm{RPM}_{\text {motor }}$ & Base of CCV motor assembly \\
\hline IR range finder & $\mathrm{RPM}_{\mathrm{CCV}}$ & Bridge beside CCV shaft adapter \\
\hline Capacitive mic \#1-8 & $\Delta \mathrm{p}_{\mathrm{ST}}$ & Shock tube assembly \\
\hline WS-110 & $\mathrm{p}_{\mathrm{amb}}$ and $\mathrm{T}_{\mathrm{amb}}$ & Center of lab room \\
\hline
\end{tabular}

All the internal tank pressures were recorded using the Omega PX-105 high gain, high frequency strain gage pressure transducer. The $10 \mathrm{kHz}$ response frequency was high 
enough to capture an adequate number of data points during a typical injection process and accurately describe its transient behavior. For example, an injection process occurring at a CCV shaft speed of 1068 RPM would exhibit 18.7 milliseconds of open time. Sampling at a rate of $20 \mathrm{kHz}$ to avoid any aliasing problems would be able to resolve the complete range of pressure data and provide 187 reliable data points during the injection.

A common type $\mathrm{K}$ thermocouple and corresponding signal conditioner were used to indicate the secondary stage tank temperature during all experiments. The thermocouple was mounted against the sidewall of the tank using reflective steel tape which was then covered by a layer of insulating foam, followed by a layer of rigid plastic which was attached using a second layer of reflective steel tape. Initially, the digital signal was recorded during several early experiments, but the fluctuation in tank temperature was so minor $\left(<1^{\circ} \mathrm{F}\right)$ that it did not warrant the use of an entire recorded input channel. The remaining bulk of experiments used the thermocouple data as a single reference data point which was manually recorded on corresponding data set reference sheets.

Figure 39 shows the magnetic switch and IR beam splitter arrangement used to indicate the speed and relative position of the $\mathrm{CCV}$ drive motor. The slotted disk was $\mathrm{CNC}$ milled and balanced to provide $15^{\circ}$ resolution of the $\mathrm{CCV}$ motor position which provided 24 pulses per revolution of the drive shaft. The magnetic switch recorded only one pulse per revolution, but was installed initially to ensure the electronic accuracy of the slotted IR sensor. After many tests, there was no doubt that the IR sensor provided reliable pulses, so the redundant magnetic circuit was later disconnected. 


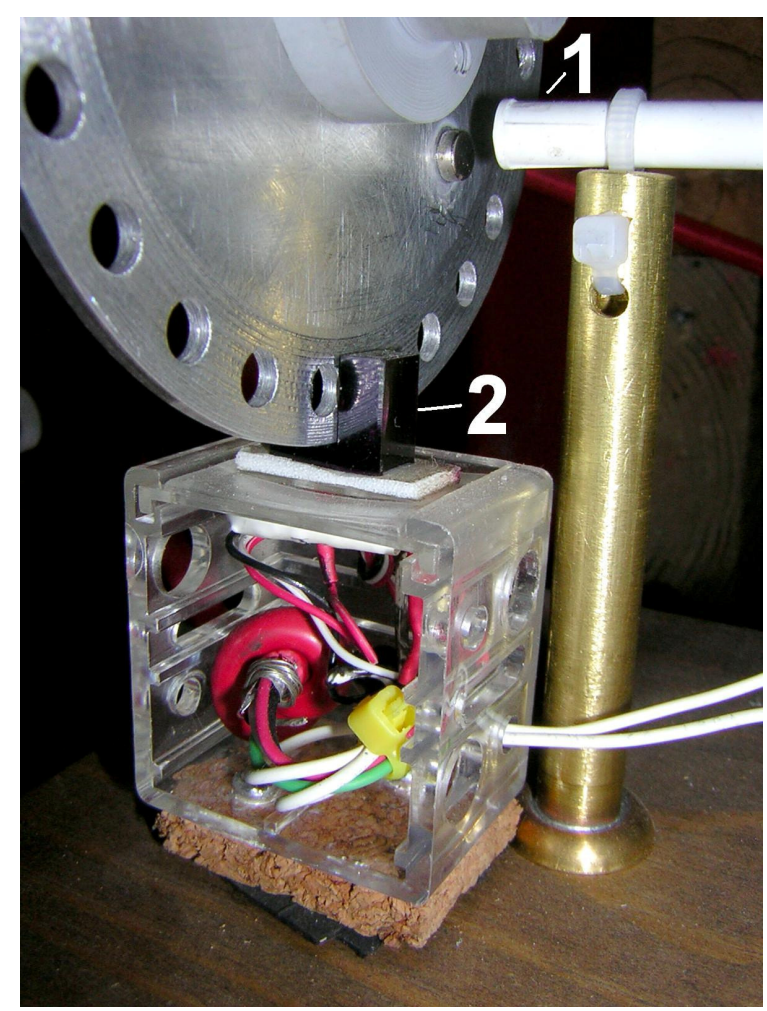

Figure 39: A magnetic pickup switch (1) and a slotted IR beam splitter (2) mounted below the CCV motor shaft were used to indicate the motor speed and general position.

The omnidirectional capacitive discharge microphone model (Figure 40) used in the shock tube assembly offered a robust method of investigating highly transient fluid pressure behavior inside the tube. A response frequency of $22 \mathrm{kHz}$ provided more than enough resolution to capture the high speed passage of a starting shock wave as it passed through the tube. It was found during initial experiments that exposure to high pressure had the potential of saturating the return signal from the microphone, but caused no permanent alterations to the sensor's response behavior. 


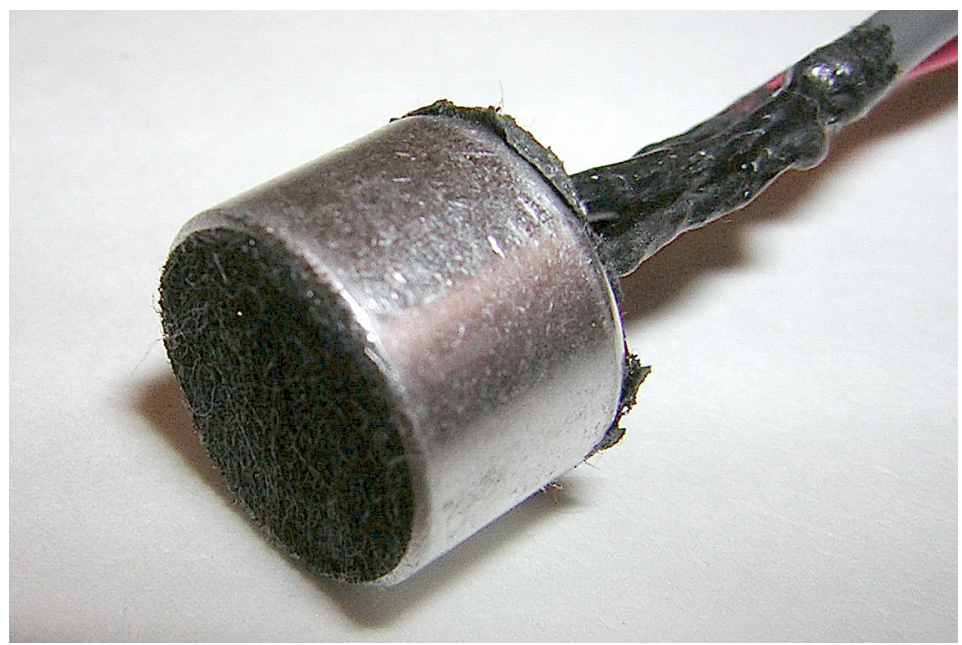

Figure 40: The fast response microphone used in the shock tube experiments.

Ambient conditions for each test subset were manually recorded in a fashion similar to the tank temperature on a corresponding data set reference sheet. Using the digital weather station WS-110, the ambient temperature was recorded in ${ }^{\circ} \mathrm{F}$ and the ambient pressure was recorded in $\mathrm{Pa}$. Ambient conditions were recorded for every subset, even if no change in room conditions had occurred between experiments.

Both the supply voltages and the response signals from the sensors were maintained by the DAQ center which served as the central hub for all data acquisition. Referring to Figure 41, the main power switch (1) was used to control power to the rest of the sensor network. The 27 VDC power supply (3) energized the entire system and was restricted in its total current output by a 1 amp fuse. Input and output signals were transmitted between the PCMCIA card and the sensors by way of the 37 pin Omega DAQPA cable (2) and shielded cables (5). Because the power required to energize the clutch circuit was much higher than the power delivery capabilities of the PCMCIA card, a multi step circuit was required to enable computer control of the clutch. The low power signal was first received by a 555 timer integrated circuit (IC) (4) which was then used to 
trigger a higher power relay circuit which was in turn used to trigger an even higher power excitation signal used to activate the clutch. Finally, the whole DAQ center was sealed using the air tight aluminum lid (6) which was also used to hold the quick reference DAQ circuit schematic.

The PCMCIA DAQ card (Omega DAQP-208H, Figure 42) used for the experiments offered high speed $(100 \mathrm{kHz})$ collection of good resolution (16-bit) data sampling. The card provided a maximum of 8 single-ended (common ground) input channels or 4 double-ended (floating ground) input channels with a maximum voltage range of \pm 10 VDC. Additionally, the card allowed up to two 12-bit digital output channels with a maximum voltage range of \pm 5 VDC. An important consideration when adding sensors was that the card limited the total collection of data points to 100,000 per second. Thus, for an arrangement using 4 single ended channels, the actual maximum scan rate became $(100 / 4) \mathrm{kHz}$, or $25 \mathrm{kHz}$. Optimizing the scanning ability of the DAQ card meant arranging the software so that only the necessary channels would be used. By the end of the experiments at least six different channel scanning arrays were programmed into the DAQ software. Table 2 shows these arrangements with the corresponding sensors being used in each setup. An additional important aspect of the scanning method of the card was that every channel scan recorded the data points for the input channels simultaneously. Looking again at the four channel example above, this meant that at the generic scan number $\mathrm{N}$, the $\mathrm{N}^{\text {th }}$ data entries for channels 1 through 4 occurred at the same instant in time. 


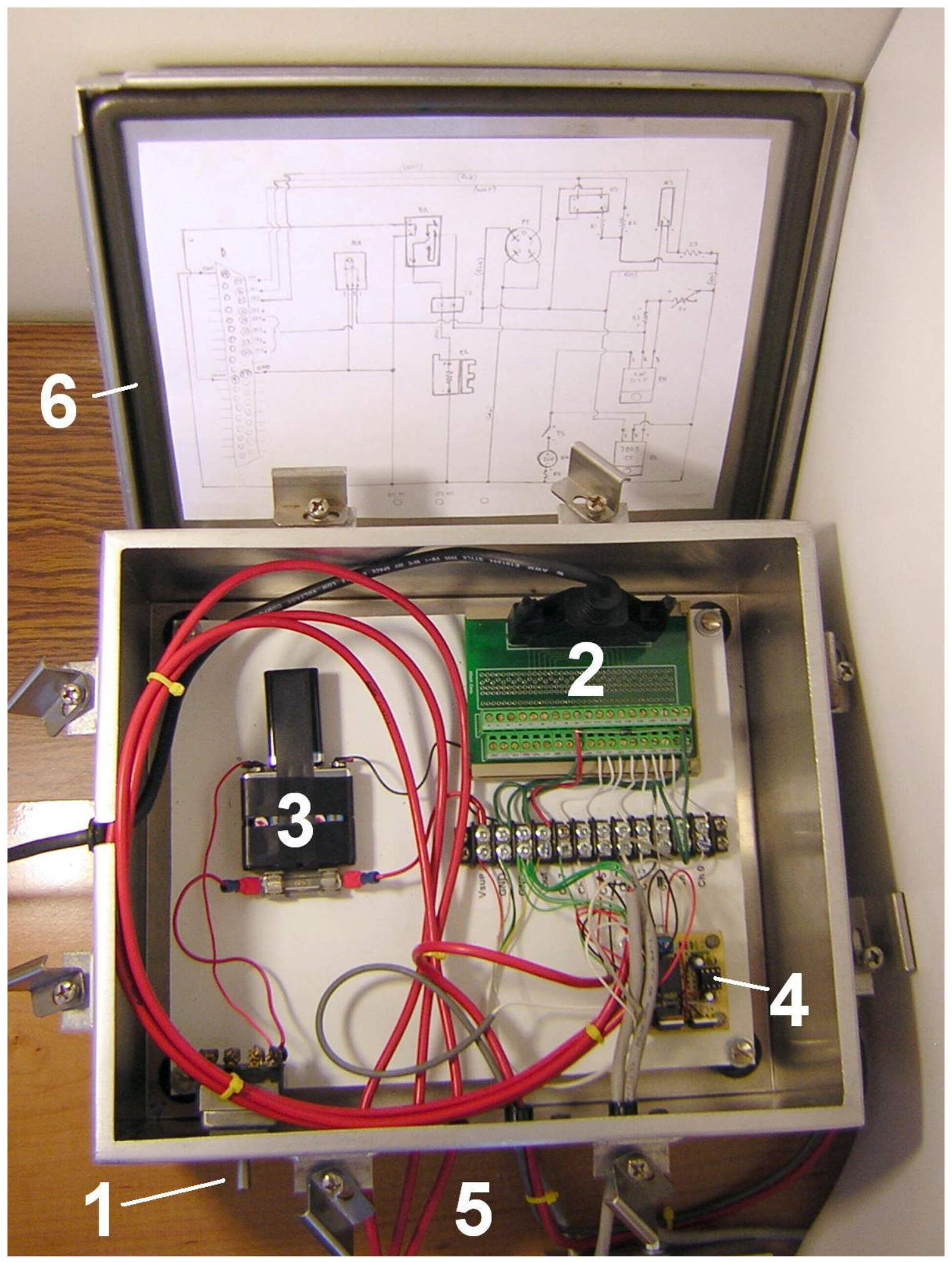

Figure 41: The DAQ center was entirely contained within a 12" x 10" x 5" aluminum box. 


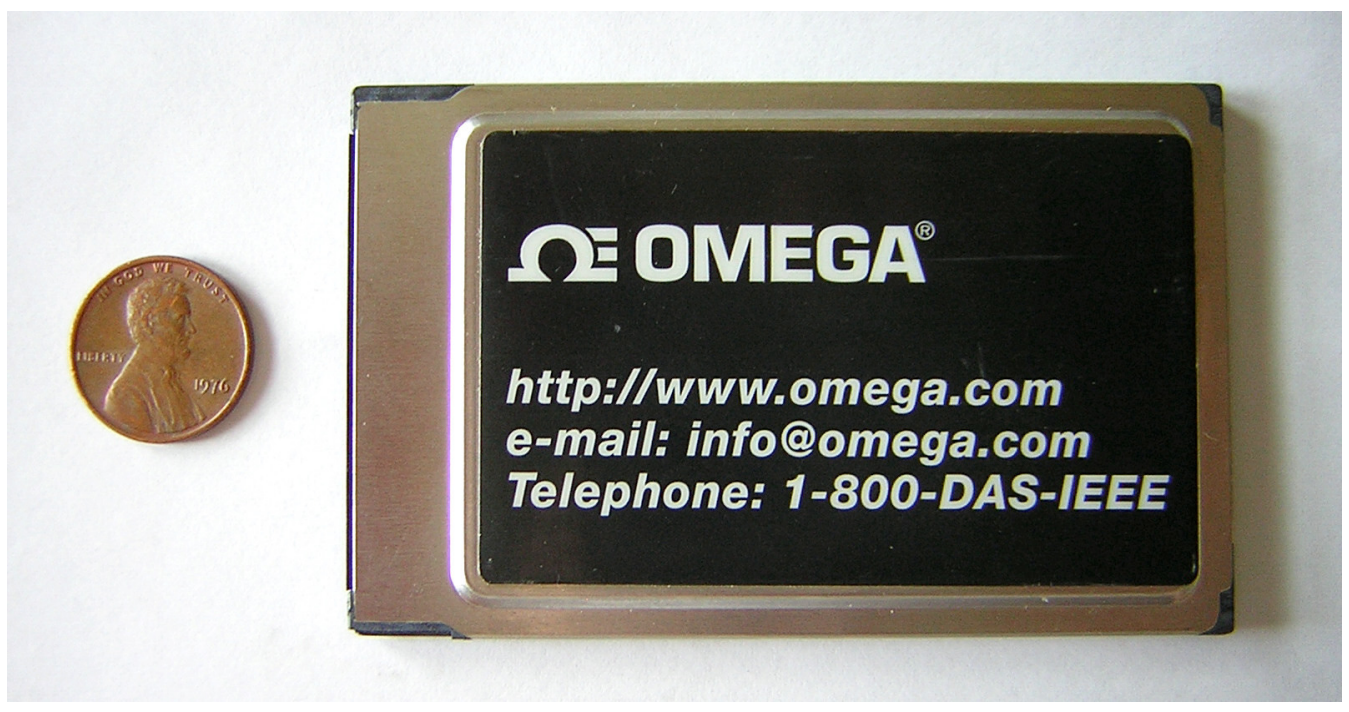

Figure 42: The Omega DAQP-208H PCMCIA card was used to collect experimental data.

Table 2: Six different input channel arrangements were used during different stages of experiments to maximize the allowable scanning frequency.

\begin{tabular}{|c|c|c|c|c|c|c|}
\hline Ch. Array Name & STD & SP1 & SP2 & SP3 & SP4 & SP5 \\
\hline $\begin{array}{l}\text { Sampling rate, } f_{s} \\
(\mathrm{kHz})\end{array}$ & 12.5 & 25 & 50 & 50 & 100 & 33.333 \\
\hline Channel 0 & $\mathrm{p}_{\text {tank }}$ & $\mathrm{p}_{\text {tank }}$ & $\mathrm{p}_{\text {tank }}$ & $\mathrm{p}_{\text {tank }}$ & $\mathrm{p}_{\text {tank }}$ & $(\mathrm{N} / \mathrm{A})$ \\
\hline Channel 1 & $\mathrm{~T}_{\text {tank }}$ & $(\mathrm{N} / \mathrm{A})$ & $(\mathrm{N} / \mathrm{A})$ & $(\mathrm{N} / \mathrm{A})$ & $(\mathrm{N} / \mathrm{A})$ & $(\mathrm{N} / \mathrm{A})$ \\
\hline Channel 2 & POS $_{\mathrm{MOT}}$ & $(\mathrm{N} / \mathrm{A})$ & $(\mathrm{N} / \mathrm{A})$ & POS $_{\mathrm{MOT}}$ & $(\mathrm{N} / \mathrm{A})$ & $\mathrm{POS}_{\mathrm{MOT}}$ \\
\hline Channel 3 & $\mathrm{POS}_{\mathrm{CCV}}$ & $\mathrm{POS}_{\mathrm{CCV}}$ & $\mathrm{POS}_{\mathrm{CCV}}$ & $(\mathrm{N} / \mathrm{A})$ & $(\mathrm{N} / \mathrm{A})$ & $(\mathrm{N} / \mathrm{A})$ \\
\hline Channel 4 & $\mathrm{MIC}_{1}$ & $\mathrm{MIC}_{1}$ & $(\mathrm{~N} / \mathrm{A})$ & $(\mathrm{N} / \mathrm{A})$ & $(\mathrm{N} / \mathrm{A})$ & $\mathrm{MIC}_{1}$ \\
\hline Channel 5 & $\mathrm{MIC}_{2}$ & $(\mathrm{~N} / \mathrm{A})$ & $(\mathrm{N} / \mathrm{A})$ & $(\mathrm{N} / \mathrm{A})$ & $(\mathrm{N} / \mathrm{A})$ & $(\mathrm{N} / \mathrm{A})$ \\
\hline Channel 6 & $\mathrm{MIC}_{3}$ & $(\mathrm{~N} / \mathrm{A})$ & $(\mathrm{N} / \mathrm{A})$ & $(\mathrm{N} / \mathrm{A})$ & $(\mathrm{N} / \mathrm{A})$ & $(\mathrm{N} / \mathrm{A})$ \\
\hline Channel 7 & $\mathrm{MIC}_{4}$ & $\mathrm{MIC}_{4}$ & $(\mathrm{~N} / \mathrm{A})$ & $(\mathrm{N} / \mathrm{A})$ & $(\mathrm{N} / \mathrm{A})$ & $\mathrm{MIC}_{4}$ \\
\hline
\end{tabular}


It may be deduced from Table 2 that only the standard (STD), specialized \#1 (SP1), and specialized \#5 (SP5) channel arrays were used to study flow behavior in the shock tube assembly since they all include microphone input collection. The SP1 array was the major arrangement used to determine tube flow behavior with respect to the particular behavior of the CCV position, and the behavior was studied again at a slightly higher frequency using the SP5 array with emphasis on higher accuracy of the indicated motor speed. SP2, SP3, and SP4 arrays were used during the constant volume experiments.

The final hardware component used in the DAQ system was the portable laptop computer. The Dell Inspiron 2650 powered by a $1.8 \mathrm{GHz}$ Intel Pentium $4 \mathrm{CPU}$ and a modest $512 \mathrm{MB}$ of RAM was quite capable of handling acquisition and storage of the incoming data stream. It should be noted that while it was possible to run experiments using the main computer's clock, the DAQ clock speeds were instead managed using the DAQP-208H internal clock as recommended by Omega. 


\section{Chapter 6: Results and Discussion}

\section{Shock Tube Results}

Based upon initial calibration of the microphone sensors, it was possible to infer a huge amount of information about the transient fluid behavior within the tube during the entire recorded test time. Figure 43 shows a typical example of a paired microphone response plot over an entire test time of 0.10 seconds, and Table 3 provides a brief summary of the associated behavioral regions. Referring to Figure 43, a "dead" or static zone (A) is exhibited during the initial phase of the fluid behavior. During this time period, the CCV clutch is disengaged, the CCV is in a fixed closed position, and the major stimuli for the sensors are divided between weak frame vibrational energy and electrical noise. The entire time of CCV clutch engagement, i.e., the time when the $\mathrm{CCV}$ is actually turning, is shown as zone (B). This operational time zone exhibits 3 separate phases within the tube. The first phase shows a strong frame vibration zone (D), which is caused entirely by the friction experienced by the rotating ball within the CCV housing. The second phase shows a strong unique pressure response zone (E), which is caused by the sudden passage of the moving fluid that carries with it a region of stepped-up pressure. Shock tube theory already discussed calls the ratio of this sudden pressure rise $\mathrm{P}_{21}$. The third phase shows strong acoustic response zone (F), during which time all the other wave structures associated with the tube flow behave according to rapidly changing local acoustics. It should be noted that zone (F) is really the part of the fluid flow most people are familiar with- it is simply air which is noisily leaking though a hole into room air. Upon closing the $\mathrm{CCV}$ and disengaging the $\mathrm{CCV}$ clutch, sensors exhibit strong low 
frequency pressure response in zone (C). This behavior is clearly dominated by low frequency pressure oscillations known as Helmholtz resonance. The moment the CCV closes, air inside the single ended tube begins to drop in density as air further upstream continues to move out. The vacuum causes the air to reverse direction, and the subsequent oscillation ensues. It is worth noting that this phenomenon is quite useful in acquiring a frequency-based measurement of the local speed of sound which for a (nearly) constant area tube of length L is given by

$$
a=2 \pi L f_{H} .
$$

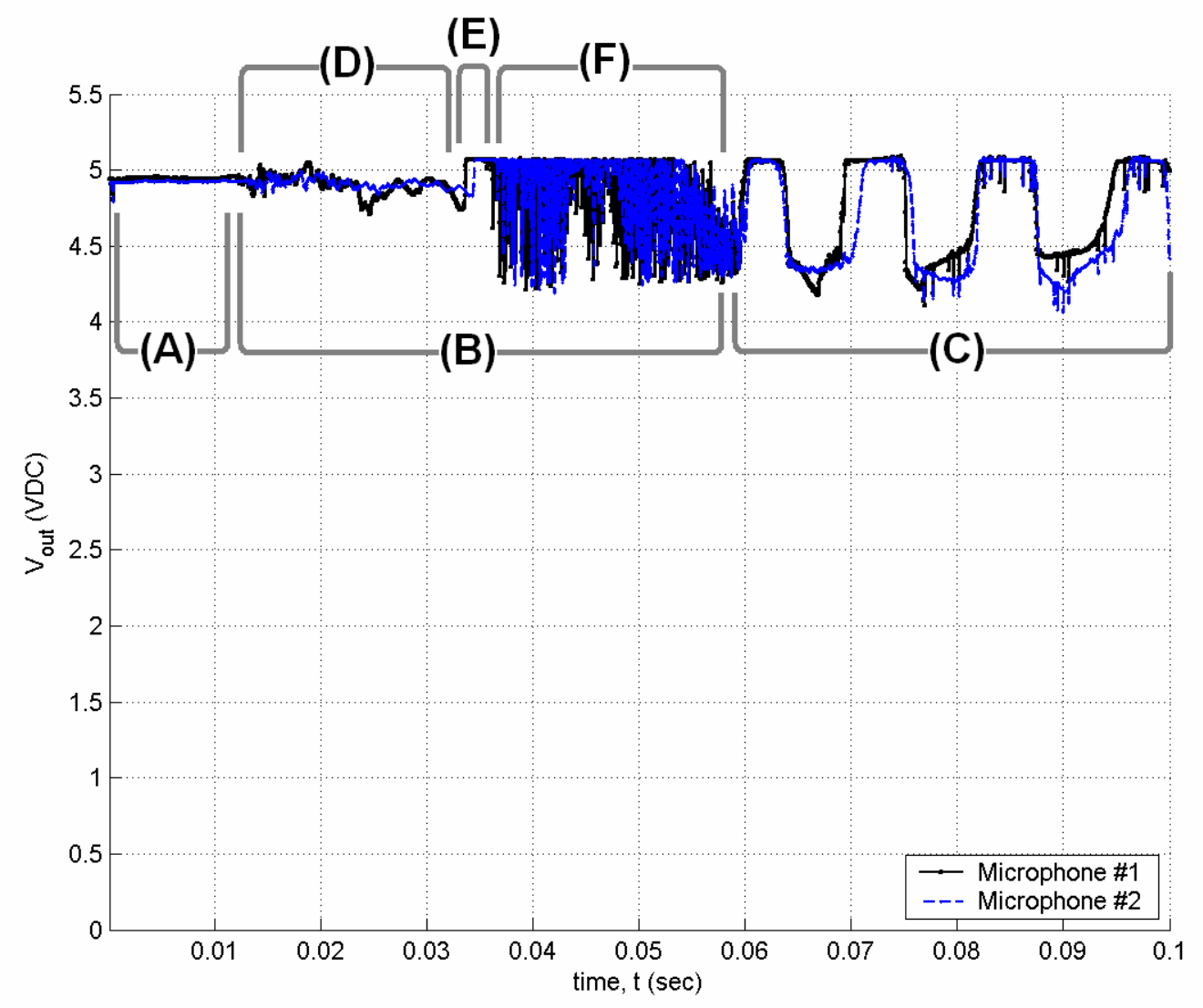

Figure 43: An example of a dual-microphone sensor response during an injection process reveals at least 5 distinct transient behavioral patterns. 
Table 3: The distinct transient behavioral patterns are easily categorized according to the instantaneous CCV behavior and the corresponding dominant stimulus.

\begin{tabular}{|c|c|c|c|c|c|}
\hline \multirow{2}{*}{\multicolumn{2}{|c|}{ Zone }} & \multirow[b]{2}{*}{ CCV behavior } & \multicolumn{2}{|c|}{ Sensor response } & \multirow[b]{2}{*}{ Dominant stimulus } \\
\hline & & & (rel. freq.) & (rel. amp.) & \\
\hline \multicolumn{2}{|c|}{$\mathbf{A}$} & closed, not turning & high & low & $\begin{array}{l}\text { minor frame vibration \& electrical } \\
\text { noise }\end{array}$ \\
\hline \multirow{3}{*}{ B } & $\mathbf{D}$ & closed, turning & moderate & moderate & frame vibration (CCV friction) \\
\hline & $\mathbf{E}$ & open, turning & unique & $\begin{array}{l}\text { high } \\
\text { (saturated) }\end{array}$ & fluid over-pressure (shock passage) \\
\hline & $\mathbf{F}$ & open, turning & high & high & fluid acoustics (rapid fluid passage) \\
\hline \multicolumn{2}{|c|}{$\mathbf{C}$} & closed, not turning & low & high & $\begin{array}{l}\text { fluid pressure (Helmholtz } \\
\text { resonance) }\end{array}$ \\
\hline
\end{tabular}

The region of highest interest to the research was the unique fluid over-pressure behavior of zone (E). Examining the trends in more detail (Figure 44) shows how it is possible to determine the speed at which the potential starting shock moves through the tube. For example, in the case shown, the time difference, $\Delta$ t, between instants of sensor "recognition" of the high pressure zone behind the passing shock was found to be 0.76 milliseconds. Because the distance between the two sensors is known (in this case, $\Delta \mathrm{x}=$ 10.500 " or $0.875 \mathrm{ft}$ ), the velocity of the passing high pressure region was found to be $\Delta \mathrm{x} / \Delta \mathrm{t}=1150$ feet per second. The known ambient conditions of the test, in this case $\mathrm{T}_{\mathrm{amb}}=77.5^{\circ}$, gave a known ambient speed of sound which was found to be 1136 feet per second. Recalling shock tube theory's definition of the starting shock wave Mach number,

$$
M_{S}=\frac{w_{1}}{a_{1}}=W_{11}=\sqrt{\beta_{1}\left(1+\alpha_{1} P_{21}\right)},
$$


the shock Mach number was found to be $\mathrm{M}_{\mathrm{S}}=1.01$.

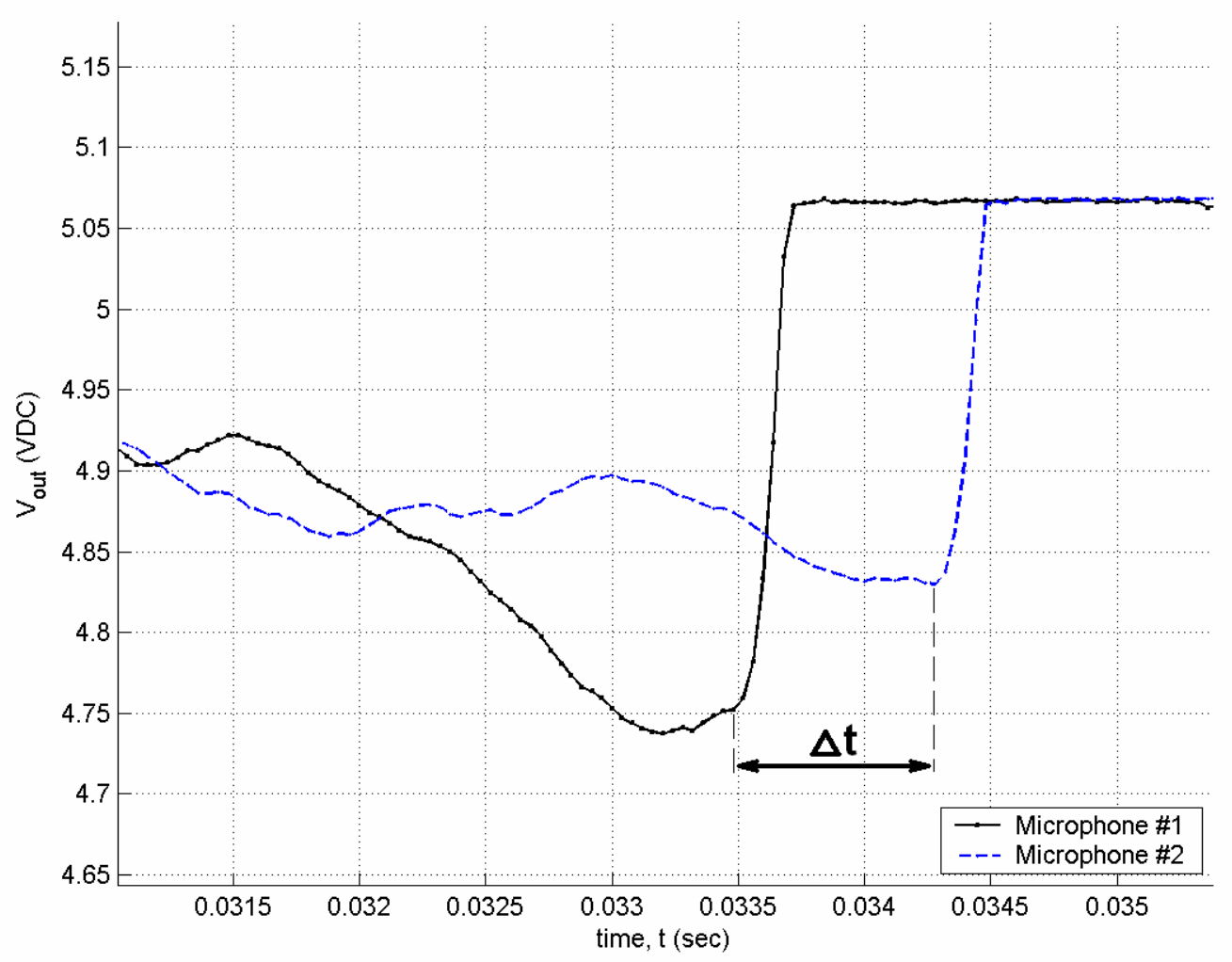

Figure 44: A closer inspection of the microphone signals indicates the time lag $(\Delta t)$ between the first and second microphones' indications of sudden pressure rise.

Theoretically, for the applied pressure ratios that ranged from $1.68<r_{p}<5.76$, the corresponding shock Mach numbers should have ranged from $1.12<\mathrm{M}_{\mathrm{S}}<1.44$. However, as shown in Figure 45 the experimentally measured values of "shock" Mach numbers were found to be nearly sonic in every test $\left(0.96<\mathrm{M}_{\mathrm{S}}<1.07\right)$, indicating that the actual fluid movement revealed by the sensors was not a shock wave but rather a sonic pressure front. This revelation was by no means a surprise, considering the potential for high relative surface roughness within the ball and the turning angle 
experienced by the flow as it moved through the modified CCV ball. While perhaps not obvious, there was a measurable benefit to such a condition, namely that the network of oblique shocks required to lower the flow Mach number also reduced the net shock loss experienced by the flow. In other words, rather than experiencing a single dramatic normal starting shock and the high total pressure loss involved with such an event, the existence of several oblique shocks and their associated low total pressure loss led to a sonic pressure wave. This reduced-loss condition led to higher than anticipated mass flow rates.

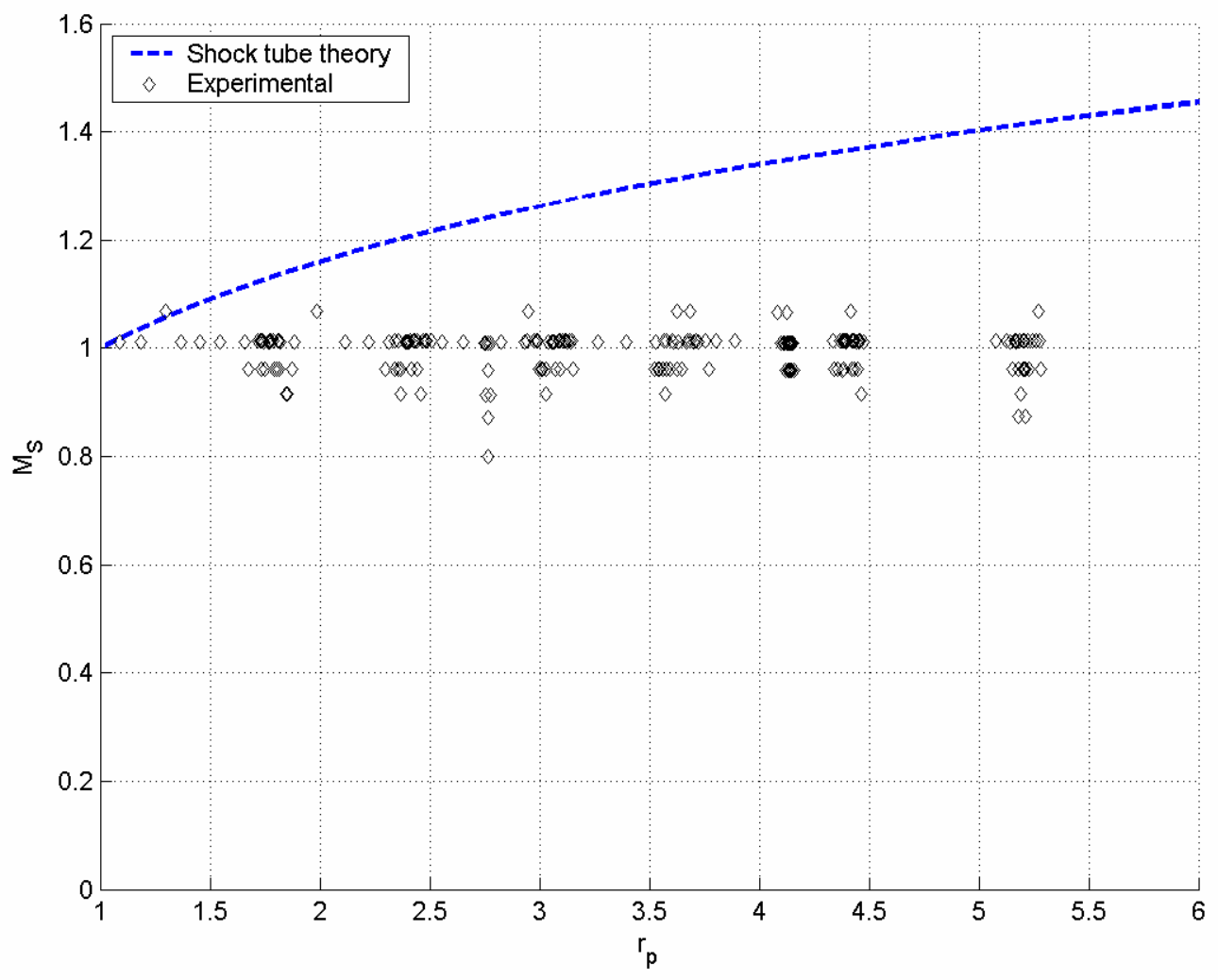

Figure 45: The measured Mach number of the supposed starting shock wave indicated that the initial sudden pressure rise was actually caused by a Mach wave traveling sonically through the tube. 


\section{Constant Pressure Results}

Results for a typical series of constant pressure mass injection tests are provided in the figures that follow. Each set was investigated over a range of pressure ratios and CCV speeds according to a generic matrix shown in Table 4. The particular example set shown is the 150 -sample 4000 series (recorded as sample4201.txt through sample4630.txt).

Table 4: A test matrix was used for each test series " $x$ " in which the mass injection behavior as a function of CCV speed and pressure ratios was systematically investigated.

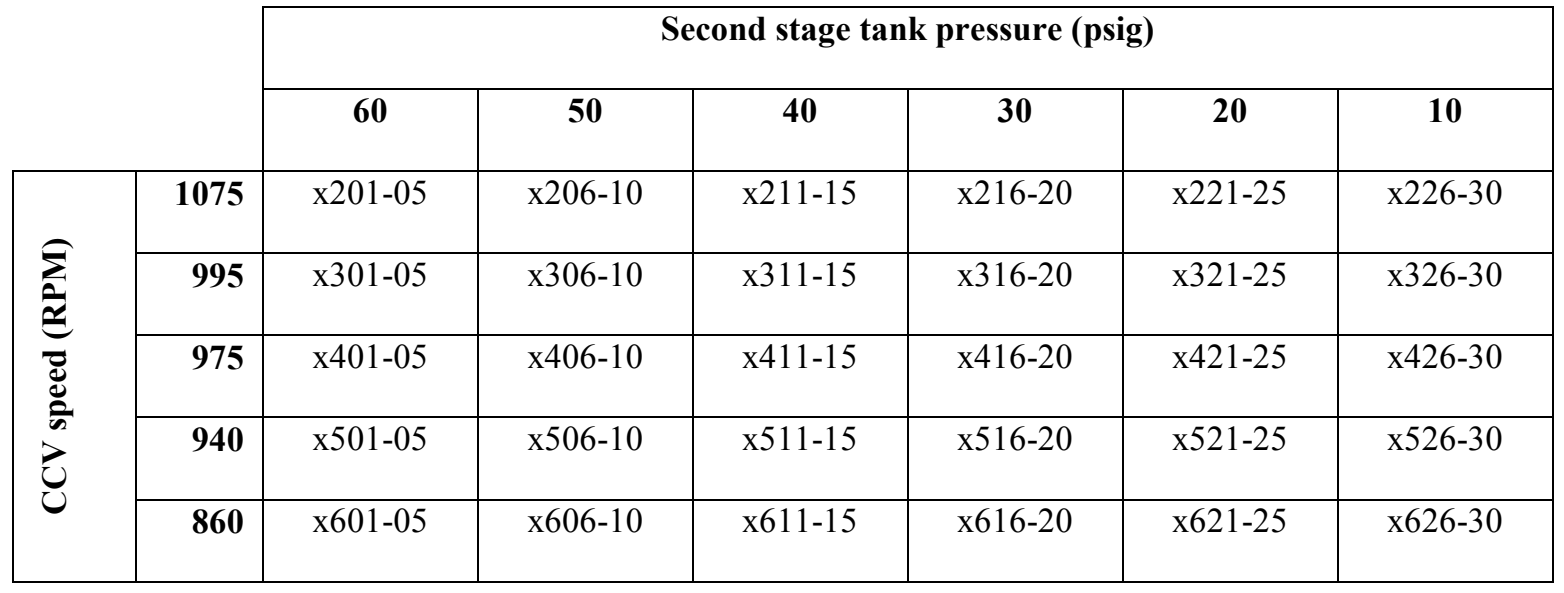

Figure 46 through Figure 51 present the measured values of injected mass as a function of CCV speed plotted in comparison to the theoretically expected trends based upon choked flow theory and shock tube theory. The steady isentropic choked flow theory serves as an upper bound while the irreversible behavior predicted by shock tube theory serves as a lower bound. It is important to note that some minor variation in the "fixed" pressure ratio is exhibited for all the test subsets which examined injection mass as a function of CCV speed only. The main cause of the noted variation was the 
hysteresis effect evident in the second stage supply air regulator which required several injection events to occur before opening to add more charge air to the tank.

Figure 52 through Figure 56 show the measured values of injection mass as a function of constant pressure ratios, again comparing the data to the theoretical bounds already discussed. It is important to note that the calculated likely error for the measured injection mass was $\pm 2.972 \%$. Similar to the minor fluctuation in pressure ratios, the fixed speed tests displayed some minor variation in CCV operating speeds. The main cause of variation in the $\mathrm{CCV}$ speeds was the friction brake heat-fade effect which tended to gradually reduce load resistance to the motor so that the motor speed increased gradually with time.

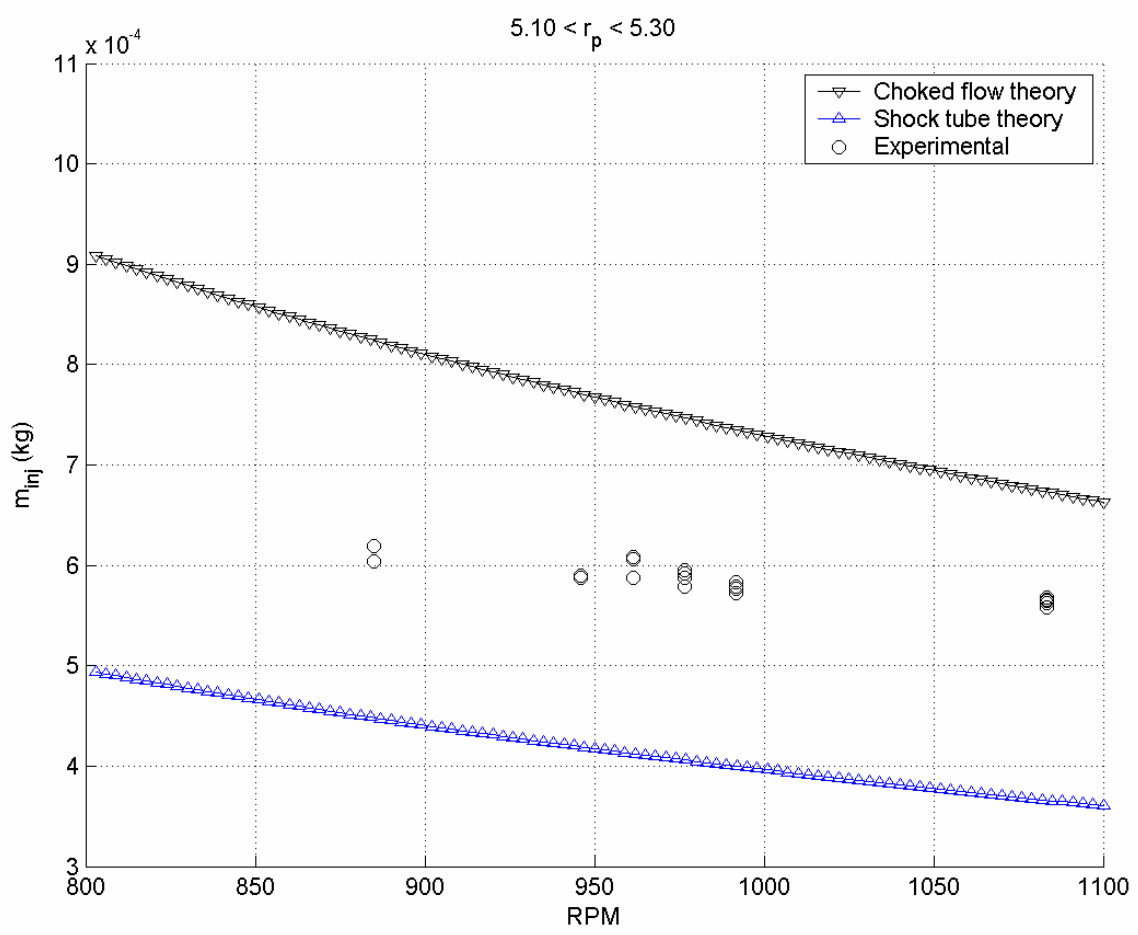

Figure 46: Injected mass from all test samples xx01 through xx05 ( $\left.\mathrm{p}_{\text {tank }} \approx 60 \mathrm{psig}\right)$. 


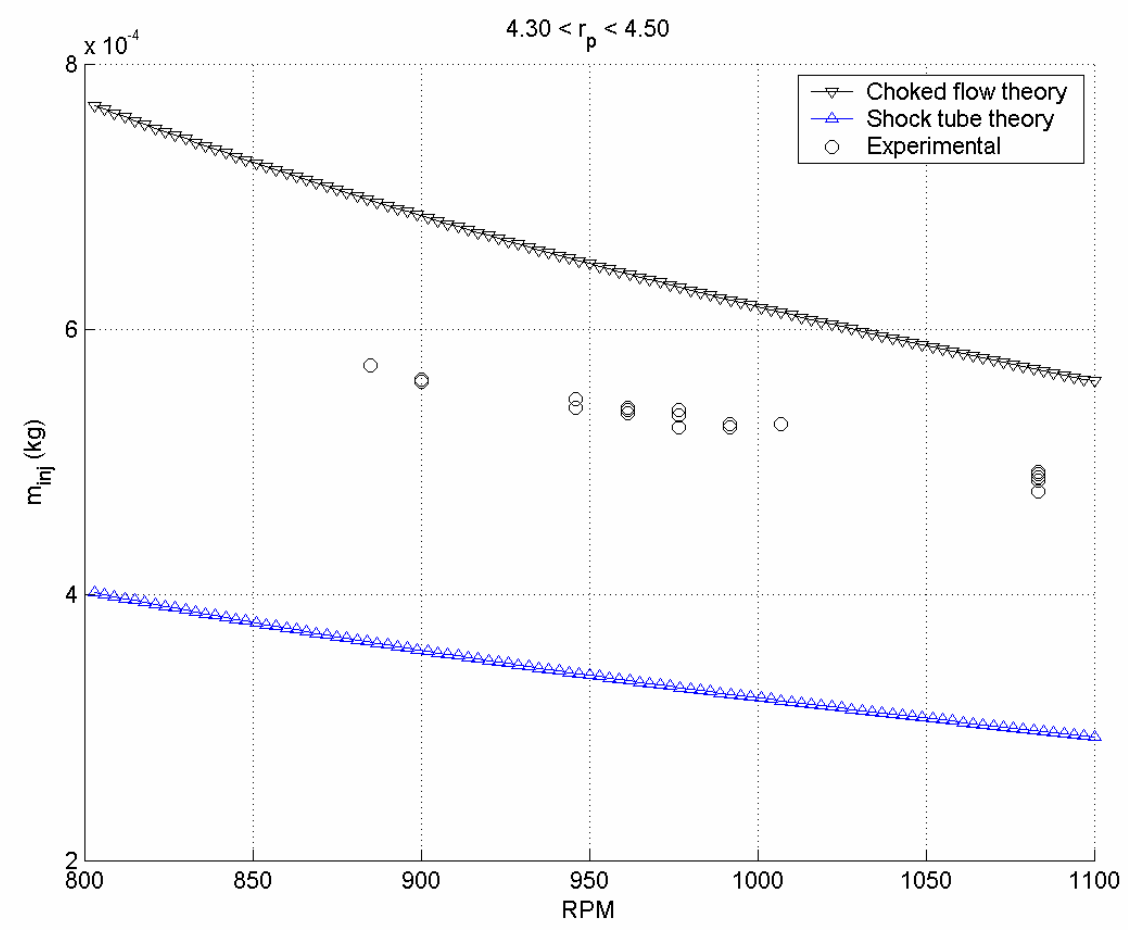

Figure 47: Injected mass from all test samples xx06 through xx10 ( $\left.\mathrm{p}_{\text {tank }} \approx 50 \mathrm{psig}\right)$.

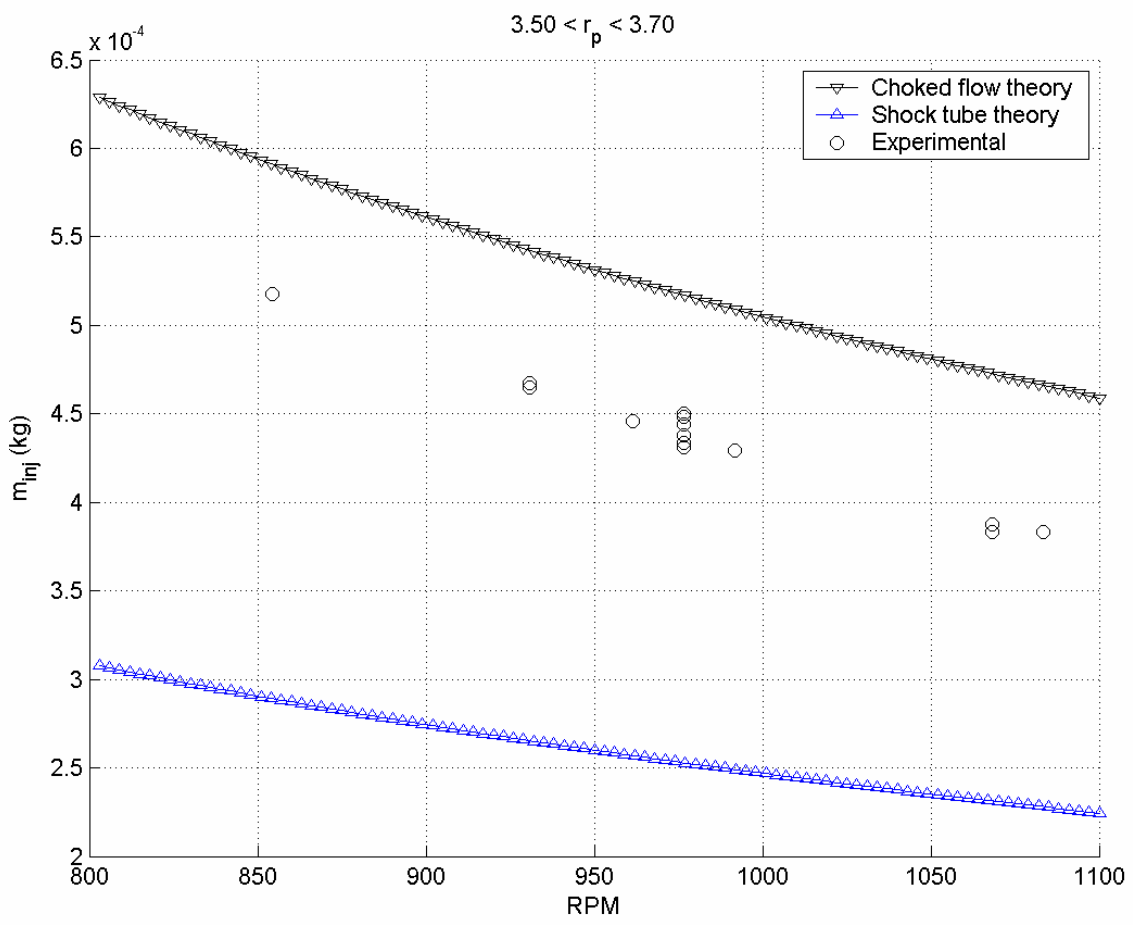

Figure 48: Injected mass from all test samples xx11 through xx15 ( $\left.\mathrm{p}_{\text {tank }} \approx 40 \mathrm{psig}\right)$. 


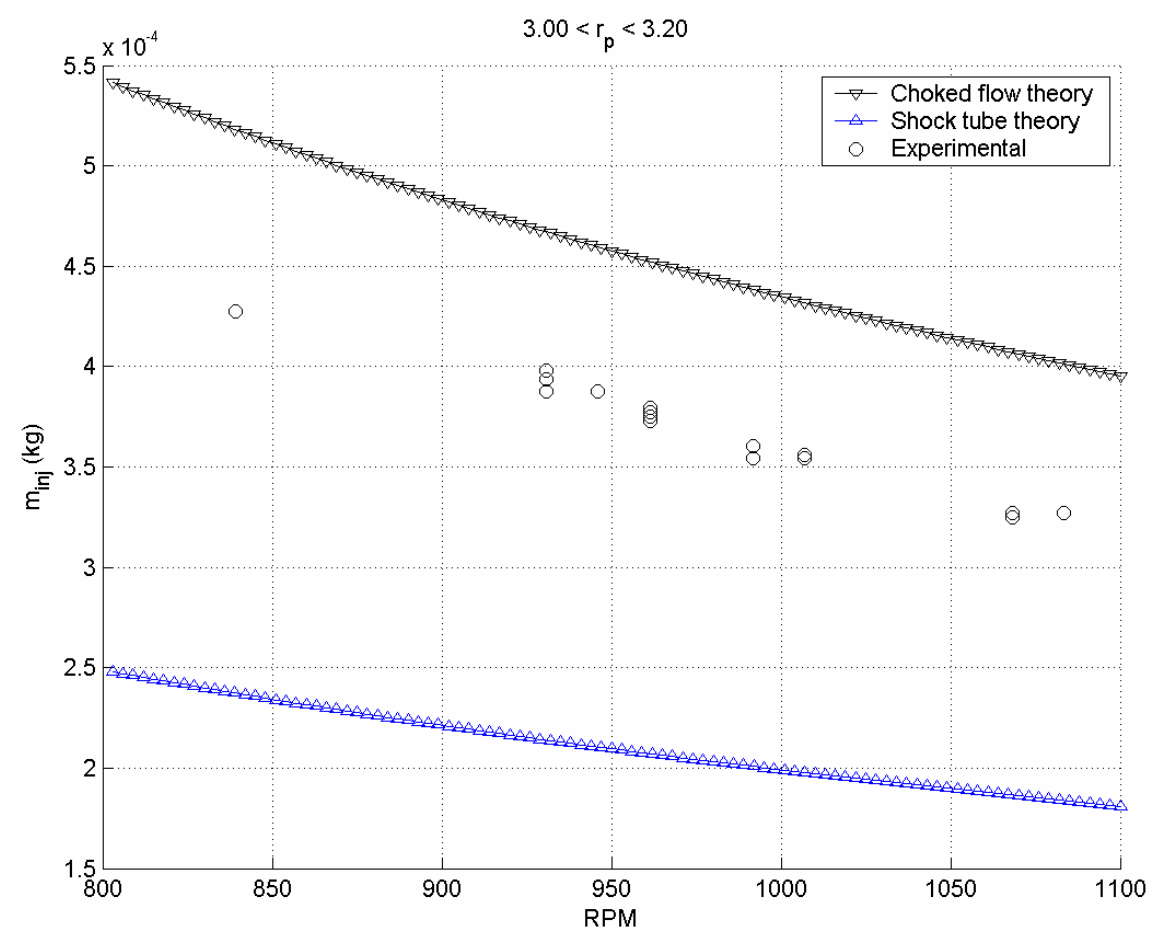

Figure 49: Injected mass from all test samples xx16 through $x \times 20\left(p_{\text {tank }} \approx 30 \mathrm{psig}\right)$.

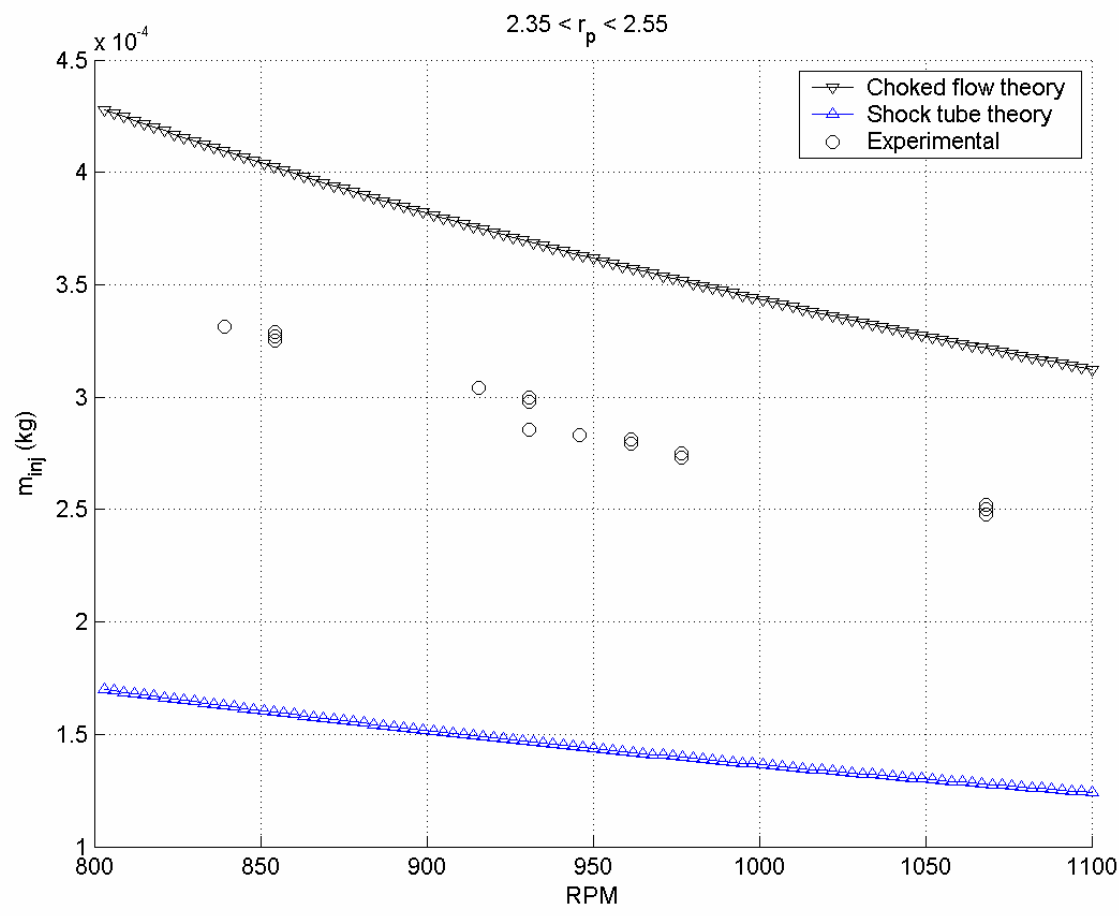

Figure 50: Injected mass from all test samples xx21 through $x \times 25\left(p_{\text {tank }} \approx 20 \mathrm{psig}\right)$. 


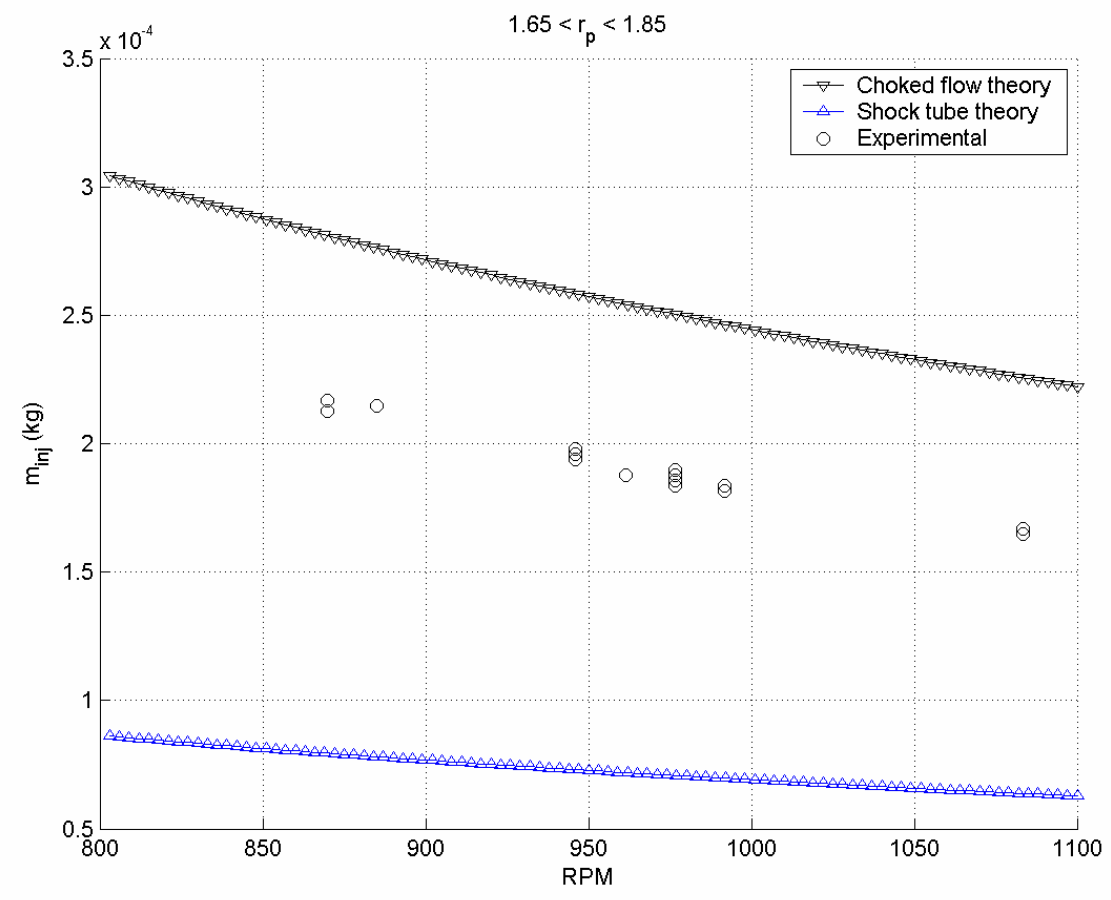

Figure 51: Injected mass from all test samples xx26 through xx30 ( $\left.\mathrm{p}_{\text {tank }} \approx 10 \mathrm{psig}\right)$.

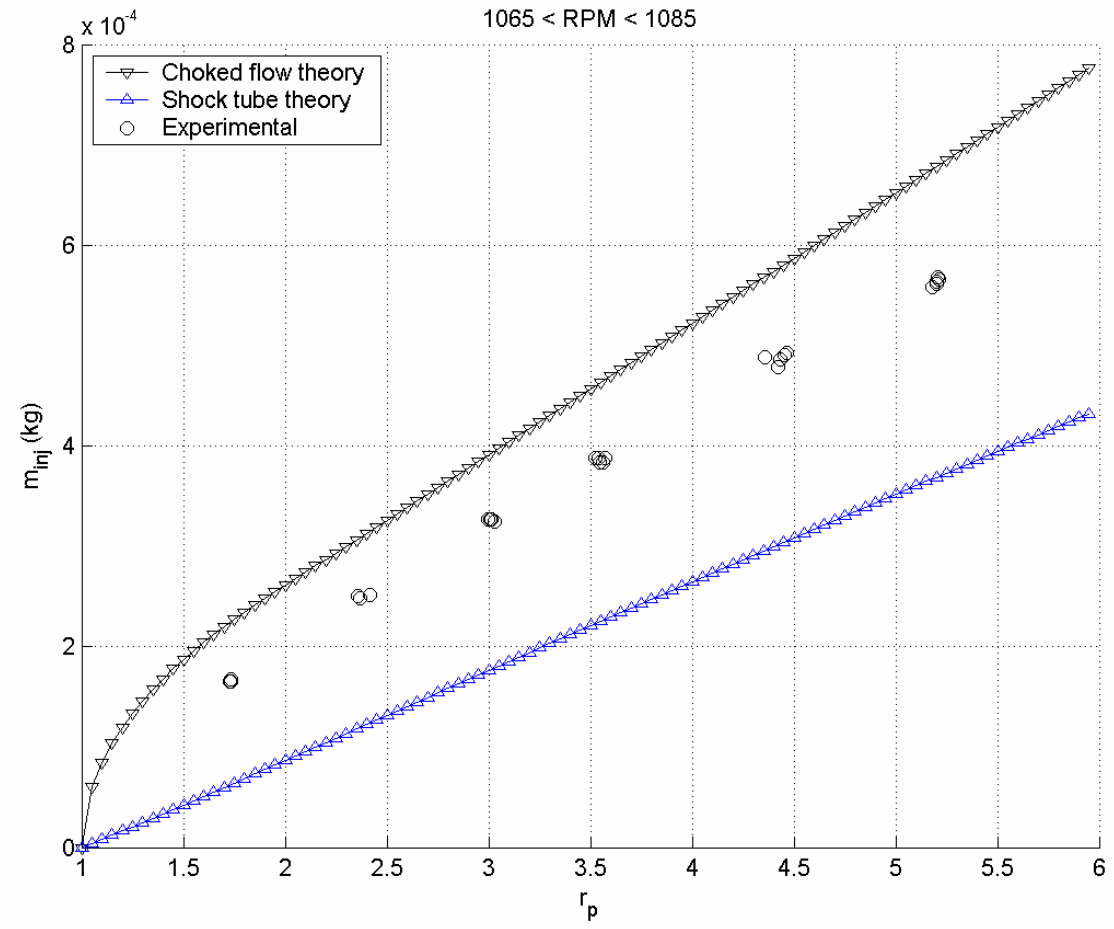

Figure 52: Injected mass from all test samples x201 through $x 230($ RPM $\approx 1075)$. 


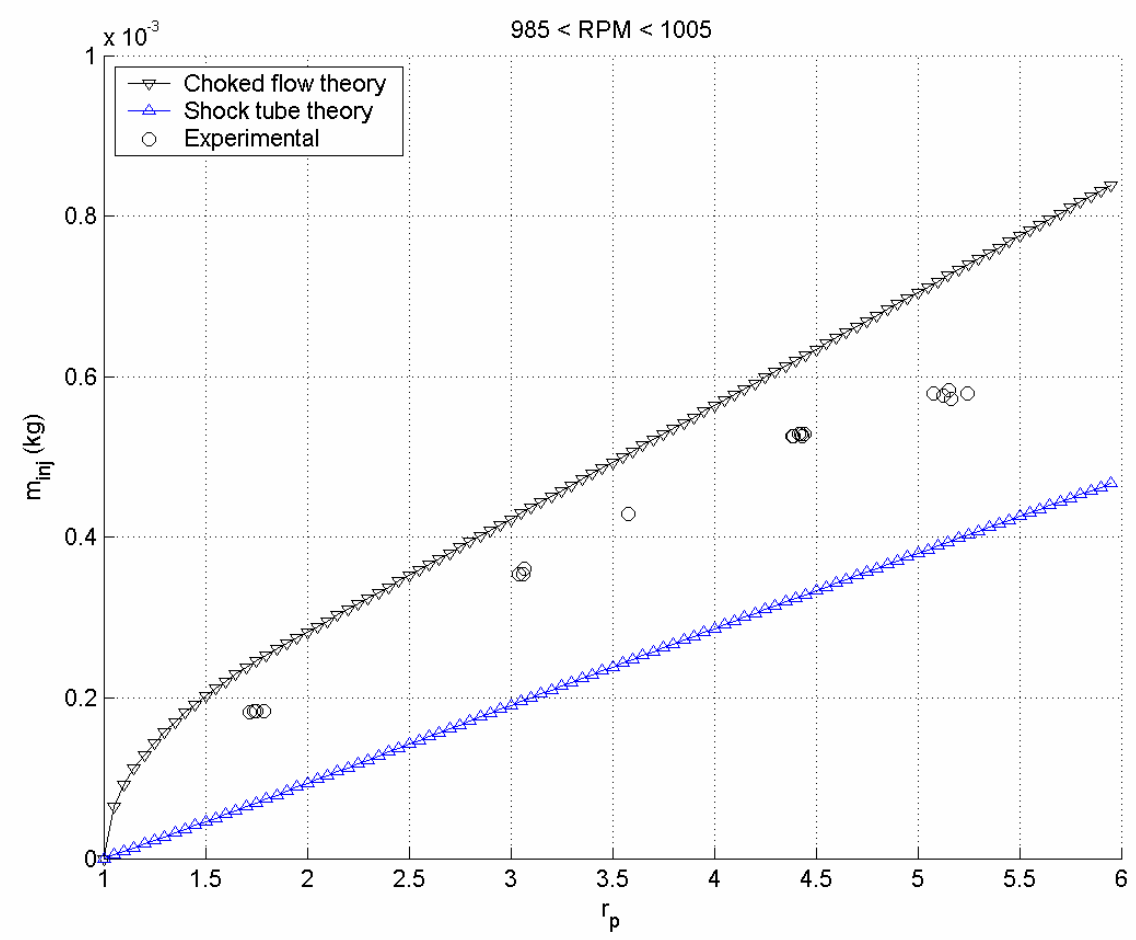

Figure 53: Injected mass from all test samples x301 through x330 (RPM $\approx 995)$.

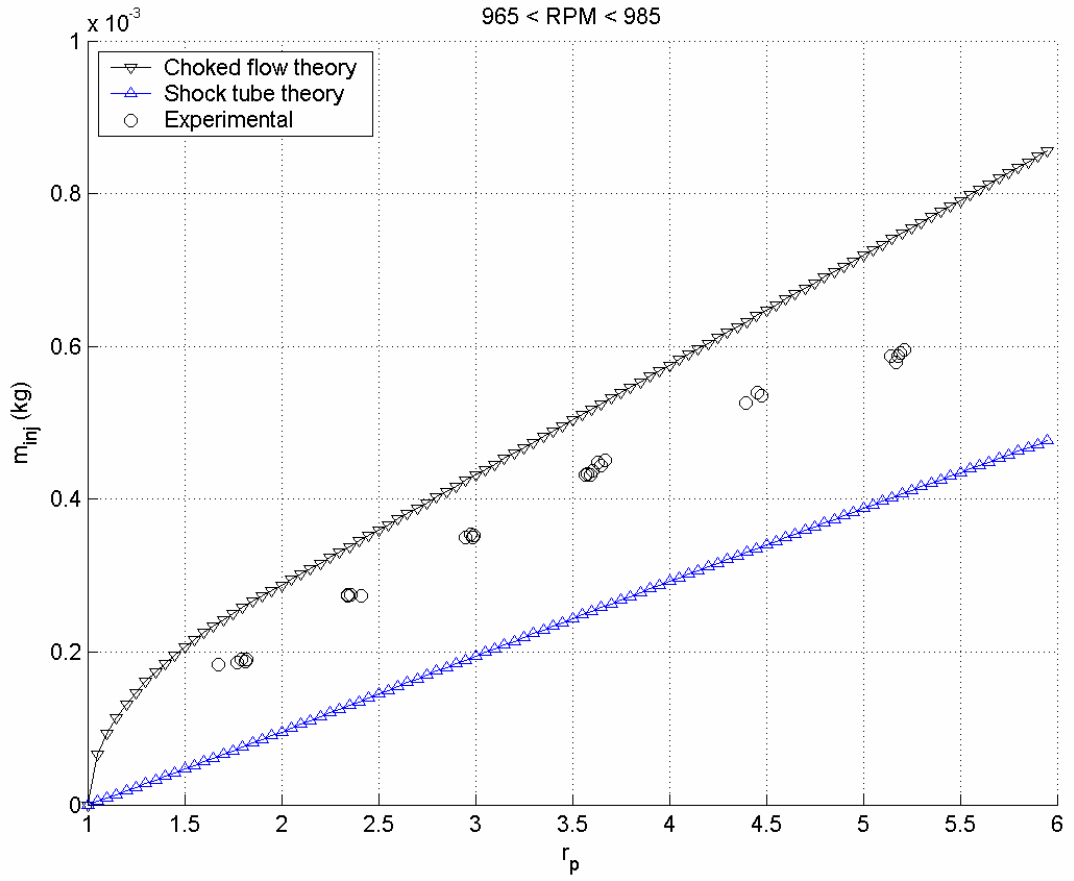

Figure 54: Injected mass from all test samples $x 401$ through $x 430($ RPM $\approx 975)$. 


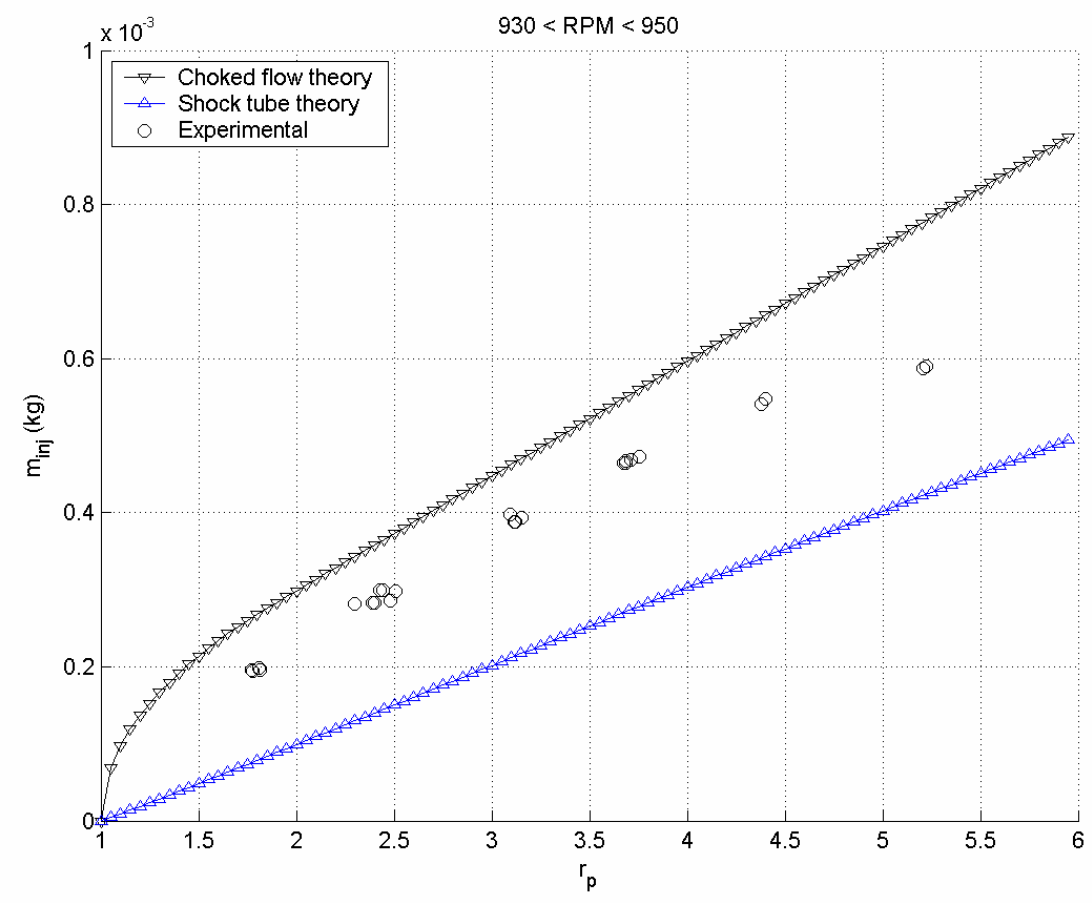

Figure 55: Injected mass from all test samples x501 through x530 (RPM $\approx 940)$.

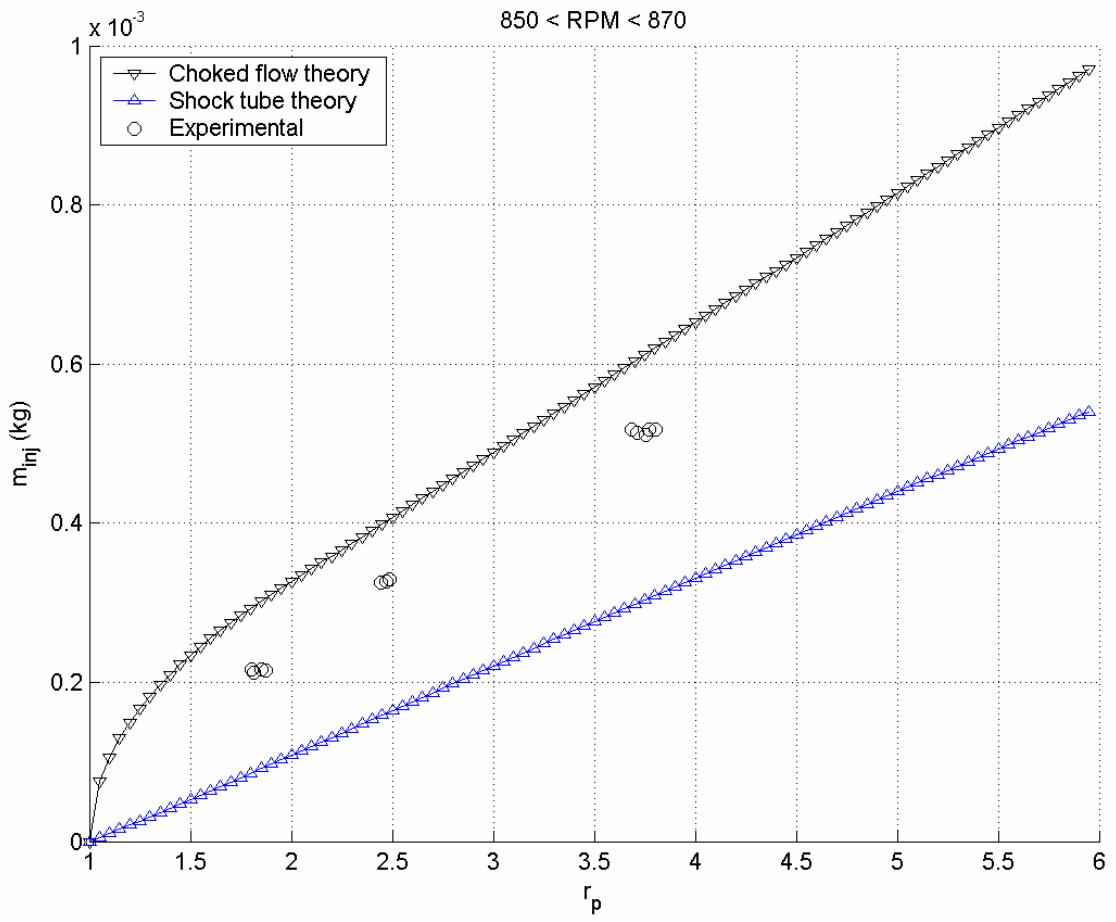

Figure 56: Injected mass from all test samples x601 through $x 630(\mathrm{RPM} \approx 860)$. 
Two important facts became evident from the collected data. First, qualitatively speaking, when considered as a function of valve speed and inlet-to-exhaust pressure ratio, the mass injected over a single $\mathrm{CCV}$ rotation behaved in a manner closely resembling trends predicted by choked flow theory. Second, a reduction in valve performance is clearly present and appears to be functionally dominated by the operating pressure ratio.

Data was collected and arranged using the Buckingham Pi theorem. By first suggesting that the total injected mass was a function of operating pressure upstream and downstream of the CCV as well as the CCV speed and open area, or

$$
m_{i n j}=f\left(p_{a}, p_{a f}, R P M, A_{C C V}\right),
$$

terms were grouped (and in some cases modified or redefined) so that two pi terms remained from the measurable quantities such that

$$
\Pi_{1}=\phi\left(\Pi_{2}\right)
$$

where

$$
\Pi_{1}=\frac{m_{i n j} \omega^{2}}{p_{a f} \sqrt{I}}, \quad \Pi_{2}=\frac{p_{a}}{p_{a f}}=r_{p}
$$

noting that

$$
I=\int_{\theta_{i}}^{\theta_{f}} A_{C C V} d \theta\left(m^{2}-\mathrm{rad}\right), \quad \omega=R P M\left(\frac{2 \pi}{60}\right)(\mathrm{rad} / \mathrm{sec}) .
$$

Integrating the open $\mathrm{CCV}$ area was solved numerically as a function of varying choke nozzle diameters (the ball geometry remained unchanged), and the resulting $5^{\text {th }}$ order curve fit equation was used in subsequent code to quickly determine its value. In early curve-fitting attempts, it was noted that a form of data spreading was occurring for 
larger values of $\Pi_{1}$ and $\Pi_{2}$ terms, so the injection mass carrying term was modified by another angular speed term to produce

$$
\frac{\Pi_{1}}{\omega}=\phi\left(\Pi_{2}\right),
$$

which correlated well to the pressure ratio term (Figure 57). A linear fit to the data yielded

$$
\frac{\Pi_{1}}{\omega}=1.702 E^{-5} \Pi_{2}-4.684 E^{-6},
$$

and demonstrated a goodness of fit of $\mathrm{R}^{2}=0.9969$.

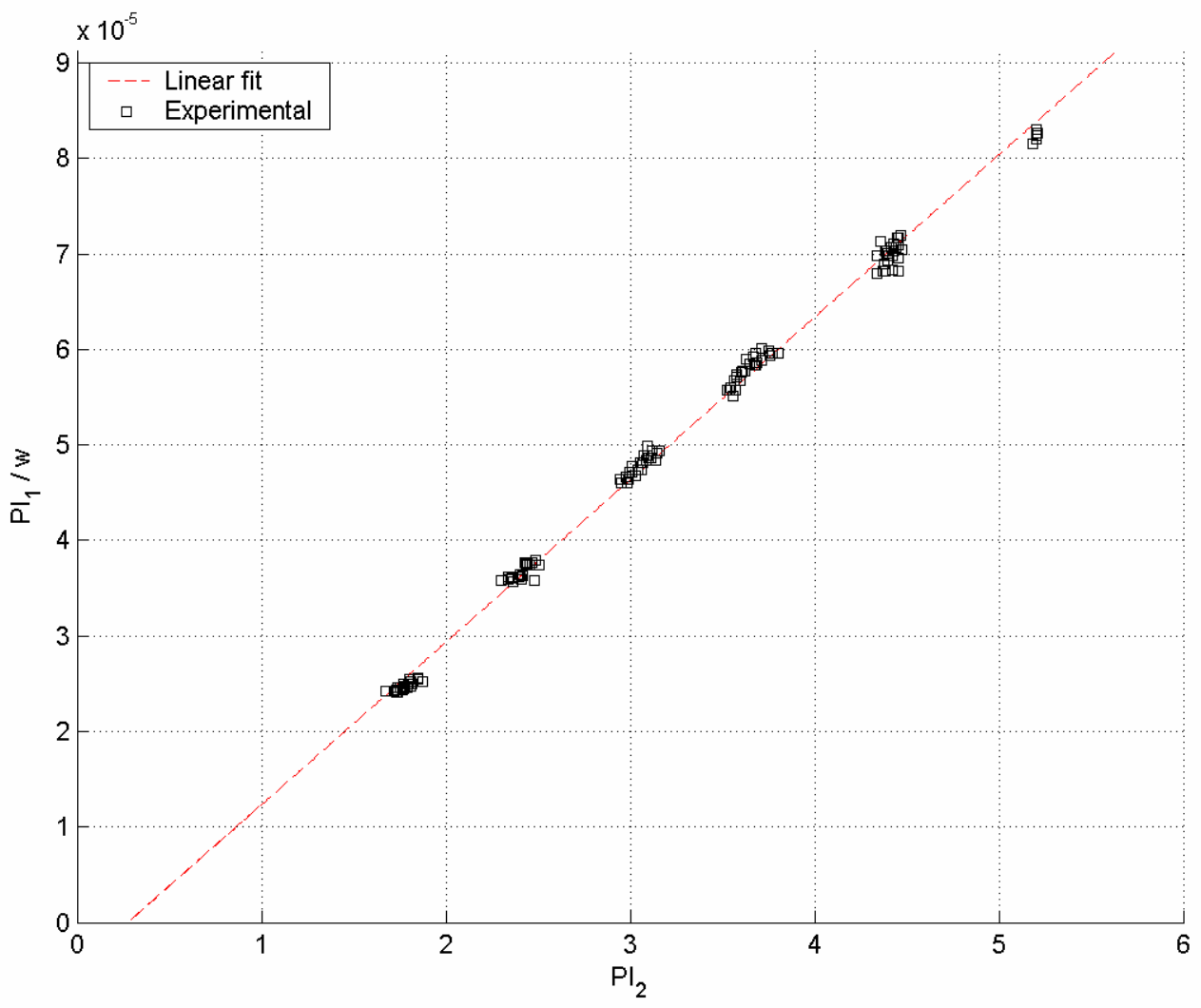

Figure 57: The modified injection mass pi term was highly correlated to the inter-CCV pressure ratio. 


\section{Constant Volume Results}

The constant volume experiments offered an opportunity to determine the instantaneous mass flow rate through the $\mathrm{CCV}$ as high pressure air was injected from one fixed volume $\forall_{l o}$ into another fixed volume, $\forall_{\text {hi }}$. Data subsets were compiled using a subset test matrix such as the one presented in Table 5. The subsets were then collected and used to determine the quasi-steady applicability of the results from the constant pressure tests.

Table 5: A test matrix was used for each constant volume test series " $x x^{\prime}$ in which instantaneous mass flow rate as a function of $\mathrm{CCV}$ speed, initial pressure ratios, and volume ratios was systematically investigated.

\begin{tabular}{|c|c|c|c|c|c|c|c|}
\hline \multirow{2}{*}{\multicolumn{2}{|c|}{$\begin{array}{l}V_{h i}=X \text { cc } \\
V_{10}=Y \text { cc }\end{array}$}} & \multicolumn{6}{|c|}{ High pressure (low volume) tank pressure (psig) } \\
\hline & & 60 & 50 & 40 & 30 & 20 & 10 \\
\hline \multirow{5}{*}{ 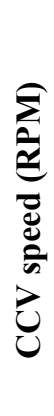 } & 1112 & $\mathrm{xx} 01-02$ & $\mathrm{xx} 11-12$ & $x \times 21-22$ & $\mathrm{xx} 31-32$ & $\mathrm{xx} 41-42$ & $\mathrm{xx} 51-52$ \\
\hline & 1034 & $\mathrm{xx} 03-04$ & $\mathrm{xx} 13-14$ & $\mathrm{xx} 23-24$ & $\mathrm{xx} 33-34$ & $\mathrm{xx} 43-44$ & $\mathrm{xx} 53-54$ \\
\hline & 995 & $\mathrm{xx} 05-06$ & $\mathrm{xx} 15-16$ & $\mathrm{x} \times 25-26$ & $x \times 35-36$ & $\mathrm{xx} 45-46$ & $\mathrm{xx} 55-56$ \\
\hline & 985 & $\mathrm{xx} 07-08$ & $\mathrm{xx} 17-18$ & $\mathrm{x} \times 27-28$ & $\mathrm{xx} 37-38$ & $\mathrm{xx} 47-48$ & $\mathrm{xx} 57-58$ \\
\hline & 933 & xx09-10 & xx19-20 & $\mathrm{xx} 29-30$ & xx39-40 & $x \times 49-50$ & $\times x 59-60$ \\
\hline
\end{tabular}

Simply plotting the data points gathered from one such test against theoretically determined values of transient pressure within the vessel (Figure 58) allowed an opportunity to determine in a qualitative sense the basic differences between a real CCV and one theoretically reversible CCV portrayed by choked flow theory. One difference, more quasi-steady in its general behavior, was the clear reduction in full-open pressure 
drop over an incremental time period, $\Delta \mathrm{t}$. In other words, the reduction in the time rate of change of pressure between the experimental data and theoretical predictions indicate a (constant) fractional drop in deliverable mass through the CCV over a select $\Delta \mathrm{t}$. The second difference, more transient in nature, appears to be rooted in the inertial effect of the fluid upon opening of the CCV. This more gradual rise to fully quasi-steady fluid movement is addressed in the literature [31], and was able to be modeled using an exponential operator.

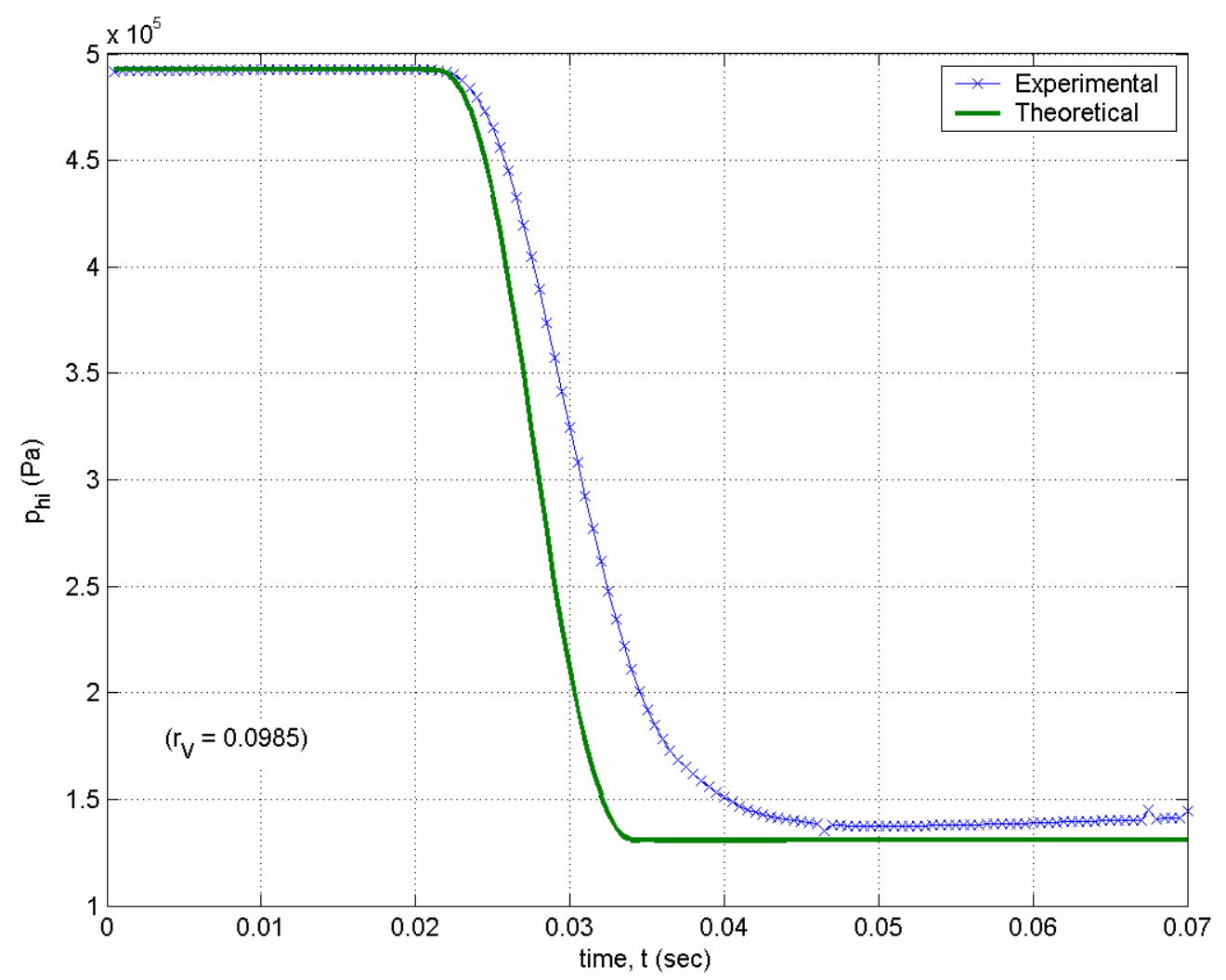

Figure 58: High frequency pressure transducer data was the primary source of experimental data during the constant volume experiments.

According to the earlier constant pressure tests, the total CCV mass flow for a single revolution correlated quite well with the pressure ratio, $r_{p}$. Applying this 
knowledge to the quasi-steady behavior of the $\mathrm{CCV}$ while addressing the need to exponentially increase from no-flow to full-flow within a particular time, resulted in the following instantaneous mass flow rate function:

$$
\dot{m}_{i} \approx \frac{\Delta m}{\Delta t}=z f\left(\dot{m}_{i, C F}\right)
$$

where

$$
z=\left(1-e^{t / \tau}\right), \quad \tau=\max \left[t_{S 1, R F L}, t_{R 1, R F L}\right]
$$

and the time the CCV has been open is given as t. Based upon results from test samples, the function, $\mathrm{f}$, acts as a dynamic (transient) discharge coefficient given by

$$
f=a \prod_{2}^{b} \omega^{c}, \quad a=0.0043, \quad b=0.65, \quad c=1.00 .
$$

Examples of the application of the empirical form are shown in Figure 59 and Figure 60.

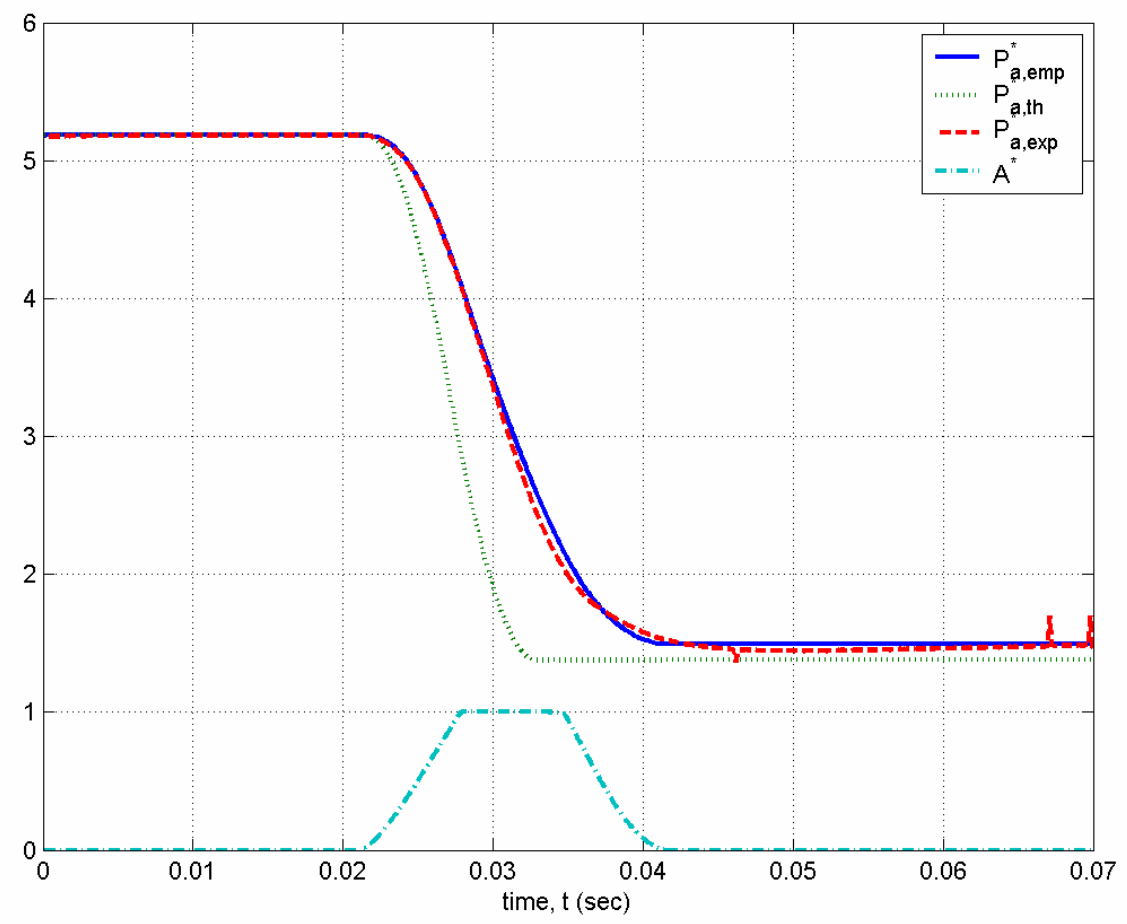

Figure 59: Experimental, theoretical, and empirical dimensionless results demonstrate the capability of the method to predict CCV injection behavior. 


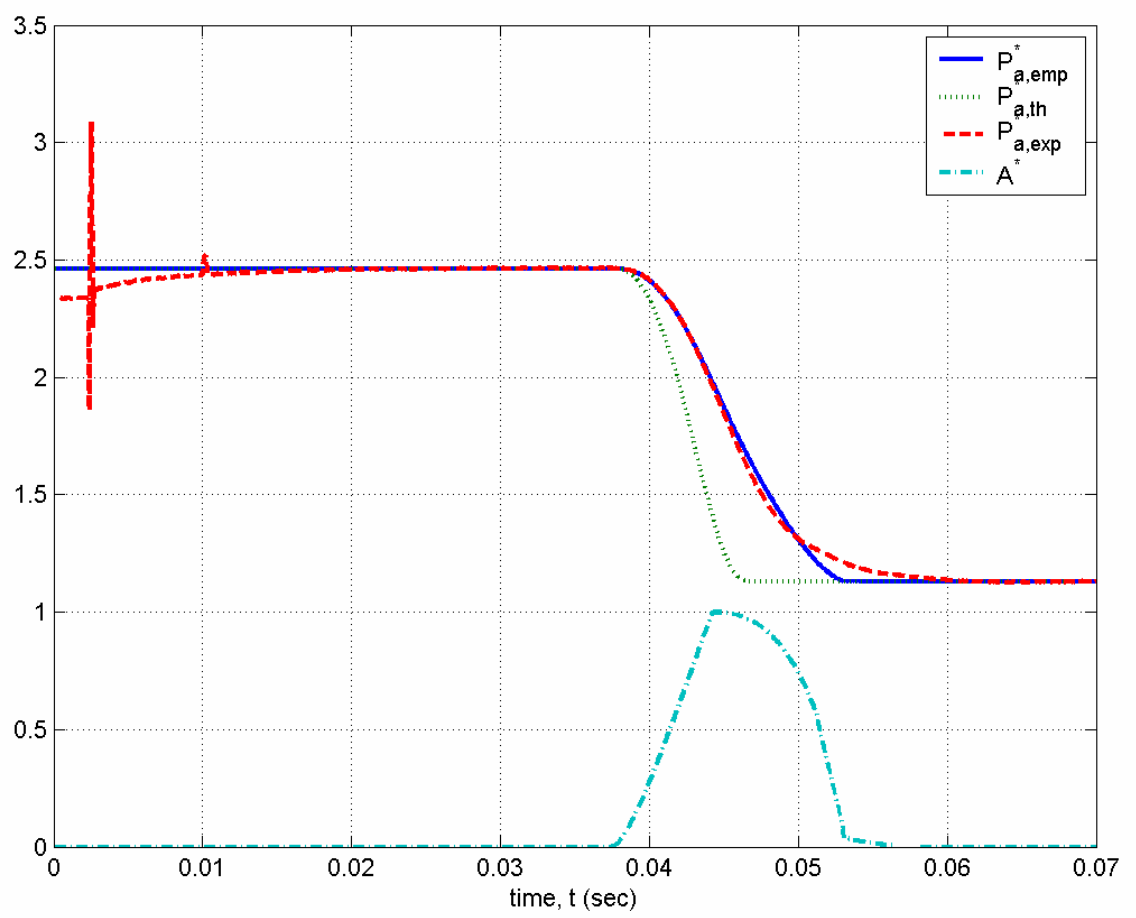

Figure 60: Lower values of total pressure ratios were equally well suited for the empirical equation because the form still used the choked flow notion of minimum area, $A^{*}$.

While perhaps not obvious, it is important to address the implications of the shaft speed term, $\omega$, in the empirical formula for instantaneous mass flow rate. It would seem that based upon the formula, discharge coefficients could conceivably go above what is theoretically possible by simply increasing the CCV shaft speed. This is not the case, however, because the transient term, $\mathrm{z}$, becomes more dominant with increasing speed in such a way that the discharge coefficient never reaches its full quasi-steady potential over the duration of time that the $\mathrm{CCV}$ is open. Thus, the dominating physics for the high speed case are transient and inertial, while the dominating physics in the low speed case are quasi-steady and viscous. 


\section{Quasi-Steady 1-D CIBAI Simulation Code Results}

A simulation code was written in Matlab and used to determine some of the general operating limits of a CIBAI engine. Particular emphasis was placed on the speed limit of such an engine. A code test matrix is shown below in Table 6, where the engine used was built on a pair of 30cc piston cylinder units attached head to head. The air-only side was set to a compression ratio of 30 , and the air-fuel side was kept at a "stock" compression ratio of 8 . The tendency of the peak temperature to drop with an increase in engine speed is a result primarily of the lack of time necessary to deliver enough energy into the air-fuel side to approach autoignition temperature range.

Table 6: Test results for a quasi-steady engine operating with fixed gear CCV may be capable of a tight range of potential auto ignition times.

\begin{tabular}{|c|c|c|}
\hline Speed & $\mathbf{T}_{\text {af,max }}$ & $\mathbf{C A}_{\text {Tmax }}$ \\
\hline $\mathbf{( R P M )}$ & $\mathbf{( K )}$ & $\mathbf{(}^{{ } \mathbf{B T D C})}$ \\
\hline 1800 & 807.31 & 3.05 \\
\hline 2400 & 811.22 & 2.65 \\
\hline 3000 & 817.22 & 2.25 \\
\hline 3600 & 822.07 & 1.7 \\
\hline 4200 & 824.44 & 1.05 \\
\hline 4800 & 824.96 & 0.6 \\
\hline 5400 & 824.34 & 0.25 \\
\hline
\end{tabular}

Figure 61 shows the fixed geometric evolution of the $\mathrm{CCV}$, and the simultaneous intakes and exhaust for the two cylinders. Figure 62 shows the resulting pressure trend as the engine is operated at a speed of 6600 RPM. The high compression ratio air-only side 
should produce as much as 116 atmospheres of pressure at TDC, but because of the opened $\mathrm{CCV}$, the peak is around 70 atmospheres instead. Figure 63 shows the temperature traces over the selected 3-cycle window, where it can be noted that the peak temperatures seem to still approach the range of autoignition condition. Figure 64 shows the mass as it moves into both cylinders during the induction stroke, then between the cylinders by way of the CCV near TDC, then finally exhausting after the power stroke. Figure 65 shows a trace of the total energy held within each cylinder. It is worth noting the rapidity with which the energy shifts into the air-fuel cylinder from the air-only side.

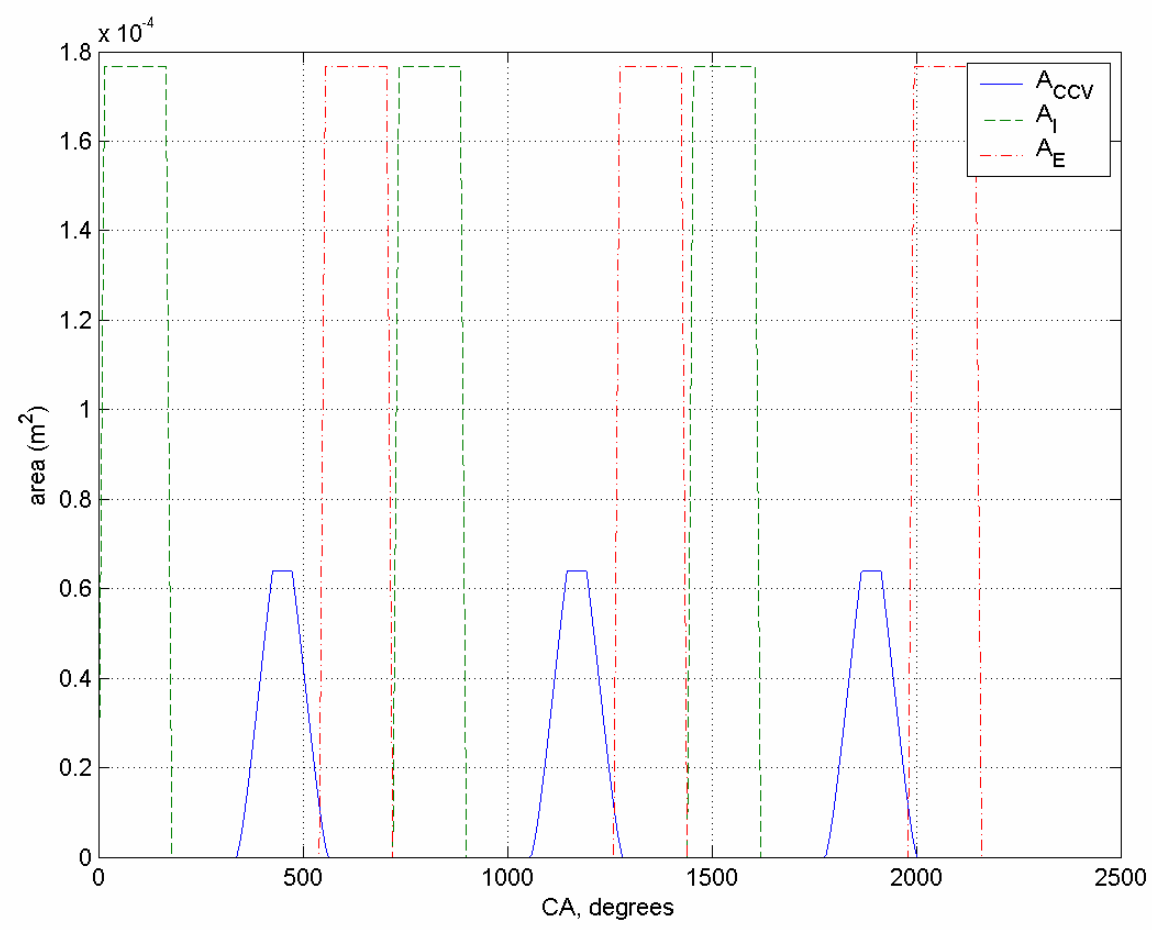

Figure 61: The fixed geometric behavior of the CCV, intake, and exhaust valves over 3 full cycles. 


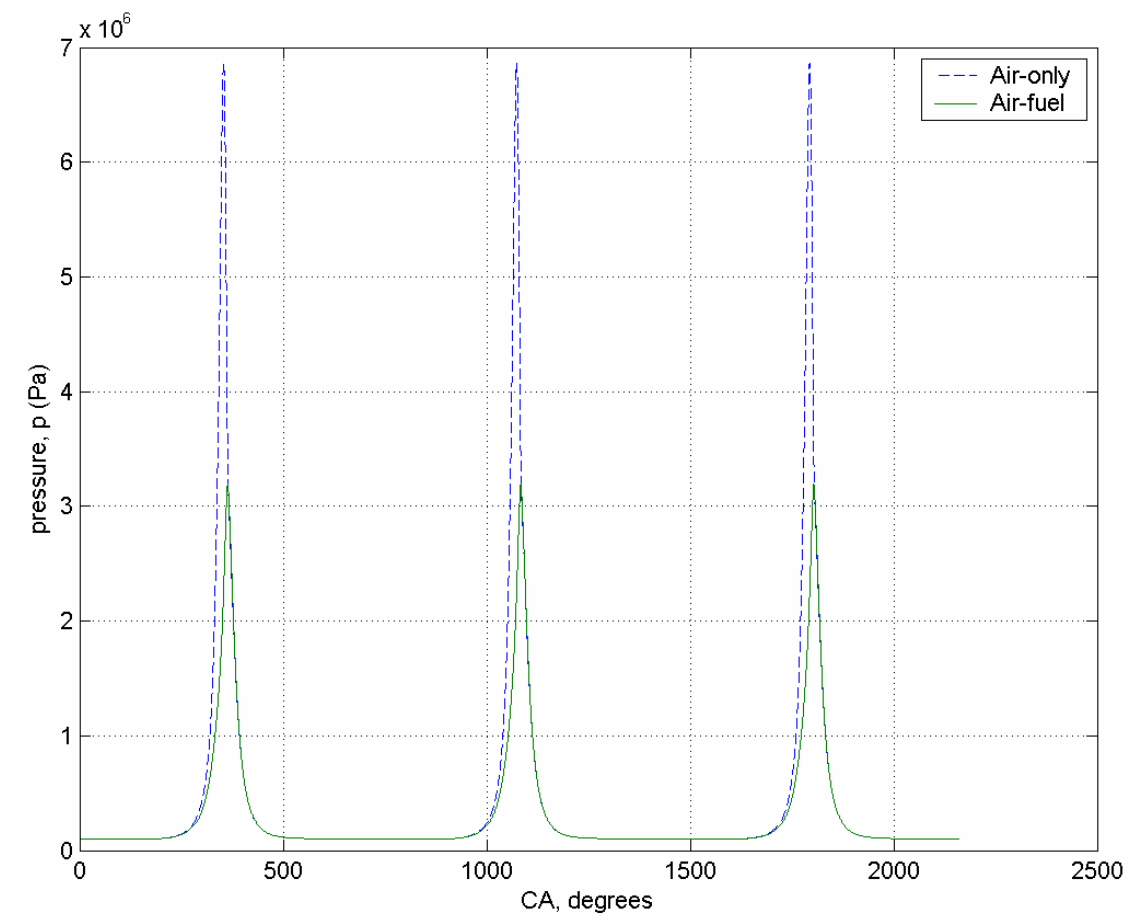

Figure 62: The pressure trends over 3 full cycles (6600 RPM).

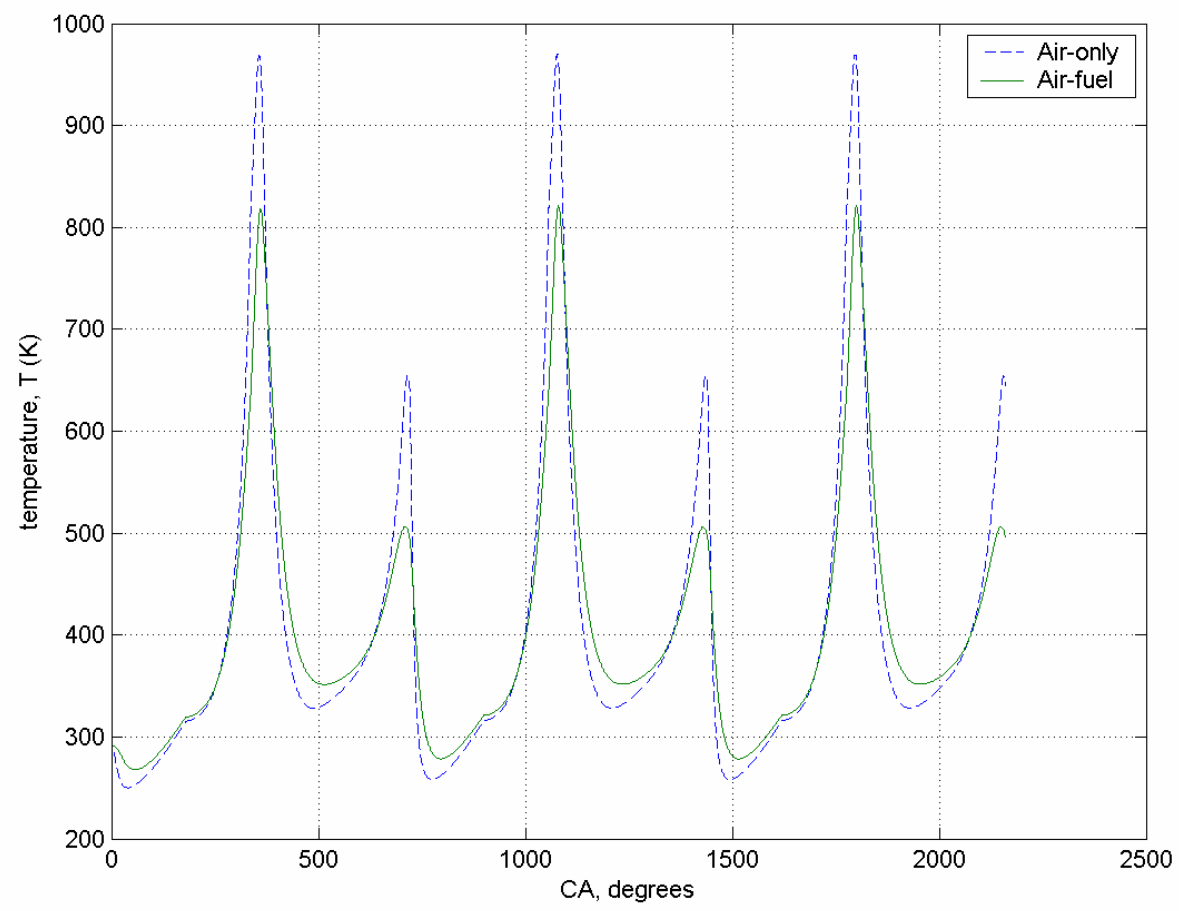

Figure 63: The temperature trends over 3 full cycles (6600 RPM). 


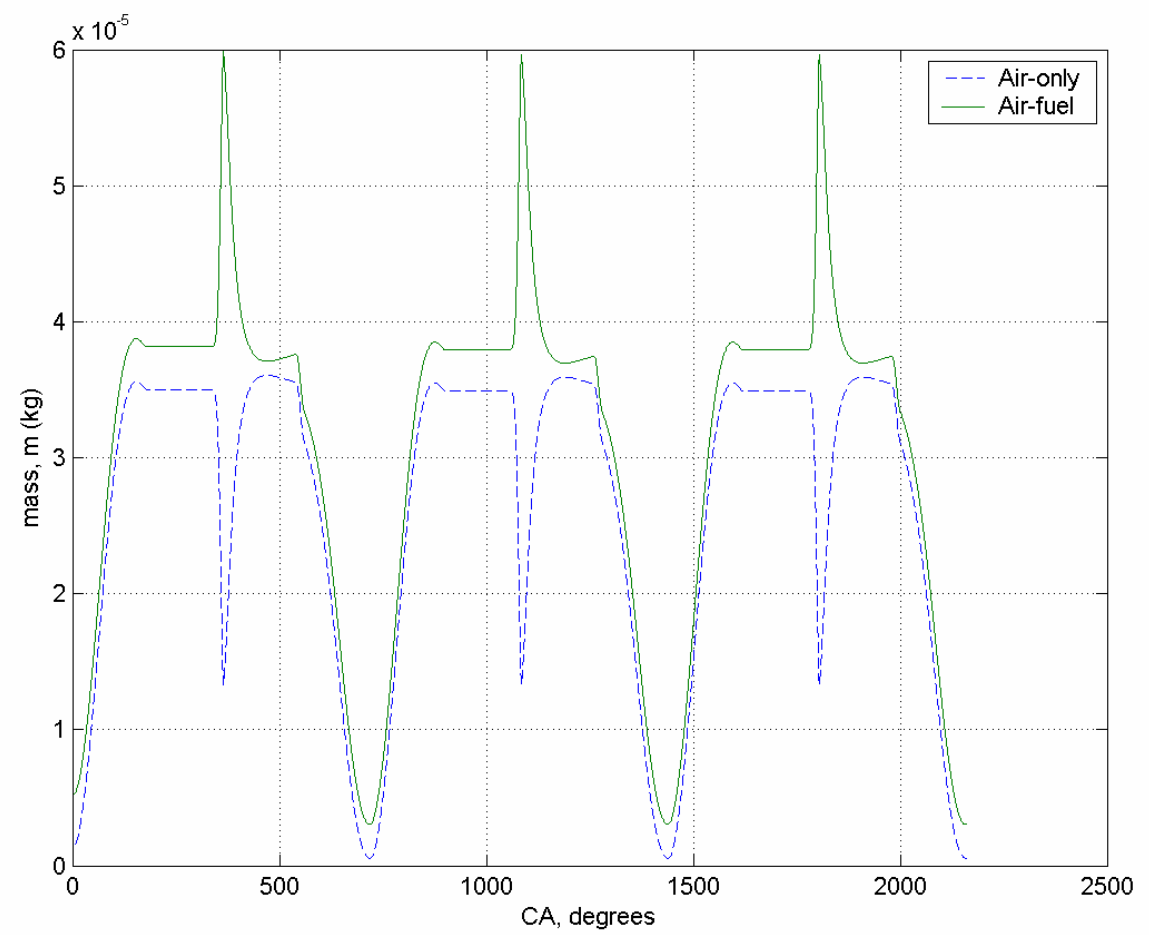

Figure 64: The mass trends over 3 full cycles (6600 RPM).

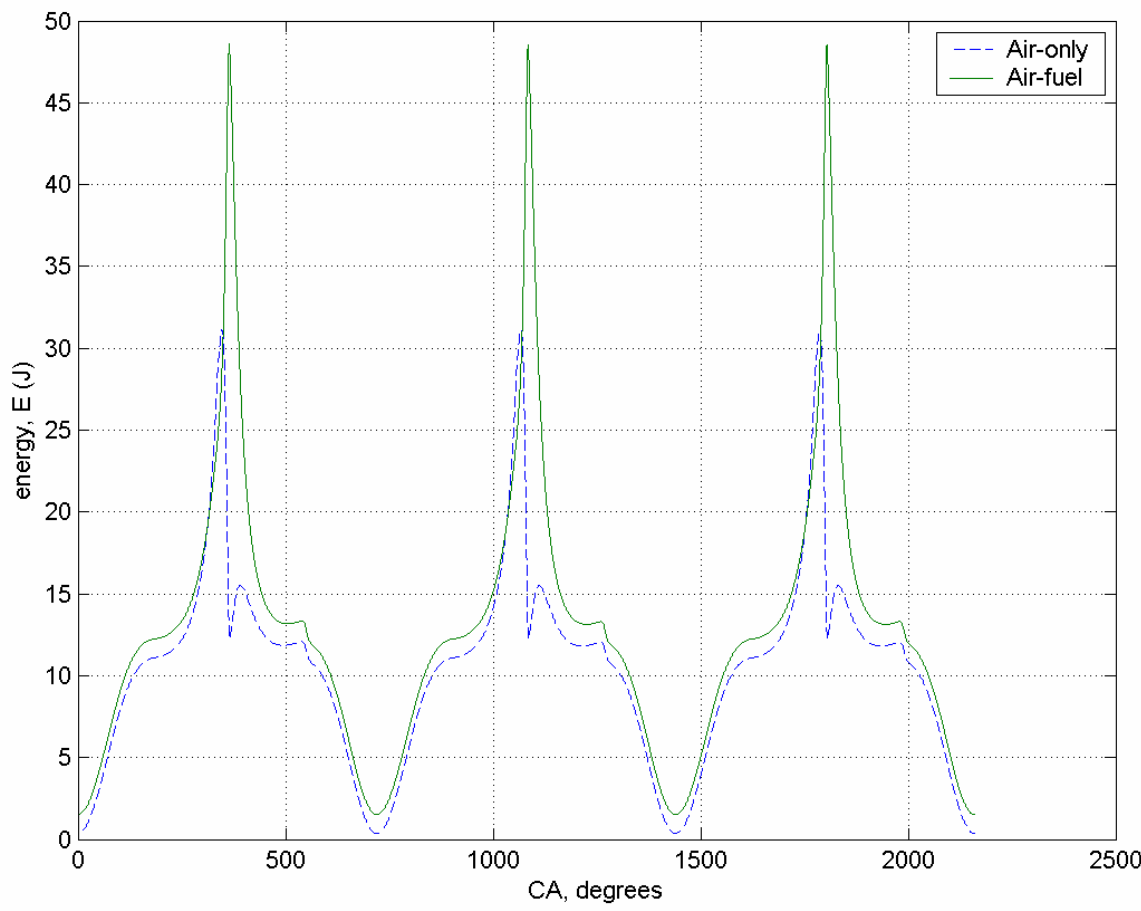

Figure 65: The energy trends over 3 full cycles (6600 RPM). 
Two potential geometric configurations were also considered based on available specifications for two different commercially available engines. One configuration was modeled based on paired 30cc engines, and the other was modeled based on paired 70cc engines. Figure 66 shows the 1-D code results for a paired set of $30 \mathrm{cc}$ engines mounted head to head, and Figure 67 shows results for the similar case using pared 70cc engines. In each case, a CCV inlet and exhaust channel length of four CCV ball diameters was used. These distances, in conjunction with the position of the air only and air fuel pistons at the moment of CCV opening, were used to determine the instantaneous shock-loss time lengths, $\mathrm{L}_{1}$ and $\mathrm{L}_{4}$. Potential autoignition within the air-fuel cylinder was indicated once the cylinder temperature exceeded $825 \mathrm{~K}$.

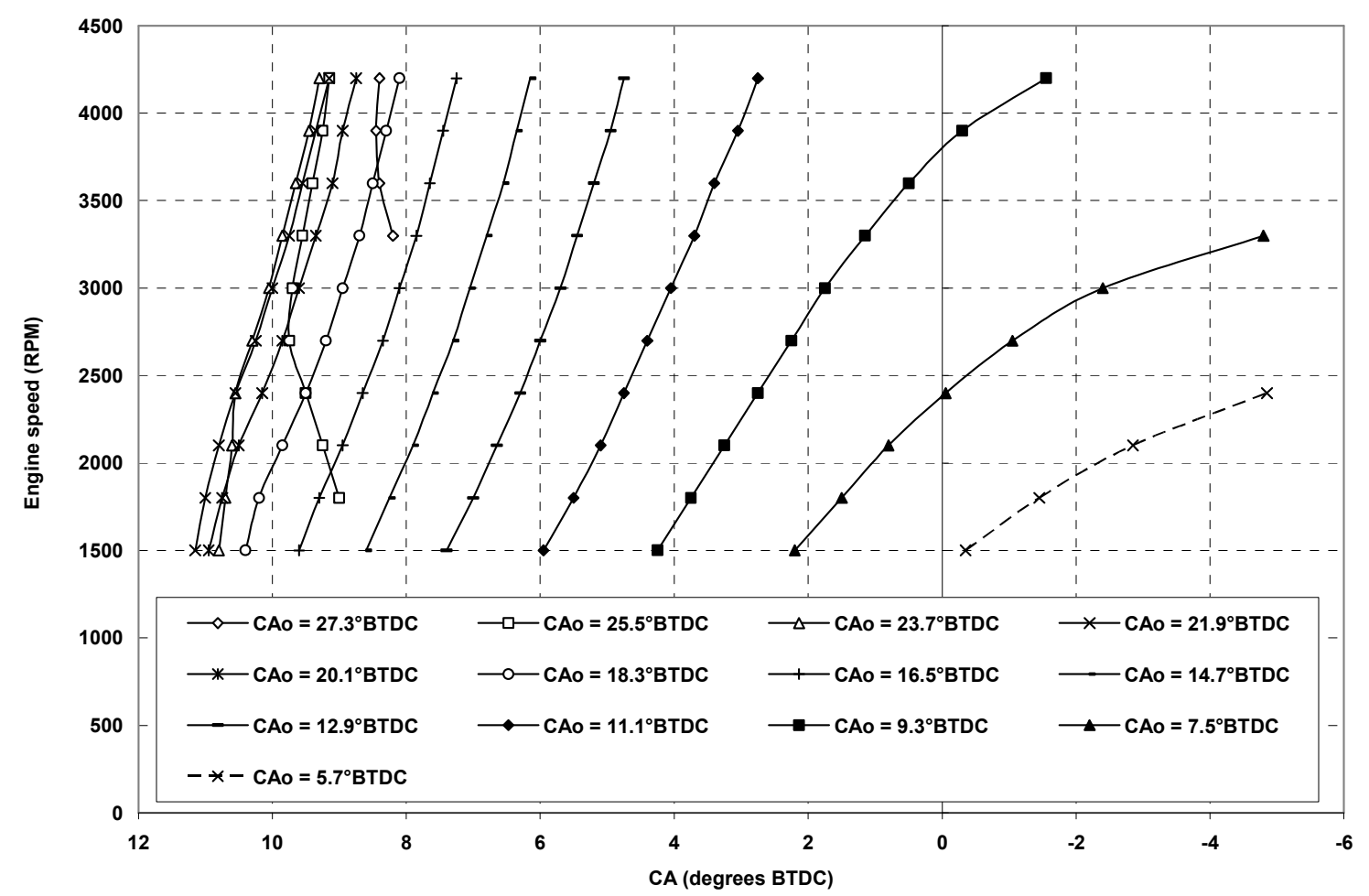

Figure 66: Potential autoignition for the paired set of 30cc engines in a CIBAI configuration. 


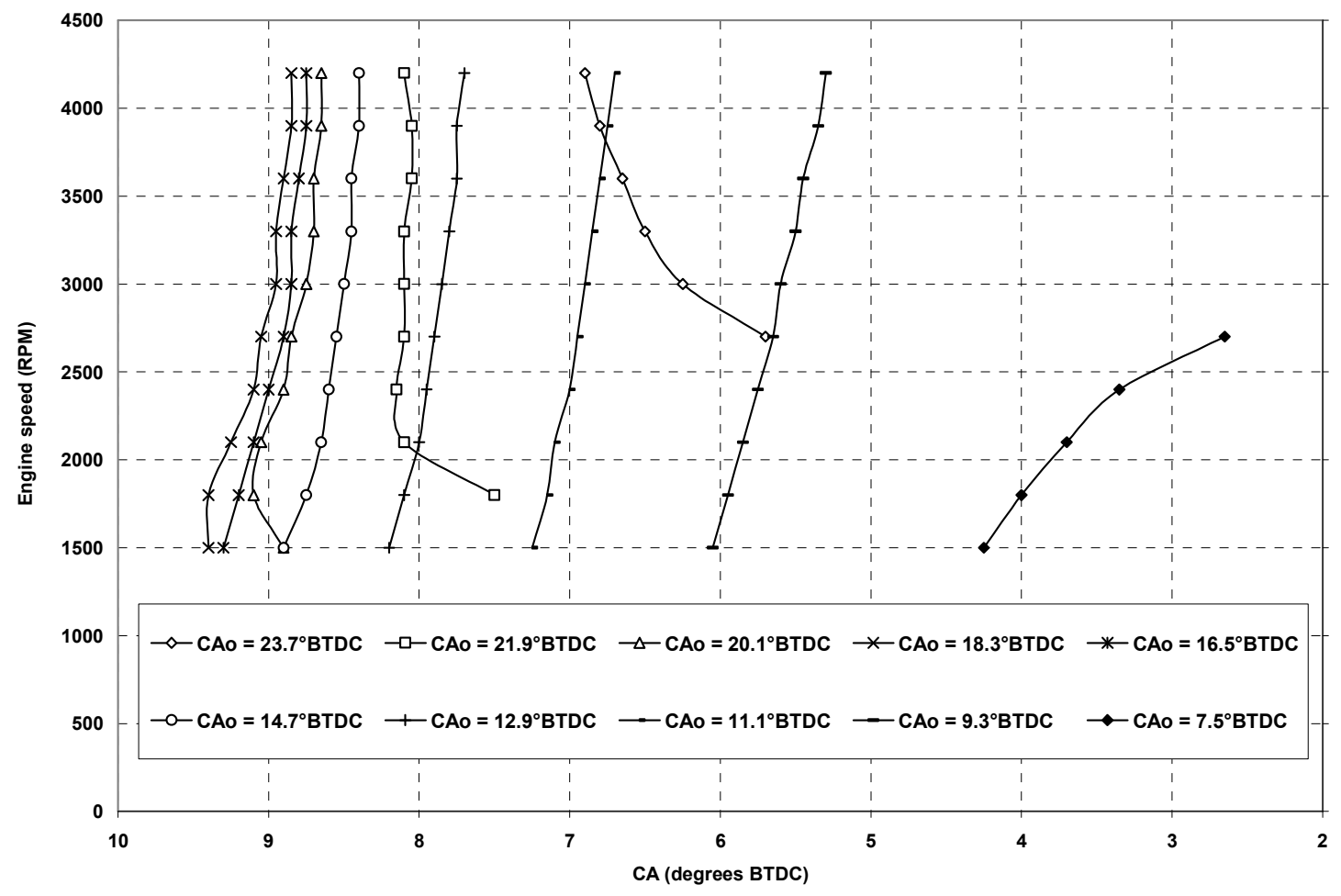

Figure 67: Potential autoignition for the paired set of 70cc engines in a CIBAI configuration. 


\section{Chapter 7: Conclusions}

The majority of this work faced the need to design, build, and test a valve for the purpose of application in a CIBAI engine. In light of these aims, the work was a success. The rotary ball valve design of the CCV tested offered many distinct advantages over a simple straight-hole ball valve, including the increased flexibility in outlet temporal evolution, the absence of "dead" volume that would be encountered with a straight-hole design, and the basic simplicity of single-opening-per-revolution operation. The finished $\mathrm{CCV}$ was successful in sealing against relatively high pressures $(>120$ psia) while simultaneously being capable of rapid acceleration through a single revolution within a few milliseconds without significant friction or loss of speed $(930<\mathrm{RPM}<1130)$.

Testing of the CCV proved enlightening in many different respects. High frequency acoustic and wave data proved indispensable in describing the rapid transient behavior of gas subjected to CCV ball operation, specifically locating the onset of Mach waves, intense acoustic wave packets, and even residual Helmholtz resonance within the tube. It was also especially useful in determining the overall applicability of shock tube theory to the transient gas dynamic behavior, proving in particular that the "rough" nature and turning angle of the ball prevented the passage of significant normal shock waves through the system. Further evaluation of the gas flow in terms of overall mass throughput per cycle demonstrated the average loss of the CCV when compared to ideal gas dynamic models such as choked compressible flow. Additional constant volume experiments proved extremely useful in the application of the experimentally derived mass flow modifications to rapidly changing closed systems which better simulate engine cylinders. 
Finally, the re-introduction of an empirical CCV mass flow rate model, namely

$$
\dot{m}_{\text {emp }}=z f \dot{m}_{\text {ideal }},
$$

where

$$
z=\left(1-e^{t / \tau}\right), \quad f=0.0043 \omega\left(\frac{p_{h i}}{p_{l o}}\right)^{0.65},
$$

into specialized 1-D quasi-steady model showed some likely ranges of successful cyclic autoignition capabilities in an actual CIBAI engine. Use of commercially available 30cc engines coupled head-to-head in a CIBAI arrangement operating at no load between 1500 and 4250 RPM can be facilitated at initial CCV opening crank angle positions between 27.3 and $5.7{ }^{\circ}$ BTDC. Larger displacement arrangements, such as the $70 \mathrm{cc}$ version tested demonstrated a reduction in CCV opening position range with values between 23.7 and 7.5 degrees ${ }^{\circ} \mathrm{BTDC}$. It was also apparent that particular initial injection timing plays a critical role in the variation of potential autoignition times with respect to engine speed. For example, using the 30cc-based model operating from 1500 to 4250 RPM, beginning injection at $23.7^{\circ} \mathrm{BTDC}$ showed an indicated autoignition time range of 10.8 to 9.3 ${ }^{\circ}$ BTDC. Keeping all other conditions the same and adjusting the beginning injection time to $9.3{ }^{\circ} \mathrm{BTDC}$ demonstrated a much wider indicated autoignition time range of 4.25 to $-1.55^{\circ} \mathrm{BTDC}$. 


\section{Chapter 8: Recommendations}

It is the hope and intent of the researcher that the information contained in this work is extended to future work realizing the CIBAI engine. Based on the heat, sealing and friction demands that will be applied to an actual CIBAI CCV, it seems most likely that the successful design must incorporate major components made of a high grade modern ceramic material. Although the modified ball type design has shown that it can be used effectively in a CIBAI design, perhaps a seated thimble shaped design that could allow a more gentle turn through the $\mathrm{CCV}$ would alleviate some mass flow rate reduction that no doubt corresponded to the sharp turn angle of the CCV tested. One additional option might be to use a spring and cam mechanism to apply pressure to the seated valve body, and then release pressure on the body when it is in the open position thus reducing overall friction and wear.

Potential future research exists in the notion of a combustion bomb study in which even a low temperature CCV such as the one used in this work could be used on a "disposable seal" type basis. The components used for the herein contained research to cause the single rapid injection event might even be reused for this purpose in the future. It would seem that the results of such single-injection combustion bomb research would be able to firmly establish the required chemical and thermal unknowns such as fuel types, stoichiometries, and transient thermal effects on CCV behavior. 


\section{References}

1. Echavarria, F. Ignition by Air Injection (CIBAI) for Controlled Auto-Ignition in a CFR Engine. PhD Dissertation, West Virginia University CEMR, 2006.

2. Heywood, J. B., Internal Combustion Engine Fundamentals, McGraw-Hill Inc., New York 1988.

3. Kuo, K.K., Principles of Combustion, John Wiley \& Sons, Inc., New York 1986.

4. Young, D. R., Munson, B. R., and Okiishi, T.H., A Brief Introduction to Fluid Mechanics, $2^{\text {nd }}$ Ed., John Wiley \& Sons, Inc., New York 2001.

5. Anderson, J. D. jr., Fundamentals of Aerodynamics, $3{ }^{\text {rd }}$ Ed., McGraw-Hill, Inc., New York 2001.

6. Loth, J. L., and Morris, G. J. Compression Ignition Engine by Air Injection from AirOnly Cylinder to Adjacent Air-Fuel Cylinder. US Patent No.: 6.994.057, February 7, 2006.

7. Loth, J. L. Compression Ignition By Air Injection (CIBAI) Cycle and Engine. US Patent No.: 6.899.061, May 31, 2005.

8. Flameless Combustion By: Bob Brooks, Advanstar Communications, Inc. Motor Age, October 2007.

9. Gasoline-diesel combo moves closer to reality. By: Weernink, Wim Oude, Automotive News, 00051551, 1/7/2008, Vol. 82, Issue 6289

10. Is yesterday's problem tomorrow's solution? By: Sherman, D., Automotive News, 00051551, 11/19/2007, Vol. 82, Issue 6282. 
11. Zhao, H., Peng, Z., and Ladommatos, N. Understanding of controlled autoignition combustion in a four-stroke gasoline engine. Proc. Instn Mech. Engrs, Part D: Journal of Automobile Engineering, 2001, 215.

12. Atkins, M. J., and Koch, C. R. The effect of fuel octane and dilutent on homogeneous charge compression ignition combustion. Proc. Instn Mech. Engrs, Part D: Journal of Automobile Engineering, 2004.

13. Bengtsson, J., Strandh, P., Johansson, R., Tunestal, P., and Johansson, B. Hybrid modeling of homogeneous charge compression ignition (HCCI) engine dynamics- a survey. International Journal of Control, 2007, 80.

14. Bengtsson, J., Strandh, P., Johansson, R., Tunestal, P., and Johansson, B. Hybrid control of homogeneous charge compression ignition (HCCI) engine dynamics. International Journal of Control, 2006, 79.

15. Xu, H., Wyszynski, M., Megaritis, A., Yap, D., Wilson, T., Qiao, J., Richardson, S., Golunski, S., Peucheret, S. Research on expansion of operating windows of controlled homogeneous auto-ignition engines. International Journal of Engine Research, 2007, 8 .

16. Yang, J. Expanding the operating range of homogeneous charge compression ignition-spark ignition dual-mode engines in the homogeneous charge compression ignition mode. International Journal of Engine Research, 2005, Special Issue Paper.

17. Jia, M., and Xie, M. Numerical solution of homogeneous charge compression ignition combustion using a multi-dimensional model. Proc. Instn Mech. Engrs, Part D: Journal of Automobile Engineering, 2007, 221. 
18. Cao, L., Zhao, H., Jiang, X., Kalian, N. Understanding the influence of valve timings on controlled autoignition combustion in a four-stroke port fuel injection engine. Proc. Instn Mech. Engrs, Part D: Journal of Automobile Engineering, 2005.

19. Mahrous, A., Wyszynski, M., Wilson, T., Xu, H. Computational fluid dynamics simulation of in-cylinder flows in a motored homogeneous charge compression ignition engine cylinder with variable negative valve overlapping. Proc. Instn Mech. Engrs, Part D: Journal of Automobile Engineering, 2007, 221.

20. Angelos, J., Puignou, M., Andraea, M., Cheng, W., Green, W., and Singer, M. Detailed chemical kinetic simulations of homogeneous charge compression ignition engine transients. International Journal of Engine Research, 2007, 9.

21. Kalghatgi, G., and Head, R. Combustion limits and efficiency in a homogeneous charge compression ignition engine. International Journal of Engine Research, 2006, 7.

22. Idris, A., and Pullen, K. Correlations for the discharge coefficient of rotating orifices based on the incidence angle. Proc. Instn Mech. Engrs, Part A: Journal of Power and Energy, 2005, 219.

23. Idris, A., Pullen, K.,, and Barnes, D. An investigation into the flow within inclined rotating orifices and the influence of incidence angle on the discharge coefficient. Proc. Instn Mech. Engrs, Part A: Journal of Power and Energy, 2004, 218.

24. Gault, R., Thornhill, D., and Fleck, R. Alternative method to evaluate discharge coefficients. Part 1: feasibility study. Proc. Instn Mech. Engrs, Part C: Journal of Mechanical Engineering Sciences, 2007, 221 
25. Zhang, Z. New method for comparing flow in spring-loaded valves. Proc. Instn Mech. Engrs, Part A: Journal of Power and Energy, 2006, 220.

26. Kojima, E., Yamazaki, T., and Shinada, M. Development of a New Simulation Technique Based on the Modal Approximation for Fluid Transients in Complex Pipeline Systems with Time-Variant Nonlinear Boundary Conditions. ASME Journal of Fluids Engineering, 2007, 129.

27. Thornhill, D., Li, H., Fleck, R., and Cuningham, G. Modeling of unsteady gasdynamic flow in a pipe and at its exit using CFD. Proc. Instn Mech. Engrs, Part D: Journal of Automobile Engineering, 2006, 220.

28. Pavelyev, A., Reshmin, A., Teplovodskii, S., Fedoseev, S. On the Lower Critical Reynolds Number for Flow in a Circular Pipe. Fluid Dynamics, 2003, 4.

29. Cunningham, G., Kee, R., Kenny, R. Reed valve modeling in a computational fluid dynamics simulation of the two-stroke engine. Proc. Instn Mech. Engrs, Part D: Journal of Automobile Engineering, 1999, 213.

30. Francis, J., and Betts, P. Modeling incompressible flow in a pressure relief valve. Proc. Instn Mech. Engrs, Part E: Journal of Process Mechanical Engineering, 1997, 211.

31. Arkhipov, V., Berezikov, A., and Trofimov, V. On the Quasi-Steadiness Hypothesis as Applied to Gas Exhaustion from a Receiver. Journal of Applied Mechanics and Technical Physics, 2004, 45.

32. Yoon, J., Sung, N., and Lee, C. Discharge coefficient equation of a segmental wedge flowmeter. Proc. Instn Mech. Engrs, Part E: Journal of Process Mechanical Engineering, 2008, 222. 
33. Smythe, K., and Bryner, N. Short-Duration Autoignition Temperature measurements for Hydrocarbon Fuels Near Heated Metal Surfaces. Combustion Science and Technology, 1997, 126.

34. Wikipedia search: keywords "HCCI", "HCCI Prototypes"

35. Glass, I.I., and Hall, J. Gordon. NAVORD Report 1488, Handbook of Supersonic Aerodynamics Section 18: Shock Tubes. Bureau of Naval Weapons, 1959. 


\title{
Appendix A: Source Code Used
}

\section{Quasi-Steady Main Program and Associated Subroutines}

\author{
MAIN \\ clear all; \\ for $\mathrm{BIGLOOP}=1: 1: 3$ \\ Tmax $=0$; \\ time_Tmax $=0$; \\ $\%$ Input mathematic constants \\ lconv $=0.3048 / 12$; \\ Vconv $=1 \mathrm{e}-6$; \\ $\%$ Input ambient air constants \\ $\mathrm{R}=287$; \\ gamma_amb=1.4; \\ $\mathrm{k}=1.4$; \\ $\mathrm{Cp}=\mathrm{R} * \mathrm{k} /(\mathrm{k}-1)$; \\ $\mathrm{Cv}=\mathrm{R} /(\mathrm{k}-1)$; \\ $\mathrm{pamb}=101325$; \\ $\mathrm{Tamb}=288$; \\ rhoamb $=$ pamb/(R*Tamb); \\ $\%$ Input engine coolant temperature \\ $\mathrm{Tw}=(200+460) *(5 / 9)$; \\ $\%$ Input A cylinder geometries \\ bore_ $\mathrm{a}=(1)^{*} \mathrm{lconv}$; \\ conrod_a $=(2.5) *$ lconv; \\ Vdisp_a $=(30) *$ Vconv; \\ stroke_a $=4 *$ Vdisp_a/ $/$ pi*bore_a^2); \\ $\operatorname{lmax} \mathrm{a}=$ conrod_a+stroke_a/2; \\ $\mathrm{CR} \quad \mathrm{a}=30$; \\ Vtdc_a $=$ Vdisp_a/(CR_a-1); \\ Vbdc_a $=$ Vtdc_a*CR_a; \\ $\%$ Input A-F cylinder geometries \\ bore_af $=(1) *$ lconv; \\ conrod_af $=(2.5) *$ lconv; \\ Vdisp af $=(30) *$ Vconv; \\ stroke_af $=4 *$ Vdisp_af $/\left(\right.$ pi*bore_af $\left.{ }^{\wedge} 2\right)$; \\ $\operatorname{lmax}$ af $=$ conrod af + stroke af $/ \overline{2}$; \\ CR_af $=8$; \\ Vtdc af=Vdisp af/(CR af-1); \\ Vbdc_af $=V t d c \_a f * C R \_a f ;$ \\ $\%$ Set number of cycles and resolution \\ $\mathrm{N}=3$; \\ $\mathrm{dCA}=10$; \\ $\%$ Input engine operating speed \\ RPM $=5400+($ BIGLOOP-1)*600; \\ RPS $=$ RPM $/ 60$; \\ $\mathrm{RPM} \mathrm{ccv}=\mathrm{RPM} / 2$; \\ $\mathrm{w}=\mathrm{RP}$ M_ccv*pi $/ 30$; \\ $\mathrm{Sp} \mathrm{a}=2 *$ stroke $\mathrm{a} * \mathrm{RPM} / 60$; \\ Sp_af $=2 *$ stroke_af*RPM/ 60 ; \\ $\%$ Calculate time increment and create time vector \\ $\mathrm{dt}=1 /(\mathrm{RPS} * \mathrm{dCA} * 360)$; \\ $\mathrm{t}=\left(1: 1: \mathrm{N}^{*} 720 * \mathrm{dCA}\right) * \mathrm{dt}$;
}


$\%$ Set CA limits of operation for valves and max valve areas

n_bdc $1=180 * \mathrm{dCA}$;

n_bdc $2=540 * \mathrm{dCA}$;

n_tdc_ $1=360 * \mathrm{dCA}$;

n_tdc_ $2=720 *$ dCA;

n_inex_open $=15 * \mathrm{dCA}$;

D_i_af $=0.015$;

A_i_af_max $=$ pi*(D_i_af $)^{\wedge} 2 / 4$;

D_i_a $=0.015$

A i a $\max =\mathrm{pi}^{*}(\mathrm{D} \text { i a })^{\wedge} 2 / 4$

D_e_af $=0.015$;

A_e_af_max $=$ pi*(D_e_af) ${ }^{\wedge} 2 / 4$;

D_e_a $=0.015$;

A_e_a_max $=p i *\left(D \_ \text {e_a }\right)^{\wedge} 2 / 4$;

$\%$ Set cylinder volumes as a funtion of CA

for $\mathrm{i}=1: 1: \mathrm{N} * 720 * \mathrm{dCA}$

CA $\operatorname{deg}(\mathrm{i})=(\mathrm{i}-1) / \mathrm{dCA}$;

CA(i) $=\left(C A \_d e g(i) * p i / 180\right)$;

ladj_a $=$ conrod_a*cos(asin $\left.\left(\operatorname{stroke} \_a * \sin (C A(i)) /\left(2 * \operatorname{conrod} \_a\right)\right)\right)$;

h_a(i) $=\operatorname{lmax} \_\mathrm{a}-\left(\mathrm{ladj} \_\mathrm{a}+\right.$ stroke_a* $\left.\cos (\mathrm{CA}(\mathrm{i})) / 2\right)$;

ladj_af $=$ conrod_af*cos(asin(stroke_af*sin(CA(i) $) /(2 *$ conrod_af $))$ );

h_af(i) $=$ lmax_af-(ladj_af+stroke_af*cos(CA(i))/2);

$\overline{V a}(i)=V t d c \_a+h \_a(i) *{ }^{*} i^{*}$ bore $a^{\wedge} 2 / 4$;

$\operatorname{Vaf}(\mathrm{i})=\mathrm{Vtdc}$ af $+\mathrm{h} \_$af(i)*pi*bore_af ${ }^{\wedge} 2 / 4$;

AWalla $(\mathrm{i})=\overline{\mathrm{Va}}(\mathrm{i}) /\left(\bar{p}^{\mathrm{i}}\right.$ *bore_a $)$;

AWallaf(i) $=\operatorname{Vaf}(\mathrm{i}) /($ pi*bore_af $)$;

end

$\%$ Set "af" intake valve area as a function of CA

for $\mathrm{i}=1: 1:$ :n_inex_open

A_i_af(i) $=(\mathrm{i}-1)^{*}$ A_i_af_max $/$ n_inex_open;

end

for $\mathrm{i}=($ n_inex_open +1$): 1:($ n_bdc_1-n_inex_open $)$

$\mathrm{A}$ i $\overline{\text { af }}(\mathrm{i})=\overline{\mathrm{A}} \mathrm{i}$ af max;

end

for $\mathrm{i}=(\mathrm{n}$ bdc $1-\mathrm{n}$ inex open +1$): 1: \mathrm{n}$ bdc 1

A_i_af(i) $=($ n_bdc_1-i)*A_i_af_max/n_inex_open;

end

for $\mathrm{i}=\left(\mathrm{n} \_\right.$bdc_1+1):1:N*720*dCA

A_i aff(i) $=0$;

end

$\%$ Set "a" intake valve area as a function of CA

for $\mathrm{i}=1: 1$ :n_inex_open

A_i a $(i)=(i-1)^{*}$ A_i_a_max/n_inex_open;

end

for $\mathrm{i}=\left(\mathrm{n} \_\right.$inex_open +1$): 1:\left(n \_b d c \_1-n \_i n e x \_o p e n\right)$

A_i_a $(i)=\bar{A} \_$i_a_max;

end

for $\mathrm{i}=\left(\mathrm{n} \_\right.$bdc_1-n_inex_open +1$): 1: \mathrm{n} \_$bdc 1

A i $\left.\bar{a}(\mathrm{i})=\overline{(n} \_\overline{d c} \_1-\bar{i}\right)^{*} \mathrm{~A}$ i a max $/ n \_$inex_open;

end

for $\mathrm{i}=(\mathrm{n}$ bdc $1+1): 1: \mathrm{N} * 720 * \mathrm{dCA}$

A_i_a $(i)=0$;

end

$\%$ Set "af" exhaust valve area as a function of CA

for $\mathrm{i}=1: 1: \mathrm{n} \_$bdc_2

A_e_af(i) $=0$;

end

for $\mathrm{i}=\left(\mathrm{n} \_\right.$bdc $\left.\_2+1\right): 1:\left(\mathrm{n} \_\right.$bdc $\_2+1+\mathrm{n} \_$inex_open $)$

A_e_af(i) $=\left(i-\left(n \_b d c \_2+1\right)\right) * A \_e \_a f \_m a x / n \_i n e x \_o p e n ;$

end

for $\mathrm{i}=\left(\mathrm{n} \_\right.$bdc $\_2+2+n \_$inex_open $): 1:\left(n \_t d c \_2-n \_\right.$inex_open $)$

A_e_af(i)=A_e_af_max;

end

for $\mathrm{i}=\left(\mathrm{n} \_t d c \_2-n \_\right.$inex_open +1$): 1: \mathrm{n} \_$tdc 2

A e $\operatorname{af}(\mathrm{i})=(\mathrm{n}$ tdc $2-\mathrm{i}) * \mathrm{~A}$ e af $\max / \mathrm{n}$ inex open;

end 


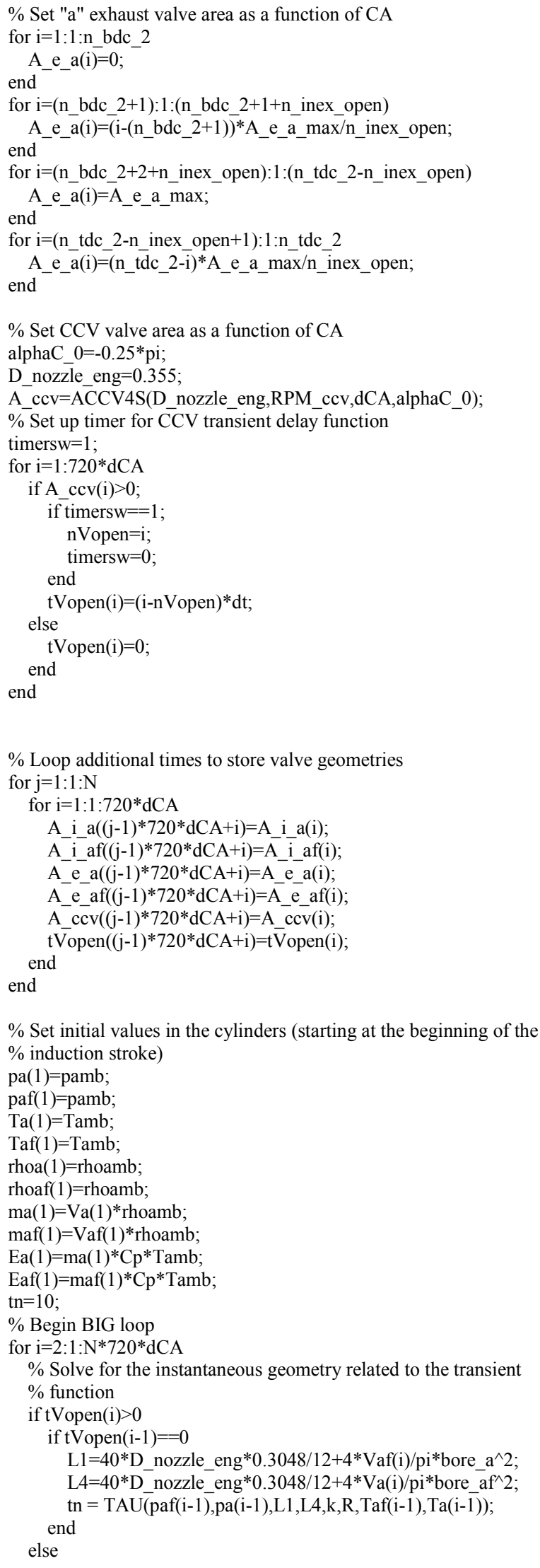




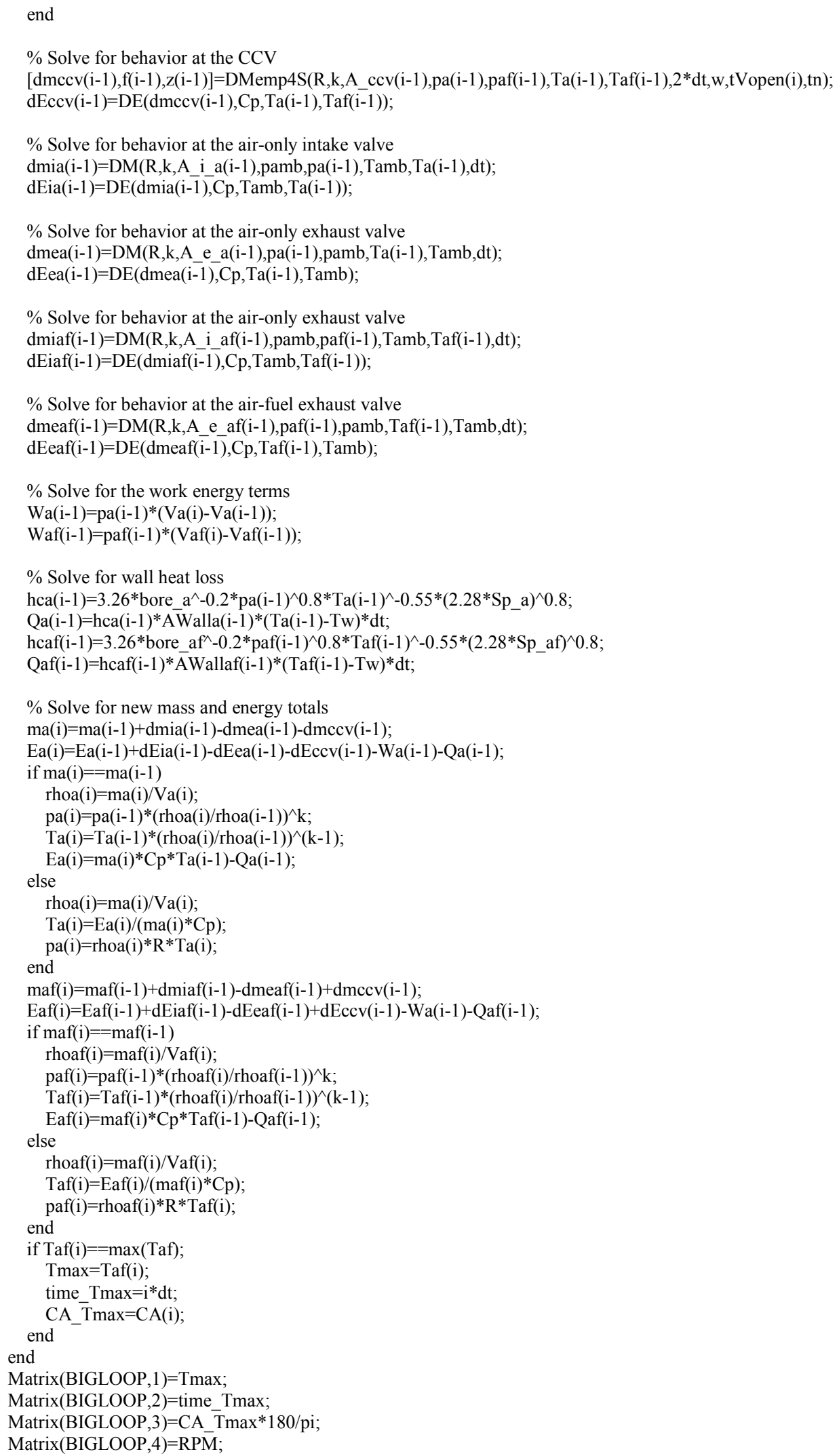




\section{MODIFIED MASS FLOW RATE}

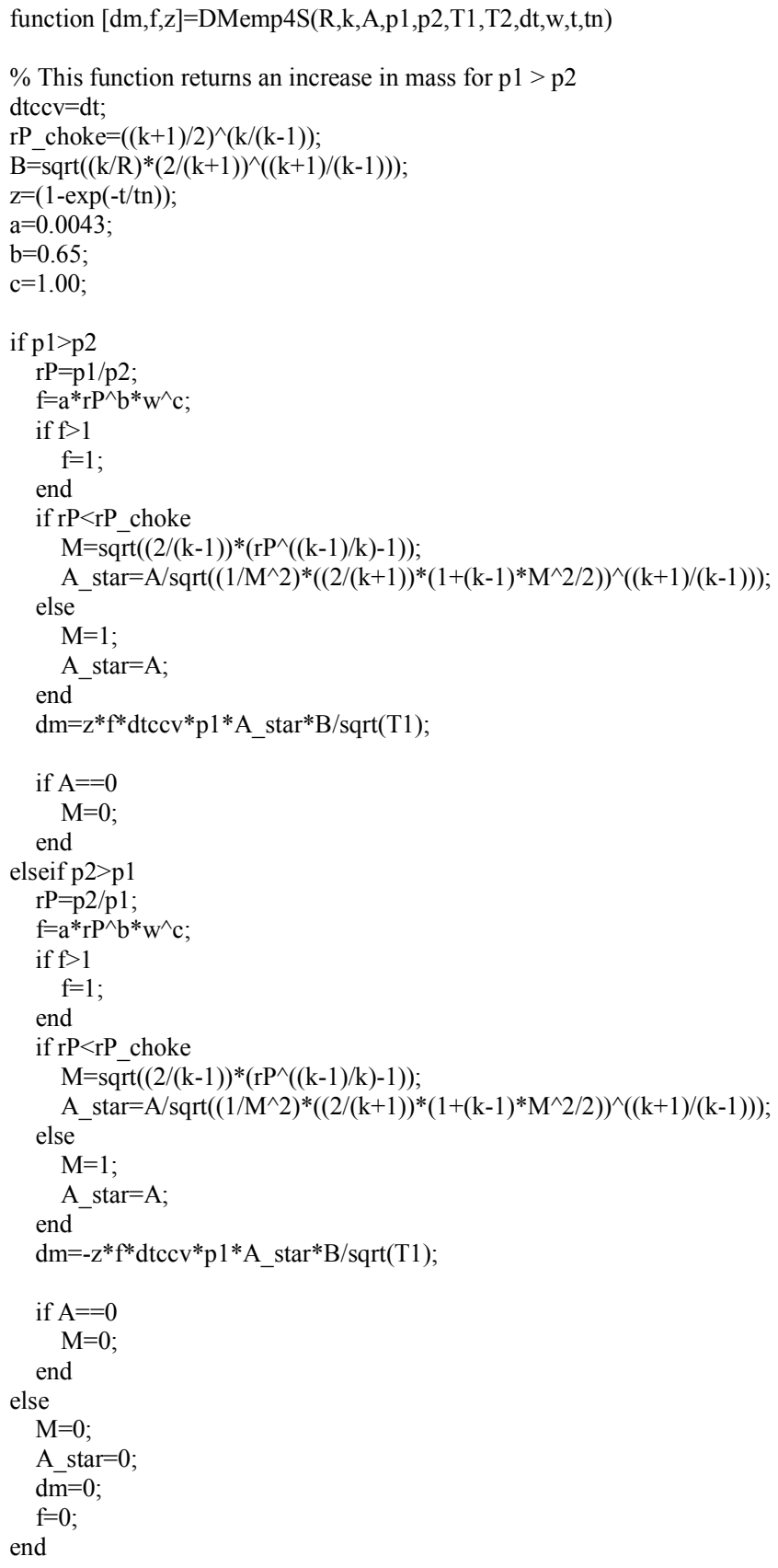




\section{ENERGY FLOW RATE}

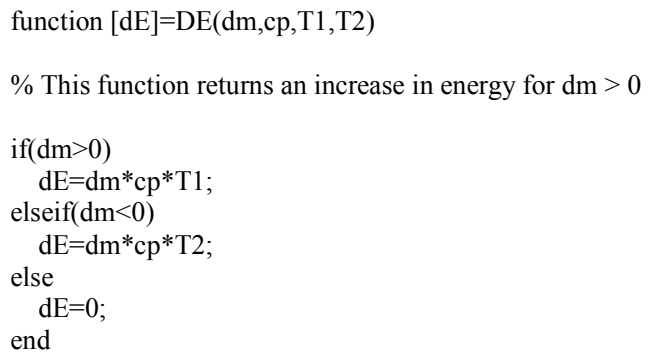

\section{TRANSIENT TIME CONSTANT}

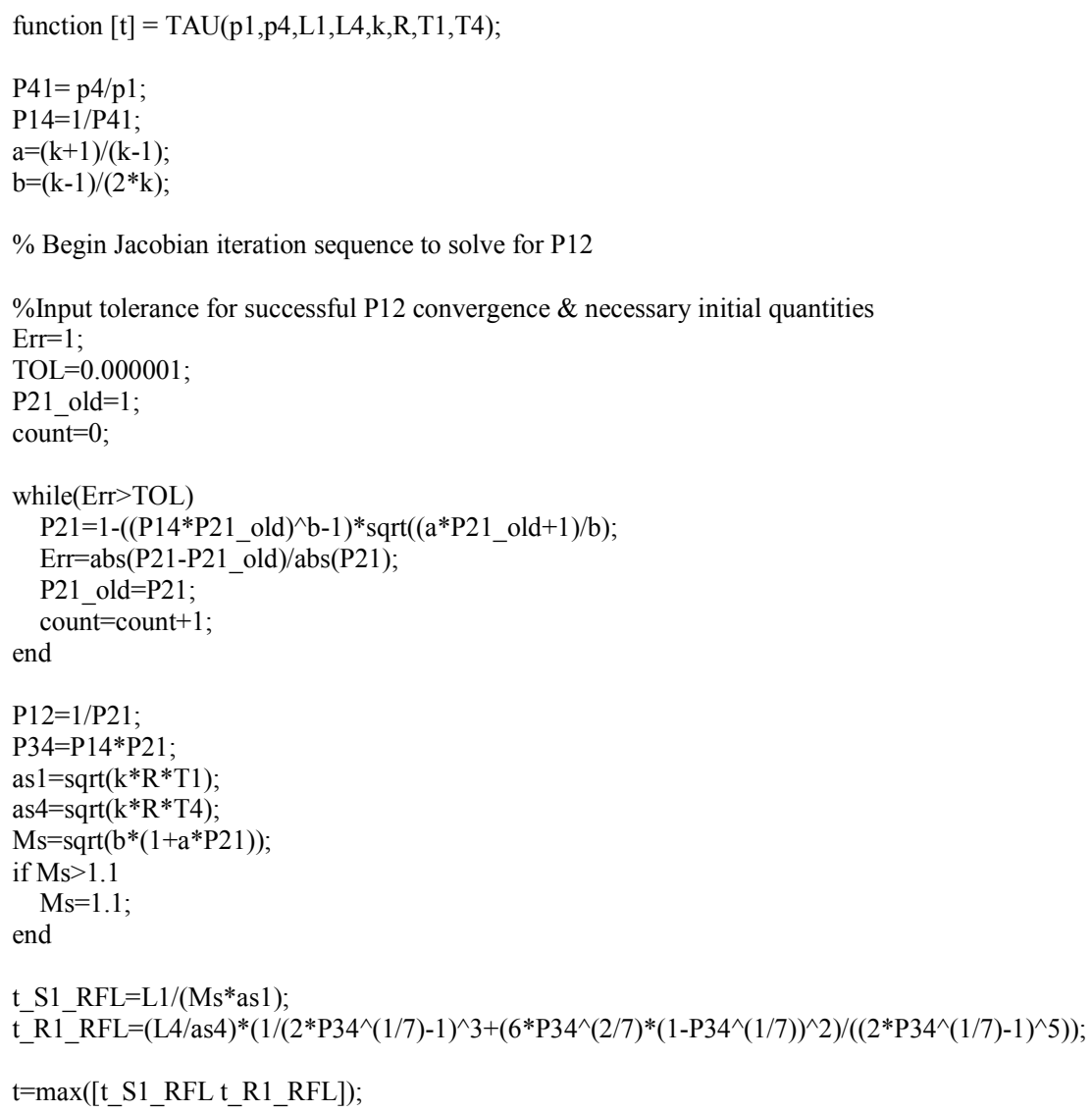




\section{AREA EVOLUTION}

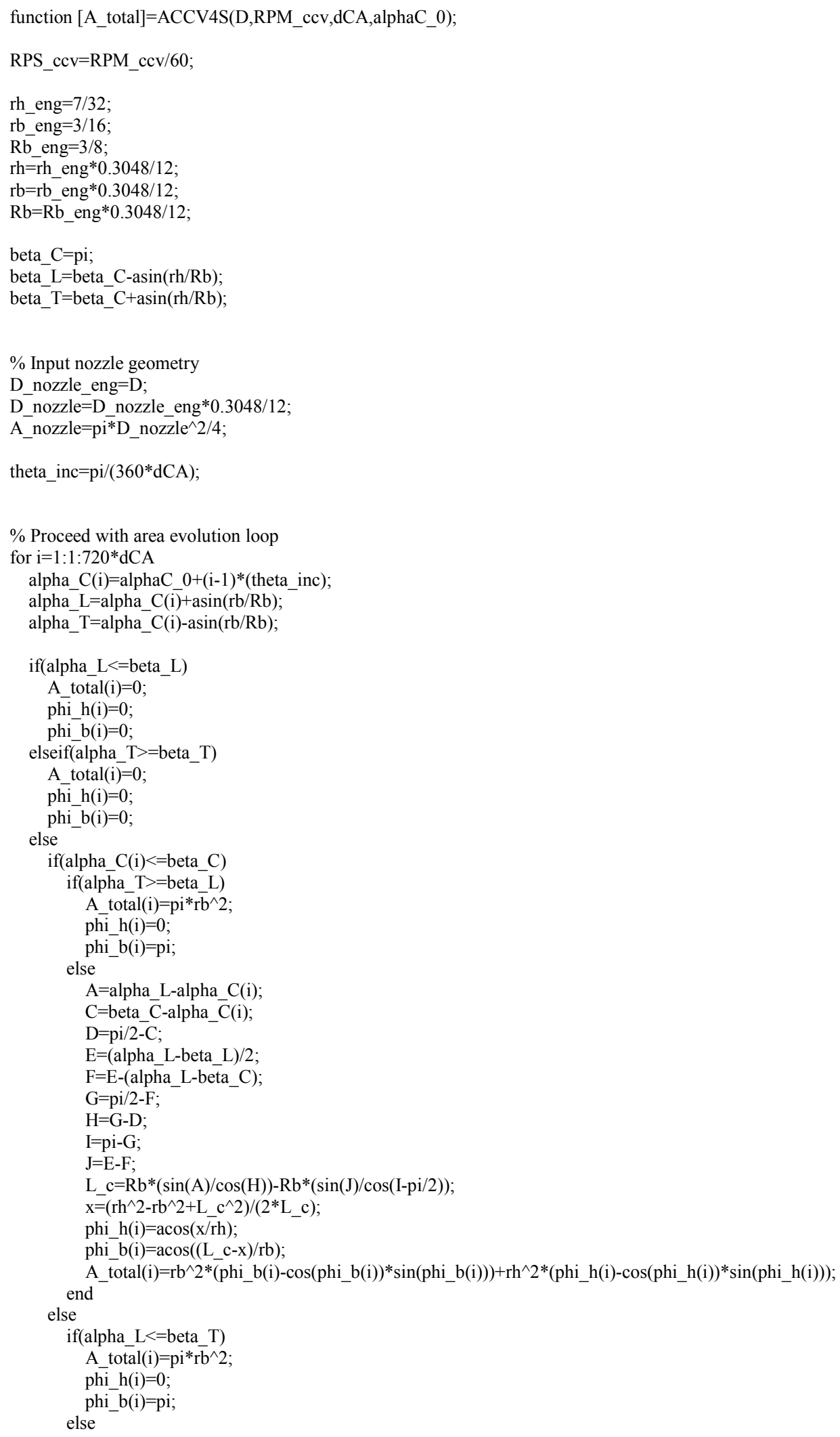




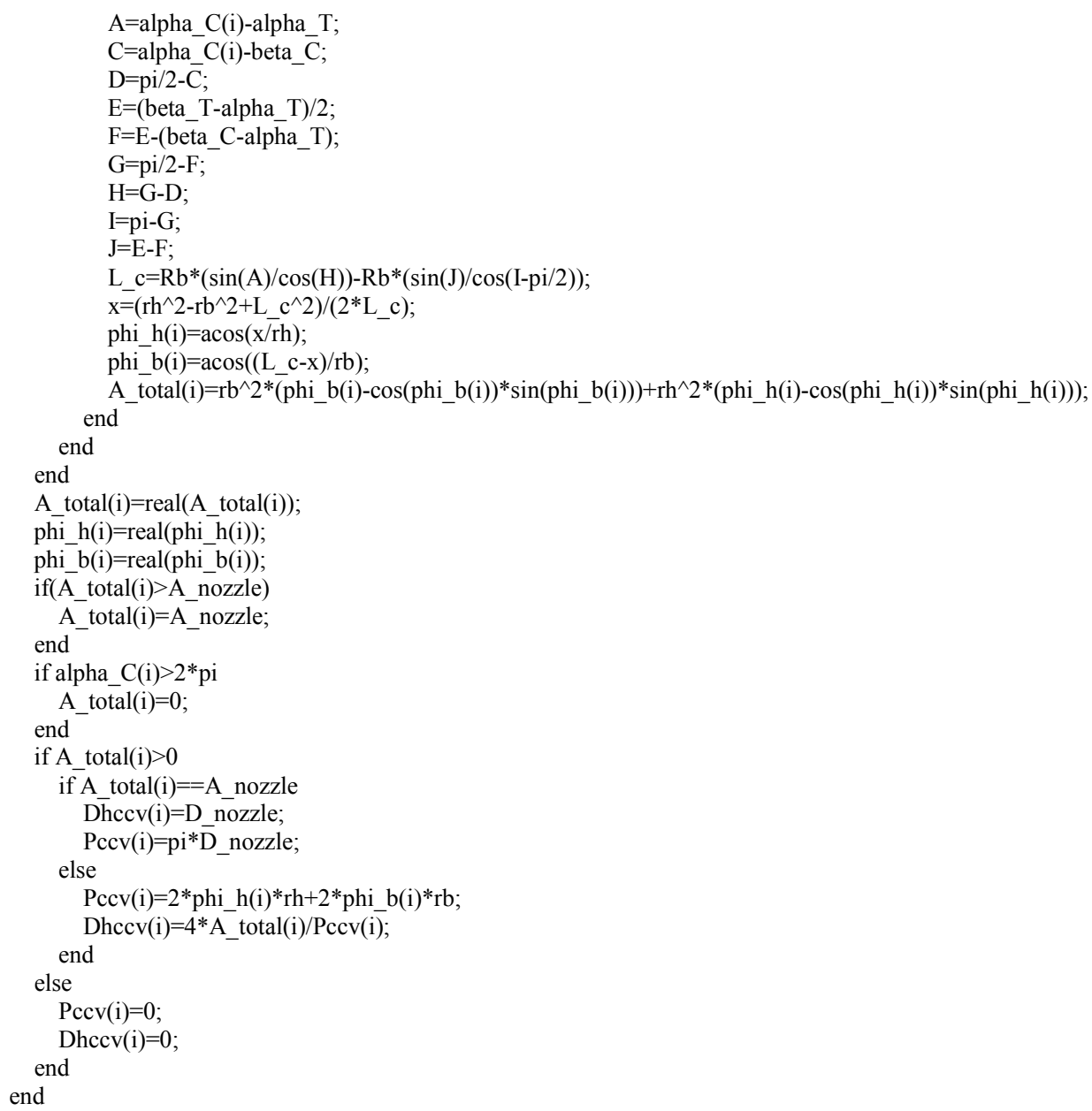




\section{Omega DAQP-208H C++ Data Acquisition Control Code \\ The following code was written for use with Microsoft Visual $\mathrm{C}++$ to control data acquisition through the Omega DAQP-208H PCMCIA data acquisition system.}

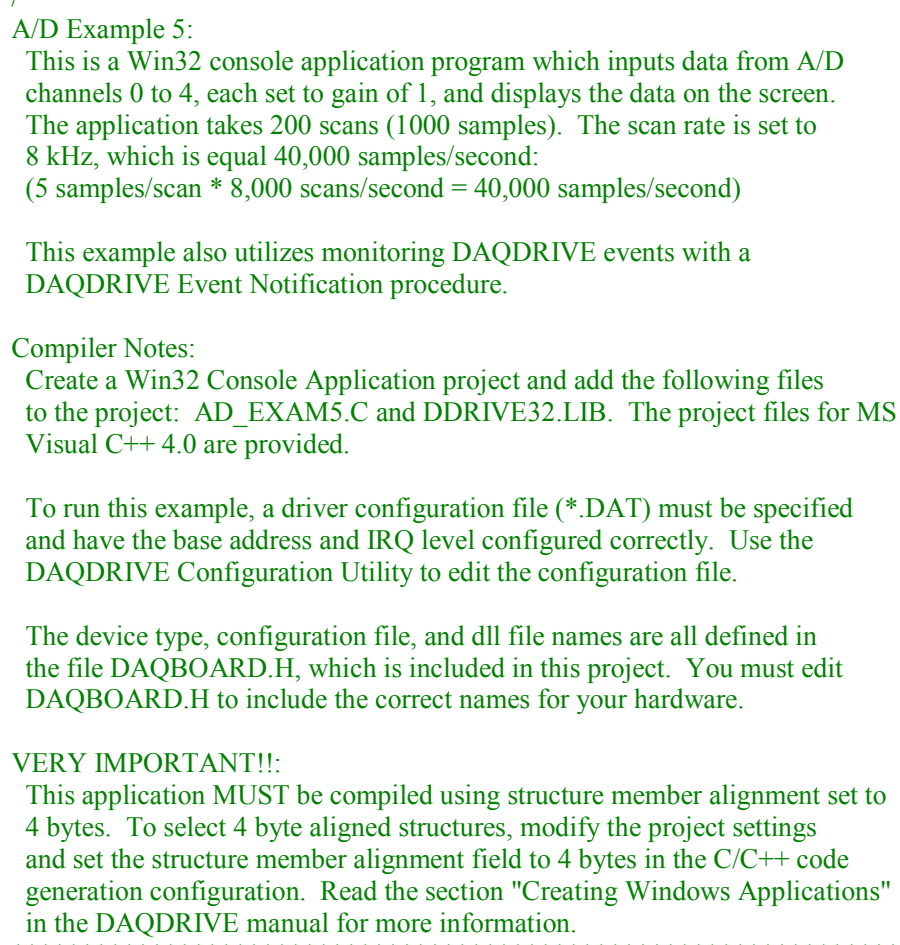


unsigned short main(void)

\{

FILE * pFile;

// All memory accessed by DAQDRIVE during interrupt service routines must be //

// allocated by DAQAllocateRequest, which dynamically allocates and locks the //

// memory for the DAQDRIVE structures and variables.

//

// Create null pointers for the data structures returned by DaqAllocateRequest

struct DAQDRIVE_buffer *my_data; // null pointers for daqdrive_buffer structure

struct ADC_request *user_request; // null pointer for user_request structure

signed short *input_data; // null pointer to short for user buffers

unsigned short *channel_array; // null pointer for A/D channel array

float $\quad$ *gain_array; // null pointer for A/D gain array

// Structures and variables not used within the DAQDRIVE interrupt service routine

struct allocate_request memory_request; // used to dynamically allocate structures and buffers

struct sigcon_request conv_request; // structure used to convert results to "real world" units

double $\quad *$ convert_data;

unsigned short $\quad$ logical_device $=0$; // DAQDRIVE device ID

unsigned short request_handle $=0$; // DAQDRIVE device handle

unsigned short status; // DAQDRIVE status

unsigned short

unsigned long

$\mathrm{i}, \mathrm{j}$;

event mask;

float daqdrive_version; // DAQDRIVE version

float software_version; // hardware driver version

float firmware version; // hardware firmware version

// har kb_char;

unsigned short output_channel;

short output_value;

//short DA_square[scans];

memory_request.memory_handle $=0$; // initialize for error handling procedure

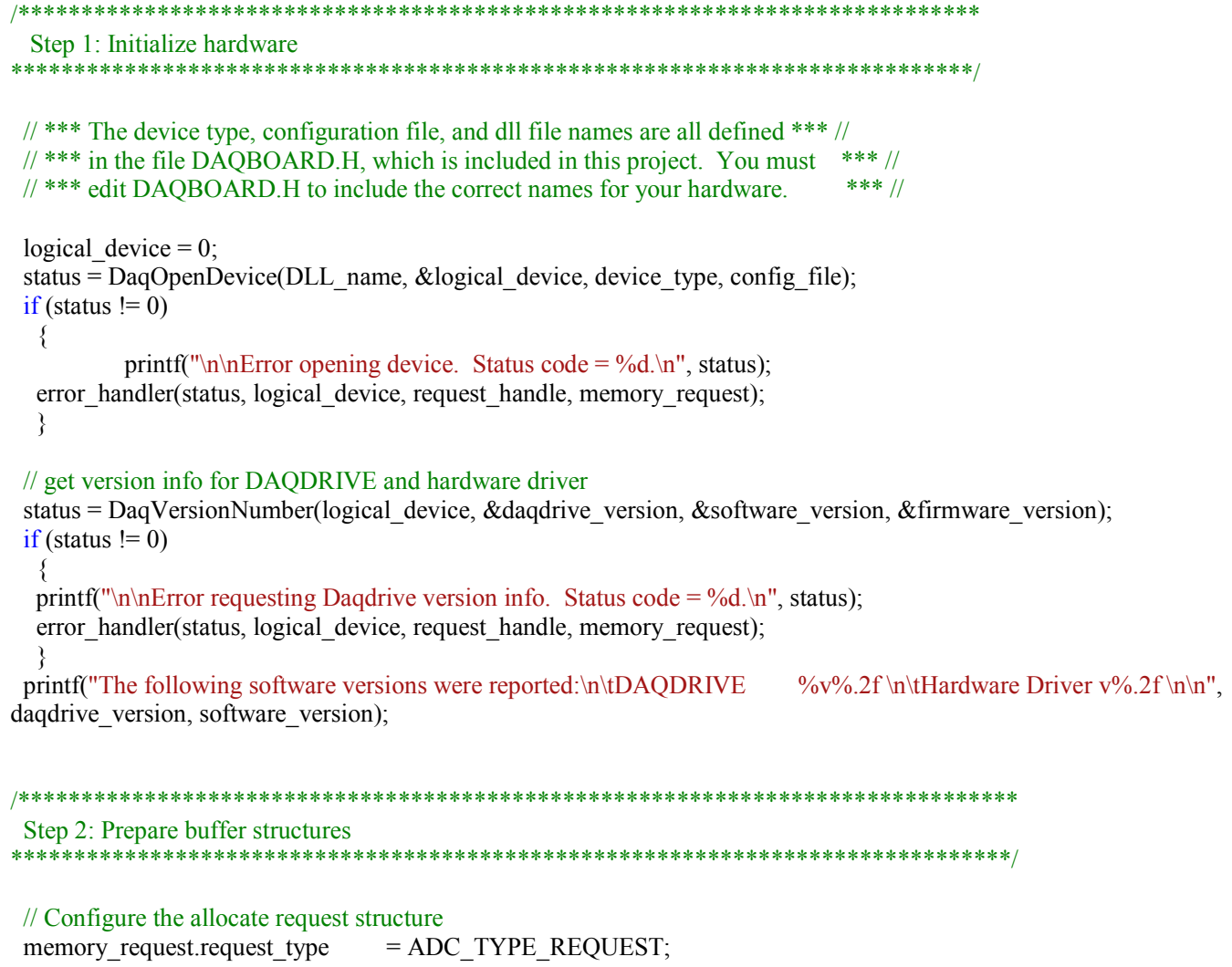




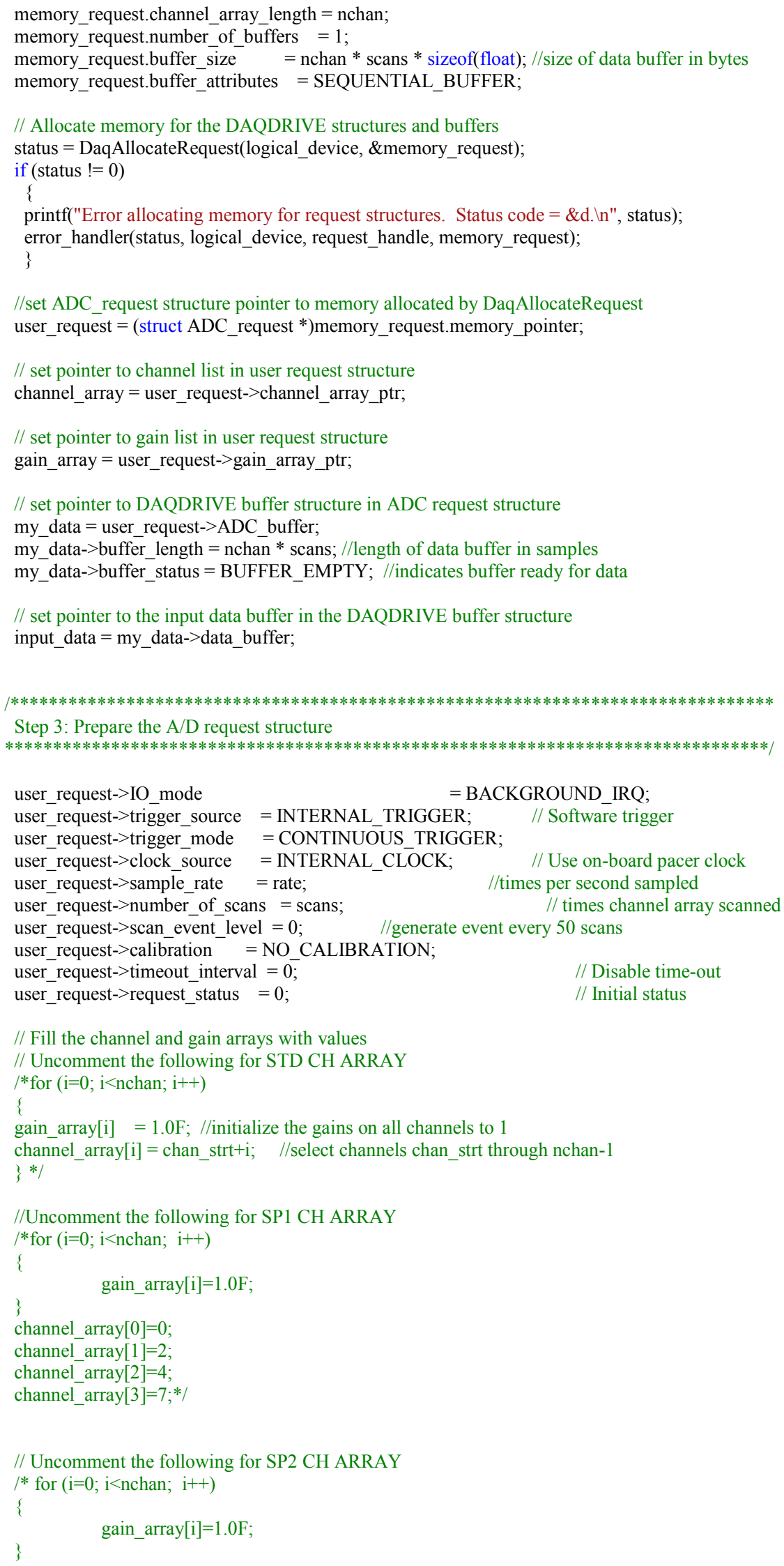




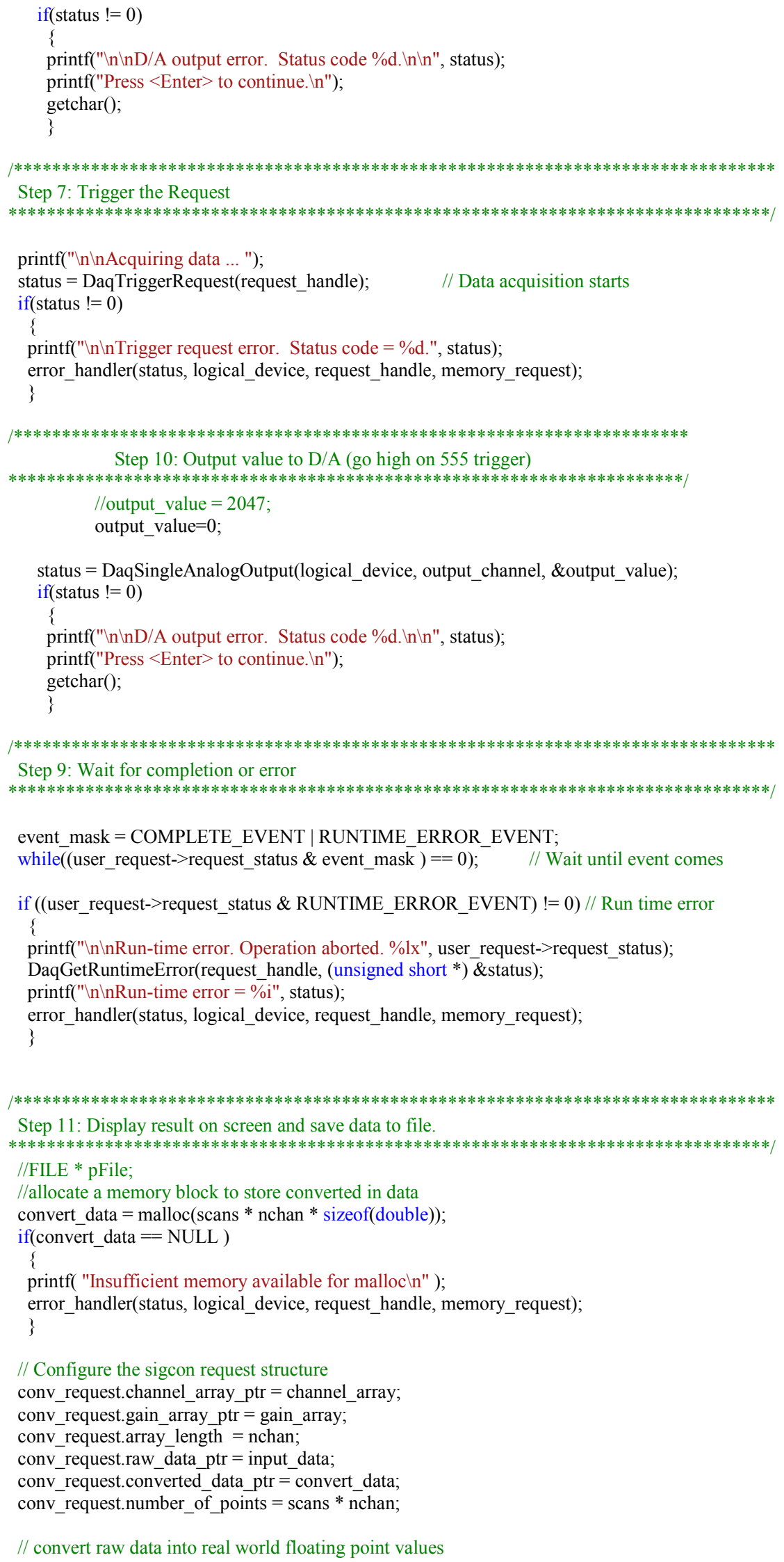




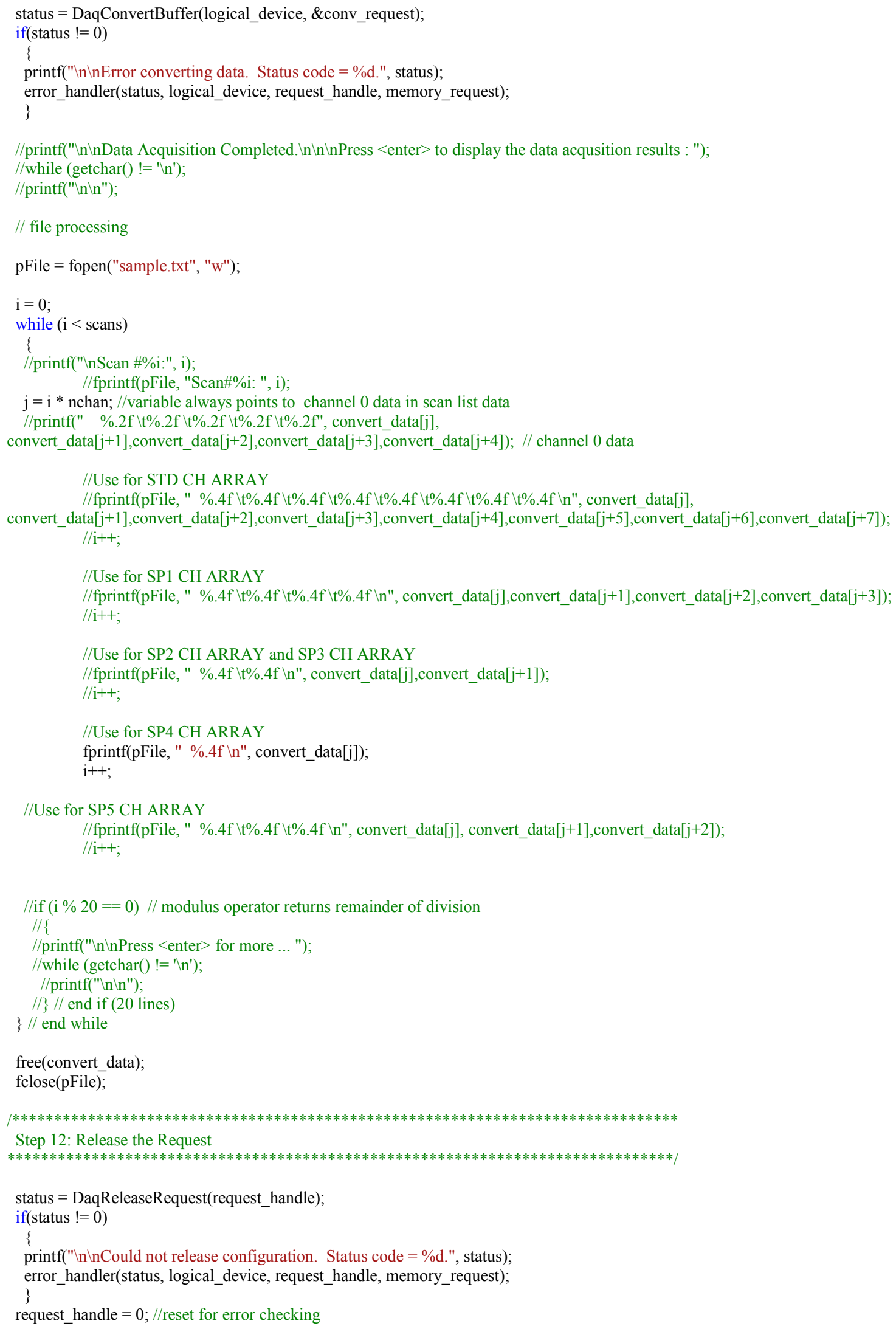




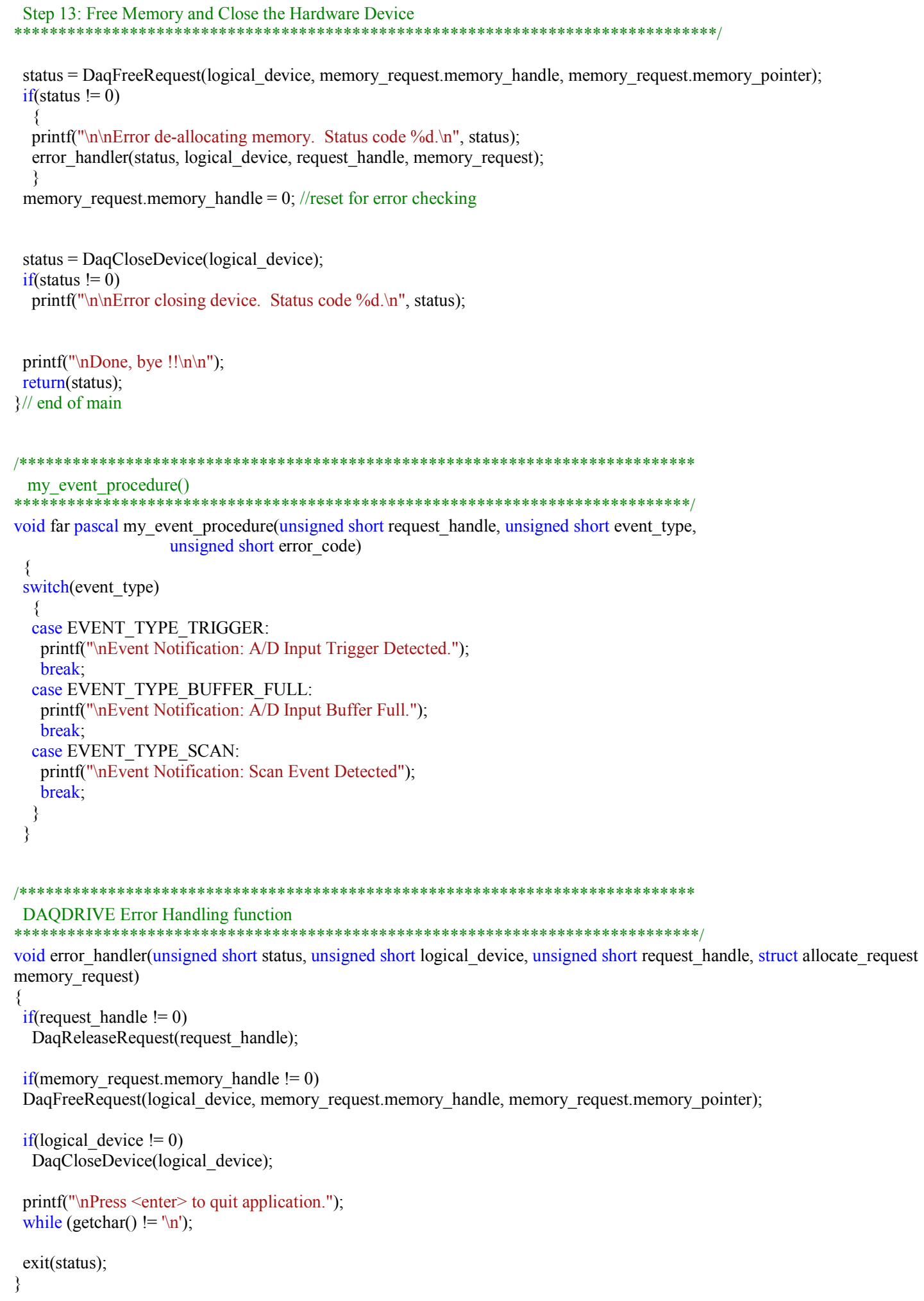




\section{Appendix B: Experimental Sensor Calibration}

Two sensors needed to be calibrated to the particular environment and intended use. The tank pressure transducer (PX-105) was calibrated versus a known secondary digital pressure gauge over the range of pressures intended for the experiments. The multipoint calibration curve and the resulting trend line fit are shown in Figure 68. The equation used to determine the tank pressure was found as

$$
p_{\text {TANK }}=\left(96.949 V_{\text {out }}-86.661\right) \text { psig }
$$

or

$$
p_{\text {TANK }}=\left(96.949 V_{\text {out }}-86.661+p_{\text {amb }}\right) p s i a
$$

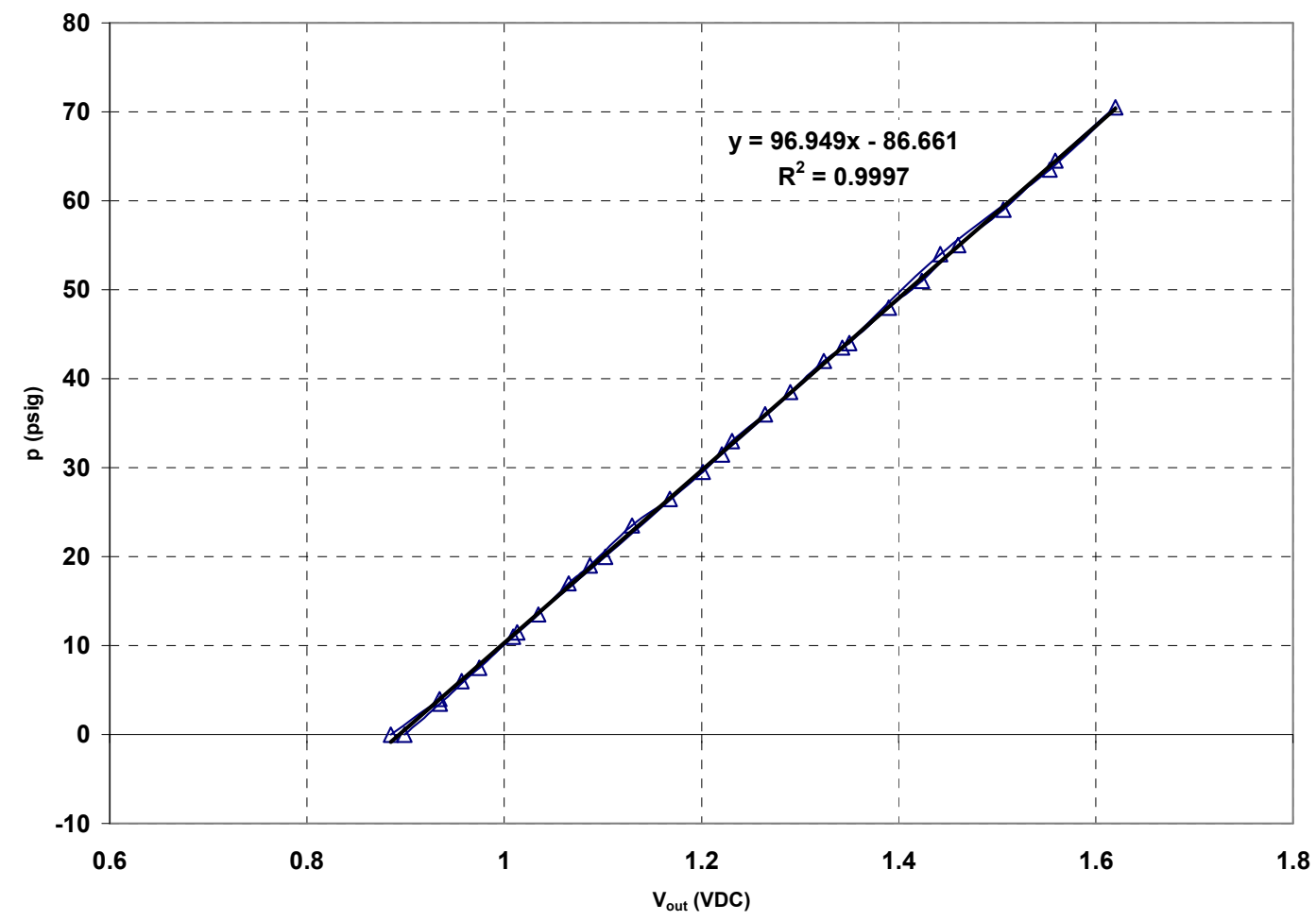

Figure 68: The calibrated curve of the PX-105 pressure transducer used in the second stage tank. 
The thermocouple used to determine tank temperature was calibrated using a simple 2 point calibration method. The two points used were an insulated ice bath and a pressure compensated water boiling point. Figure 69 show the calibrated curve, which demonstrates the nearly ideal 1000 to 1 relationship between the millivolt response voltage and the sample temperature.

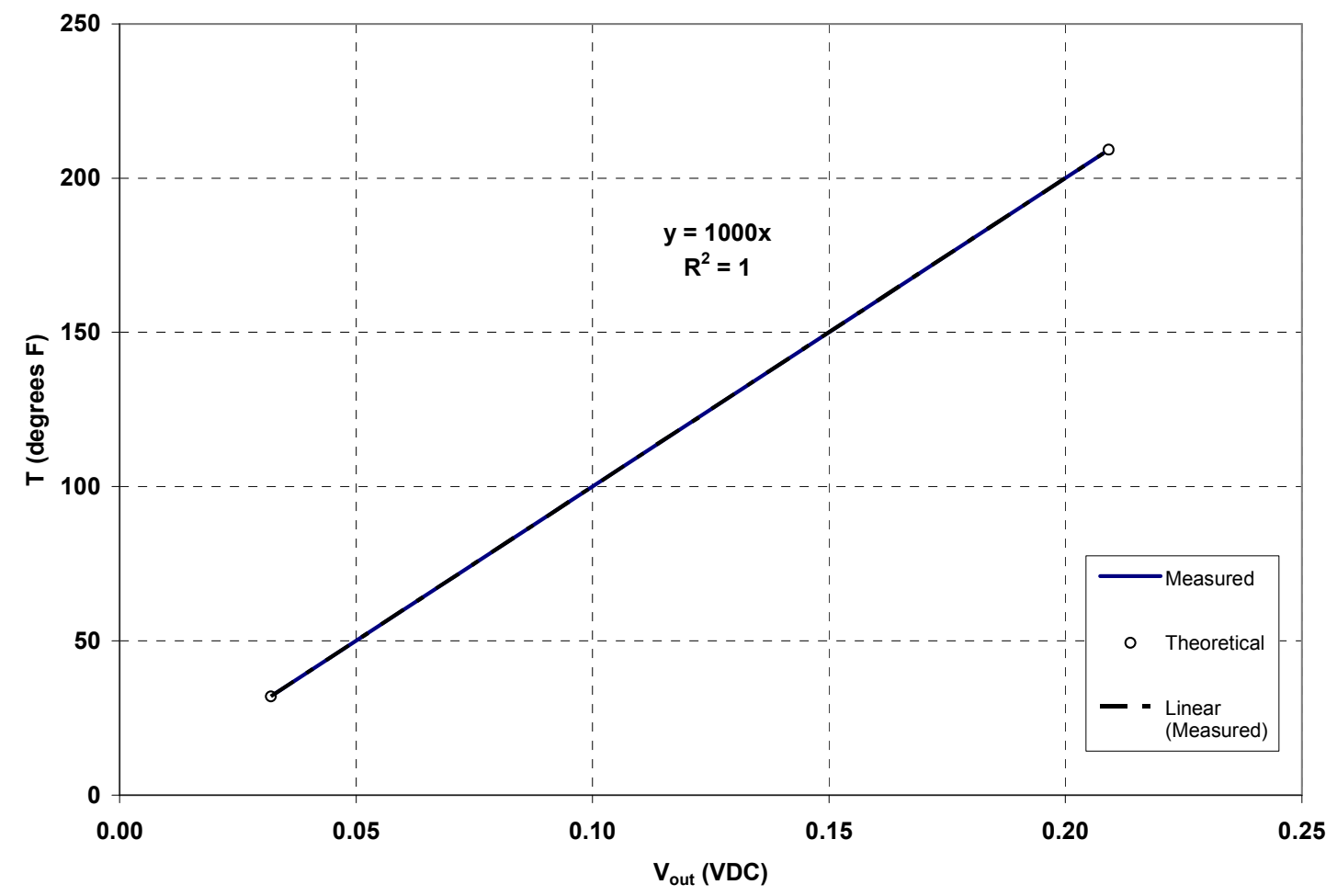

Figure 69: The calibration curve of the tank thermocouple. 


\section{Appendix C: Error Analysis}

\section{Error in calculated injected mass:}

To determine the mass of injected air from a known final collection tank pressure in inches $\mathrm{H}_{2} 0$,

$$
\begin{aligned}
& m_{I N J}=m_{f}-m_{i}=\left(\frac{p_{f}}{R T}\right)\left(h_{c} A_{c}+\forall_{\text {tube }}\right)-\left(\frac{p_{\text {amb }}}{R T}\right)\left(h_{i} A_{c}+\forall_{\text {tube }}\right) ; \\
& m_{I N J}=\left[\frac{p_{\text {amb }}+\rho_{H_{2} O} g\left(h_{c}-h_{r}\right)}{R T}\right]\left(h_{c} A_{c}+V_{\text {tube }}\right)-\left(\frac{p_{\text {amb }}}{R T}\right)\left(h_{i} A_{c}+\forall_{\text {tube }}\right)
\end{aligned}
$$

where $\left(h_{c}-h_{r}\right)$ is known and

$$
\begin{aligned}
& h_{c}=\frac{\left(h_{c}-h_{r}\right)}{D}+h_{i} \\
& D=\left(1+\frac{A_{c}}{A_{r}}\right)
\end{aligned}
$$

Beginning with the measurement for ambient pressure, the WS-110 was capable of reading accurately to within $\pm 1500 \mathrm{~Pa}$, thus for a typical sample,

$$
\begin{aligned}
& p_{\text {amb }}=(95,000 \pm 1500) P a \\
& e r r_{p_{a m b}}= \pm 1.58 \%
\end{aligned}
$$

The WS-110 was capable of reading the ambient temperature accurately to within $\pm 1 \mathrm{~K}$, thus for a typical sample,

$$
\begin{aligned}
& T_{a m b}=(299 \pm 1) K \\
& e r r_{T_{a m b}}= \pm 0.334 \%
\end{aligned}
$$


The water levels for initial and final height readings were measured using a steel rule marked in 64ths of an inch, thus for any measurement the error was limited to $\pm(1 / 128)$ "'. Relatively large length measurements required for calculating the surface area of the reservoir were taken using a rule marked in 16ths of an inch while relatively short lengths were measured using a Vernier caliper marked in 0.002 ", thus for the error in the area,

$$
A_{R}=\left(13 \times 4-\frac{\pi O D^{2}}{4}\right)=(13 \pm 1 / 32)(4 \pm 1 / 32)-\frac{\pi}{4}(3.52 \pm .001)^{2}
$$

The maximum likely error may be found for the reservoir area as

$$
\begin{aligned}
& e r r_{A_{R}}=\left(0.788^{2}+0.0402^{2}\right)^{1 / 2} \\
& e r r_{A_{R}}= \pm 0.789 \%
\end{aligned}
$$

The error for the collection chamber volume is similarly found as

$\operatorname{err}_{A_{C}}= \pm 0.0468 \%$

The syringes used to determine many of the various volumes throughout the experimental apparatus were marked in milliliters, thus for the particular volume measurement,

$$
\begin{aligned}
& V_{\text {tube }}=(125 \pm 0.5) c c \\
& e r r_{V_{\text {tube }}}= \pm 0.400 \%
\end{aligned}
$$

Assembling all the relevant terms, the error for a typical single injection mass calculation was found as

$$
e r r_{m_{i n j}}= \pm 2.972 \%
$$

\section{Error in calculated shock Mach number}

To determine the Mach number of the passing fluid in the shock tube, 


$$
M_{S}=\frac{\left(\frac{x_{2}-x_{1}}{t_{2}-t_{1}}\right)}{\sqrt{\gamma R T_{a m b}}}
$$

Beginning with the error in locations for the microphones, the lengths were measured using the caliper such that the error was found as

$\left(x_{2}-x_{1}\right)=(10.500 \pm 0.001) "$

$e r r_{\Delta x}= \pm 0.0095 \%$

The digitized time signals were only as certain as half the sampling rate, or

$\left(\frac{n_{1}}{f_{s}} \pm \frac{1}{2 f_{s}}\right)-\left(\frac{n_{2}}{f_{s}} \pm \frac{1}{2 f_{s}}\right)$

A typical near sonic sample taken using a sampling frequency of $50 \mathrm{kHz}$ has a time error calculated as

$e r r_{\Delta t}= \pm 3.72 \%$

The maximum likely error in the calculation for the ambient speed of sound is

$$
e r r_{a_{a m b}}= \pm 0.166 \%
$$

Taking into account all sources of measurement error, the maximum likely error of the Mach number calculations is found as

$$
\begin{aligned}
& e r r_{M_{S}}=\left(0.0095^{2}+3.72^{2}+0.166^{2}\right)^{1 / 2} \\
& e r r_{M_{S}}= \pm 3.72 \%
\end{aligned}
$$

\section{Error in calculated pressure ratio}

The pressure ratio was calculated according to the following formula 


$$
r_{p}=\frac{p_{T A N K}}{p_{a m b}}
$$

The PX-105 was capable of accurately measuring the internal pressure of the tank to within $\pm 1724 \mathrm{~Pa}$, thus for a low tank pressure (which would be the worst case error),

$p_{\text {TANK }}=(239,182 \pm 1724) P a$

err $r_{p_{\text {TANK }}}= \pm 0.721 \%$

Incorporating the previously determined typical ambient pressure error, the maximum likely error for the pressure ratio measurement is found to be

$e r r_{r_{p}}=\left(0.721^{2}+1.58^{2}\right)^{1 / 2}$
$e r r_{r_{p}}= \pm 1.74 \%$

\section{Error in CCV shaft speed calculations}

The shaft speed calculations used for correlations involving the constant volume experiments were made according to the following formula

$$
R P M=\frac{N}{\Delta t}
$$

where $\mathrm{N}$ and $\Delta \mathrm{t}$ were determined using a Jagabi hand tachoscope. Random error analysis was performed for all 5 speed settings of the CCV motor and errors were determined over the $95 \%$ confidence interval:

Speed \#1:

$\sigma=5.912, \quad \alpha=3.413$

speed $\# 1=(933.8 \pm 15.000) R P M$

$e r r_{\text {spd\#1 }}= \pm 1.606 \%$ 
Speed \#2:

$\sigma=3.338, \quad \alpha=1.927$

speed $\# 2=(994.7 \pm 8.469) R P M$

$e r r_{\text {spd \#2 }}= \pm 0.8514 \%$

Speed \#3:

$\sigma=5.679, \quad \alpha=3.279$

speed $\# 3=(985.3 \pm 14.41) R P M$

$e r r_{\text {spd\#3 }}= \pm 1.463 \%$

Speed \#4:

$\sigma=3.149, \quad \alpha=1.818$

speed $\# 4=(1034.5 \pm 7.99) R P M$

$e r r_{\text {spd\# } 4}= \pm 0.7724 \%$

Speed \#5:

$\sigma=6.930, \quad \alpha=4.000$

speed $\# 5=(1112.1 \pm 17.6) R P M$

$e r r_{\text {spd } \# 5}= \pm 1.581 \%$ 


\section{Vita}

\section{Patrick H Browning}

\section{Education and Experience}

Patrick Browning received the degree of Bachelor of Science in Aerospace Engineering Summa Cum Laude from the College of Engineering and Mineral Resources of West Virginia University in 2005 (3.95 GPA). One of the first students to be accepted into the college's fast track option, he subsequently received the degree of Doctor of Philosophy in Aerospace Engineering from the same college in 2009 (3.92 GPA). His coursework prepared him specifically for the areas of aerodynamics and thermodynamics, and his written doctoral examination reflected those chosen topics. During his years at the CEMR, he worked as a graduate research assistant for Dr. John Loth in a variety of different aerospace related research areas including:

- natural gas pipeline acoustics and infringement detection;

- unmanned aerial vehicle (UAV) design and fabrication;

- performance analysis of conventional aircraft and dual rotor helicopters for military UAV's

- gas ejector systems;

- wingtip circulation control (CC) and boundary layer control (BLC);

- air-injected compression ignition in a Cooperative Fuel Research (CFR) engine;

- hot dip steel galvanizing air knife fabrication and control.

Patrick Browning also worked as a teaching assistant for several courses, including:

- MAE 215 Introduction to Aerospace Engineering

- MAE 336 Compressible Aerodynamics

- MAE 426 Jet Propulsion

- MAE 322 Thermal and Fluids Laboratory

\section{Objectives}

Browning's current plans are to remain in academia as a teaching or research professor in the field of aerospace or mechanical engineering. He has a high level of interest in creating and maintaining a higher degree of intellectual and ethical standards within the general field of engineering, particularly with new college level engineering students.

\section{Personal Interests}

Patrick's main interests involve a high priority on family values and a God-centered life. He has a deep sense of personal responsibility to his family as well as his local community. Personal interests include many different outdoor activities including road and mountain bike riding, backpacking, and lake and river kayaking.

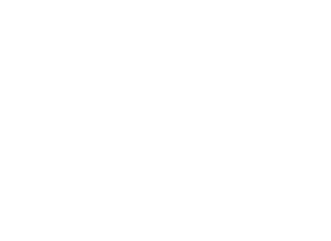

